DOEEEIA-MO69(95)

\title{
Model Documentation
}

\section{Renewable Fuels Module}

\section{of the National Energy Modeling System}

June 1995 -

Prepared by:

Office of Integrated Analysis and Forecasting Energy Supply and Converşion Division Energy Information Administration 1000 Independence Avenue, S.W. Washington, DC 20585

AH 


\section{DISCLAIMER}

This report was prepared as an account of work sponsored by an agency of the United States Government. Neither the United States Government nor any agency thereof, nor any of their employees, make any warranty, express or implied, or assumes any legal liability or responsibility for the accuracy, completeness, or usefulness of any information, apparatus, product, or process disclosed, or represents that its use would not infringe privately owned rights. Reference herein to any specific commercial product, process, or service by trade name, trademark, manufacturer, or otherwise does not necessarily constitute or imply its endorsement, recommendation, or favoring by the United States Government or any agency thereof. The views and opinions of authors expressed herein do not necessarily state or reflect those of the United States Government or any agency thereof. 


\section{DISCLAIMER}

Portions of this document may be illegible in electronic image products. Images are produced from the best available original document. 


\title{
Model Documentation
}

\section{Renewable Fuels Module}

\section{of the National Energy Modeling System}

\author{
June 1995
}

Prepared by:

Office of Integrated Analysis and Forecasting

Energy Supply and Converșion Division

Energy Information Administration

- 1000 Independence Avenue, S.W.

Washington, DC 20585 
Released for Printing: July 20, 1995 


\section{Contents}

1. Renewable Fuels Module Introduction $\ldots \ldots \ldots \ldots \ldots \ldots \ldots \ldots \ldots \ldots \ldots$

Purpose of This Report . . . . . . . . . . . . . . . . . . . 1

Renewable Fuels Module Summary $\ldots \ldots \ldots \ldots \ldots \ldots \ldots \ldots \ldots \ldots$

Municipal Solid Waste Submodule (MSW) $\ldots \ldots \ldots \ldots \ldots \ldots \ldots \ldots, 3$

Wind Energy Submodule (WES) $\ldots \ldots \ldots \ldots \ldots \ldots \ldots \ldots \ldots \ldots \ldots$

Solar Submodule (SOLES) . . . . . . . . . . . . . . . . 3

Biofuels Supply Submodule (BSS) $\ldots \ldots \ldots \ldots \ldots \ldots \ldots \ldots \ldots, 4$

Wood Submodule $\ldots \ldots \ldots \ldots \ldots \ldots \ldots \ldots \ldots \ldots \ldots \ldots \ldots$

Geothermal Electricity Submodule (GES) $\ldots \ldots \ldots \ldots \ldots \ldots \ldots \ldots \ldots 4$

Hydroelectric Plant Data . . . . . . . . . . . . . . . . . . .

Archival Media $\ldots \ldots \ldots \ldots \ldots \ldots \ldots \ldots \ldots \ldots \ldots \ldots \ldots$

Model Contact $\ldots \ldots \ldots \ldots \ldots \ldots \ldots \ldots \ldots \ldots \ldots \ldots \ldots \ldots$

Report Organization $\ldots \ldots \ldots \ldots \ldots \ldots \ldots \ldots \ldots \ldots \ldots \ldots \ldots \ldots 7$

2. Municipal Solid Waste (MSW) Submodule . . . . . . . . . . . . . . 9

Model Purpose . .......................... 9

Relationship of the MSW Submodule to Other Models ............ 9

Modeling Rationale . ........................... 10

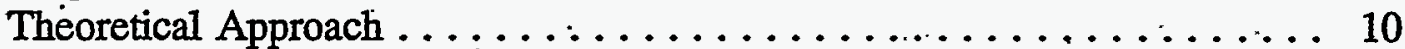

Fundamental Assumptions . . . . . . . . . . . . . . . . . 10

MSW Quantity Projections . . . . . . . . . . . . . . . . 10

Projected Btu Value of MSW . . . . . . . . . . . . . . . 11

Projected Percentage of MSW Combusted With Heat Recovery . . . . . . 11

Disaggregation Rules . . . . . . . . . . . . . . . . . . 12

Capital and Operating Costs $\ldots \ldots \ldots \ldots \ldots \ldots \ldots \ldots \ldots \ldots \ldots \ldots \ldots$

Alternative Approaches..$\ldots \ldots \ldots \ldots \ldots \ldots \ldots \ldots \ldots \ldots \ldots \ldots \ldots \ldots \ldots$

MSW Submodule Structure $\ldots \ldots \ldots \ldots \ldots \ldots \ldots \ldots \ldots \ldots \ldots \ldots 13$

Submodule Flow Diagram $\ldots \ldots \ldots \ldots \ldots \ldots \ldots \ldots \ldots \ldots \ldots$

Key Computations and Equations $\ldots \ldots \ldots \ldots \ldots \ldots \ldots \ldots \ldots \ldots$

Appendices

2-A:- Inventory of Variables, Data, and Parameters $\ldots \ldots \ldots \ldots \ldots \ldots \ldots \ldots \ldots 17$

2-B: Mathematical Description $\ldots \ldots \ldots \ldots \ldots \ldots \ldots \ldots \ldots \ldots \ldots \ldots \ldots \ldots \ldots \ldots \ldots$

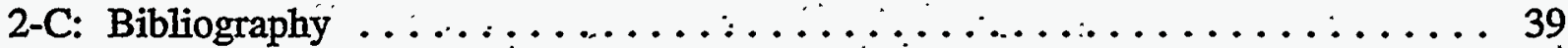

2-D: Model Abstract . ................................ 41

2-E: Data Quality and Estimation Processes $\ldots \ldots \ldots \ldots \ldots \ldots \ldots \ldots \ldots \ldots$

3. Wind Energy Submodule (WES) $\ldots \ldots \ldots \ldots \ldots \ldots \ldots \ldots \ldots \ldots \ldots \ldots \ldots \ldots \ldots$

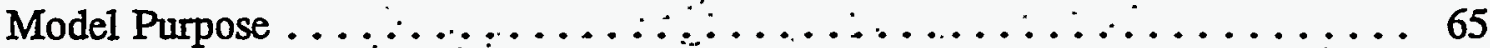

Relationship of the Wind Submodule to Other Models . . . . . . . . . . 65

Modeling Rationale $\ldots \ldots \ldots \ldots \ldots \ldots \ldots \ldots \ldots \ldots \ldots \ldots 6 \ldots \ldots \ldots$

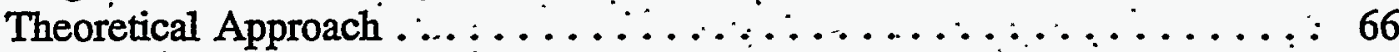

Fundamental Assumptions . . . . . . . . . . . . . . . . . . . 66

Energy Information Administration/NEMS Renewable Fuels Module Documentation Report-Contents I 


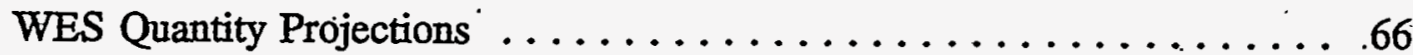

Land Use Estimates . . . . . . . . . . . . . . . . . . . . 67

Dispersed Penetration . . . . . . . . . . . . . . . . 67

Projected Btu Value of Wind Energy $\ldots \ldots \ldots \ldots \ldots \ldots \ldots \ldots \ldots 67$

Alternative Approaches . . . . . . . . . . . . . . . . . . . . 67

Wind Energy Submodule Structure . . . . . . . . . . . . . . . 68

. Submodule Flow Diagram . . . . . . . . . . . . . . . 68

Key Computations and Equations $\ldots \ldots \ldots \ldots \ldots \ldots \ldots 68$

\section{Appendices}

3-A: Inventory of Variables, Data, and Parameters .............. 71

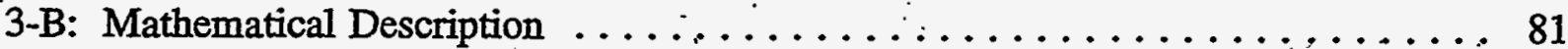

3-C: Bibliography $\ldots \ldots \ldots \ldots \ldots \ldots \ldots \ldots \ldots \ldots \ldots \ldots \ldots \ldots \ldots \ldots \ldots$

3-D: Model Abstract . ......................... 89

3-E: Data Quality and Estimation Processes .................. 93

4. Solar Submodule $\ldots \ldots \ldots \ldots \ldots \ldots \ldots \ldots \ldots \ldots \ldots \ldots \ldots \ldots \ldots$

Model Purpose ............................. 95

Relationship of the Solar Submödule to Other Models . . . . . . . . . 95

Modeling Rationale $\ldots \ldots \ldots \ldots \ldots \ldots \ldots \ldots \ldots \ldots \ldots \ldots . \ldots . \ldots 6$

Theoretical Approach . . . . . . . . . . . . . . . . . . 96

Fundamental Assumptions . . . . . . . . . . . . . . . . . . . 97

Alternative Approaches . . . . . . . . . . . . . . . . . . . . . 97

Solar Submodule Structure $\ldots \ldots \ldots \ldots \ldots \ldots \ldots \ldots \ldots \ldots$. 99

Submodule Flow Diagram $\ldots \ldots \ldots \ldots \ldots \ldots \ldots \ldots \ldots \ldots 9 . \ldots \ldots$

Key-Computations and Equations $\ldots \ldots \ldots \ldots \ldots \ldots \ldots \ldots \ldots$

\section{Appendices}

4-A: Inventory of Variables, Data, and Parameters . . . . . . . . . . . 101

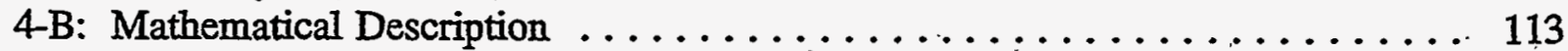

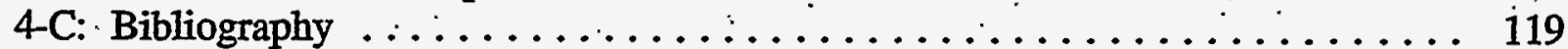

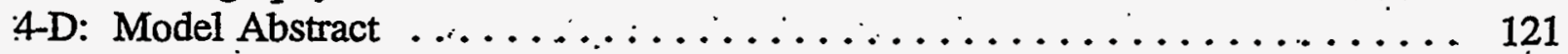

4-E: Data Quality and Estimation Processes. . . . . . . . . . . . . . 125

5. Biofuels Supply Submodnle $\ldots \ldots \ldots \ldots \ldots \ldots \ldots \ldots \ldots \ldots \ldots \ldots \ldots$

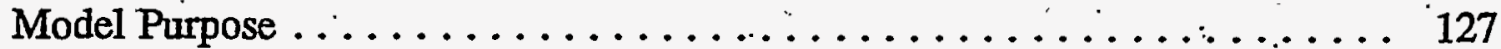

Relationship of the Biofuels Submodule to Other Models . . . . . . . 127

Modeling Rationale . . . . . . . . . . . . . . . ............. 129

Theoretical Approach . . . . . . . . . . . . . . . . . . 129

Fundamental Assumptions . . . . . . . . . . . . . . . . . . 129

Ethanol Production Capàcity . . . . . . . . . . . . . . . ..... 129

Ethanol Production Costs . . . . . . . . . . . . . . . 130

Treatment of Energỳ Crop Ethanol Feedstocks . . . . . . . . . 131

Alternative Approaches $\ldots \ldots \ldots \ldots \ldots \ldots \ldots \ldots \ldots \ldots \ldots \ldots \ldots \ldots$ 
Biofuels Submodule Structure $\ldots \ldots \ldots \ldots \ldots \ldots \ldots \ldots \ldots \ldots \ldots \ldots$

Submodule Flow Diagram $\ldots \ldots \ldots \ldots \ldots \ldots \ldots \ldots \ldots \ldots \ldots \ldots$

Key Computations and Equations $\ldots \ldots \ldots \ldots \ldots \ldots \ldots \ldots \ldots \ldots$

Appendices

5-A: Inventory of Variables, Data, and Parameters . . . . . . . . . . 137

5-B: Mathematical Description $\ldots \ldots \ldots \ldots \ldots \ldots \ldots \ldots \ldots \ldots \ldots \ldots \ldots \ldots \ldots$

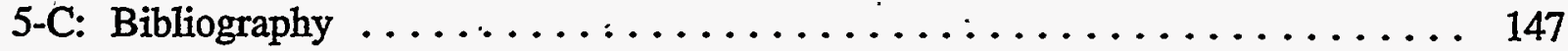

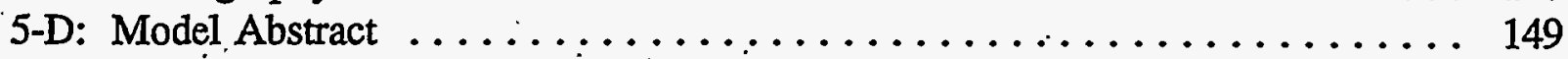

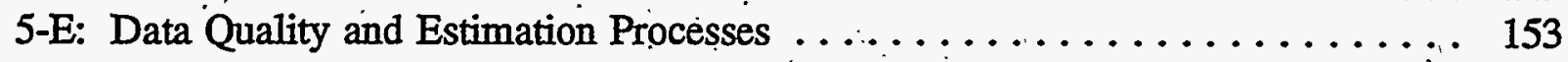

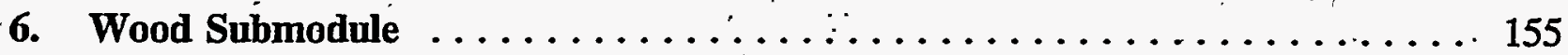

Model Purpose ......................... 155

Model Objectives ......................... 155

Relationship of the Wood Submodule to Other Models . . . . . . . . . 155

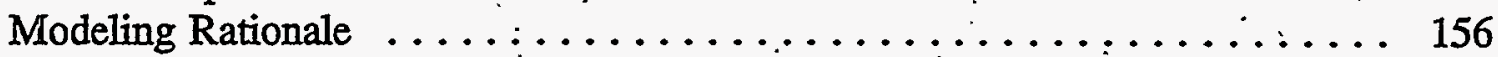

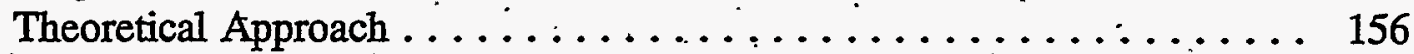

Wood Classification ........................ 156

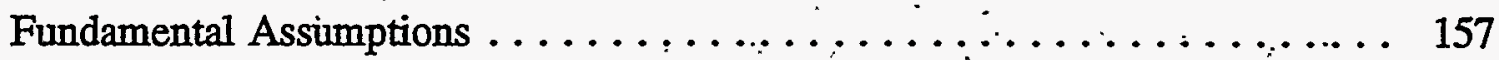

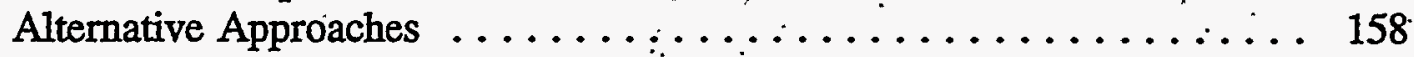

Wood Submodule Structure $\ldots \ldots \ldots \ldots \ldots \ldots \ldots \ldots \ldots \ldots \ldots \ldots \ldots$

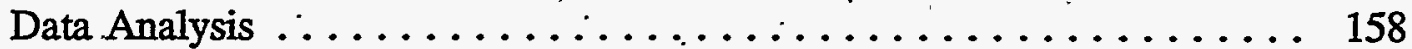

Key Computations and Equations $\ldots \ldots \ldots \ldots \ldots \ldots \ldots \ldots \ldots 1$

\section{Appendices}

6-A: Inventory of Variables, Data, and Parameters . . . . . . . . . . 165

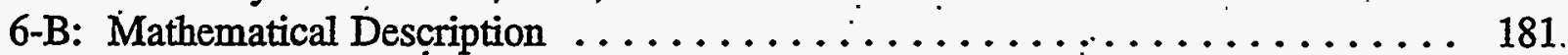

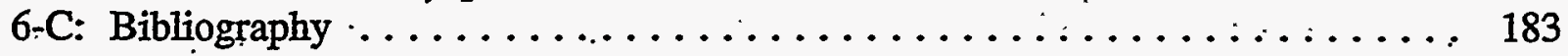

6-D: Model Abstract ........................... 185

6-E: Data. Quality and Estimation Processes $\ldots \ldots \ldots \ldots \ldots \ldots \ldots \ldots \ldots \ldots 187$

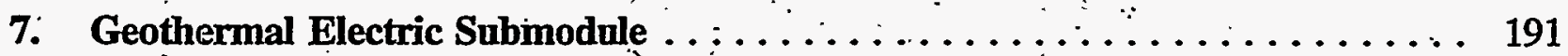

Model Purpose .. . . . . ........................... 191

Relationship of the Geothermal Electricity Submodule to Other Models . . . . 191

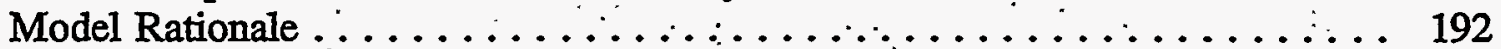

Fundamental Assumptions ......................... 193

Type of Resource $\ldots \ldots \ldots \ldots \ldots \ldots \ldots \ldots \ldots \ldots \ldots \ldots \ldots \ldots$

Conversion Technologies ....................... 193

Drilling and Extraction Technologies . . . . . ............. 194

Alternative Approaches $\ldots . . \ldots \ldots \ldots \ldots \ldots \ldots \ldots \ldots \ldots \ldots \ldots \ldots \ldots .194$

Geothermal Electricity Submodule Structure $\ldots \ldots \ldots \ldots \ldots \ldots \ldots \ldots \ldots$

Submodule Flow Diagrams $\ldots \ldots \ldots \ldots \ldots . \ldots \ldots \ldots \ldots \ldots \ldots 195$

Key Computations and Equations $\ldots \ldots \ldots \ldots \ldots \ldots \ldots \ldots \ldots \ldots$ 


\section{Appendices}

7-A: Inventory of Variables, Data, and Parameters . . . . . . . . . . 219

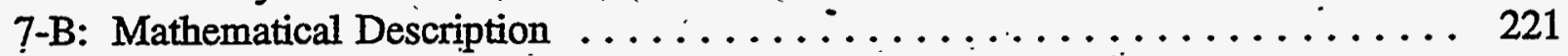

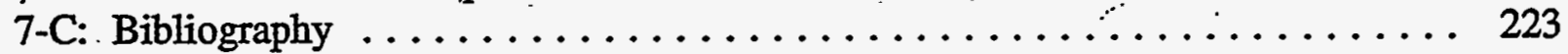

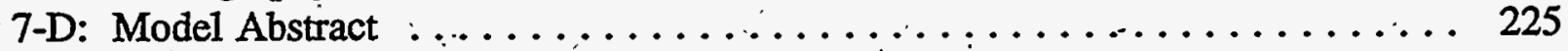

7-E: Data Quality and Estimation Processes . . . . . . . . . . . . . 229 


\section{Tables}

1. EPRI Regional Cost Adjustment Factors . . . . . . . . . . . . . 5

2. NERC Regional Cost Adjustment Factors $\ldots \ldots \ldots \ldots \ldots \ldots \ldots \ldots 6$

3. Com Production Cost Skeleton Matrix (\$/gal) .............. 134 .

4. U.S. Forest Service Wood Resource Categories by Type and

- Demanding Sectors ......................... 157

2A-1. 'NEMS Municipal Solid Waste Submodule Inputs and Outputs $\ldots \ldots \ldots \ldots 18$

2A-2. NEMS Municipal Solid Waste Combustion Fractions . . . . . . . . . 21

2A-3. Heat Content of MSW . . . . . . . . . . . . . . . . . 22

2A-4. Census Division to NERC Region Conversion Factors . . . . . . . . . . 24

2A-5. Percent MSW Combusted ....................... 25

2B-1. Historical MSW Quantity and GNP Values .............. 30

2B-2. Regional Estimates of MSW Combustion Shares, 1990 and 2010 . . . . . 33

2E-1. Plant-Level Data from the GAA Database with Sector Codes Added . . . . . 48

2E-2. Plant-Level Data with Missing Values Filled In and Sector Code Added. . . 53

2E-3. Data Set Used as Input to FORTRAN Program that calculates .

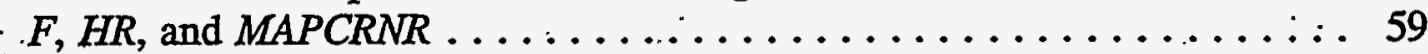

3A-1. NEMS Wind Energy Submodule Inputs and Outputs $\ldots \ldots \ldots \ldots \ldots \ldots 7$

4A-1. NEMS Solar Model Inputs and Outputs . . . . . . . . . . . . 101

4A-2. Selected Photovoltaic Capacity Factor by Modified NERC Region . . . . . 106

4A-3. Selected Solar Thermal Capacity Factors by Modified,NERC Region . . . . 106

5A-1. NEMS Biofuels (Ethanol) Supply Submodule Inputs . . . . . . . . 137

5A-2. NEMS Biofuels (Ethanol). Supply Submodule Outputs $\ldots \ldots \ldots \ldots \ldots \ldots 138$

6A-1. NEMS Wood Submodule Inputs and Variables . . . . . . . . . . 165

6A-2. . Map of Census Divisions to NERC Regions . . . . . . . . . . . . . 167

6A-3a. Supply Schedule for Whole Tree Chips by Census Region . . . . . . . . 169

6A-3b. Supply Schedule for Whole Tree Chips by Census Region . . . . . . . 170

6A-3c. Supply Schedule for Whole Tree Chips by Census Region . . . . . . . . 171

6A-4a. Total Logging. Residue Supply Schedule by Census Regions . . . . . . . . . 172

6A-4b. Total Logging Residue Supply Schedule by Census Regions . . . . . . . . . 173

6A-5. . Mill Residues Available for Energy Use in Noncaptive Markets . . . . . . . 175

6A-6. "Other Wood" Supplies by Census Division $\ldots \ldots \ldots \ldots \ldots \ldots \ldots \ldots \ldots$

6A-7. Heat Rate for Represented Wood Technologies . . ............. 178

6E-1. Aggregated. Supply Schedule for Whole Tree Chips, Logging Residues, Mill Residues, and "Other Wood" . . . . . . . . . . . . . . . 189 


\section{Figures}

1. Municipal Solid Waste Submodule Flowchart $\ldots \ldots \ldots \ldots \ldots \ldots \ldots . \ldots 14$

2. Wind Energy Submodule Flowchart ................... 69

3. Solar Energy Submodule Flowchart ..................... 99

4. Biofuels Supply Submodule Flowchart.$\ldots \ldots \ldots \ldots \ldots \ldots \ldots \ldots \ldots$

5. Wood Submodule Flowchart . . . . . . . . . . . . . . . . 163

6. Geothermal Electricity Submodule Overview Flowchart . . . . . . . . 196

7. GES First Call Initialization Overview Flowchart . . . . . . . . . . . 197

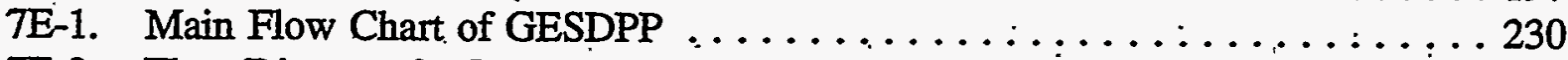

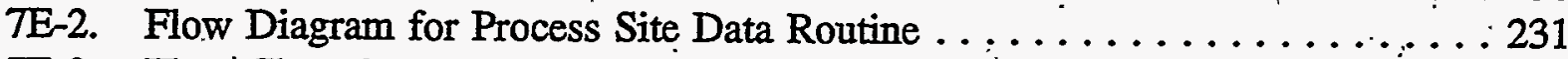

7E-3. Flow Chart for Yplant Routine $\ldots \ldots \ldots \ldots \ldots \ldots \ldots \ldots \ldots \ldots \ldots \ldots \ldots \ldots \ldots \ldots \ldots \ldots \ldots \ldots$ 


\section{Renewable Fuels Module Introduction}

\section{Purpose of This Report}

This report documents the objectives, analytical approach, and design of the National Energy Modeling System (NEMS) Renewable Fuels Module (RFM) as it relates to the production of the 1995 Annual Energy Outlook (AEO95) forecasts. The report catalogues and describes modeling assumptions, computational methodologies, data inputs, and parameter estimation techniques. A number of offline analyses used in lieu of RFM modeling components are also described.

This documentation report serves two purposes. First, it is a reference document for model analysts, model users, and the public interested in the construction and application of the RFM. Second, it meets the legal requirement of the Energy Information Administration (EIA) to provide adequate documentation in support of its models (Public Law 93-275, Federal Energy Administration Act of 1974, Section 57(b)(1). Such documentation facilitates continuity in EIA model development by providing information sufficient to perform model enhancements and data updates as part of EIA's 'ongoing mission to provide analytical and forecasting information systems.

\section{Renewable Fuels Module Summary}

The RFM consists of six analytical submodules that represent each of the major renewable energy resources-wood, municipal solid waste (MSW), solar energy, wind energy, geothermal energy, and alcohol fuels. The RFM also reads in hydroelectric facility capacities and capacity factors from a data file for use by the NEMS Electricity Market Module (EMM). ${ }^{1}$

The purpose of the RFM is to define the technological, cost and resource size characteristics of renewable energy technologies. These characteristics are used to compute a levelized cost to be competed against other similarly derived costs from other energy sources and technologies: The competition of these energy sources over the NEMS time horizon determines the market peentration of these renewable energy technologies. The characteristics include available energy capacity, capital costs, fixed operating costs, variable operating costs, capacity factor, heat rate, construction lead time, and fuel product price. :

\footnotetext{
${ }^{1}$ Hydroelectric capacity additions and capacity factors are derived from EIA survey data. Although the RFM reads these data' for use by the EMM, the data reside in the EMM utility plant file, an exogenous input file that provides plant-specific construction, cost, and operating parameters. The utility plant file is described in the EMM documentation report. "Since hydroelectric power is not competed against any other energy form for utility capacity expansion, and all the hydroelectric generation is consumed because it is generally the lowest cost, there is not need to have an interactive hydroelectric submodule. It was determined that a data file with maximum capacity, capacity factors, and the other items contained in the file would most accurately represent hydroelectric power in NEMS."
} 
Currently, the RFM is structured principally to provide the EMM with technology and cost data for central station electric generation facilities. One exception is the MSW Submodule, which generates MSW energy consumption values for several end-use demand models along with the EMM. However, the demand models are not currently configured to access these data. Another exception is the Biofuels Submodule, which provides ethanol supply curves for the Petroleum Market Module (PMM).

Other renewables modeled elsewhere in NEMS include the biomass in the industrial sector; wood in the residential sector, geothermal heat pumps in the residential and commercial sectors; and solar hot water in the residential sector. Thus there are several areas, primarily dispersed application, that are not represented in NEMS. This includes direct applications of geothermal heat, several types of solar thermal use, and photovoltaics. For the most part, the expected contributions from these sources are small; however, there are preliminary plans to add many for future analyses.

The number and purpose of the associated technology and cost characteristics varies from one RFM submodule to another depending on the modeling context. For example, renewable resources such as solar, wind, and geothermal energy are not fuels; rather, they are "costless" inputs to electricity or heat conversion processes. Consequently, the Solar, Wind, and Geothermal Submodules do not provide fuel product prices. As another example, the MSW Submodule's capital and operating cost characterization is used by the NEMS Electricity Market Module (EMM) solely to help determine electricity prices. Unlike the other RFM technology. characterizations, the MSW-to-energy facility characterization is not used to compete MSW energy against other energy sources. 'This modeling treatment stems from the assumption that MSW energy, as a byproduct of the waste removal process, is fully utilized as it is produced.

Several sources for the cost and performance characterization data were examined for use in the RFM. Among these were the technology characterizations developed by the DOE Office of Energy Efficiency and Renewable Energy (EE), data inputs to the DOE Policy Office's Integrated Dynamic Energy Analysis Simulation (IDEAS) model, and the Electric Power Research Institute's 1993 Technical Assessment Guide (EPRI TAG): The IDEAS data are older, and are not consistent with some RFM variable categories. The EPRI TAG data was chosen as the most credible and consistent data for most technologies; however, in some cases EE data were used.

The sources provide values for capital costs (excluding the construction financing and contingency components, since these are provided in the EMM), fixed and variable operation \& maintenance (O\&M) costs, capacity factors for solar electric technologies, and construction lead times. All cost values are converted to real 1987 dollars.

All information passed from the RFM to other NEMS modules are placed in a COMMON block called /WRENEWI. (A COMMON block is a FORTRAN data area located outside of the NEMS. analytical modules). It should be noted that no data are passed among the RFM submodules; that is, there are no variables written into COMMON /WRENEW/ by an RFM submodule that are subsequently read by any of the other RFM submodules. 
Provided below are summaries of the six RFM submodules that are used for producing the AE095 forecasts: the Municipal Solid Waste Submodule (MSW), the Wind Energy Submodule (WES), the Solar Energy Submodule (SOLES), the Biofuels Supply Submodule (BSS), the Wood Submodule, and the Geothermal Electricity Submodule (GES). The RFM's role in providing the EMM with hydropower data are also described. The chapter concludes with information on the RFM archival package and EIA point of contact.

\section{Municipal Solid Waste Submodule (MSW)}

The Municipal Solid Waste Submodule provides annual projections of energy produced from the incineration of municipal solid waste (MSW). The Submodule uses the quantity of MSW produced (derived from an econometric equation that uses Gross Domestic Product as the principal forecast driver), the heating value of a pound of MSW, and shares of MSW combusted for energy recovery. The energy production forecasts are disaggregated by consuming sector (commercial, industrial, and utility). In addition, the MSW Submodule supplies the utility sector. (EMM) with capital and operating cost information. This cost information is only used by the EMM to calculate electricity prices; MSW-produced power is viewed as a byproduct of a community's waste disposal activities and only secondarily as a competitive alternative to other fuels for energy production.

\section{Wind Energy Submodule (WES)}

The Wind Energy Submodule (WES) projects the availability of wind resources as well as the cost and performance of wind turbine generators. This information is passed to the EMM so that wind turbines can be built and dispatched in competition with other electricity generating technologies. The wind turbine data are expressed in the form of energy supply curves. The supply curves provide the maximum amount of turbine generating capacity that could be installed, given the available land area, average wind speed, and capacity factor. These variables are passed to EMM in the form of nine time slices which are matched to electricity load curves within EMM.

\section{Solar Submodule (SOLES)}

The solar submodule projects the cost and performance characteristics of photovoltaic (PV) and solar thermal central station electric installations. (The submodule considers only grid-connected applications constructed by a utility or independent power producer.) This information is passed to the EMM for building and dispatching these solar technologies in competition with other electricity generation technologies.

\footnotetext{
${ }^{2}$ The nine time slices are derived from three 8-hour segments of the day for three seasons-winter, summer and off-peak (spring/fall averaged). The data represent average capacities based on empirical analysis.
} 
The required input information is similar for each type. Capacity projections are developed as endogenous inputs to be competed against other generating technologies (i.e., costs, capacity factors, and fixed and variable O\&M costs). Solar is a renewable energy form that requires a more detailed characterization to represent its intermittent nature and regionality. This is dealt with by the regional load shapes used by the EMM and different time slices to represent intermittency (for example, that there is no sunlight at night). Little if any solar capacity is being built currently because of the high cost.

\section{Biofuels Supply Submodule (BSS)}

This submodule produces annual supply functions (cost vs. quantity) by Petroleum Allocation for Defense Districts (PADD) and by -Census division for corn-derived ethanol. The agricultural feedstock production quantities and costs are provided exogenously to NEMS from a U.S. Department of Agriculture linear programming model, Agricultural Resources Interregional Modeling System (ARIMS). The supply curves take into account feedstock costs, feedstock. conversion costs, and energy prices. The supply functions are used by the Petroleum Market Module to compute regional demands for ethanol.

\section{Wood Submodule}

The Wood Submodule furnishès cost and performance chäracteristics for a wood burning electricity generating technology to the EMM. The technology modeled for the AEO95 is the Integrated Gasification Combined Cycle (IGCC). The submodule utilizes a regional wood supply schedule from which the wood fuel price is determined; fuel prices are added to variable operating costs since there are no fuiel costs in the structure of NEMS for renewable fuels. The - wood supply schedule is based on the accessibility of wood resources by the consuming sectors from existing timber and wood residues. For the AEO95 energy crops are not included: There are plans for their inclusion in AEO96.

\section{Geothermal Electricity Submodule (GES)}

The purpose of the GES is to model current and future regional supply, capital cost, and operation and maintenance costs of electric generating facilities using hydrothermal resources (hot water and steam). These resources are limited to three EMM regions in the western United States $(11,12$ and 13). The data are assembled from specific site information which reflects the conditions of that location. Capital and operating costs can vary among sites. For AEO95 a nonintegrated version of the GES was used which relied on the data structure within the GES to produce supply schedules which corresponded to levelized costs of $\$ .05 / \mathrm{kWh}$ or less. These costs parameters were then entered into the appropriate areas of the RFM for inclusion in EMM capacity expansion decisions. The components of GES that were required for AEO95 are documented in chapter 7 : 


\section{Hydroelectric Plant Data}

The EMM úses currently available and planned regional hydropower capacity and capacity factors for modeling conventional hydroelectric facility utilization. This plant information is derived by processing and aggregating the responses of utility and nonutility power producers to annual EIA power plant surveys (Forms EIA-860, EIA-759, and EIA-867). Prior to calling the renewable energy submodules, the RFM reads the capacity and capacity factor values from the EMM plant file into the WRENEW COMMON block variables (hydroelectric capacity, WCAHYEL, and capacity factor, WCFHYEL). These COMMON block variables are subsequently used by the EMM .Electricity Fuel Dispatch (EFD) and Electricity Capacity Planning (ECP) submodules.

In addition to capacity and capacity utilization inputs, the EMM also requires capital costs and fixed and variable operation \& maintenance costs for the calculation of electricity prices in the Electricity Finance and Pricing (EFP) submodule. These inputs, which come from the EPRI TAG, are contained in an input table named TECHP, located in the RFM source code: Table TECHP has the following hydroelectric plant parameters which pertain to the EPRI West region: Capital Cost- $\$ 1,849$ perkilowatt; Fixed Operating Cost $-\$ 10.2$ per kilowatt; Variable Operating Cost-3.2 mills per kilowatthour.

These EPRI regional values need to be converted into NEMS Electricity Supply (NERC) regions. The model accomplishes this conversion in three steps. In step one, a set of six EPRI TAG regional cost adjustment factors are mapped to NERC regions with the use of a mapping array; MAPEN. The regional cost adjustment factors, located in the RFM source code as table TECHF, are as shown in Table 1:

Table 1. EPRI Regional Cost Adjustment Factors

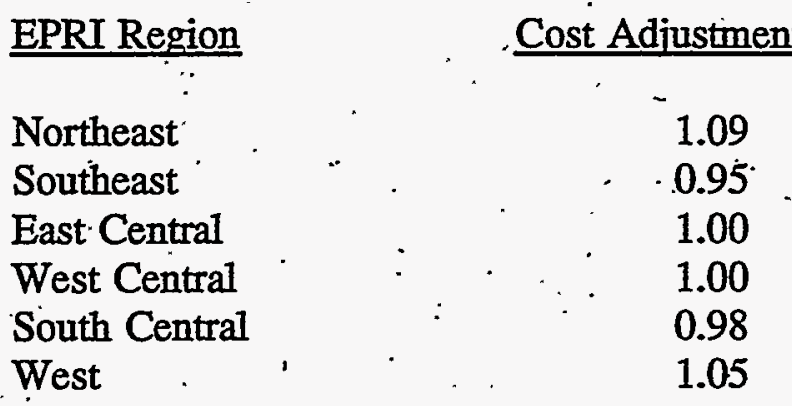

In the NERC numbering system, this corresponds to the values in Table 2: 
'Table 2. NERC Regional Cost Adjustment Factors

NERC Region

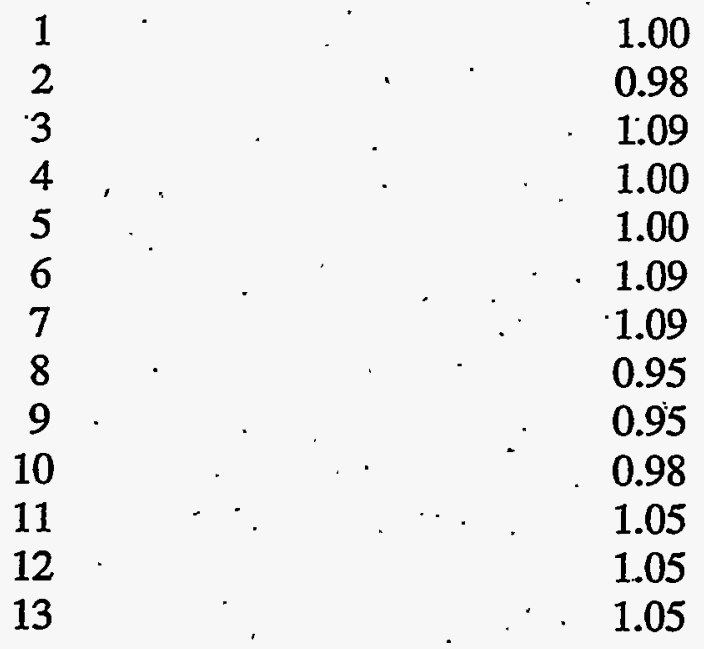

In step.two; the 13 cost adjustment factors are divided by the cost adjustment factor for the EPRI West region, where the EPRI TAG prototype hydroelectric plant was located.

Finally, these normalized cost adjustment ratios are multiplied by the elements in TECHP to obtain the regionalized EPRI TAG plant cost parameters.

Hydropower modeling in NEMS focuses only on planined hydroelectric capacity additions and reductions through the year 2001 (the end point of the 1991 EIA-860 survey 10-year planning horizon). Unplanned capacity changes beyond 2001 are assumed to offset one another, resulting in no net change in hydroelectric capacity for the duration of the forecast horizon. Since hydropower O\&M costs are assumed to be the lowest of any major generating technology, and hydropower produces virtually no air pollution, all available hydro capacity is used first by the EMM.

\section{Archival Media}

The RFM is archived as part of the National Energy. Modeling System production runs: 


\section{Model Contact}

Perry M. Lindstrom, Economist

Coal, Uranium, and Renewable Fuels Analysis Branch,

Energy Supply and Conversion Division,

Energy Information Administration, 1000 Independence Ave., S.W.

Washington, DC 20585

Phone: (202) 586-0934

\section{Report Organization.}

Subsequent chapters of this report provide detailed documentation of each of the RFM's six working submodules. Each chapter contains the following sections:

- Model Purpose-a summarization of the submodule's objectives, detailing input and output quantities, and the relationship of the submodule to other NEMS modules

- Model Rationale-a discussion of the submodule's design rationale, including insights into assumptions utilized in the model development process, and alternative modeling methodologies considered during submodule development phase

- Model Structure-an outline of the model structure, using text and graphics to illustrate the major model data flows and key computations

Appendices-supporting documentation for input data and parameter files currently residing on the EIA mainframe computer: Appendix A in each RFM submodule chapter lists and defines the input data used to generate parameters and endogenous forecasts. Appendix B contains a mathematical description of the computation algorithms, including model equations and variable transformations. Appendix $C$ is a bibliography of reference materials used in the model development process. Appendix D consists of a model abstract. Appendix E discusses data quality and estimation methods. 



\section{Municipal Solid Waste (MSW) Submodule}

\section{Model Purpose}

The main purpose of the Municipal Solid Waste (MSW) Submodule is to provide NEMS with annual projections of energy produced from the incineration of municipal solid waste (MSW). The submodule uses the quantity of MSW produced, the heating value of MSW, and shares of MSW combusted for energy recovery to produce forecasts of the production of electricity and other energy forms. The energy production forecasts are disaggregated by consuming sector (commercial, industrial, and electric utility) and region.

In addition to energy production forecasts, the MSW Submodule supplies NEMS with waste-toenergy (WTE) facility capital and operating costs. For policy analysis, the Submodule contains a policy variable that models the effects of MSW source reduction efforts.

\section{Relationship of the MSW Submodule to Other Models}

Unlike most of the submodules of the Renewable Fuels Module, the MSW Submodule does not provide other NEMS modules with information used to allow the renewable energy resource to compete with alternative energy forms. Rather, forecasted MSW energy production is used to - reduce the energy demand that is modeled in the NEMS end-use demand and utility modules (i.e., MSW energy is decremented from each sectoral energy requirement; in the case of the electric utility sector, generating capacity is decremented). This treatment of MSW energy production in NEMS stems from MSW energy being viewed primarily as a byproduct of a community's waste disposal activities rather than a competitive alternative to other fuels.

The only input from other NEMS modules is annual real Gross Domestic Product (GDP); which comes from the NEMS Macroeconomic Activity Module (MAM) variable COMMON block (variable name MC_GDP).

The outputs to the NEMS commercial, industrial, and electric utility modules include the following:

- - Quantities of total energy produced annually by, WTE facilities for each region and enduse sector. For the NEMS industrial and commercial sector modules, energy forecasts are provided in thermal units (million Btu) by Census division. For the Electricity Market Module (EMM), energy forecasts are converted to megawatt-electric equivalents and reported by NERC region.

- Capital cost (plant and startup costs, plus interest during construction), variable operating cost, and fixed operating cost for WTE facilities, to be used by the EMM for calculating purchased electricity costs. 


\section{Modeling Rationale}

\section{Theoretical. Approach}

The modeling methodology employs a simple linear MSW supply function and multiplicative energy. allocation shares for deriving disaggregated MSW energy production forecasts. The methodology consists of four major steps. First, the total quantity of MSW in the United States is projected using univariate regression estimation to derive parameters for the MSW supply. equation (an add factor representing the impact of MSW. source reduction is also included in the equation). Second, the current and future heat value of a typical pound of MSW is assessed for estimating the potential quantity of energy that can be produced from combusting MSW. Third, estimates of the total U.S. capacity to burn MSW with heat recovery are obtained using analyst judgement of factors affecting community approval and investments in WTE facilities. Fourth, regional and sectoral projections of energy from MSW combustion are obtained by multiplying together MSW quantities, Btu heating values, percentages of MSW combusted, and sectoral/regional energy allocation shares.

While this approach is essentially unchanged from the methodology used for previous AEO forecasts, there are some new features that have been incorporated into the NEMS version. First, the MSW supply forecasting equation, which is based on GDP, will access that variable directly from the NEMS Macroeconomic Activity Module and thus be consistent with economic assumptions used throughout NEMS. Previously; MSW supply projections were based on off-line forecasts of economic growth. Second, MSW energy projections will make the transition from the ten Federal regions utilized in the AEO Forecasting System to NEMS regions (Census Divisions and NERC regions). Third; the flexibility with which important input variables can be changed, such as the assumed percentage of MSW that is combusted, has been improved.

Because of the byproduct nature of MSW energy, the relatively small quantity of MSW in the U.S. energy mix, and the complexity of modeling the municipal WTE market, a simple modeling approach that excludes the consideration of energy demand, price, and technology investment signals from other NEMS modules was selected. One of the major limitations of this approach is that there are no economic or financial links for determining key parameters, especially the share of MSW combusted and the regional distribution of WTE energy capacity.

\section{Fundamental Assumptions}

\section{MSW Quantity Projections}

The definition of MSW adopted for the MSW Submodule is consistent with that used by the Environmental Protection Agency (EPA) and defined in Subtitle D of the Resource Conservation and Recovery Act. Municipal solid waste includes discarded durable goods, nondurable goods, containers and packaging, food wastes, and yard trimmings from the residential, commercial, institutional, and industrial sectors. MSW does not include everything that might be landfilled in Subtitle D landfills, such as municipal sludge, nonhazardous industrial wastes, shredder residue 
from automobile recycling operations, and construction and demolition wastes. These wastes are often disposed alongside those wastes formally defined as MSW. The MSW Submodule does not currently represent energy that may be generated from the combustion of Subtitle D wastes other than MSW:However, it is possible that some existing capacity could be used for combustion of these additional Subtitle D wastes.

\section{Projected Btu Value of MSW}

The Btu value of a typical pound of MSW is changing rapidly in response to changes in the usage and disposal of specific materials. Curlee (1992) provides information on the historical and projected composition of MSW in terms of the waste stream's material composition. In this estimate, the Btu value of one pound of MSW has increased from about 3,800 Btu in 1960 to about 5,100 Btu in 1990.

There are numerous factors that influence the Btu value of combusted MSW. For example, marketing efforts have been responsible for the gradual replacement of glass and some metal with plastic, especially for containers. Partially counteracting these marketing efforts are restrictions that have been successfully implemented in some States to limit the usage of plastics in selected packaging. Many communities require that yard waste (which has a low energy content) to be collected separately from other wastes and composted. rather than burned or landfilled. Other communities simply restrict households from disposing of their yard waste along with other MSW. The number of curbside recycling programs is increasing, and most collect and recycle both plastics and paper (the highest Btu components of the waste stream), and glass and metals (which have no energy value)..

Combining EPA projections of this changing MSW mix with the heat content of waste components, Curlee projects a total heat content for MSW of 5,569 Btu per pound of waste in the year 2000. It was assumed by EIA that post-2000, the heat content would remain constant at the 5,569-Btu level, based on the expectation that the removial of low-Btu waste stream components (metal, glass, and yard waste) will be balanced by the removal of high-Btu components (paper and plastic). While these changes in MSW composition are-significant, it is not believed that the relationship of GDP to tons generated will be affected by such changes.

\section{Projected Percentage of MȘW Combusted With Heat Recovery}

Projections of WTE market penetration, and therefore the share of generated municipal waste combusted, are difficult to make. Projections for the near term-i.e., the next 5 years - can be based on existing data on WTE projects in the planning and construction phases. Consideration should be given to-expected unit cancellations, which have occurred more frequently in recent years. The methodology adopted for the MSW Submodule beyond 1995 requires the use of assumed fractions of MSW supplies combusted for energy recovery. The procedure used to derive these combusted-fraction values is described in Appendix 2E, page 25. 


\section{Disaggregation Rules}

National projections for energy from MSW are disaggregated into regional totals according to the geographical dispersion of current and planned WTE facilities, use of the MSW energy (electricity versus steam and heat), and sector (commercial, industrial, and utility). Information used for disaggregating MSW energy comes from the Government Advisory Associates (GAA) Resource Recovery Database. This proprietary database product.includes information on locations, types of energy produced, ownership type, etc. for all existing U.S. WTE facilities, as well as those facilities in the construction, conceptual-planning, and advanced-planning stages.

For 1990 to 1996, the regional, usage, and sectoral totals are computed using GAA's site-level facility data. The Btu capacities of WTE facilities that are expected to come on-line before 1996 are assigned to the appropriate NEMS regions: Specifically, MSW electricity production for sales are disaggregated by NERC regions, and energy derived for heat and steam are disaggregated by Census divisions.

Given that no data currently exist to indicate how these breakdowns may change beyond 1996, it is assumed that the percentage of total WTE capacity allocated to region, use, and sector remains constant after 1996: Additional research into regional characteristics that could influence the shares, such as land values or recycling markets, may result in an improved approach for disaggregation of national totals.

\section{Capital and Operating Costs}

The MSW submodule supplies the EMM with capital and operating costs to help in the determination of electricity prices. In lieu of actual cost data from WTE facilities, the MSW. Submodule employs technology cost characterization information from the EPRI 1989 Technical Assessment Guide (TAG). Information for the mass burn technology is selected because this technology is the most common of three techriology types. ${ }^{3}$ For both capital and operating costs, the TAG assumes a WTE planit șize of 40 megawatts with a single combustion unit. Additional information on the EPRI TAG cost values is provided in Appendix 2A.

An important component of the WTE facility operating cost is the tipping fee. The tipping fee is a per-ton charge assessed to waste removal firms for depositing the MSW at the disposal site. Because the tipping fee is a revenue source, the MSW Submodule treats the tipping fee as a negative fuel cost.

At this time there are insufficient data on how tipping fees are determined, although it is likely that they are the balancing factor in plant economics. A complication with tipping fees is that

\footnotetext{
${ }^{3}$ Mass bun WTE units combust MSW without preprocessing, other than the removal of large items from the feed system. Refuse Derived Fuel (RDF) facilities combust waste that has been preprocessed (i.e., soited and shredded to increase the heating value). A third technology type-modular combustors-are small, prefabricated units. Mass burn mits constitute 39 percent of operating WTE units and 79 percent of planned WTE units.
} 
" some plants are privately owned, others are publicly owned, and subsidies may be involved in either case. As a result, tipping fee values are currently assumed to remain constant for all forecast years.

\section{Alternative Approaches}

Only two other sources of energy projections from MSW combustion have been identified - the Solar Energy Research Institute (SERI, 1990) and Klass (1990). The projections from those reports are discussed in Curlee (1991). MSW energy projections given in Curlee (1991), which are based on the methodology adopted for the MSW Submodule, are significantly higher than those contained in the reports by SERI and Klass. Note that MSW is one of several renewable energy sources evaluated in both the Klass and SERI studies, and the underlying assumptions and modeling methodologies are not explained sufficiently in either study to discuss and compare the differences between their approaches and the MSW Submodule approach. No other models of MSW energy consumption and production were identified in the research supporting the development of the MSW Submodule.

A key aspect of the selected modeling approach involves the application of expert judgement for specifying the projected regional fractions of MSW combusted for energy. (These fractions are multiplied by available MSW in order to determine the projected MSW quantities available for energy recovery.) Ideally; judgements concerning projected combustion fractions should be combined with an analysis of cost and capacity trends involving the reduction, recycling, composting, landfilling, processing, and combusting of MSW. However, relative cost information for the various alternatives to manage $\mathrm{MSW}$ is currently considered inadequate, and is therefore not used.

\section{MSW Submodule Structure}

\section{Submodule Flow Diagram}

This section presents a flow diagram of the MSW Submodule that shows the Submodule's main computational steps and data relationships. 
Figure 1. Municipal Solid Waste Submodule Flowchart

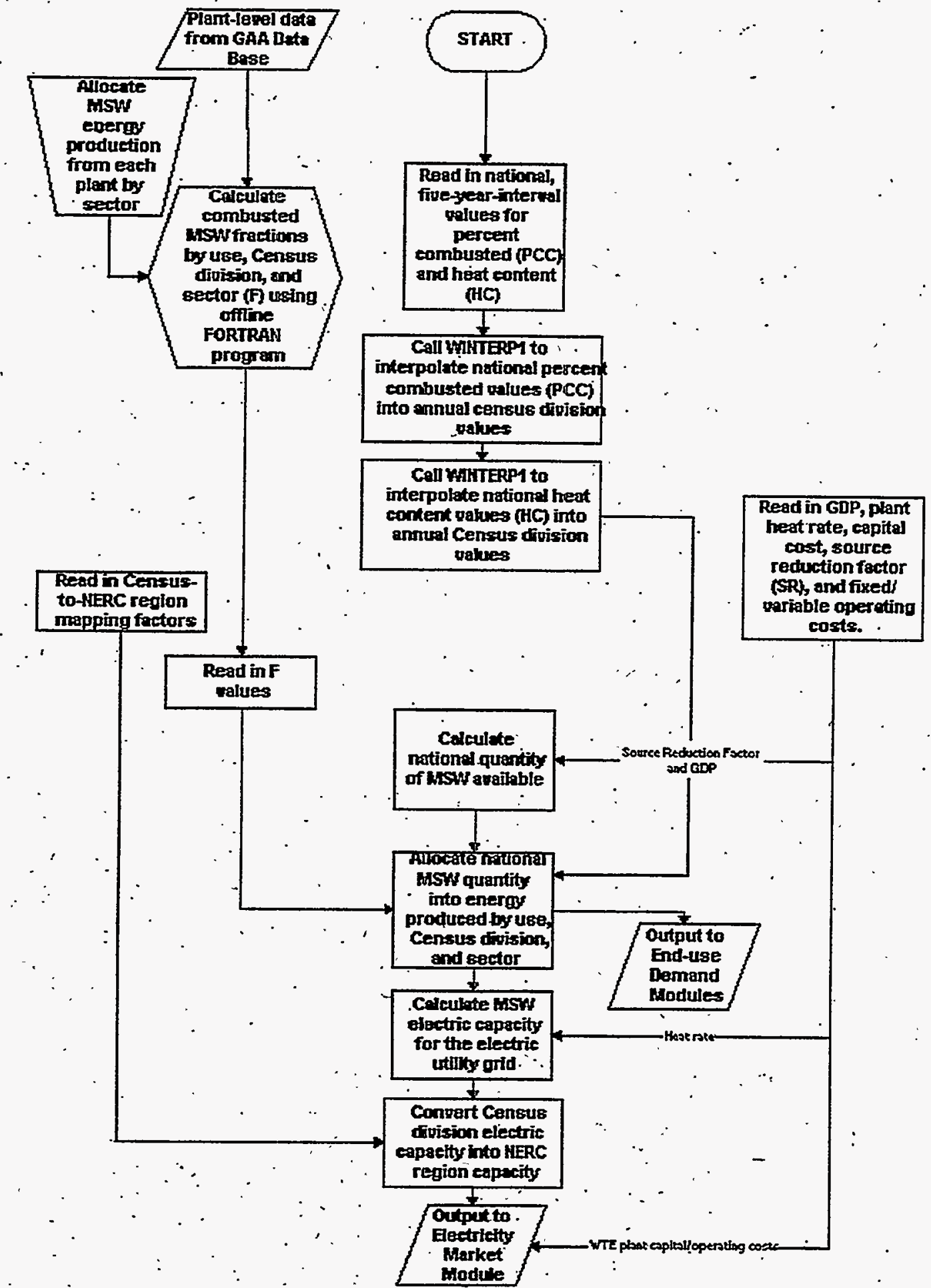




\section{Key Computations and Equations}

The MSW Submodule first computes the annual amount of MSW, in a direct linear relationship with economic activity as represented by GDP, and adjusted for efforts to reduce MSW. The quantity, in million tons, that is generated nationally is:

$$
Q N A T=\alpha+\beta x M C_{-} G D P_{T} x[1-(T-1) x S R]
$$

where:

$$
\begin{aligned}
& \text { QNAT = Quantity of municipal solid waste generated in the } \\
& \text { United States, this variable is for internal use only } \\
& \text { and is overwritten on each iteration, } \\
& \alpha \quad=\quad \text { an econometrically estimated parameter equal to } \\
& 14.60 \text {, } \\
& \beta \quad=\quad \text { an econometrically estimated parameter equal to } \\
& 0.03599 \text {, } \\
& T \quad \cdot=\quad \text { current NEMS year, } \\
& M C_{-} G D P_{T} \quad=\quad \text { real gross domestic product for year } T \text {, and, } \\
& S R . \quad . \quad=\text { annual source reduction factor. }
\end{aligned}
$$

Additional detail on the estimation of $\alpha, \beta$ and SR is given in Appendix 2-B. The next step is to compute the amount of energy obtained from the national MSW supply quantity, and disaggregate the energy into usage, regional, and sectoral shares. ${ }^{4}$ The following equation determines the quantity shares of energy, measured in millions of Btu per year, that is produced from MSW:

$$
Q_{U, R C, S}=Q N A T \times P C C_{R C, T} \times H C_{R C, T} \times F_{U, R C, S} \times 2000
$$

where:

$Q_{v_{\perp, R C S}} \quad . \quad=\quad$. $\quad$ quantity of energy produced from municipal solid waste for use $U$ (where $U_{I}=$ electricity supplied to the grid, $U_{2}=$ electricity supplied for own use, and

${ }^{4}$ All energy produced from MSW is assmed to be used by the three consuming sectors. 
$U_{\hat{3}}=$ steam and other), sector $S$ (where $S_{1}=$ commercial sector, $S_{2}=$ industrial sector, and $S_{3}=$ electric utility sector), in Census division $R C$,

QNAT = quantity of municipal solid waste generated in the United States,

$P C C_{R C T}, \quad=\quad$ percent combusted for Census division $R C$ in year $T$,

$H C_{R C T} \ldots \quad . \quad=\quad$ heat content for Census division $R C$ in year $T$, and

$F_{U, R C S S} \quad=\quad$ fraction of MSW combusted for use $U$ in sector $S$ in Census division $R \dot{C}$.

The production capacity for all energy forms (electricity and other) is then combined into a total energy production capacity for the commercial and industrial sectors. The annual electric capacity available for MSW facility own-use and sale to the electric utility grid is calculated using the following equation:

$$
\text { WCAMSELRC } C_{R C, T}=\frac{\left[\sum_{U=1}^{2} Q_{U, R C, S=3}\right] \times 10^{6}}{H R I \times 8760 \times 1000}
$$

where:

$$
\begin{array}{ll}
\text { WCAMSELRC } & =\begin{array}{l}
\text { Annual MSW electric capacity in megawatts for } \\
\text { own-use and sale to the utility grid in Census }
\end{array} \\
\text { division } R C \text { in year } T
\end{array}
$$

$H R 1 \quad=\quad$ Heat rate for MSW (Btu in $/ \mathrm{kWh}$ out), and,

$Q_{U, R C S=3} \quad=\quad$ Annual electricity production from MSW in millions of Btu for both sale to the grid $(U=1)$ and own-use $(\mathrm{U}=2)$, in the electric utility sector $(\mathrm{S}=3)$; in Census division $R C$.

Finally, the annual electricity production is mapped from Census divisions to NERC regions using a mapping matrix. 


\section{Appendix 2-A: Inventory of Variables, Data, and Parameters}

This Appendix describes the variables, parameter estimates, and data inputs associated with the MSW Submodule. Table 2A-1 provides a tabular listing of model variables, input data, and parameters. The table contains columns with information on item definitions, modeling dimensions, data sources, measurement units, and documentation page references.

The remainder of Appendix 2A consists of detailed descriptions of data inputs and variables, inclúding discussions on supporting data assumptions and transformations. 
Table 2A-1.' NEMS Municipal Solid Waste Submodule Inputs and Outputs

\begin{tabular}{|c|c|c|c|c|}
\hline 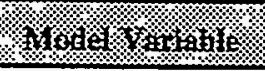 & 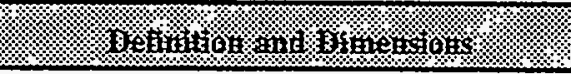 & 3inntix & 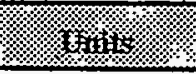 & 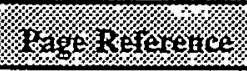 \\
\hline INPUT DATA & & - & & - \\
\hline$F$ & $\begin{array}{l}\text { Fraction of MSW combusted for use } U \\
\text { and sector } \bar{S} \text { in Census division } R C \\
\qquad:\end{array}$ & $\begin{array}{l}\text { Government } \\
\text { Advisory } \\
\text { Associates. } \\
\text { processed using off- } \\
\text { line Fortran program }\end{array}$ & Unitless & $\begin{array}{l}20,24,34 \\
59,62\end{array}$ \\
\hline HC & $\begin{array}{l}\text { MSW heat content values in Census } \\
\text { division } R C \text { in year } T \\
\end{array}$ & $\begin{array}{l}\text { Franklin Associates } \\
\text { and Office of } \\
\text { Technology } \\
\text { Assessment }\end{array}$ & $\begin{array}{l}\text { Btu/lb of } \\
\text { MSW }\end{array}$ & $16,22,30$ \\
\hline HR1 & MSW heat rate for electricity production & $\begin{array}{l}\text { Government } \\
\text { Advisory Associates }\end{array}$ & $\begin{array}{c}\text { Btu in } \\
\mathrm{kWh} \text { out }\end{array}$ & $16,23,31$. \\
\hline MPCRNR & $\begin{array}{l}\text { Conversion factors for converting Census } \\
\text { division } R C \text { to NERC Region } R N\end{array}$ & $\begin{array}{l}\text { Government } \\
\text { Advisory Associates }\end{array}$ & Unitless & $20,23,32$ \\
\hline$P C C$ & $\begin{array}{l}\text { Percent combusted for Census division } \\
R C \text { in year } T \\
\ddots \\
\cdot\end{array}$ & $\begin{array}{l}\text { Franklin Associates } \\
\text { and EIA staff }\end{array}$ & $\begin{array}{l}\text { Percentage } \\
\text { (converted } \\
\text { to a factor } \\
\text { by dividing } \\
\text { by 100) }\end{array}$ & $16,24,30,66$ \\
\hline RCAPFEL & Capacity factor of a WTE plant & EPRI TAG. & Unitless & 25 \\
\hline$R C A P$ & Capital cost for a WTE plant & EPRI TAG & $\$ / K W$ & 26 \\
\hline RNCFUEL & $\begin{array}{l}\text { Tipping fee for MSW in Census division } \\
R C\end{array}$ & Chupka, et al & - \$/ton & 26 \\
\hline$R C O M$ & Fixed O\&M cost for a WTE plant & EPRI TAG & $\operatorname{mills} / \mathbf{k W h}$ & 27 . \\
\hline . .RVOM & Variable O\&M cost for a WTE plant & EPRI TAG & mills/kWh & 27,37 \\
\hline$S R$ & Annual soirce reduction factor & $\begin{array}{l}\text { Oak Ridge National } \\
\quad \text { Lab } \\
\end{array}$ & $\begin{array}{c}\text { Percentage } \\
\ldots \\
\end{array}$ & $15,28,29,62$ \\
\hline $\begin{array}{l}\text { CALCULATED } \\
\text { VARIABLES } \\
\end{array}$ & - & NA* & & \\
\hline$M C_{-} G D P$. & Real gross domestic product for year $T$ & $\cdot$ & Billion \$ & 15,29 \\
\hline$Q$ & $\begin{array}{l}\text { Quantity of energy from municipal solid } \\
\text { waste for use } U \text { and sector } S \text { in Census } \\
\text { Division } R C\end{array}$ & & $\begin{array}{l}\text { MMBtu per } \\
\text { year }\end{array}$ & $15,16,30,31$ \\
\hline QNAT & $\begin{array}{l}\text { Quantity of municipal șolid waste } \\
\text { produced in the U.S. }\end{array}$ & $\ddots$ & $\begin{array}{l}\text { million tons } \\
\text { per-year }\end{array}$ & $15,16,29,30$ \\
\hline
\end{tabular}


Table 2A-1. NEMS Municipal Solid Waste Submodule Inputs and Outputs (Continued) :

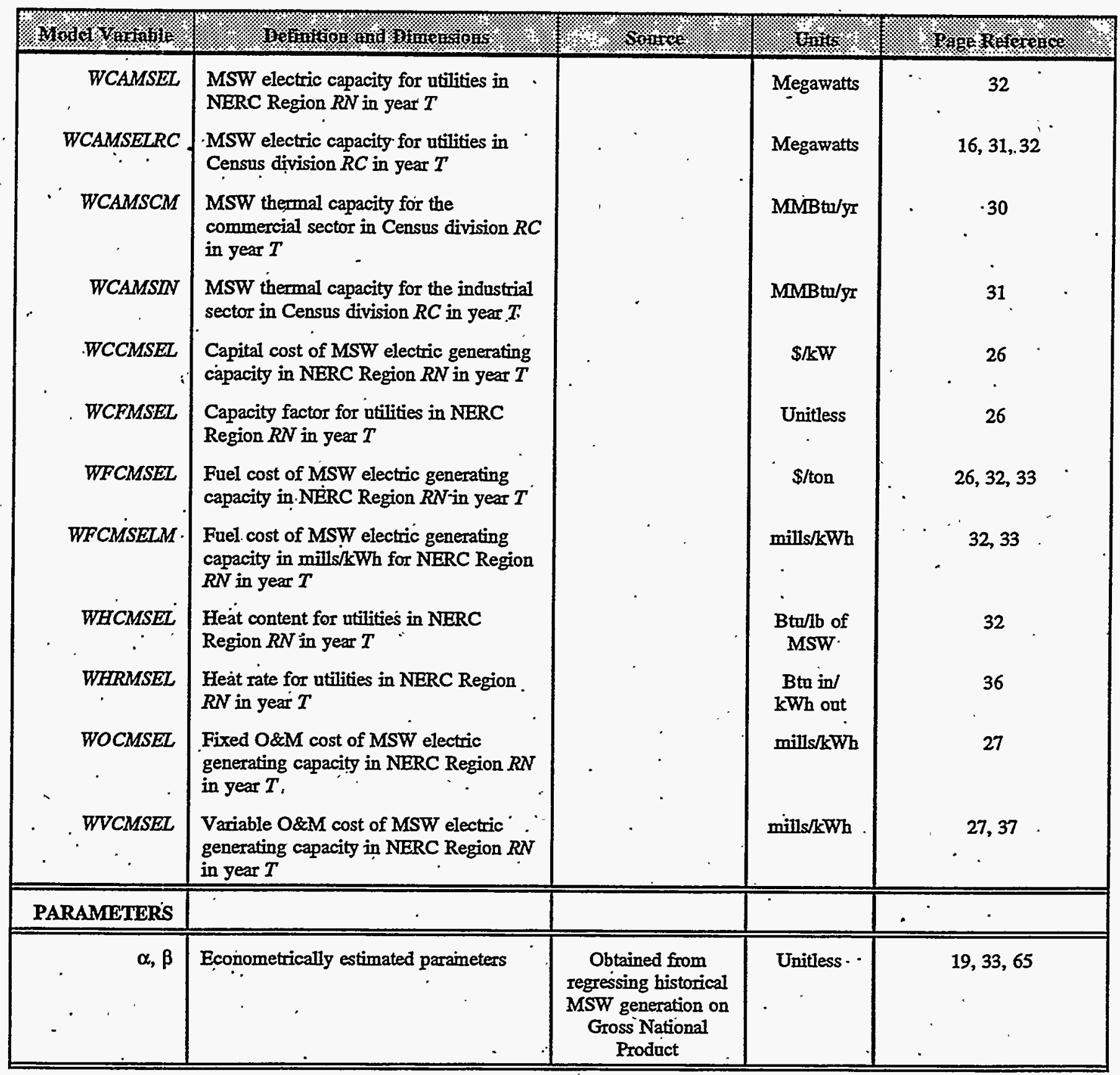

*NA $=$ Not applicable for calculated values. 


\section{MODEL INPUT: $\quad F_{\mathrm{U}, \mathrm{RC}, \mathrm{S}}$}

DEFINITION: $\quad$ Fraction of total MSW generated that is combusted for use $U$ in sector $S$ in Census Division $R C$

Once the total amount of MSW that is combusted for energy has been determined, it must be allocated among uses (electricity or other), regions (Census divisions and NERC Regions), and sectors (commercial, industrial, and utility) (Table 2A-2): The allocation factor matrix $F$ accomplishes this task by using historical and 1995 projected plant level data from the Governmental Advisory Associates (GAA) 1991 Resource Recovery Database. These data were processed with an off-line FORTRAN program that determines the values for $F$, along with the heat rate, $H R 1$, and the Census-to-NERC regional mapping factors, MPCRNR. (The FORTRAN program and the process used to derive input values for $F, H R I$, and MPCRNR are described in Appendix 2E.) The $F$ matrix used as Submodule input is provided below.

SOURCES: OakRidge National Laboratory, "Data and Sources: Biomass Supply'Draft Report," prepared for ELA under Contract No. DE-AC05-84OR21400, Oak Ridge, TN, June 27, 1993, p. 13-14.

Government Advisory Associates, Resource Recovery Yearbook and 1991 Resource Recovery Database, 177 East 87th Street; New.York, NY, 1991. 
Table 2A-2. NEMS Municipal Solid Waste Combustion Fractions

\begin{tabular}{|c|c|c|c|}
\hline . & $\begin{array}{l}\text { Electricity to the grid } \\
(U=1)\end{array}$ & $\begin{array}{l}\text { Electricity for own use } \\
(U=2)\end{array}$ & $\begin{array}{l}\text { Electricity for steam } \\
(U=3)\end{array}$ \\
\hline Commercial Sector $(S=1)$ & . & - & \\
\hline$\cdots$ & .000000 & .000000 & .001863 \\
\hline 2 & .000000 & .000000 & .005320 \\
\hline 3 & .124753 & .022944 & .000000 \\
\hline $4 \quad \cdots$ & $.000000 \cdots$ & .000000 & .029335 \\
\hline$\cdot \cdot$ & .000093 & .000103 & .006751 \\
\hline 6 & .232555 & .044410 & .000000 \\
\hline 7 & .000987 & .004572 & .017175 \\
\hline 8 & .000000 & $.000000^{\circ}$ & .015477 \\
\hline 9 & .065522 & .014119 & .000000 \\
\hline \multicolumn{4}{|l|}{ Industrial Sector $(S=2)$} \\
\hline 1 & .000000 & .000000 & .002011 \\
\hline 2 & .000000 & .000000 & .009857 \\
\hline 3 & .028898 & .005112 & .000000 \\
\hline 4 & .000000 & .000000 & .012777 \\
\hline $5:$ & .005286 & .000753 & .033947 \\
\hline 6 & .166205 & .025733 & .000000 \\
\hline 7 & .000000 & .000000 & .001103 \\
\hline$\ldots$ & .000000 & .000000 & .011644 \\
\hline 9 & $.004144 \cdot$ & .003849 & .000000 \\
\hline Utility Sector $(S .=3)$ & $\because$ & . & 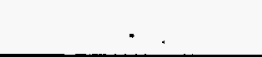 \\
\hline 1 & 000000 & .000000 & .000299 \\
\hline $2 \ldots$ & .000000 & .000000 & .002244 \\
\hline 3 & $.009209 \quad-$ & .004295 & .000000 \\
\hline 4 & .000000 & .000000 & .001955 \\
\hline $5 \cdots$ & .000000 & .000000 & .004839 \\
\hline 6 & .003451 & .000863 & .000000 \\
\hline 7 . & .000000 & .000000 & .003307 \\
\hline 8 & .000000 & .000000 & .002091 \\
\hline $9 \quad$. & .059124 & .011032 & .000000 \\
\hline
\end{tabular}


MODEL INPUT: $\quad H C$

DEFINITION: . Heat content for Census division $R C$ in year $T$.

Heat content values, measured in Btu per pound of MSW, are available in five year intervals from 1990 through 2015. The $\mathrm{HC}$ values are provided in Table 2A-3 below.

Table 2A-3. Heat Content of MSW .

\begin{tabular}{|c|c|}
\hline $\begin{array}{c}* \\
\text { Year }\end{array}$ & $\begin{array}{c}\text { Heat Content, } H C \\
\text { (Btu per pound of MSW) }\end{array}$ \\
\hline \hline 1990 & 5,114 \\
\hline 1995 & $\vdots$ \\
\hline 2000 & 5,333 \\
\hline $2005^{\circ}$ & 5,569 \\
\hline 2010 & 5,569 \\
\hline
\end{tabular}

Intermediate annual values are obtained by an interpolation subroutine named WINTERP1. Heat contents are national data, and are assumed to be the same for each Census division. The historic and projected percent composition of MSW was obtained from Franklin Associates for each of the main components of MSW. The main components of MSW include: paper and paper board; glass, metals, plastics, rubber and leather, textiles, wood, food waste, yard waste, other organics, and other inorganics. The Btu content was obtained for each material from the Office of Technology Assessment. The percentages and Btu contents were combined to provide an overall heat content per pound of MSW. Values for the years through 2000 were based on an assumed continuation of the historical increasing trend. Beyond 2000 , it was assumed that HC remains level for the duration of the forecast horizon.

SOURCES: Oak Ridge National Laboratory, "Data and Sources: Biomass Supply Draft Report," prepared for EIA under Contract No. DE-AC05-84OR21400, Oak Ridge, TN, June 27, 1993, p. 7-10.

Franklin Associates, "Characterization of Municipal Selid Waste in the United States: 1992 Update Final Report," prepared for the Environmental Protection Agency, Municipal and Industrial Solid Waste Division, Office of Solid Waste, July 1992.

Curlee, T. Randall, "Projections of Energy from the Combustion of Municipal Solid Waste: 1993 DOE-AEO Update, Draft Report, " prepared for EIA, July 1992. 
Office of Technology Assessment, Facing America's Trash: What Next for Municipal Solid Waste?, Congress of the United States, U.S. Government Printing Office, Washington, DC, October 1989.

\section{MODEL INPUT: $\quad H R I$}

\section{DEFINITION: - Heat rate for MSW energy conversion}

The heat.rate, set at $16,283.9$ Btu per kilowatthour, is assumed constant for all NERC Regions, sectors, and years. The heat rate is calculated using Governmental Advisory Associates data and an off-line FORTRAN program. For those plants that cogenerate electricity and steam, the heat rate is assumed to equal the heat rate of facilities that generate only electricity.

SOURCES: Oak Ridge National Laboratory, "Data and Sources: Biomass Supply Draft Report," prepared for EIA under Contract No. DE-AC05-84OR21400, Oak Ridge; TN, June 27, 1993, p. 15.

Government Advisory Associates, Resource Recovery Yearbook, 177 East 87th Street, New York, NY, 1991.

\section{MODEL INPUT: $\quad M P C R N R$.}

DEFINITION: - Matrix of factors for converting Census division $R C$ to NERC Region $R N$

The data used to develop conversion factors were obtained from the Government Advisory Associates (GAA) 1991 Resource Recovery Database. These conversion factors were calculated from the same FORTRAN program used to develop the $F$ matrix. The GAA data file includes WTE capacity for facilities that are expected to come on-line between 1990 and 1995. The matrix is provided in Table 2A-4.

SOURCES: Oak Ridge National Laboratory, "Data and Sources: Biomass Supply Draft Report," prepared for EIA under Contract No. DE-AC05-84OR21400, Oak Ridge, TN, June 27, 1993, p. 15.

Government Advisory Associates, Resource Recovery Yearbook and Resource Recovery Database, 177 East 87th Street, New York, NY, 1991. 
Table 2A-4. Census Division to NERC Region Conversion Factors

\begin{tabular}{|c|c|c|c|c|c|c|c|c|c|}
\hline \multirow[b]{2}{*}{$\begin{array}{l}\text { NERC } \\
\text { Region }\end{array}$} & \multicolumn{9}{|c|}{ Census Divisions } \\
\hline & 1 & 2 & 3 & 4 & 5 & 6 & 7 & 8 & 9 \\
\hline 1 & .0000 & .0466 & .6734 & .0000 & .1294 & .0000 & .0000 & .0000 & .0000 \\
\hline 2 & .0000 & .0000 & .0000 & $.0000^{\circ}$ & .0000 & .0000 & .0311 & .0000 & .0000 \\
\hline 3 & .0000 & .6003 & .0000 & .0000 & .1615 & .0000 & .0000 & .0000 & $\therefore 0000$ \\
\hline 4 & .0000 & .0000 & .3197 & .0000 & .0000 & .0000 & .0000 & .0000 & $: 0000$ \\
\hline 5 & .0092 & .0000 & .0069 & 1.0000 & .0000 & .0000 & $: 0000$ & .0000 & .0000 \\
\hline 6 & .0000 & .3530 & .0000 & .0000 & $.0000^{\circ}$ & .0000 & .0000 & .0000 & .0000 \\
\hline 7 & .9908 & .0000 & .0000 & .0000 & $\because 0000$ & .0000 & .0000 & .0000 & .0000 \\
\hline 8 & .0000 & .0000 & .0000 & .0000 & .6072 & .0000 & .0000 & .0000 & .0000 \\
\hline 9 & .0000 & .0000 & .0000 & .0000 & .1018 & 1.0000 & .0000 & .0000 & .0000 \\
\hline 10 & .0000 & .0000 & .0000 & $.0000^{\circ}$ & .0000 & .0000 & $.9689^{\circ}$ & .0000 & .0000 \\
\hline 11 & .0000 & .0000 & .0000 & .0000 & .0000 & .0000 & .0000 & 1.0000 & .4088 \\
\hline 12 & .0000 & .0000 & .0000 & .0000 & .0000 & .0000 & .0000 & .0000 & .0000 \\
\hline 13 & .0000 & .0000 & .0000 & .0000 & .0000 & .0000 & .0000 & $.0000^{\circ}$ & .5912 \\
\hline
\end{tabular}

\section{MODEL INPUT: $\quad P C C$}

\section{DEFINITION: $\quad$ Percent MSW combusted for Census division $R C$ in year $T$}

National percent combusted values, available in 5-year intervals, are presented in Table $2 \mathrm{~A}-5$.

These percentage values are divided by 100 to produce factors. The MSW Submodule then calculates intermediate annual values using an interpolation subroutine named WINTERP1. Finally, the PCC factors are read into a regional PCC matrix; thereby yielding $P C C$ values that are constant across all Census divisions.

Estimates of percent of MSW combusted for 1.960 through 1990 were obtained from Franklin Associates. Data for the years after 1990 are projections based on analyses conducted by the Oak Ridge National Laboratory and EIA staff. 
Table 2A-5. Percent MSW Combusted

\begin{tabular}{|c|c|}
\hline Year & $\begin{array}{c}\text { Percent MSW } \\
\text { Combusted, } \\
\text { PCC }\end{array}$ \\
\hline \hline 1990 & 15.2 \\
\hline 1995 & 20.0 \\
\hline 2000 & 25.0 \\
\hline 2005 & 28.0 \\
\hline 2010 & $\ddots 30.0$ \\
\hline
\end{tabular}

SOURCES: OakRidge National Laboratory, "Data and Sources: Biomass Supply Draft Report," prepared for ELA under Contract No. DE-AC05-84OR21400, Oak Ridge, TN, June 27, 1993, p. 12.

Franklin Associates, "Characterization of Municipal Solid Waste in the United States: 1992 Update Final Report," prepared for the Environmental Protection Agency, Municipal and Industrial Solid Waste Division, Office of Solid Waste, July 1992.

Office of Technology Assessment, Facing America's Trash: What Next for Municipal Solid Waste?, Congress of the United States, U.S. Government Printing Office, Washington, DC, October 1989.

Curlee, T. Randall, "MSW Projections for the EIA 1992 Annual Energy Outlook, Draft Report," prepared for EIA, August 1991.

\section{MODEL INPUT: RCAPFEL}

\section{DEFINITION: : Capacity fáctor for a Waste-to-Energy (WTE) plant}

The capacity factor is obtained from the EPRI Technical Assessment Guide (TAG). It is assumed to be 0.85 for all regions and years. The MSW submodule assigns this capacity factor value to the RFM common block variable, WCFMSEL.

SOURCES: Oak Ridge National Laboratory,. "Data and Sources: Biomass Supply Draft Report," prepared for EIA under Contract No. DE-ACC5-84OR21400,-Oak Ridge, TN, June 27, 1993, p. 19.

Electric Power Research Institute, TAG Technical Assessment Guide. EPRI P-6587L, .Vol. 1: Rev. 6, Palo Alto, CA, 1989. 
MODEL INPUT: $\quad R C A P$

\section{DEFINITION: Capital cost of a WTE plant}

The unadjusted capital cost, $\$ 4,630$ per kilowatt, is obtained from the EPRI Technical Assessment Guide (TAG). The TAG capital cost is expressed in December 1988 dollars, and is converted to 1987 dollars by multiplying the cost by the ratio of the Implicit GNP Price Deflator value midway between the 1988 and 1989 values, and the 1987 Deflator value. The adjusted capital cost value used as model input is $\$ 4,389$ per kilowatt. The MSW submodule assigns this capital cost value to the RFM common block variable, WCCMSEL.

SOURCES: Oak Ridge National Laboratory, "Data and Sources: Biomass Supply Draft Report," prepared for EIA under Contract No. DE-AC05-84OR21400, Oak Ridge, TN, June 27, 1993, p. 19.

Electric Power Research Institute, TAG Technical Assessment Guide. EPRI P-6587L, Vol. 1: Rev: 6, Palo Alto, CA, 1989.

\section{MODEL INPUT: RNCFUEL}

\section{DEFINITION: Tipping fee charged for MSW.}

The tipping fee is structured as a negative adjustment to the fuel cost variable, WFCMSEL. Tipping fees were calculated based on data from Chupka, Howarth, and Zoi. The tipping fees, originally expressed in dollars per ton of MSW, are aggregated to Census Regions using MSW facility consumption.weighting factors, converted to real 1987 dollars, and then transformed into mills-per-kilowatthour.

SOURCES: ' Oak Ridge National Laboratory, "Data and Sources: Biomass Supply Draft Report;" prepared for EIA under Contract No. DE-AC05-84OR21400, Oak Ridge, TN, June 27, 1993, p. 18.

Chupka, Marc, D. Howarth, and C. Zoi. Renewable Electric Generation: An. Assessment of Air Pollution Prevention: Potential. EPA/400/R-92/005, U.S. Environmental Protection Agency, 1992.

\section{MODEL' INPUT: $\quad R C O M$}

DEFINITION: Fixed operation \& maintenance (O\&M) cost for a WTE plant

Data for calculating operating costs are obtained from the EPRI Technical Assessment Guide (TAG). Data are available for mass burn technology and refuse derived fuel. Information for the mass burn technology is used in the calculations. Fixed operating and maintenance costs are listed 
" as $\$ 118.6$ per kilowatt per year. At an 85 percent capacity factor, this equals $\$ 0.0159$ per $\mathrm{kWh}$ in December 1988 dollars. To convert from December 1988 dollars to 1987 dollars, the O\&M cost is multiplied by the ratio of the Implicit GNP Price Deflator value midway between the 1988 and 1989 values, and the 1987 Deflator value. Therefore, in real 1987 dollars, the fixed O\&M cost is $\$ 0.0151$ per $\mathrm{kWh}$, or 15.1 mills per $\mathrm{kWh}$. The MSW submodule assigns this O\&M cost value to the RFM common block variable, WOCMSEL.

SOURCES: Oak Ridge National Laboratory, "Data and Sources: Biomass Supply Draft Report,". prepared for EIA under Contract No. DE-AC05-84OR21400, Oak Ridge, TN, June 27, 1993, p. 19.

Electric Power Research Institute, TAG Technical Assessment Guide. EPRI P-6587L, Vol. 1: Rev. 6, Palo Alto, CA, 1989.

\section{MODEL INPUT: RVOM}

\section{DEFINITION: . Variable O\&M cost for a WTE plant}

Data for calculating the operating cost are obtained from the EPRI Technical Assessment Guide (TAG). Data are available for mass burn technology and refuse derived fuel. Information for the mass burn technology is used in the calculations. Variable operating costs are listed as 0.0181 $\$ / \mathrm{kWh}$ in 1988 dollars. To convert from December 1988 dollars to 1987 dollars, the O\&M cost is multiplied by the ratio of the Implicit GNP Price.Deflator value midway between the 1988 and 1989 values, and the 1987 Deflator value. Therefore, in real 1987 dollars, the variable O\&M cost is $0.0173 \$ / \mathrm{kWh}$, or 17.3 mills per. $\mathrm{kWh}$. Finally, the variable operating cost is adjusted by subtracting the tipping fee, and assigning the operating cost value to the RFM common block .variable, WVCMSEL.

SOURCE: Oaki Ridge National Laboratory, "Data and Sources: Biomass Supply Draft-Report," prepared for EIA under Contract No. DE-AC05-84OR21400, Oak Ridge, TN, June 27, 1993, p. 19.

MODEL INPUT: SR

DEFINITION: Annual source reduction factor

The annual source reduction factor, based on expert judgement from Oak Ridge National Laboratory is 0.5 percent per year, or 0.005 . For additional details on the derivation of $S R$, see Appendix 2E.

SOÜRCES: Oak Ridge National Laboratory, "Data and Sources: Biomass Supply Draft Report," prepared for EIA under Contract No. DE-AC05-84OR21400, Oak Ridge, TN, June 27,1993, p. 6. 



\section{Appendix 2-B: Mathematical Description}

This Appendix provides the detailed mathematical specification of the MSW Submodule as presented in the RFM FORTRAN code execution sequence. Subscript definitions are also as they appear in the. FORTRAN code.

The generation of MSW is postulated to be primarily a function of gross domestic product (GDP) and population. Empirical analysis shows that only GDP is necessary for a regression with a high coefficient of determination $\left(R^{2}\right)$. Therefore, a baseline amount of MSW generated nationally is computed in Equation 2B-1. This amount if further adjusted in the model in a manner discussed later. Therefore:

$$
M S W=\alpha+\beta x M C_{-} G D P_{T}
$$

where:

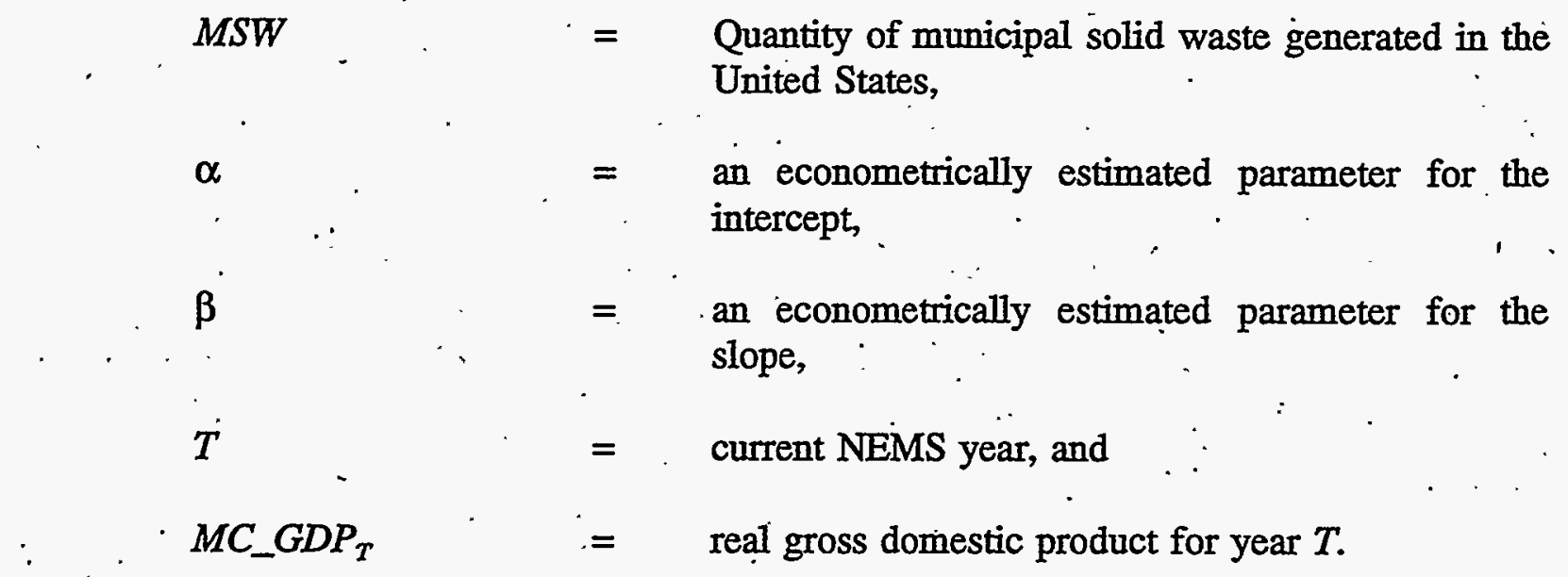

The Analysis of Variance, with MSW as the dependent variable, yielded the following:

\begin{tabular}{|c|c|c|c|c|c|}
\hline Parameter & $\underline{\mathrm{DF}}$ & $\begin{array}{l}\text { Parameter } \\
\text { Estimate }\end{array}$ & $\begin{array}{l}\text { Standard } \\
\text { Error }\end{array}$ & $\begin{array}{l}\text { Probability } \\
\geq \mid \mathbf{T}\rfloor\end{array}$ & $\begin{array}{l}\mathbf{T} \text { for HO } \\
\text { Param. }=0 .\end{array}$ \\
\hline$\hat{\boldsymbol{\alpha}}$ & 1 & 14.60056435 & 5.33926732 & 0.0411 & 2.735 \\
\hline$\widehat{\mathcal{B}}$ & 1 & 0.03599054 & 0.001522472 & 0.0001 & 23.640 \\
\hline
\end{tabular}

Durbin-Watson D - 2.501.

Number of Observations - 7 


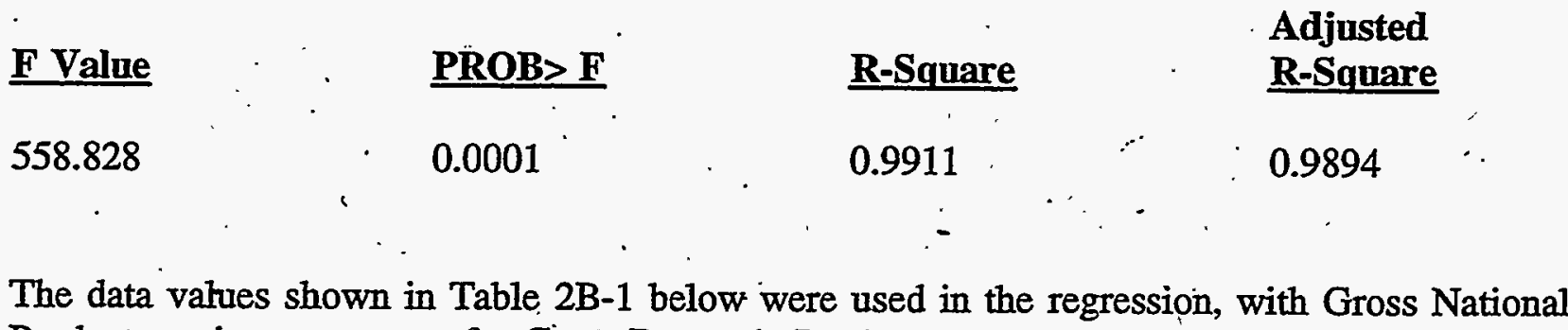
Product serving as a proxy for Grosis Domestic Product:

Table 2B-1: Historical MSW Quantity and GNP Values

\begin{tabular}{|c|rr|rr||}
\hline Year & \multicolumn{2}{|c|}{$\begin{array}{c}\text { Historical MSW Quantity } \\
\text { (Million of Tons) }\end{array}$} & \multicolumn{2}{c|}{$\begin{array}{c}\text { Gross National Product } \\
\text { (Billions of 1987 Dollars) }\end{array}$} \\
\hline \hline 1960 & & 87.8 & & 1985.1 \\
\hline $1965:$ & & 103.4 & & 2491.9 \\
\hline 1970 & & 121.9 & & 2893.5 \\
\hline 1975 & & 128.1 & & 3247.6 \\
\hline 1980 & & 151.4 & & 3823.4 \\
\hline 1985 & & 164.4 & & 4295.0 \\
\hline 1990 & & 195.7 & & 4894.6 \\
\hline
\end{tabular}

The adjusted $R^{2}$ of 0.9894 shows a high degree of correlation.

The model incorporates an additional factor for source reduction, discussed below, into the equation for waste generation.

\section{Source Reduction Factor}

Projections of MSW generation quantities based on the above regression approach must be modified because of structural market changes that are occurring and are likely to occur in future years. Governments and businesses have adopted strategies to lessen the amount of waste generated without reducing economic output. The general term for these strategies is source reduction. An example of such a strategy is the local government trend toward unit-based disposal rates, which has brought about a reduction of generated waste where implemented. Also, as of 1992 at least 38 States have passed laws mandating that disposal of their municipal waste 
streams be reduced by 25 percent. or more by no later than the year $20000^{5}$ Such goals can be met through a combination of source reduction and recycling. To the extent that source reduction strategies are successful, they will likely alter the basic relationship between GDP and MSW quantity.

In order to reflect anticipated annual reductions in the quantity of MSW generated on account of source reduction efforts, the quantity projected by the MSW supply equation will be reduced by an exogenously-determined source reduction multiplier. This multiplier, $S R$, will be based in part on legislation passed or proposed to promote source reduction. Currently, EIA uses expert judgement to derive the $S R$ parameter of 0.005 that is currently used in the MSW supply equation.

The quantity of waste generation, QNAT, is therefore represented as follows:

$$
Q N A T=M S W x[1-(T-1) x S R]
$$

where:

$\begin{array}{lll}\text { QNAT } & = & \text { Projected MSW generation in the United States, } \\ T & = & \text { current NEMS year, and } \\ S R & = & \text { annual source reduction factor. }\end{array}$

Within the MSW Submodule, Equations $2 \mathrm{~B}-1$ and $2 \mathrm{~B}-2$ are combined into a single equation:

$$
Q N A T=\alpha+\beta x M C_{-} G D P_{T} x[1-(T-1) x S R]
$$

\section{Fraction of MSW Combusted}

The ORNL baseline MSW combusted fractions used in the MSW Submodule reflect an optimistic outlook for long-term market penetration of WTE facilities. This outlook is based on five major expected trends pointing toward increased reliance on WTE facilities as waste management options. First, the number of landfills is dropping rapidly and will continue to do so as recent Resource Conservation and Recovery Act (RCRA) landfill rules are put in place. Further, the cost of landfilling is likely to increase significantly both in absolute terms-as well as relative to the cost of building and operating WTE facilities. Second, barriers to the financing of WTE facilities that resulted primarily from the Tax Reform Act of 1986 are likely to be overcome gradually. These financing barriers have played a role in decisions to cancel several WTE facilities. Third, the environmental effects of. WTE operations continue to be assessed, and the opposition to WTE may subside somewhat as the public becomes more informed about the environmental

${ }^{5}$ Glenn, J., "The State of Garbage in America," Biocycle, May 1992, pp. 30-37. 
consequences of WTE relative to other waste management options. Fourth, there is increasing speculation that the recycling goals of 25 to 50 percent mandated or targeted in many States will not.be reached. If this speculation is correct, communities will turn increasingly to WTE as the preferred alternative. Finally, it is likely that the U.S. Congress will grant States limited authority to restrict the importation of MSW into their States for disposal. Recent attempts by States to restrict the importation of waste have thus far been overturned in the Court's on the grounds that such bans violate the interstate commerce provisions of the U:S. Constitution. However, if Congress allows States to limit MSW importation, many populous States will be faced with the increased burden of managing their own waste. In this event, WTE is likely to receive greater attention as a waste management option.

Because of these factors, ORNL expects that WTE will claim an increasing share of MSW management, especially beyond the year 2000 .

ORNL's percent-combusted values were then adjusted using a regional estimation procedure applied by EIA staff. First; the Franklin Associates/EPA value for total waste (195.7 million tons per year) was proportionally disaggregated into Census divisions according to the populations in each Census division. This disaggregation assumes a constant per-capita MSW generation rate for all regions because there is no known data on regional MSW generation or rates.

The existing facility design capacity, in tons per day, is then identified and aggregated into Census divisions using information from Waste Age magazine. ${ }^{6}$ A value for tons of MSW combusted is estimated by applying a utilization factor of. 0.90 , which is consistent with the value derived from the EPA data. The estimated combusted-shares in each Census division is obtained by dividing the tons-combusted values by the total waste generated in Census division.

The regional combusted-shares for 2010 were established based on the following rules:

1. No regional share will be above 50 percent. Thus, combusted-share values for two regions (New England and Alaska-Hawaii) decline to 50 percent. This rule is based on the assumption. of increased recycling, which is expected to grow to 40 percent in many jurisdictions. At least 10 percent is expected to be landfilled in any case since some material cannot be recycled or burned.

2. The Mid-Atlantic Census Division is assumed to be limited to a combusted-share of 40 percent, since those States have more robust,recycling goals.

3. Combusted-shares for all other regions are assumed to increase at an annual rate of 5 percent from 1990 through 2010.

The average of the resulting values, weighted by waste generated, is about 30 percent for 2010; which is used as the national value. Use of regional shares directly would require that these values be derived by NERC regions, and data is not readily available for such a calculation.

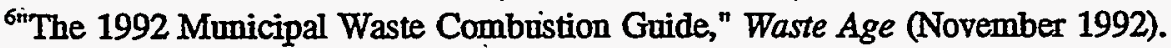


Data used to derive the adjusted combusted-shares and results are provided in Table 2B-2. The intermediate combustion shares are linearly interpolated between 1990 and 2010.

Table 2B-2. Regional Estimates of MSW Combustion Shares, 1990 and 2010

\begin{tabular}{|c|c|c|c|c|c|}
\hline$\because$ & & & & $\begin{array}{r}\text { Percent } \mathrm{C} \\
\text { She }\end{array}$ & busted \\
\hline Census Division & $\begin{array}{c}1990 \\
\text { Population }\end{array}$ & $\begin{array}{l}\text { Waste } \\
\text { Generation } \\
\text { (Tons per } \\
\text { Day) }\end{array}$ & $\begin{array}{l}\text { Combustion } \\
\text { Capacity } \\
\text { (Tons per } \\
\text { Day) }\end{array}$ & 1990 & 2010 \\
\hline New England & $13,206,943$ & $.28,471.3$ & 17,860 & 0.56 & 0.50 \\
\hline Mid Atlantic & $37,602,286$ & $81,062.2$ & 15,626 & 0.17 & 0.40 \\
\hline East North Central & $42,008,942$ & $90,562.0$ & 13,827 & 0.14 & 0.36 \\
\hline West North Central & 18,$867 ; 612$ & $40,674.0$ & 5,743 & 0.13 & 0.34 \\
\hline South Atlantic & $42,359,131$ & $91,316.9$ & 25,416 & 0.25 & 0.50 \\
\hline East South Central & $15,176,284$ & 32716.7 & 2,770 & 0.08 & 0.20 \\
\hline West South Central & $26,702,793$ & $57,565.3$ & 1,773 & 0.03 & 0.07 \\
\hline Mountain & $13,658,776$ & $29,445.3$ & 522 & 0.02 & 0.04 \\
\hline Pacific & $7,709,013$ & $16,618: 9$ & 2,560 & 0.14 & 0.37 \\
\hline California & $29,760,021$ & $64,156.0$ & 1,328 & 0.02 & 0.05 \\
\hline Alaska-Hawaii & $1,658,632$ & $3,575.6$ & 2,205 & 0.56 & 0.50 \\
\hline U.S. TOTAL & $248,710,233$ & $536,164.4$ & 89,630 & 0.15 & 0.30 \\
\hline
\end{tabular}


- Equation 2B-4 determines the quantity shares of energy produced from MSW:

$$
Q_{U, R C, S}=Q N A T \times P C C_{R C, T} \times H C_{R C, T} \times F_{U, R C, S} \times 2000
$$

where:

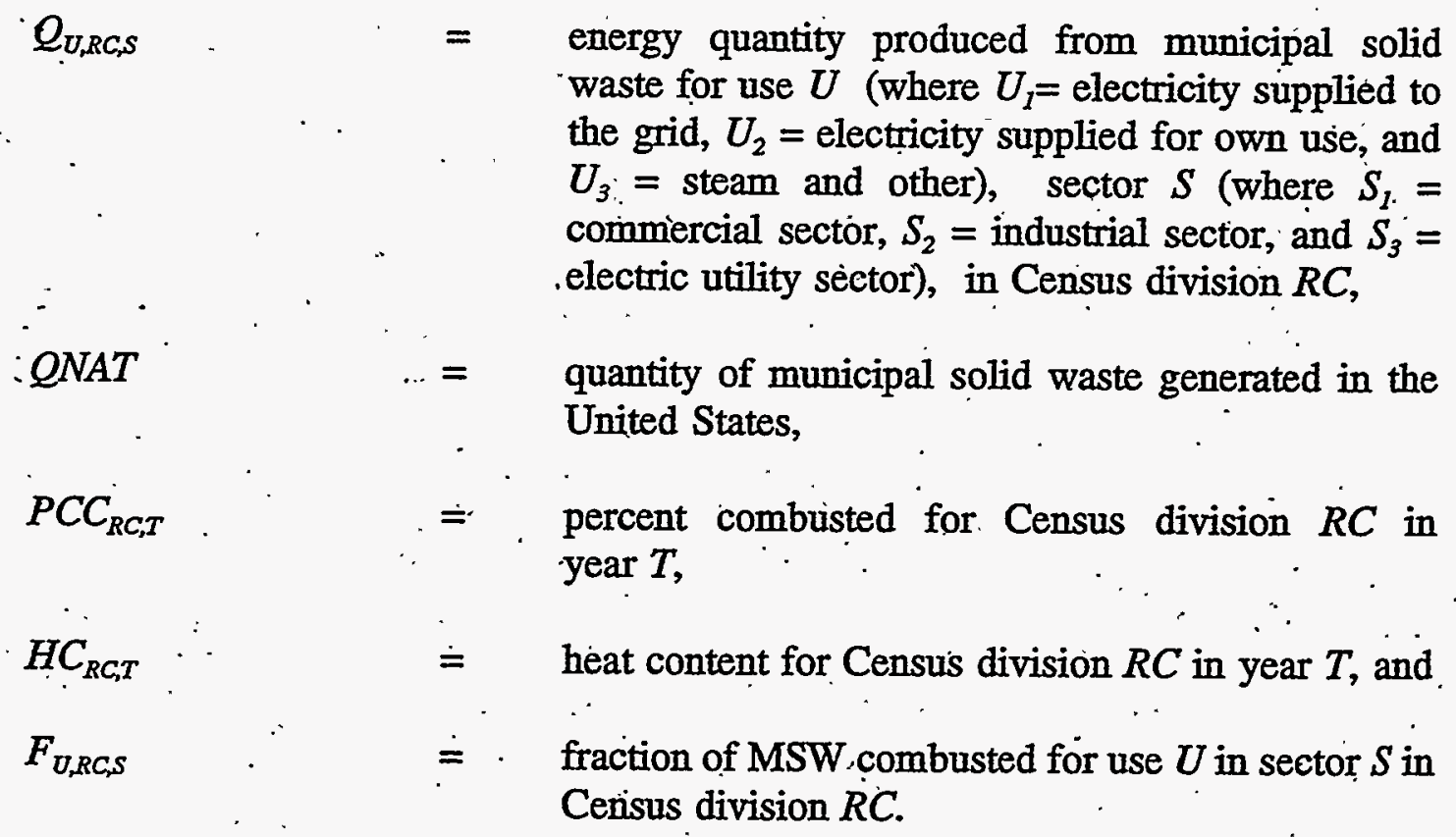

Equations $2 \mathrm{~B}-5$ and $2 \mathrm{~B}-6$ combine the production capacity for all energy forms (electricity and other) into a total energy production capacity for commercial and industrial sectors:

$$
W C A M S C M_{R C, T}=\sum_{U=1}^{3} Q_{U, R C, S=1}
$$

where:

$$
\begin{array}{ccc}
W_{C A M S C M_{R C, T}}= & \begin{array}{l}
\text { MSW energy production capacity in millions of Btu } \\
\text { for the commercial sector by Census division } R C \text { in } \\
\text { year } T, \text { and, }
\end{array} \\
Q_{U, R C S=1} & =\begin{array}{l}
\text { MSW energy quantity produced from municipal } \\
\text { solid waste for use } U \text { (where } U_{1}=\text { electricity }
\end{array} \\
& \therefore \quad \begin{array}{l}
\text { supplied to the grid, } U_{2}=\text { electricity supplied for } \\
\text { own use, and } U_{3}=\text { steam and other), in the } \\
\text { commercial sector }(S=1) \text { in Census division } R C .
\end{array}
\end{array}
$$

where: 


$$
W_{C A M S I N}=\sum_{U=1}^{3} \dot{Q}_{U, R C, S=2}
$$

$$
\begin{aligned}
& W_{C A M S I N} N_{R C T} \cdots=\text { MSW energy production-capacity in millions of Btu } \\
& \text { for the industrial sector by Census division } R C \text { in } \\
& \text { year } T \text {, and, } \\
& Q_{U, R C S=2} \quad=\quad \mathrm{MSW} \text { energy quantity produced from municipal } \\
& \text { solid waste for use } U \text { in the industrial sector ( } \mathrm{S}=2 \text { ) } \\
& \text { by Census division } R C \text {. }
\end{aligned}
$$

\begin{tabular}{|c|c|c|}
\hline $\begin{array}{c}\text { WCAMSELRC } \\
:\end{array}$ & $=$ & $\begin{array}{l}\text { Annual MSW electric capacity in megawatts for } \\
\text { own-use. and sale to the utility grid in Census } \\
\text { division } R C \text { in year } T \text {, }\end{array}$ \\
\hline & $\therefore$ & Heat rate for MSW (Btu in/kWh out), and, \\
\hline$Q_{U, R C, S=3}$ & $=$ & $\begin{array}{l}\text { Annual electricity production from } M S W \text { in millio } \\
\text { of Btiv for both sale to the grid }(U=1) \text { and own- } \\
(\mathrm{U}=2) \text {, in the electric utility sector }(S=3) \text {, in Cens } \\
\text { division } R C \text {. }\end{array}$ \\
\hline
\end{tabular}

'Equation 2B-7 calculates the annual electric capacity available for WTE facility own-use and sale to the electric utility grid:

$$
\text { WCAMSELRC } C_{R C, T}=\frac{\left[\sum_{U=1}^{2} Q_{U, \dot{R} C, S=3}\right] \times 10^{6}}{H R I \times 8760 \times 1000}
$$

where: 
Equation 2B-8 maps annual electric capacity from Census divisions to NERC regions:

$$
\text { WCAMSEL }_{R N, T}=\sum_{R C=1}^{9} W C A M S E L R C_{R C, T} x M P C R N R_{R C, R N}
$$

where:

$$
\begin{array}{lll}
W C A M S E L_{R N, T} & = & \begin{array}{l}
\text { Electric capacity in megawatts in NERC Region } \dot{R} N \\
\text { in year } T,
\end{array} \\
W C A M S E L R C_{R G T} & = & \begin{array}{l}
\text { Electric capacity in megawatts for Census division } \\
R C \text { in year } T, \text { and }
\end{array} \\
M P C R N R_{R C, R N} & = & \begin{array}{l}
\text { Conversion factor matrix for mapping Census } \\
\text { division } R C \text { to NERC Region } R N .
\end{array}
\end{array}
$$

\begin{tabular}{|c|c|c|}
\hline$W F C M S E L M_{R N, T}$ & $=$ & $\begin{array}{l}\text { Fuel cost in mills/kWh for a WTE plant for NERC } \\
\text { Region } R N \text { in year } T \text {, }\end{array}$ \\
\hline$W F C M S E L_{R N, T}$ & $=$ & $\begin{array}{l}\text { Fuel cost (tipping fee) in dollars per ton for a WTE } \\
\text { plant for NERC Region } R N \text { in year } T \text {, }\end{array}$ \\
\hline$W H C M S E L_{R N, T}$ & $=$ & $\begin{array}{l}\text { Heat content of MSW in NERC Region } R N \text { for year } \\
T,\end{array}$ \\
\hline 2000 & $=$ & Number of pounds in a ton, and \\
\hline WHRMSEL $L_{R N, T}$ & $=$ & $\begin{array}{l}\text { Heat rate for utilities in NERC Region } R N \text { in year } \\
T \text {. }\end{array}$ \\
\hline
\end{tabular}

Equation 2B-9 transforms the fuel cost (tipping fee) from dollars-per-ton units to mills-perkilowatthour units:

$$
\text { WFCMSELM }_{R N, T}=\frac{\dot{W F C M S E L_{R N, T}} \times \text { WHRMSEL } L_{R N, T} \times 1000}{W H C M S E L_{R N, T} \times 2000}
$$

where:

Equation 2B-10 calculates WTE facility variable operating costs: 


$$
W_{W C M S E L_{R N, T}}=R V O M-W F C M S E L M
$$

where:

\begin{tabular}{|c|c|c|}
\hline$W V C M S E L_{R N, T}$ & $=$ & $\begin{array}{l}\text { RFM variable operating cost common block variable } \\
\text { for WTE facilities in NERC Region } R N \text { in year } T \text {, }\end{array}$ \\
\hline$R V O M$ & $=$ & $\begin{array}{l}\text { Variable operation \& maintenance cost in mills } \\
\text { kilowatthour, }\end{array}$ \\
\hline$M S E L M_{R N, T}$ & $=$ & $\begin{array}{l}\text { Fuel cost (tipping fee) in mills/kWh for a WTE } \\
\text { plant for NERC Region } R N \text { in year } T \text {, }\end{array}$ \\
\hline
\end{tabular}




\section{Appendix 2-C: Bibliography}

Chupka, Marc, D. Howarth, and C. Zoi. Renewable Electric Generation: An Assessment of Air Pollution Prevention Potential. EPA/400/R-92/005, U.S. Environmental Protection Agency, 1992.

Curlee, T. Randall, "MSW Projections for the EIA 1992 Annual Energy Outlook, draft report," Oak Ridge National Laboratory, prepared for the Enèrgy Information Administration, U.S. Department of Ėnergy, August 1991.

Curlee, T. Randall; "Projections of Energy from the Combustion of Municipal Solid Waste: 1993 DOE-AEO Update," draft report, prepared for the Energy Information Administration, U.S. Department of Energy, July 1992.

Electric Power Research Institute, TAG Technical Assessment Guide. EPRI P-6587-L, Vol. 1: Rev. 6; Palo Aïto, CA, 1989.

Environmental Protection Agency, Compilation of Air Pollutant.Emission Factors, Vol. 2. Stationary Point and Area Sources, 4th edition. Supplement D, AP-42, September 1991, p. 2.1-7.

Franklin Associates, "Characterization of Municipal Solid Waste in the United States: 1992 Update Final Report," prepared for the Environmental Protection Agency, Municipal and Industrial Solid Waste Division, Office of Solid Waste, July 1992.

Glenn, Jim, "The Ștate of Garbage in America," BioCycle 33(5): pp. 30-37, May $1992 .$.

Government Advisory Associates, Resource Recovery Yearbook, 177 East 87th Street, New York, NY, 1991.

National Solid Waste Management Association, "The'1992 Municipal Waste Combustion Guide," . Waste Age 23(11): pp. 99-117, November 1992.

Oak Ridge National Laboratory, "Data and Sources: Biomass Supply Draft Report," prepared for EIA under Contract No. DE-AC05-84,OR21400, Oak Ridge, TN, June 27, 1993, p. 1314.

Office of Technology Assessment, Facing America's Trash: . What Next for Municipal Solid Waste?, Congress of the United States, U.S. Government Printing Office, Washington, DC, October 1989. 


\section{Appendix 2-D: Model Abstract}

\section{Model Name:}

Municipal Solid Waste Submodule

\section{Modèl Acronym:}

MSW

\section{Description:}

The submodule uses the quantity of municipal solid waste produced (derived econometrically), the heating value of MSW, and forecasted shares of MSW combusted for energy recovery to produce forecasts of the production of electricity and other energy forms (steam and direct heat). Forecasts are disaggregated by consuming sector (commercial, industrial, and electric utility) and region. .

\section{Purpose of the Model:}

The MSW Submodule provides the NEMS commercial and industrial sector modules with annual regional projections of energy produced from the incineration of municipal solid waste. For the NEMS Electricity Market Module, the submodule provides regional forecasts of electric capacity to be decremented from electric utility capacity requirements, as well as capital and operating costs for the calculation of electricity prices.

\section{Most Recent Model Update:}

December 1993

\section{Part of Another Model?: :}

The MSW submodule is a component of the Renewable. Fuels Module (RFM) of the National Energy'Modeling System (NEMS).

\section{Official Model Representative:}

Roger Diedrich

Coal, Uranium, and Renewable Fuels Analysis Branch

Energy Information Administration

Phone: . (202) 586-0829

\section{Documentation:}

Model Documentation Report: Reriewable Fuels Module, March 1994.

\section{Archive Media and Installation Manual(s):}

Archived as part of the NEMS production runs.

\section{Enèrgy System Described:}

Byproduct energy production and consumption from the combustion of municipal solid waste. 


\section{Coverage:}

- Geographic: Nine Census Divisions-New England, Mid-Atlantic, East North Central; West North Central, South Atlantic, East South Central, West South Central, Mountain, Pacific

- Time Unit/Frequency; Annual, 1990 through 2015

- . Products: electricity, steam

- Economic Sectors: 'commercial sector, industrial' sector, electric utility sector

\section{Modeling Features:}

- Model Structure: Sequential calculation of forecasted national municipal solid waste (MSW) generation; followed by derivation of regional and sector energy shares based on estimates of the percentage of MSW combusted.

- Modeling Technique: Econometric estimation of municipal solid waste generation, coupled with an energy share allocation algorithm for deriving electric generation capacity and energy quantities by sector and region.

- Special Features: Allows for the modeling of regional and national resource recovery efforts

\section{Non-DOE Input Sources:}

Franklin Associates, data prepared for the Environmental Protection Agency:

- National annual quantity of municipal solid waste generated

Forecasted annual percentages of municipal solid waste combusted

Government Ádvisory Associates, Resource Recovery Yeäbook and Resource Recovery Database:

- Plant-specific electricity generation, Btu energy content of MSW

- Plant locations and energy consuming sectors

Electric Power Research Institute, TAG Technical Assessment Guide:

- Capital cost; fixed and váriable operation \& maintenance costs

- Plant capacity factor 
DOE Input Sources:

None.

Computing Environment:

- Hardware Used: IBM 3090

- Operating System: MVS

- Language/Software used: VS FORTRAN, Yer. 2.05

- Memory Requirement: $11 \mathrm{~Kb}$

- Storage Requirement: $6 \mathrm{~Kb}$ tracks of an IBM 3380 disk pack

- Estimated Run Time: 0.02 seconds

- Special Features: None

Independent Expert Reviews Conducted:

None.

Status of Evaluation Efforts by Sponsor:

None.

Energy Information Administration/NEMS Renewable Fuels Module Documentation Report-MSW

43 



\section{Appendix 2-E: Data Quality and Estimation Processes.}

This Appendix discusses (1) the quality of the principal sources of input data used in the MSW Submodule, along with a discussion of user-defined parameters and guidelines used to select them, and (2) estimation methods used to derive parameters.

\section{Governmental Advisory Associates Resource Recovery Database}

The original GAA data file consists of 20 columns of data items and 202 rows corresponding to each MSW facility in the GAA database. The 20 data items include the following: plant number, status, start month, start year, planned start month, planned start year, shutdown date, State, capacity, annual throughput of MSW, output type, gross capacity (2MW), net capacity (MW), gross $\mathrm{kWh} / \mathrm{ton}$, net $\mathrm{kWh} / \mathrm{ton}, \mathrm{Btu} / \mathrm{lb}$, Census division, NERC Region, sector, and a consuming 'sector code. Census division was determined by the State, and NERC Region was determined from a-map of NERC Regions and the location of the facilities in the GAA data. Allocation by sector was determined according to the primary customer, not ownership of the facility. Military installations and sewage and water treatment facilities were assumed to be part of the industrial sector. Universities, prisons, police stations, and district heating facilities were included in the commercial sector. There was considerable missing data in the GAA database, particularly for electricity generation and energy content. Data were filled in using averages across W:TE facilities using the following procedures:

1. If there is no net $\mathrm{kWh} / \mathrm{ton}$, but there are data on gross $\mathrm{kWh} / \mathrm{ton}$, then use the following equation:

$$
\text { netkWh/ton }=\text { grosskWh/ton } \dot{x} \frac{\text { net } M W}{\text { gross } M W}
$$

2. If there is no gross $\mathrm{kWh} / \mathrm{ton}$, but there are data on net $\mathrm{kWh} / \mathrm{ton}$, then use the following equation:

$$
\text { grosskWh/ton }=\text { netkWh/ton } \times \frac{\operatorname{gross} M W}{\text { net } M W}
$$


3. If there is no net capacity, but there are data on gross capacity, then use the following:

$$
\text { net } M W=\text { gross } M W \times \frac{\text { average } \text { net } M W:}{\text { average gross } M W}
$$

Averages were determined for all plants generating electricity however, averages differentiated between those plants that generate only electricity and those cogenerating electricity and steam.

4. If there is no net capacity or gross capacity, the following two equations were used.

$$
\begin{gathered}
\text { net } M W:=\text { annual throughput of } M S W \times \frac{\text { average net } M W}{\text { average throughput }} \\
\text { gross } M W=\text { annual throughput of } M S W \times \frac{\text { average gross } M W}{\text { average throughput }}
\end{gathered}
$$

Averages differentiate between electricity only and cogeneration. facilities.

5. If there is no net $\mathrm{kWh} /$ ton or gross $\mathrm{kWh} / \mathrm{ton}$, then use the following two equations.

$$
\begin{aligned}
& \text { grosskWh/ton }=\text { average grosskWh/ton } \\
& \text { netkWh/ton }=\text { grosskWh/ton } x \frac{\text { net } M W}{\text { gross } M W}
\end{aligned}
$$

6. If there is no heat content (Btu/lb), then use the following equation.

$$
\text { Btullb = average Btullb }
$$

In this case, the average heat content is computed across all facilities regardless of the output.

The GAA data file was then reduced to include only those data items necessary for calculating $F$, HR, and MPCRNR. This reduced data file is used as input to the FORTRAN program that determinies $F ; H R$, and MPCRNR: 
The raw data from the GAA database, with no missing values filled in, are shown in Table $2 \mathrm{E}-1$. This table also shows the sector codes indicating the end-use sector to which the energy is sold. ${ }^{7}$ Table 2E-2 shows the data with missing data filled in. In this table, the WTE plants are sorted by output type and Census region within output type. The column names for both tables are as follows: $1=$ plant number, $2=$ status, 3 =start month, $4=$ start year, $5=$ planned start month, $6=$ planned start year, $7=$ month closed; 8=year closed, 9=State, 10=average daily throughput of MSW (tons/day), 11=annual throughput of MSW (tons/year), 12=type of energy output, 13=gross $\mathrm{MW}_{\mathrm{e}}$ capacity, 14-net $M W_{e}$ capacity, 15=gross $\mathrm{kWh}_{\mathrm{e}} /$ ton of MSW, $16=$ net $\mathrm{kWh} /$ /ton of $\mathrm{MSW}$, $17=\mathrm{Btu} /$ ton of MSW, $18=$ census region, 19=NERC region, $20=$ sector(s) to which energy from MSW sold, and $21=$ sector code.

\footnotetext{
staff. .

${ }^{7}$ Determining what sector to allocate the energy production was based on the judgment of ORNL Energy Division
} 
Table 2E-1. Plant-Level Data from the GAA Database with Sector Codes Added

\begin{tabular}{|c|c|c|c|c|c|c|c|c|c|c|c|c|c|c|c|c|c|c|c|c|c|}
\hline 1 & 2 & 3 & 4 & 5 & 6 & 7 & 8 & 9 & 10 & 11 & 12 & 13 & 14 & 15 & 16 & 17 & 18 & 19 & 20 & & \\
\hline 1 & $8^{\circ}$ & 4 & 84 & · & & 3 & 86 & AI & 50 & 13000 & 1 & 0 & 0 & 0 & $0:$ & $\because$ & $\cdot 6$ & 9 & $i$ & & \\
\hline 2 & 5 & 7 & 90 & & . & & & AI & 586 & 221000 & 1 & 0 & 0 . & 0 & 0 & 0 & 6 & $?$ & $i$ & & \\
\hline 3 & 5 & 3 & 84 & & & & & AL & 290 & 100000 & 1 & 0 & 0 & 0 & 0 & 0 & 6 & 9 & $i$ & & \\
\hline$\cdot 4$ & $\dot{5}$ & 6 & 81. & . & & & & $\mathrm{AR}$ & 45 & 15435 & 5 & 0 & $0^{\circ}$. & .0 & 0. & 0 & 9 & 11 & $c^{\prime}$ & · & \\
\hline 5 & 4 & & & & 90 & & & $A K$ & 200 & 60000 & 4 & 0 & 0 & 0 & 0 & 0 & 9 & 11 & c & & \\
\hline 6 & 5 & 5 & 85 & & & & & $\mathrm{AK}$ & 20 & 5500 & 1 & 0 & 0 & 0 & 0 & $0^{-}$ & 9 & 11 & $c$ & & \\
\hline 7 & 5 & 5 & 81 & & & . & & $\mathrm{AR}$ & 94 & 22598 & 1 & 0 & 0 & 0 & 0 & 0 & 7 & .10 & $i$ & & \\
\hline 8 & 5 & 1 & 80 & $\cdot$ & & & & $\mathbf{A R}$ & 45 & 16200 & 1 & 0 & 0 & 0 & 0 & 0 & 7 & 10 & $i$ & & \\
\hline 9 & 5 & 5 & 87 & & & & & $\mathrm{CA}$ & 380 & $110000^{\circ}$ & 2 & 11.5 & 10 & 725 & 630 & 5600 & 9 & 13 & u & & \\
\hline 10 & 5 & 1 & 89 & & & & & $\mathrm{CA}$ & 730 & 293000 & 2 & 22.5 & 17 . &. & 450 & 4750 & 9 & 13 & u & & \\
\hline 11 & 4 & 12 & 88 & & & & & $\mathrm{CA}$ & 1170 & 427000 & 2 & 36 & 30 & & 540 & 4800 & 9 & 13 & $u$ & & \\
\hline 12 & 2 & - & & 10 & 94 & & & $C A:$ & 1822 & 638000 & 2 . & 41 & 36 & & 540 & $4900^{\circ}$ & 9 & 13 & u & & \\
\hline 13 & 8 & 11 & 84 & 7 & 91 & 5 & 85 & $\mathrm{CA}$. & .100 & 36500 & 3 & $1.54^{\prime}$ & 1.2 & & & 6500 & 9 & -13 & ue & & \\
\hline 14 & .5 & $7^{\circ}$ & 88 & & & & & $C T$ & 2000 & 720000 & 2, & 67 & $\cdot 60$ & 720 & 640 & 5300 & 1 & 7 & u & & \\
\hline 15 & 5 & 3 & 88 & & - & & & $C T$ & 600 & 195000 & 2 & 16 & 13.5 & 620 & 535 & 5000 & 1 & 7 & $\mathbf{u}$ & & \\
\hline 16 & .2 & & & 3 & 94 & & & $C T$ & 468 & 170638 & 2 & 14.5 & 12 & & 560 & 5300 & 1 & 7 & $\mathbf{u}$ & & \\
\hline 17 & 5 & 10 & $\cdot 88$ & & & & . & $C T$ & 2300 & 624000 & 2 & 90. & 68.5 & & & 5500 & 1 & .7 & $\mathbf{u}$ & & ? \\
\hline 18 & 2 & & & 3 & 93 & & & $C T$ & 425 & -131750 & 2 & 15 & 13 & 600 & 550. & 4500 & 1 & 7 & $\mathbf{u}$ & & . \\
\hline 19 & 3 & & & 10 & 91 & & & CT. & 522 & 190530. & .2 & 18 & 16 & & 520 & 5000 & 1 & 7. & $\mathbf{u}$. & & - \\
\hline 20 & 5 & 5 & 89 & & & 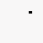 & & $C T$ & 380 & 138000 & 3 & 11 & 9.3 & $500^{\circ}$ & 384 & 4850 & 1 & ?: & $u i$ & & \\
\hline 21 & 5 & 11 & 81 & & & & & CT & 130 & 37000 & 3 & 2.2 & 1.9 & 150 & & 5000 & $i$ & 7 & $\mathbf{u}$ & & . \\
\hline 22 & 5 & 3 & 84 & & & & & $D E$ & 500 & 230000 & 4 & 0 & 0 & 0 & 0 &.${ }^{0}$ & 5 & 3 & $\mathbf{u}$ & & \\
\hline 23 & .5 & 11 & 87 & & & & & $\mathrm{DE}$ & 546 & 200000 & 3 & 13.3 & 20.5 & . & 532 & 5500 & 5 & 3 & $u i$ & & \\
\hline 24 & 5 & 10 & 87 & & & & & FL & $1200^{\circ}$ & 430000 & 2. & 30 & 27.5 & & 492 & 4500 & 5 & 8 & $\mathbf{u}$ & & . \\
\hline 25 & 3 & & & 1 & 92 & & & EI & 2250 & $\cdot 821250$ & 2 & 63.4 & 57 & 676 & 608 & 5200 & 5 & 8 & $\mathbf{u}$ & & \\
\hline 26 & 8 & 4 & 89 & $\because$ & & 9 & 89 & FL & 540 & 140400 & 4 & 0 & 0 & 0 & 0 & 0 & 5 & 8 & $i$ & & $\cdot$ \\
\hline 27 & 5 & 12 & 86 & & & & $\cdot$ & FL. & 130 & 46538 & 2 & 2.6 & 2.2 & & 300 & 5000 & 5 & 8 & us & & \\
\hline 28 & .5 & 7 & 83 & & & & & FE" & 275 & 63250 & 2 & 364 & 335 & - & & $4500^{\circ}$ & 5 & 8 & $\mathbf{u}$ & & \\
\hline 29 & 2 & & . & 1 & 94 & & & FI & 1530. & $558450^{\circ}$ & 2 & 50 & 47 & & 630 & 5000 & 5. & 8 & $\cdot \mathbf{u}$ & & \\
\hline 30 & 5 & 12 & 79 & & & & & EL & 33 & $: 10296$. & 1 & 0 & 0 & .0 & 0 & 0 & 5 & 8 & $i$ & - & \\
\hline 31 & 5 & $\cdot 1$ & 82 & $\cdot$ & & & & EL & 2800 & 1022000 & 2 & 77 & 62 & 480 & 388 & 5000 & 5 & 8 & $\mathbf{u}$ & $\cdot$ & \\
\hline 32 & 6 & 11 & 83 & & & & & FL & 25 & 9125 & 1 & 0 & 0 & 0 & 0 & 0 & 5 & 8 & c & & \\
\hline 33 & 4 & & & & 91 & & & FL & 449 & 163000 & 2 & 14.5 & 10 & 25 & & 5000 & 5 & 8 & $\mathbf{u}$ & & \\
\hline 34 & 5 & .6 & 87 & & & & & FL & 435 & 186642 & 2 & 12 & 10. & $480^{\circ}$ & 432 & 4600 & 5 & 8 & $u$ & & \\
\hline 35 & $: 3$ & & & & 92 & & & FI & 660 & 207000 & 4 & 0 & 0 & 0 & 0 & 0. & 5 . & 8 & $i$ & & \\
\hline 36 & 5 & 5 & 83 & & & & & FL & 2835 & $920000^{\circ}$ & 2 & 62 & 55.8 & & 430 & 4000 & .5 & 8 & $\mathbf{u}$ & & \\
\hline 37 & 3 & & & 1 & 92 & . & · & FL & 2250 & 821250 . & 2 & $66 . \dot{5}$ & 60 & 709 & 638 & 5200 & 5 & 8 & $u$ & & \\
\hline 38 & 5 & 11 & 89 & & & & & FL" & 2000 & 624000 & 2 . & 61 & 49 & 600 & 500. & -4865 & 5 & 8 & us & 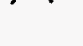 & \\
\hline 39 & 3 & & & & 91 & & & FL & 893 & 325945 & 2 & 31 & 29 & 650 & 550 & 4800 & 5 & 8 & $\mathbf{u}$ & & \\
\hline 40 & 5 & .9 & 85 & & & & : & EL & 844 & 306000 & 2 & 17 & 15 & & 450 & 5000 & 5 & 8 & $\mathbf{u}$ & & \\
\hline 41 & 5 & 6 & 87 & & & & & GA & 480 & 175200 & -1 & 0 & 0 & 0 & .0 & 0 & 5 & 9 & $i$ & & 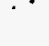 \\
\hline 42 & 5 & 5 & 90 & & . & & & HI & 1740 & 600000 & 2. & 55 & .46 & 550 & & 4800 & 9 & 13 & $\mathbf{u}$ & & \\
\hline
\end{tabular}


Table 2E-1. Plant-Level Data from the GAA Database with Sector Codes Added (Continued)

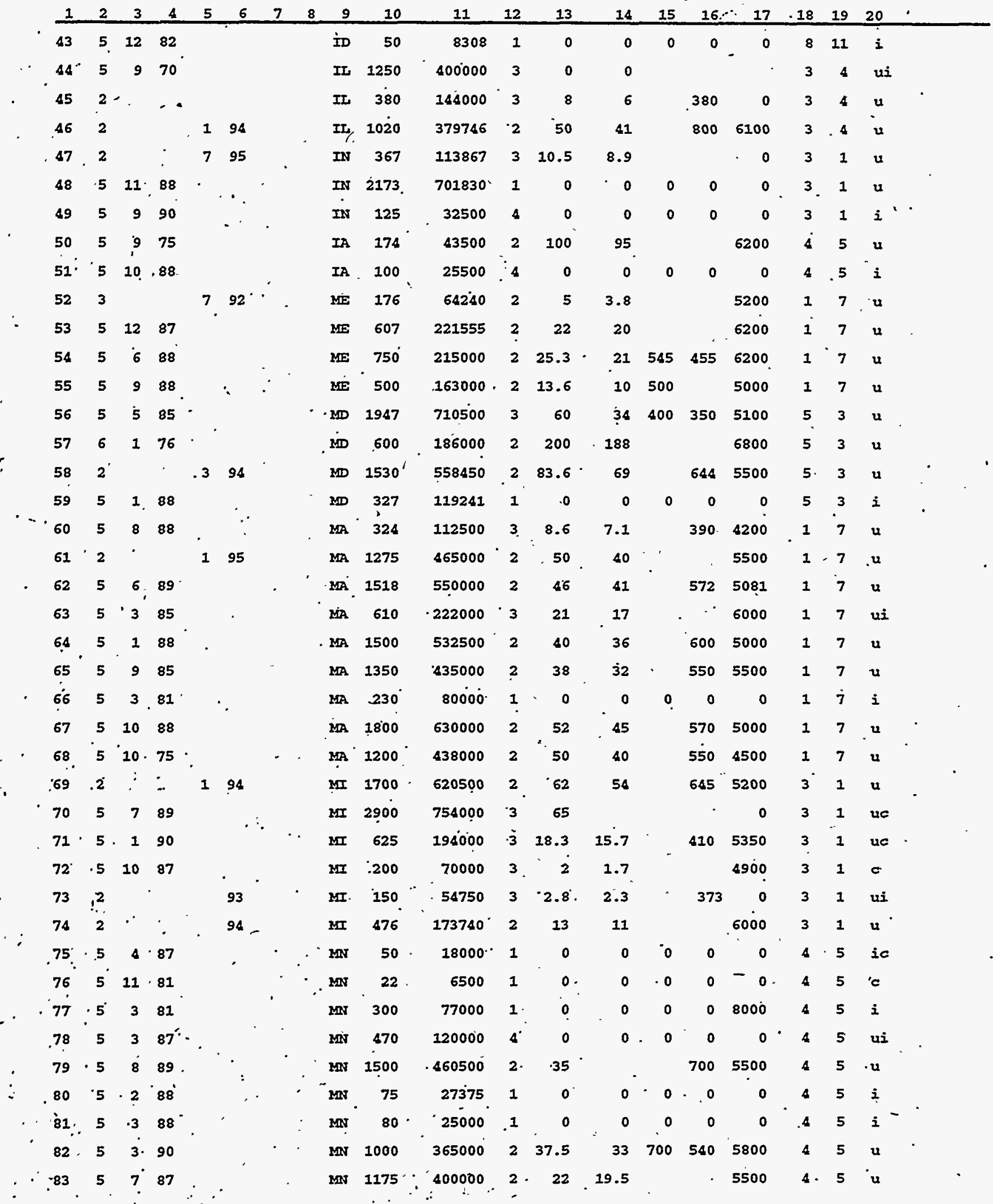


Table 2E-1. Plant-Level Data from the GAA Database with Sector Codes Added (Continued)

\begin{tabular}{|c|c|c|c|c|c|c|c|c|c|c|c|c|c|c|c|c|c|c|c|c|c|}
\hline $1^{\prime}$ & 2 & 3 & 4 & 5 & 6 & 7 & 8 & 9 & 10 & 11 & 12 & 13 & 14 & $15^{\circ}$ & $16 \ldots$ & $\therefore \quad 17$ & .18 & 19 & 20 & & \\
\hline 84 & -5 & 9 & 86 & & . & & & MEN & 100 & 33000 & 1 & 0 & 0 & 0 & 0 & 0 & 4 & 5 & $i$ & & \\
\hline 85 & 5 & 9 & 82 & & & & & MN & 85 & 22000 & 1 & 0 & 0 & 0 & 0 & 0 & 4 & 5 & $i$ & . & \\
\hline 86 & $5^{\circ}$ & 3 & 87. & &. & & & MN & 190 & 59000 & 3 & 3. & 2.25 & 293 & & $5500 \backslash$ & 4 & 5 & $u^{\prime}$ & & \\
\hline 87 & 2 & & & 3 & 93 & & & MN & 640 & 233600 & 2 & 23 & 20 & & 550 & 5000 & 4 & 5 & $u$ & & \\
\hline 88 & 5 & 12 & 82 & & & & & MN & 68 & 22440 & 1 & 0 & 0 & 0 & 0 & 0 & 4 & 5 & i & & \\
\hline 89 & 5 & 11 & 85 & & & & & MN & .50 & 10000 & 4 & 0 & 0 & 0 & 0 & 0 & 4 & 5 & c & & \\
\hline 90 & 5 & 1 & 85 & & & . & & MS & 120 & 37000 & 1 & 0 & 0 & 0 & $0^{\circ}$ & 0 & 6 & 10 & $i$ & $\cdots$ & \\
\hline 91 & 5 & 3 & 82 & & & & & MO & 30 & 10950 & .1 & 0 . & 0 & 0 & 0 & 0 & $4:$ & 10 & $\mathbf{i}$ & & \\
\hline 92 & 6 & 5 & 82 & & & - & & $\mathbf{M T}$ & 75 & 15000 & 1 & 0 & 0 & 0 & 0 & 0 & 8 & 11 & $\mathbf{u}$ & . & \\
\hline 93 & 2 & & & 1 & 95 & - & & NV & 712 & 260000 & 3 . & $50 े$ & 40 & & - & 0 & 8. & 11 & $\mathbf{u}$ & & \\
\hline 94 & $5^{-}$ & 3 & 87 & & * & & & NHA. & 180 & 65000 & 2 & 4.5 & 3.8 & 440 & & 5400 & 1 & 7 & u & & . \\
\hline 95 & 5 & $\dot{9}$ & .80 & & & & & $\mathrm{NH}$ & 100 & 36500 & 1 & 0 & 0 & 0 & 0 & 0 & 1 & 7 & c & • & \\
\hline 96 & 2 & & & & 94 & & & $\mathrm{NHH}$ & 504 & 183960 & 2 & 14 & 12.5 & & .425 & 4500 & 1 & 7 & u & & \\
\hline 97 & 5 & 9 & 89 & & & & & NH & .425 & 155000 & 2 & 13 & 12 & 550 & 470 & 5000 & .1 & 7 & u & & \\
\hline 98 & 3 & & & 3 & 91 & & & NS & 840 & 306600 & 2 & 30 & 21 & & 482 & 4500 & 2 & 3 & $\mathbf{u}$ & & \\
\hline 99 & 2 & & . & 10 & 93 & & & No & -830. & 302500 & 2 & 36 & 32 & 655 & 560 & 5000 & 2 & 3 & $\mathbf{u}$ & $\cdot$ & \\
\hline 100 & 2 & & & & 93 & & & NW & 1265 & 438000 & 2 & 45 & 38.25 & & 455 & 4500 & 2 & 3 & $\mathbf{u}$ & & : \\
\hline 101 & 3 & & & -10 & 90. & & & NJ & 2000 & 725000 & 2 & 76 & 72 & 501 & - & 4500 & .2 & 3 & $\mathbf{u}$ & & \\
\hline 102 & 5 & 7 & 88 & & - & & & NNT & 450 & 124100 & 2 & $13.5^{\circ}$ & 10.5 & & 482 & 4650 & 2 & 3 & $\mathbf{u}$ & & \\
\hline 103 & 2 . & & & 9 & $93^{\circ}$ & & & NW & 1219 & 400000 & 2 & 45 & 37.3 & 753 & 625 & 5500 & 2 & 3 & $u$ & & \\
\hline 104 & 3 & & & & 94 & & & NJ & 425 & 156000 & 2 & 12.5 & 9.8 & & 425 & 5200 & 2 & 3 & u & & \\
\hline 105 & 2 & & & 1 & 93 & & & NW & 1200 & 437000 & 2 & 44 & 39 & 670 & 567 & 5400 & 2 . & 3 & $\mathrm{u}$ & & \\
\hline 106 & .2 & & & & 95 & & & No & 2400 & 876000 & 2 & 88. & 80 & & 482 & 4500 & 2 & 3 & $\mathbf{u}$ & & \\
\hline 107 & 2 & & & 10 & 94 & . & & NT & 1166 & 425517 & 2 & 40 & 34 & $53 \dot{5}$ & & 5500 & 2 & 3 & $\dot{u}$ & & \\
\hline 108 & 2 & & & 7 & .95 & & & $N T$ & 1445 & 527425 & 2 & 63 & 57 & & & 4950 & 2 & 3 & u & · & \\
\hline 109 & 5 & 5 & $90^{\circ}$ & & $=$ & & & $T N T$ & 525 & 165000 & 2 & 14. & 12 & 475 & 425 & 4500 & 2. & 3 & u & & \\
\hline 110 & 5 & 12 & 86 & & - & . & & NJ & 60 & $1560^{\circ}$ & 1 & 0 & 0 & 0. & $\dot{0}$ & 0 & 2 & 3 & $i$ & & \\
\hline 111 & 5 & 2 . & 81 & & & & & NYY & 720 & 148000 & 4 & 0 & 0. & 0 . & 0 & 0 & 2 & 6 & e & & \\
\hline 112 & 5 & 4 & 81 & & & & & $\mathbb{N Y Y}$ & 500 & 147000 & 1 & 0 & 0 & 0 & 0 & -0 & 2 & 6 & c & & \\
\hline 113 & 5 & 4 & 89 & & & & & NY & 640 & 210000 & 2 & 17 & 14 & & $\$ 10$ & 5000 & 2 & 6 & $\cdot \mathbf{u}$ & & \\
\hline 114 & 2 & & . & 1 & -94 & & & NY & 1275 & 465000 & 2 . & 50 & 40 & & $\cdot$ & 5500 & 2 & 6 & $u^{\circ}$ & & . \\
\hline 115 & 2. & & - & & 95 & & & $\mathbf{W Y} \mathbf{Y}^{\circ}$ & 2550 & 930750 & 1 & 0 & 0 & 0 & 0 & 0 & 2 & 6 & c & & \\
\hline 116 & 5 & 2 & 83 & & & . & & $\mathrm{NY}$ & 130 & 46500 & 1 & 0 & 0 & 0 & 0 & .0 & 2 & 6 & $i$ & & \\
\hline $117^{\circ}$ & 3 & · & & 3 & 92 & & & $\mathbb{N Y}$ & 638 & 253000 & 2 & 25 & 21 & 736 & 627 & 6000 & 2 & $6^{\circ}$ & $\mathbf{u}$ & & \\
\hline 118 & 5 & $\checkmark 8$ & 83 & & & & & NY & 225 & $78750^{\circ}$ & 3 & 2.5 & 1 & & & 5000 & 2 & 6 & ui & & • \\
\hline 119 & 3 & & & 10 & .91 & & & NY. . & $345=$ & 126000 & 2 & 13 & 11 & - & & 5500 & $2^{\prime}$ & 6 & u & - & \\
\hline 120 & 5 & 6 & 89 & & & . & & พับ & 450 & 1600.00 & 2 & $11: 5$ & 8 & & 370 & 4450 & 2. & 6 & $\mathbf{u}$ & & \\
\hline 121 & 2 & . & & 2. & .93 & & & $\mathbf{N I}$. & 485 & 165000 & 2 & $18^{\circ}$ & 15 & 560 & 467 & 5200 & 2 & 6 & $\mathbf{u}$ & & \\
\hline 122 & 5 & 2 . & 88 & & & & & $\mathbb{N X}$ & 170 & 62050 & 2 & 4.5 & 3 & & $\therefore$ & 5000 & 2 & 6 & $\mathbf{u}$ & & \\
\hline 123. & 5 & 12 & 80. & - & $\cdot$ & & & $\mathbf{N Y}$ & 1800 & 583900 & 3 & 50 & 30 & & & ? & 2 & 6 & ui & & \\
\hline 124 & 2 & & & 10 & .93 & & & $\mathbf{N Y}$ & 850 & 300000 & 2 & 31 & 27 & & & 6000 & 2 & 6. & $\mathbf{u}$ & . & \\
\hline
\end{tabular}


Table 2E-1. Plant-Level Data from the GAA' Database with Sector Codes Added (Continued)

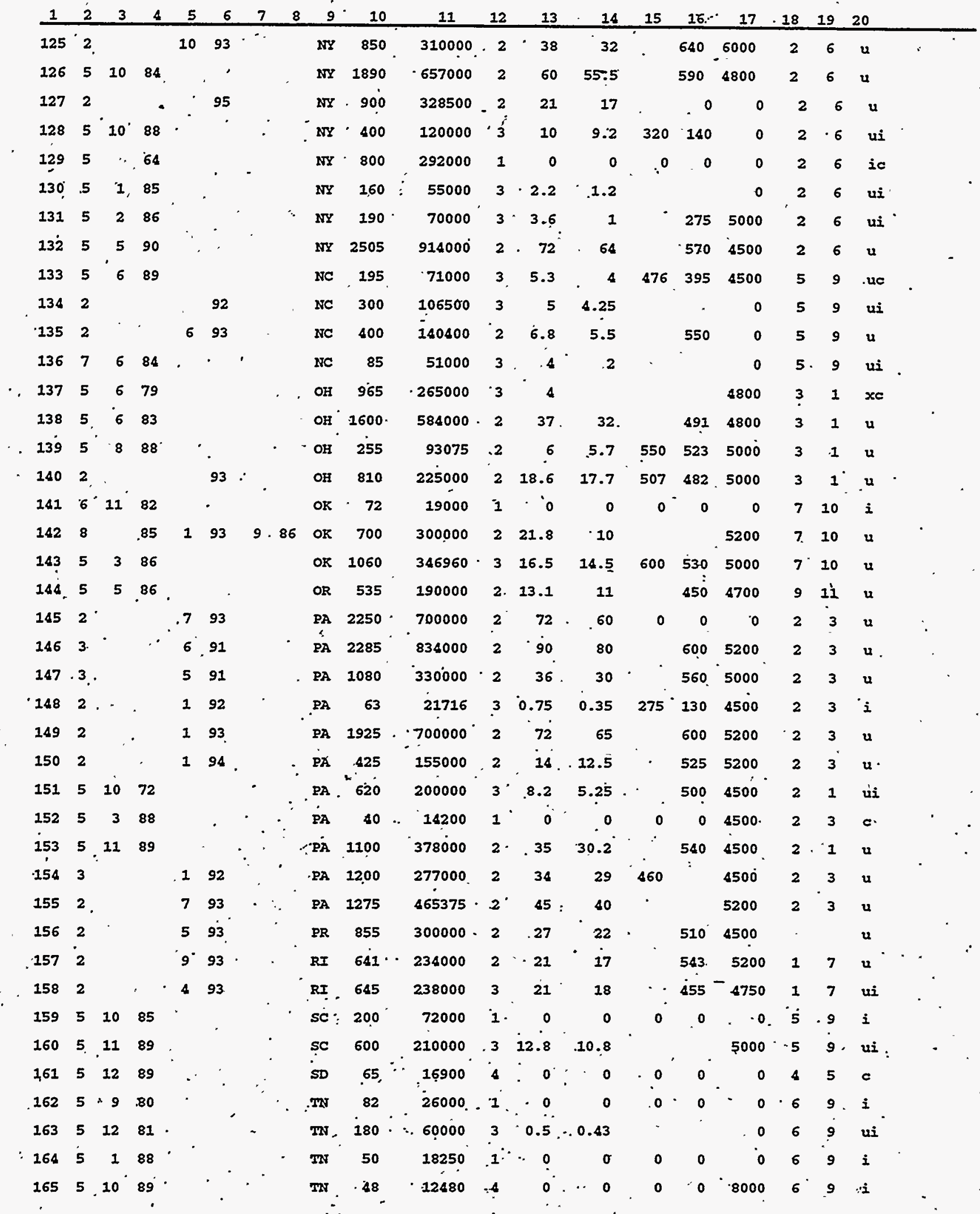


Table 2E-1. Plant-Level Data from the GAA Database with Sector Codes Added (Continued)

\begin{tabular}{|c|c|c|c|c|c|c|c|c|c|c|c|c|c|c|c|c|c|c|c|c|c|c|}
\hline 1 & 2 & 3 & 4 & 5 & 6 & 7 & 8 & 9 & 10 & 11 & 12 & 13 & 14 & 15 & 16. & 17 & .18 & 19 & $20^{\circ}$ & & & \\
\hline 166 & 3 & & & 12 & 92 & & & $T N$ & 205 & 7500.0 & 3 & 4 & 3.25 & & & 6000 & 6 & 9 & ui & & & \\
\hline 167 & 5 & 2 & 74 & & & . & & $\mathrm{TN}$ & 950 & 346750 & 3 & .7 .3 & 2.9 & & & 4900 & 6 & 9 & uc & & & \\
\hline 168 & 4 & 10 & 90. & & & & & $T N$ & 50 & 13000 & 4 & .0 & 0 & 0 & 0 & 0 & 6 & 9 & $i$ & & & \\
\hline 169 & 2 & & & 7 & 92 & 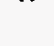 & & TN & 170 & 62050 & 1 & 0 & 0 & 0 & 0 & 0 & 6 & 9 & $i$ & & & \\
\hline 170 & 5 & 1 & 86 & & & & & $\mathbf{T X}$ & 39 & 13767 & 1 & 0 & 0 & 0 & 0 & 0 & 7 & 2 & $i$ & & & \\
\hline 171 & 5 & 8. & 86 & & & & & $\mathbf{T X}$ & 35 & 8190 & 1 & 0 & 0 & 0 & 0 & 0 & 7 & 2 & $i$ & & & \\
\hline 172 & 5 & 3 & 86 & & & & & $\mathrm{TFX}$. & 85 & 20400 & 2 & 0.86 & 0.66 & $\cdot$ & & 4500 & 7 & 2 & u & $\cdot$ & & \\
\hline 173 & 5 & 2 & 80 & & & $\cdot$ & & $T X$ & 4 & 832 & 1 & .0 & 0 & 0 & 0 & 0 & 7 & 2 & c & & & \\
\hline 174 & 5 & 2 & 80 & & & & & $\mathrm{TX}$ & 9 & 1872 & 1 & 0 & 0 & 0 & 0 & 0 & 7 & 2 & e & & & \\
\hline 175 & 6 & 6 & 82 & & . & & . & $T X$ & 48 & 14736 & 1 & 0 & 0 & 0 & 0 & 0 & 7 & 2 & $i$ & & & \\
\hline 176 & 5 & 10 & 88 & & & & & UT & 350 & 127750 & 1 & 0 & 0 & 0 & 0 . & 0 & 8 & 11 & $i$ & & & \\
\hline 177 & 5 & 2 & 88 & . & & & & $\mathrm{VA}$ & 940 & -338400 & 2 & 22 & $19.8^{\circ}$ & 520 & 470 & 4800 & 5 & 1 & $u$ & & & \\
\hline 178 & 5 & 1 & 86. & & & & - & $\mathrm{VA}$ & $\$ 2$ & 8400 & 1 & 0 & 0 & 0 & 0 & 0 & 5 & 9. & $i$ & & & \\
\hline 179 & 5 & 10 & 80 & & & & & $\mathrm{VA}$ & 200 & 72500 & 1 & 0 & 0 & 0 & 0 & 0 & 5 & 9 & c & & & \\
\hline 180 & 5 . & 11 & 82 & & & & & VA & 65 & 23725 & 1 & 0 & 0 & 0 & 0 & 0 & 5 & 9 & c & & & \\
\hline 181 & 5 & 6 & 90 & · & & - & & $\mathrm{VA}^{\circ}$ & $2700^{\circ}$ & 985150 & 2 & 85. & 73 & 610 & 540 & 4400 & 5 & 1. & $\mathbf{u}$ & . & & \\
\hline 182 & 8 & 5 & 67 & & 94 & 10 & 86 & VA & 150 & 54750 & 1 & 0 & 0 & 0 & 0 & 0 & 5 & 9 & $i$ & & & \\
\hline 183 & 2 & . & & & 93 & & & $\mathrm{VA}$ & 700 & 182000 & 2 & 12 & 9 & . & . & 0 & 5 & 9 & u & & & \\
\hline 184 & 5 & 1 & 88 & & & & & VA & 1400 & 364000 & 3 & 40 & 35 & & 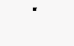 & 5550 & 5 & 9 & i & & & \\
\hline 185 & 5 & 7 & 78 & & & & & $\nabla A$ & 90 & 23400 & 1 & 0 & 0 & 0. & 0 & 0 & 5 & 1 & - $i$ & & & \\
\hline 186 & 8 & 1 & 88 & 7 & 91. & 8 & 88 & $\mathbf{V T}$ & 204 & $75000^{\circ}$ & 2 & 7 & 6 & & 470 & 0 & 1 & 5 & $\mathbf{u}$ & • & & \\
\hline 187 & 5 & 11 & 86 & & . & & & WA & 100 & 35000 & 2 & 1.5 & 1 & & 350 & 4500 & 9 & 11 & $\mathbf{u}$ & & & \\
\hline 188 & 3 & & & 1 & 92 & . & & WA. & 110 & 40150 & 1 & 0 & 0 & 0 & 0 & 0 & 9 & 11 & $i$. & . & & \\
\hline 189 & 2 & & & & 95. & & & WA & 2200 & $\cdot 803000$ & 2 & 90 & 77 & · & 565 & 5000 & 9 & 11 & $\mathbf{u}$ & & . & \\
\hline 190 & 5 & 7 & 88 & . & & & & WA & 150 & .54000 & 2 & 2.4 & 2.04 & & 345 & 4500. & 9. & 11 & $\mathbf{u}$ & & & \\
\hline 191 & 3. & & & 8 & 91 & . & & WA & 680 & 248200 & 2 & 26 & .22 .1 & & 497 & 0 & 9 & 11 & $\mathbf{u}$ & & & \\
\hline 192 & 5 & 7 & 79 & & & . & $\cdot$ & WA & 450 & 117000 & 4. & $\dot{0}$ & 0 & 0 & 0 & 0 & 9 & 11 & $\mathbf{u}$ & & & \\
\hline 193 & 5 & 3 & 90 & & & . & & $W A$ & 250 & 100000 & 2 & 50 & 43 & & & 5600 & 9 & .11 & $\mathbf{u}$ & & & \\
\hline 194 & 3 & & & & -91 & & & WV & 550 & 132000 & 4 & 0 & 0 : & 0 & 0 & 0 & .5 & 1 & $i$ & $\cdot$ & & \\
\hline 195 & 5 & 10 & 86 & & & & & WI & 80 & 29000 & 3 & 0.27 & 0.07 & & & 4750 & .3 & 5 & ui & · & & \\
\hline 196 & 5 & $7^{\prime}$ & 88 & & & . & & WI & 225 & 56000 & 2 & 30 & 28 & & & 5500. & 3 & 4 & $\mathbf{u}$ & & 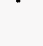 & \\
\hline 197 & 5 & 1 & 79 & & & $\therefore$ & & WI & 250 & 65000 & 2 & 100 & & & & 5759 & 3 & 4 & $\mathbf{u}$ & . & . & . \\
\hline 198 & 5 & .5 & $89^{\circ}$ & & & & & WI & 80 & 22880 & 3 & 1.4 & 0.8 & 150 & 100 & 5450 & 3 & 4 & ui & & & \\
\hline 199 & 5 & 3 & 89. & & & & & $W I$ & 105 & 33600 & 3 & 1.2 & 0.7 & 110. & 85 & $\overline{5000}$ & 3 & 5 & uc & . & & \\
\hline 200 & 2 & & & & 93 & & & WI & 128 & 46500 & 2 & 2.75 & 2.5 & 323 & .263 & 5000 & 3 & 4 & u & & · & \\
\hline 201 & $5^{\circ}$ & 6 & 79 & & & & & WI & 143 & 51480 & 1 & 0 & 0. & 0 & 0 & 0 & 3 & 4 & $i$ & & & \\
\hline 202 & 2 & & & 1 & 94 & ' & & WI & 510 & 219000 & 3. & & & . & & 5500 & 3. & 4. & $\mathbf{u}$ & & & \\
\hline
\end{tabular}


Table 2E-2. Plant-Level Data with Missing Values Filled In and Sector Code Added

\begin{tabular}{|c|c|c|c|c|c|c|c|c|c|c|c|c|c|c|c|c|c|c|c|c|c|c|c|c|}
\hline 1 & $2^{-}$ & . 3 & 4 & 5 & 6 & 7 & 8 & 9 & 10 & 11. & 12 & \multicolumn{2}{|r|}{13} & \multicolumn{2}{|r|}{14} & 15 & 16 & 27 & 18 & 19 & 20 & 21 & & \\
\hline 66 & 5 & 3 & 81 & & & & & MA & 230 & 80000 & 1 & & 0 & & 0 & 0 & $0 .-\cdots$ & 5253 & 1 & 7 & $i$ & 2 & & \\
\hline 95 & $5:$ & 9 & 80 & & & & & NH & 100 & 36500 & 1 & & 0 & & 0 . & 0 & 0 & 5253 & 1 & 7. & $c$ & 1 & & \\
\hline 112 . & 5 & 4 & 81 & & & & & $\mathrm{NY}$ & 500. & 147000 & 1 & & 0 & & 0 & 0 & 0 & 5253 & 2 & 6 & $c$ & 1 & & \\
\hline 110 & $\cdot 5$ & 12 & 86. & & & & & NJ & 60 & 15600 & 1 & & 0 & & 0 & 0 & 0 & 5253 & 2 & 3 & $i$ & 2 & & \\
\hline 115 & 2 & & & .1 & 95 & & $\cdot$ & iv & 2550 & 930750 & 1 & & 0 & & 0 & 0 & 0 & 5253 & 2 & 6 & c & 1 & & \\
\hline 152 & 5 & 3 & 88 & & & & & $\mathbf{P A}$ & 40 & 14200 & 1 & & 0 & & 0 & 0 & 0 & 4500 & 2 & 3 & c & $1^{*}$ & & \\
\hline 116 & 5 & 2 & 83 & - & & & & NY & 130. & 46500 & 1 & & 0 & & 0 & 0 & 0 & 5253 & 2 & 6 & $i$ & 2 & . & " \\
\hline 129 & 5 & & 64 & & & $\cdot$ & & WY & 800 & 292000 & 1 & & .0 & & 0 . & 0 & 0 & 5253 & 2 & 6 & ic & $\dot{4}$ & & \\
\hline 201 & 5 & 6 & 79 & & & & & WI & 143 & 51480 & 1 & & 0 & & 0 & 0 & 0 & 5253 & 3 & 4 & $i$ & 2 & - & \\
\hline 48 & 5 & 11 & 88 & & & & & IN & 2173 & 701830 & $i$ & . & 0 & & 0 & 0 & 0 & 5253 & 3. & 1 & น & 3 & & \\
\hline 76 & 5 & 11 & 81 & & & & & $\ln$ & 22 & 6500 & 1 & & 0 & . & 0 & 0 & 0 & 5253 & 4 & 5 & $c$ & $\cdot 1$ & & \\
\hline 81 & 5 & $3^{x}$ & 88 & . & & & & InN & 80 & -25000 & 1 & & 0 & & 0 & 0 & $=0$ & 5253 & 4. & 5 & $i^{\circ}$ & 2 & & \\
\hline 84 & 5 & 9 & 86 & & & $\bullet^{\circ}$ & & InN & 100 & 33000 & 1 & & 0 & & 0 & 0 & 0 & 5253 & 4 & 5 & $i$ & 2 & & \\
\hline 85 & 5 & $\dot{9}$ & 82 & & & & & MNN & 85 & 22000 & 1 & & 0 & • & 0 & 0 & 0 & 5253 & 4 & 5 & $i$ & 2 & & \\
\hline 88 & 5 & 12 & 82 & & " & & & $r^{\text {MNN }}$ & 68. & 22440 & 1 & & 0 & & 0 & 0 & 0 & 5253 & 4 & 5 & $i$ & 2 & & = \\
\hline 80 & 5 & 2. & 88 & & & & & $\mathbf{M N}$ & 75 & 27375 & 1 & & 0 & & 0 & 0 & 0 & 5253 & 4 & 5 & $i$ & 2 & $\therefore$ & \\
\hline 91 & 5 & 3 & 82 & $\cdot$ & . & & & MO & 30. & 10950 & 1 & & 0 & & 0 & 0 & 0 & 5253 & 4 & 10 & $\mathbf{i}$ & 2 & & \\
\hline 77 & 5. & 3 & 81 & & & & & inis & 300 & 77000 & 1 & & 0 & & 0 & 0 & 0 & 8000 & 4 & 5 & $i$ & 2 & & \\
\hline 59. & 5 & 1 & 88 & & $r$ & & & MD & 327 & 119241 & 1 & & 0 & & 0 & 0 & 0 & 5253 & 5 & 3 & $i$ & 2 & & \\
\hline 185 & 5 & 7 & 78 & & & - & & VA & 90. & 23,400 & 1 & & 0 & ' & 0 & 0 & 0 & 5253 & 5 & 1 & $i$ & 2 & & \\
\hline 159 & 5 & 10 & 85 & & & & . & sc & 200 & 72000 & 1 & & 0 & . & 0 & 0 & 0 & 5253 & 5 & 9 & $i$ & 2 & & \\
\hline 32 & $6^{\prime \prime}$ & 11 & 83 & & & . & 。 & EL & 25 & 9125 & 1 & & 0 & & 0 & 0 & 0 & 5253 & 5 & -8 & c. & $\cdot 1$ & $=$ & \\
\hline 178 & 5 & 1 & 86 & $x^{2}$ & & & & $\begin{aligned} & \text { VA } \\
= & \end{aligned}$ & 42 & 8400 & 1 & & ? & & 0 & 0 & 0 & 5253 & 5 & 9 & i & 2 & & \\
\hline 179 & 5 & 10 & 80 & & . & & 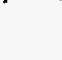 & VA & 200 & 72500 & 1 & & 0 & & 0 & 0 & 0 & 5253 & 5 & 9 & $c$ & 1 & & \\
\hline 41 & 5 & 6 & 87 & • & & * & & GA & 480 & 175200 & 1 & & 0 & & 0 & 0 & 0 & 5253 & 5 & 9 & $i$ & 2 & .* & \\
\hline 182 & 8 & 5 & 67 & & 94 & 10 & 86 & $\mathbf{V A}$ & 150 & 54750 & 1 & " & $\dot{0}$ & & 0 & 0 & 0 & 5253 & 5 & 9 & $i$ & 2 & & \\
\hline 180 & 5 & 11 & 82 & . & & & & VA & .65 & 23725 & 1. & & 0 & & 0 & 0 & 0 & 5253 & 5 & 9 & $c$ & 1 & & \\
\hline 90 & 5 & 1 & -85 & $=$ & $\cdot$ & & $\cdot$ & MS & 120 & 37000 & 1 & & 0 & & 0 & 0 & 0 & 5253 & 6 & 10 & $i$ & 2 & * & \\
\hline 1 & 8 & 4 & 84 & & $\dot{.}$ & 3 & 86 & ar & 50 & 13000 & 1 & & .0 & & 0 & 0 & 0 & 5253 & 6 & 9 & $i$ & 2 & & \\
\hline 3 & 5 & 3 & 84 & & & & & AंI & 290 & 100000 & $\times 1$ & & 0 & & 0 & 0 & 0 & 5253 & 6 & 2 & $i$ & 2 & . & \\
\hline 2 & 5 & 7 & 90 & & & & $\cdot \cdot$ & AT & 586 & 221000 & 1 & . & $0:$ & & 0 & .0 & 0 & 5253 & 6 & 9 & $i$ & 2 & & \\
\hline 164 & 5 & 1 & 88 & & - & & & MN & 50 & 18250 & 1 & & 0 & $\cdot$ & 0 & 0 & 0 & 5253 & 6 & 9 & $i$ & 2 & & \\
\hline 162 & 5 & 9 & 80 & . & $\cdot$ & & & $m$ & 82. & 26000 & 1 & & ó & & $0:$ & 0 & 0 & 5253 & 6 & 9 & $\dot{I}$ & 2 & & \\
\hline 169 & 2 & & & 7 & 92 & & & TN: & 170 & 62050 & 1 & . & 0 & & 0 & 0 & $0^{\circ}$ & 5253 & 6 & 9 & $i$ & 2 & & \\
\hline 175 & 6 & 6 & 82 & & & & & $\mathrm{TXX}$ & 48 & 14736 & 1 & & 0 & & .0 & 0 & 0 & 5253 & 7 & 2 & $i$ & 2 & ' & \\
\hline 7 & 5 & 5 & 81 & & & - & & $\mathrm{AR}$ & 94 & 22598 & 1 & ' & 0 & & 0 & 0. & 0 & 5253 & 7 & 10 & $i$ & 2 & & \\
\hline 141 & .6 & 11 & 82 & & & & & OK & .72 & 19000 & 1 & & 0 & & 0 & 0 & .0 & 5253 & 7 & 10 & i & 2 & & \\
\hline 8 & 5 & 1 & 80 & & & & & AR & 45 & 16200 & 1 & & 0 & & 0 & 0 & 0 & 5253 & 7 & 10 & $i$ & 2 & & \\
\hline
\end{tabular}


Table 2E-2. Plant-Level Data with Misșing Values Filled In and Sector Code Added (Continued)

\begin{tabular}{|c|c|c|c|c|c|c|c|c|c|c|c|c|c|c|c|c|c|c|c|c|c|}
\hline 1 & 2 & 3 & 4 & 5 & 6 & 7 & 8 & 9 & 10 & $11^{\circ}$ & 12. & 13 & 14. & 15 & 16. & $=17$ & 18. & 19 & 20 & 21 & \\
\hline 171 & 5 & 8 & 86 & & & & & $\mathbf{T X}$ & 35 & 8190 & 1 & 0 & 0 & 0 & 0 & 5253 & 7 & 2 & $i$ & 2 & \\
\hline 170 & 5 & 1 & 86 & & & & &.$T X$ & 39 & 13767 & 1 & 0 & 0 & 0 & 0 & 5253 & 7 & 2 & i. & 2 & \\
\hline 174 & 5 & $\dot{2}$ & 80. & & & & & $T X$ & 9 & 1872 & 1. & 0 & 0. & 0 & 0 & 5253 & 7 & 2 & $c$ & 1 & \\
\hline 173 & 5 & 2 & 80 & & & & & $\mathbf{T X}$ & 4 & 832 & 1 & 0 & 0 & 0 & 0 & 5253 & 7 & 2 & c & 1 & \\
\hline 92. & 6 & 5 . & 82 & & & & & MT & 75 & 15000 & 1 & 0 & 0 & 0 & 0 & 5253 & 8 & 11 & $\mathbf{u}$ & 3 & \\
\hline $43^{\circ}$ & 5. & 12 & 82 & & & & & ID & 50 & 8308 & 1 & 0 & . & 0 & 0 & 5253. & 8 & 11 & $i$ & 2 & \\
\hline 176 & 5 & 10 & 88 & $\cdot$ & $\cdot$ & & & UT & 350 & 127750 & 1 & 0 & 0 & 0 & 0 & 5253 & 8 & 11. & $i$ & 2 & \\
\hline 188. & 3 & & & $\dot{1}$ & 92 & & & WA & 110 & 40150 & 1 & 0 & ? & 0 & 0 & 5253 & 9 & 11 & $i$ & 2 & \\
\hline 6. & 5 & 5 & 85 & & & & & $\mathrm{AK}$ & 20 & 5500 & 1 & 0 & 0 & 0 & 0 & 5253 & .9 & 11 & $c$ & 1 & \\
\hline 156 & 2 & & & 5 & 93 & & & PR & 855. & 300000 & 2 & 27 & 22 & 626 & 510 & 4500 & & & $\mathbf{u}$ & 3 & \\
\hline 61. & 2 & & & 1 & 95 & & & MA & 1275 & 465000 & 2 & 50 & 40 & 620 : & 496 & 5500 & 1 & 7 & $\mathbf{u}$ & 3 & \\
\hline 68 & 5 & 10 & 75 & & & & & MA & 1200 & 438000 & 2 & 50 & 40 & 688 & 550 & 4500 & $i$ & 7 & $\mathbf{u}$ & 3 & \\
\hline 62 & 5 & 6 & 89 & & & & & $\mathbf{M A}$ & 1518 & 550000 & 2 & 46 & 41 & 642 & 572 & 5081 & 1 & 7 & $\mathbf{u}$. & 3 & \\
\hline 65 & 5 & 9 & 85 & & & & & MA & 1350 & 435000 & 2 & 38 & 32. & 653 & 550 & 5500 & 1 & 7 & u & 3 & \\
\hline 64 & 5 & 1 & 88 & & & & & $M A$ & 1500 & 532500 & 2 & 40 & 36 & 667 & 600 & 5000 & 1 & 7 & $\mathbf{u}$ & 3 & \\
\hline 67 & 5 & 10 & 88 & & & & . & MA & .1800 & 630000 & 2 & 52 & 45 & 659 & 570 & 5000 & 1 & 7 & $\mathbf{u}$ & 3 & \\
\hline 97 & 5 & 9 & 89 & & & & & $\mathbf{N H}$ & 425. & 155000 & 2 & 13 & .12 & 550 & .470 & 5000 & 1 & 7 & $u$ & 3 & \\
\hline 96 & 2 & & & & 94 & & & $\mathbf{N H}$ & 504 & 183960 & 2 & 14 & 12.5 & 476 & 425 & 4500 & 1 & 7 & $v$ & 3 & \\
\hline 94 & 5 & 3 & 87 & & & & 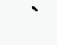 & $\mathbf{M H}$ & 180 & 65000 & 2 & 4.5 & 3.8 & $\mathbf{4 4 0}$ & 372 & 5400 & 1 & 7 & $\mathbf{u}$ & 3 & \\
\hline 17 & 5 & 10 & 88 & & & & & $C T$ & 2300 & 624000 . & 2 & 90 & $68: 5$ & 620 & 472 & 5500 & $i$ & 7 & $\mathbf{u}$ & 3 & \\
\hline 157 & 2 & & . & 9 & 93 & & & RI & 641 & 234000 & 2 & 21 & 17 & 671 & .543 & 5200 & 1 & 7 & $\mathbf{u}$ & 3 & \\
\hline 19 & 3 & & & 10 & 91 & & & $C T$ & 522 & 190530 & 2 & 18 & 16 & 585 & 520 & 5000 & 1 & 7 & $\mathbf{u}$ & 3 & \\
\hline 18 & 2 & & & 3 & 93. & - & & $\boldsymbol{C r}$ & 425 & 131750 & 2 & 15 & 13 & 600 & 550 & 4500 & 1 & 7 & $\mathbf{u}$ & 3 & \\
\hline 16 & 2 & & & 3 & 94 & & & ${ }^{\circ} \mathrm{CT}$ & 468 & 170638 & 2 & 14.5 & 12 & 677 & 560 & 5300 & -1 & 7 & $\mathbf{u}$ & 3 & - \\
\hline 55 & 5 & 9. & 88 & & & & & $\mathbf{M E}$. & 500 & 163000 & 2 & 13.6 & 10 & 500 & 368 & 5000 & .1 & 7 & $\mathbf{u}$ & 3 & \\
\hline 14 & 5 & 7 & 88 & . & & & & $C T$ & 2000 & 720000 & 2 & 67 & 60 & 720 & 640 & 5300 & 1 & 7 & u & 3 & $\cdots$ \\
\hline 186 & 8 & 1 & 88 & 7 & 91 & 8 & 88 & VT & 204 & 75000 & 2 & 7 & 6 & 548. & 470 & 5253 & 1 . & 5 & $u$ & 3 & \\
\hline .15 & 5 & 3 & 88 & & - & - & & $C T$ & 600 & 195000 & 2 & 16 & 13.5 & $620^{\circ}$ & .535 & 5000 & 1 & 7 & $\mathbf{u}$ & 3 & \\
\hline 52 & 3 & & & 7 & 92 & & & ME & 176 & 64240 & 2 & 5 & 3.8 & 620 & .471 & 5200 & .1 & $7^{\prime}$ & $u$ & 3 & \\
\hline 53 & 5 & 12 & 87 & & & & & $\mathrm{ME}$ & 607 & 221555 & 2 & 22 & 20 & 620 & $564^{\circ}$ & 6200 & 1 & 7 & $\mathbf{u}$ & 3 & \\
\hline 54 & 5 & 6 & 88 & & & & . & $\cdot \mathbf{M E}$ & 750 & 215000 & 2 & 25.3 & 21 & 545 & 455 & 6200 & 1 & 7 & $\mathbf{u}$ & 3 & · \\
\hline 109 & .5 & 5 & 90 & & & & & NJ & -525 & 165000 & 2 & 14 & 12. & .475 & 425 & 4500 & 2 & 3 & $\mathbf{v}$ & 3 & \\
\hline 107 & 2 & . & & 10 & 94 & & . & NT & .1166 & 425517 & 2 & 40 & 34 & 535 & 455 & 5500 & 2 & 3 & $\mathbf{u}$ & 3 & \\
\hline 104 & 3 & & & & 94 & & & NN & 425 & 156000 & 2 & 12.5 & 9.8 & 542 & 425 & 5200 & 2 & 3 & $\mathbf{u}$ & 3 & \\
\hline 105. & 2 & & & 1 & $93^{\circ}$ & & & NT & 1200 & 437000 & 2 & 44 & 39 & 670 & 567 & .5400 & 2 & 3 & $\mathbf{u}$ & 3 & \\
\hline 106 & 2 & & & & 95 & & & SW & 2400 & 876000 & 2 & 88 & 80 & 530 & .482 & 4500 & 2 & 3 & $\mathbf{u}$ & 3 & \\
\hline 108 & 2 & & & 7 & 95 & & & ลง & 1445 & 527425 & .2 & 63 & 57 & 620 & 561 & 4950 & 2 & 3 & $\mathbf{u}$ & 3 & \\
\hline 101 & 3 & & & 10 & 90 & & & WN. & 2000 & 725000 & 2 & $76^{\circ}$ & 72. & 501 & 475 & 4500 & 2 & 3 & $\mathbf{u}$ & 3 & \\
\hline 103. & .2 & 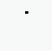 & & .9 & 93 & & & $\cdot \mathrm{N}$ & 1219 & 400000 & 2 & 45 & $37: 3$ & 753 & 625 & .5500 & 2 & 3 & $\mathbf{u}$ & 3. & \\
\hline 102 & .5 & 7 & 88 & & & & & $\mathrm{NWT}$ & 450 & 124100 & 2 & $13.5^{\circ}$ & 10.5 & 620 & 482 & 4650 & 2 & 3 & $u$ & 3 & $\cdot$ \\
\hline $146^{\circ}$ & 3 & & & 69 & & & & EA & 2285 & 834000 & 2 & 90 & 80 & 675 & 600 & 5200 & 2 & 3 & $\mathbf{u}$ & 3 & \\
\hline
\end{tabular}

54 - Energy Information Administration/NEMS Renewable Fuels Module Documentation Report-MSW . 
- Table 2E-2. Plant-Level Data with Missing Valueś Filled In and Sector Code Added (Continued)

\begin{tabular}{|c|c|c|c|c|c|c|c|c|c|c|c|c|c|c|c|c|c|c|c|c|c|c|}
\hline 1 & 2 & 3 & 4 & 5 & 6 & 7 & $8^{\circ}$ & 9 & 10 & 11 & 12 & 13 & 14 & .15 & $16 .-$ & 17 & .18 & 19 & 20 & 21 & & \\
\hline 147 & 3 & & & 5 & 91 & & & PA & 1080 & 330000 & 2 & 36 & 30 & 672 & 560 & 5000 & 2 & 3 & u & 3 & & \\
\hline 145 & 2 & & & 7 & 93 & & & PA & 2250 & 700000 & 2 & 72 & . 60 & 620 & 517 & 5253 & 2 & 3 & $\mathbf{u}$ & 3 & & \\
\hline 127 & 2 & & . & & 95 & & & NY & 900 & 328500 & 2 & 21 & 17 & 620 & 502 & 5253 & 2 & 6 & $\mathbf{u}$ & 3 & & \\
\hline 132 & 5 & 5 & 90 & & & & & $\mathrm{NY}$ & 2505 & 914000 & 2 & 72 & 64 & 641 & 570 & 4500 & 2 & 6 & $\mathbf{u}$ & 3 & & \\
\hline 154 & 3 & & & 1 & 92 & & & PA & 1200 & 277000 & 2 & 34 & 29 & 460 & 392 & 4500 & 2 & 3 & $\mathbf{u}$ & 3 & & \\
\hline 155 & 2 & & & 7 & 93 & & & $\mathrm{PA}$ & 1275 & 465375 & 2 & 45 & 40 & 620 & 551 & .5200 & 2. & 3 & $\mathbf{u}$ & 3 & & \\
\hline 153 & 5 & 11 & 89 & & & & & EA & 1100 & 378000 & 2 & 35 & 30.2 & 626 & 540 & 4500 & 2 & 1 & $\mathbf{u}$ & $\dot{3}$ & & \\
\hline 149 & 2 & & & 1 & 93 & & & PA & 1925 & 700000 & 2 & 72 & 65 & 665 & 600 & 5200 & 2 & 3 & $\mathbf{u}$ & 3 & & \\
\hline 150 & 2 & & & 1 & 94 & & & PA & 425 & 155000 & 2 & 14 & 12.5 & 588 & 525 & 5200 & 2 & 3 & $\mathbf{u}$ & 3 & & \\
\hline 119 & 3 & & & 10 & 91 & & & NYY & 345 & 126000 & 2 & 13. & 11 & 620 & 525 & 5500 & 2 & 6 & $\mathbf{u}$ & 3 & & \\
\hline 120. & 5 & 6 & 89 & & & & & STY & 450 & 160000 & 2 & 11.5 & 8 & 532 & 370 & 4450 & 2 & 6 & $u$ & 3 & $\cdot$ & \\
\hline $117^{\circ}$ & .3 & & & $3^{\circ}$ & 92 & & & $\mathrm{NY}$ & 638 & 253000 & 2 & 25 & 21 & 736 & 627 & 6000 & 2 & 6 & $\mathbf{u}$ & 3 & & \\
\hline 113 & 5 & 4 & 89 & & & & & $N Y$ & $64 \dot{0}$ & 210000 & 2 & 17 & 14 & 498 & 410 & 5000 & 2 & 6 & $\mathbf{u}$ & 3 & & \\
\hline 114 & .2 & & & 1 & 94 & & & $N Y$ & 1275 & 465000 & 2 & 50 & 40 & 620 & 496 & 5500 & 2 & 6 & $\mathbf{u}$ & 3 & & \\
\hline 125 & 2 & & & 10 & 93 & & & NY & 850 & 310000 & 2 & 38 & 32 & 760 & 640 & 6000 & 2 & $6^{\circ}$ & $\mathbf{u}$ & 3 . & & \\
\hline 126 & 5 & 10 & 84 & & & & . & $\mathbf{N Y}$ & 1890 & 657000 & i & 60 & 55.5 & $638^{\circ}$ & 590 & 4800 & 2 & $6^{\circ}$ &.$u^{\circ}$ & 3 & & \\
\hline 124 & 2 & & & 10 & 93 & & & NY & 850 & 300000 & 2 & 31 & 27 & -620 & 540 & 6000 & 2 & 6 & $\mathbf{u}$ & 3 & & \\
\hline 121 & 2 & . & & 2 & 93 & & $\therefore$ & $N Y$ & 485 & $165000^{\circ}$ & 2 & 18 & 15 & 560 & 467 & 5200 & 2 & 6 & $\mathbf{u}$ & 3 & & \\
\hline 122 & 5 & $\cdot 2$ & 88. & - & & & & N̦Y & 170 & 62050. & 2 & 4.5 & 3 & 620 & 413 & 5000 & 2 & 6 & u & 3 & & \\
\hline 98 & 3 & & & 3 & 91 & & & NT & 840 & 306600 & 2 & 30 & 21. & 689 & $482^{\circ}$ & .4500 & 2 & 3 & $\mathbf{u}$ & 3 & & \\
\hline 99 & 2 & & & 10 & 93 & & & $\mathrm{NWT}$ & 830 & 302500 & 2 & 36 & . 32 & 655 & 560 & 5000 & 2 & 3 & $\mathbf{u}$ & 3 & & \\
\hline 100 & 2 & & & & 93 & & & มก' & 1265 & 438000 & 2 & 45 & 38.25 & 535 & 455 & 4500 & 2 & $3 \cdot$ & $\mathbf{u}$ & 3 & & \\
\hline 138 & 5 & & & & - & & & $\mathrm{OH}$ & 1600 & 584000 & 2 & 37 & 32 & 568 & 491 & 4800 & 3 & 1 & $\mathbf{u}$ & 3 & & \\
\hline 139 & 5 & 8 & 88 & & & . & & OH & 255 & $\therefore 93075$ & 2 & 6 & 5.7 & 550 & 523 & 5000 & 3 & 1 & u & 3 & & \\
\hline $74^{\circ}$ & 2 & & , & $\therefore$ & 94 & & & III & 476 & 173740 & 2 & 13 & 11. & 620 & 525 & 6000 & 3 & 1 & $\mathbf{u}$ & 3 & & \\
\hline 69 & 2 & 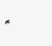 & & 1 & 94 & & & MII & 1700 & 620500 & 2 & 62 & 54 & $74 i$ & 645 & 5200 & 3 & 1 & $\boldsymbol{u}$ & 3 & & \\
\hline 140 & 2 & & & & 93 & & & OH & 810 & 225000 & -2 & 18.6 & 17.7 & 507 & 482 & 5000 & 3 & 1 & $\mathbf{u}$ & 3 & & \\
\hline 200 & 2 & & & • & 93 & & & WI & 128 & $46500^{\circ}$ & 2 & 2.75 & 2.5 & 323 & 263 & 5000 & 3 & 4 & $\mathbf{u}$ & 3 & & \\
\hline 197 & 5 & 1 & 79 & & & & & WI & 250 & 650001 & 2 & 100 & 86.88 & 620 & 539 & 5759 & 3 & 4 & $\mathbf{u}$ & 3 & & \\
\hline 196 & 5 & 7 & $88^{-}$ & & & & & wI & 225 & 56000 & 2 & 30 & $28^{\circ}$ & 620 & 579 & 5500 & 3 & 4 & u & 3 & & \\
\hline 46 & 2 & . & & 1 & 94 & & $\therefore$ & $I I$ & 1020 & 379746 & 2 & 50 & 41 & 976 & 800 & $6100^{\circ}$ & 3 & 4 & $u_{. .}$ & 3 & & \\
\hline 50 & 5 & 9 & 75 & & & & & IA & 174 & 43500 & 2 & 100 & 95 & 620 & 589 & 6200 & 4 & 5 & $u$ & 3 & & \\
\hline 82 & 5 & 3 & 90 & & · & & & $\mathbf{M N}$ & 1000 & 365000 & 2 & 37.5 & 33 & 700 & 540 & 5800 & 4 & 5 & $u$ & 3 & & . \\
\hline 79 & 5 & .8 & 89 & & & & & $\mathbf{M N}$ & 1500 & 460500 & 2 & 35 & -30.41 & 700 & 608 & $\overline{5500}$ & 4 & 5 & u & 3 & & \\
\hline 87 & 2 & & & 3 & 93 & & & MN & 640 & 233600 & 2 & 23. & $20^{\circ}$ & 633 & 550 & 5000 & 4 & 5 & $\mathbf{u}$ & 3 & & $\because$ \\
\hline 83 & 5 & $?$ & 87 & & & & & MNS & 1175 & 400000 & 2 & 22 & 19.5 & 620 & 550 & 5500 & 4 & 5 & $\mathbf{u}$ & 3 & & \\
\hline 28 & 5 & 7 & 83 & & & & & EL & 275 & 63250 & 2 & 364 & 335 & 620 & 571 & 4500 & 5 & 8 & $\mathbf{u}$ & 3 & & \\
\hline 25 & 3 & & & 1 & 92 & 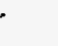 & & EL & 2250 & 821250 & 2 & 63.4 & 57 & .676 & 608 & 5200 & 5 & 8 & $\mathbf{u}$ & 3 & & \\
\hline 24 & 5 & 10 & 87. & & & - & & FL & 1200 & 430,000 & 2. & 30 & 27.5 & 537 & 492 & 4500 . & 5 & 8 & $u$ & 3 & & \\
\hline $27^{\circ}$ & 5 & 12 & 86 & & & & & FL. & 130 & 46538 & 2 & 2.6 & 2.2 & 355 & 300 & 5000 & 5 & 8 & $\mathbf{u}$ & 3 & . & \\
\hline 135 & 2 & & & 6 & .93 & & & NC & 400 & 140400 & 2 & 6.8 & 5.5 & 680 & 550 & 5253 & 5 & 9. & $\mathbf{u}$ & 3 & & \\
\hline
\end{tabular}


Table 2E-2. Plant-Level Data with Missing Values Filled In and Sector Code Added (Continued)

\begin{tabular}{|c|c|c|c|c|c|c|c|c|c|c|c|c|c|c|c|c|c|c|c|c|c|c|}
\hline 1 & 2 & 3 & 4 & 5 & 6 & 7 & 8 & 9 & 10. & 11 & $12^{\circ}$ & 13 & 14 & 15 & 16. & 17 & .18 & 19 & 20 & 21 & & \\
\hline 38 & 5 & 11 & 89 & & • & & & FL & 2000 & 624000 & 2 & 61 & .49 & 600 & 500 & 4865 & 5 & 8 & $u$ & 3 & & \\
\hline 34 & 5 & 6 & 87 & & & & & FI & 435 & 186642 & 2 & 12 & 10 & 480 & 432 & 4600 & 5 & 8 & $u$ & 3 & & \\
\hline 36 & 5 & 5 & 83 & & & & & FI & 2835 & 920000 & 2 & 62 & 55.8 & 478 & 430 & 4000 & 5 & 8 & u & 3 & & \\
\hline 37 & 3 & & & .1 & 92 & & & FI & 2250 & 821250 & 2 & 66.5 & 60 & 709 & 638 & 5200 & 5 & 8 & u & 3 & & \\
\hline 39 & 3 & & & 7 & 91 & & & FL & 893 & 325945 & 2 & 31 & 29 & 650 & 550 & 4800 . & 5 & 8 & u & .3 & & \\
\hline 31 & 5 & 1 & 82 & & & & & FL & 2800 & 1022000 & 2 & 77 & 62 & 480 & 388 & 5000 & 5 & 8 & u & 3 & & \\
\hline 29 & 2 & & & $i$ & 94 & & & $F I$ & 1530 & 558450 & 2 & 50 & 47 & 670 & 630 & 5000 & 5 & 8 & u & 3 & & \\
\hline 40 & 5 & 9 & 85 & & & & & FL. & 844 & 306000 & 2 & 17 & 15 & 510 & 450 & 5000 & 5 & 8 & $u$ & 3 & & \\
\hline 33 & 4 & & & 3 & 91 & & & FL & 449 & $163000^{\circ}$ & 2 & 14.5 ? & 10 & 525 & 362. & 5000 & 5 & 8 & u & 3 & & \\
\hline 57 & 6 & 1 & .76 & & & & & $M$ & 600 & 186000 & 2 & 200 & 188 & 620 & 583 & 6800 & 5 & 3 & u & 3 & & \\
\hline 181 & .5 & 6 & 90 & . & & & & $\mathrm{VA}$ & 2700 & 985150 & 2 & $85^{\circ}$ & $73^{\circ}$ & 610 & 540 & 4400 & 5 & 1 & u & 3 & & \\
\hline 58 & 2 & & $-\cdot$ & 3 & 94 & & & $\mathbb{M D}$ & 1530 & 558450 & 2 & 83.6 & 69 & 780 & 644 & 5500 & 5 & 3 & $\mathbf{u}$. & 3 & & \\
\hline 183 & 2 & - & & & 93 & & & VA & 700 & 182000 & 2 & 12 & 9 & 620 & 465 & 5253 & 5 & 9 & u & 3 & & \\
\hline 177 & 5 & 2 & 88 & & & & & VA & 940 & 338400 & 2 & 22 & 19.8 & 520 & 470 & 4800 & 5 & 1 & u & 3 & & \\
\hline 172 & 5 & 3 & 86 & & . & & & $\mathrm{TX}$ & 85 & 20400 & 2 & 0.86 & 0.66 & 620 & 476 & 4500 & 7 & 2 & $u$ & 3 & & - \\
\hline 142 & 8 & & 85 & 1 & 93 & 9 & 86. & or. & 700 & 300000 & 2 & 21.8 & 10 & 620. & 284 & 5200 & 7 & 10 & u & 3 & & \\
\hline 9 & 5 & 5 & 87 & & & & & $C A$ & 380 & 110000 & 2 & 11.5 & 10 & 725 & 630 & 5600 & 9 & 13 & u & 3 & & \\
\hline 187 & 5 & 11 & 86 & . & & & & $W A$ & 100 & 35000 & 2 & 1.5 & 1 & 525 & 350 & 4500 & 9 & 11 & $\mathbf{u}$ & 3 & & \\
\hline 42 & 5 & 5 & 90 & & & . & - & HI & 1740 & 600000 & 2 & 55 & 46 & 550 & $460^{\circ}$ & 4800 & 9 & 13 & $\mathbf{u}$ & 3 & & \\
\hline 191 & 3 & & : & 8 & 91 & & & WA & 680 & 248200 & 2 & 26 & 22.1 & 585 & 497 & 5253 & 9 & 11 & u & 3 & & \\
\hline 10 & 5 & 1 & 89 & & & & & $C A$ & 730 & 293000 & 2 & 22.5 & 17. & .596 & 450 & 4750 & 9 & 13 & $\mathbf{u}$ & 3 & & \\
\hline 144 & 5 & 5 & 86 & & & & v & OR & 535 & 190000 & 2 & 13.1 & 11 & 536 & 450 & 4700 & 9 & 11 & u & 3 & & \\
\hline 190 & 5 & 7 & 88 & & & & & WA & 150 & 54000 & 2 & 2.4 & $2: 04$ & 406 & 345 & 4500 & 9 & 11 & u. & 3 & & ; \\
\hline 11 & 4 & 12 & 88 & & • & & & $C A$ & 1170 & 427000 & 2 & 36 & 30 & 648 & 540 & $4800^{\prime}$ & 9 & 13 & u. & 3 & & \\
\hline 193 & 5 & 3 & 90 & & & & & $\cdot \mathrm{WA}$ & 250 & 100000 & 2 & 50. & 43 & 620 & 533 & 5600 & 9 & 11 & $\mathrm{u}$ & 3 & & \\
\hline 189 & 2 & & & & 95 & & & WAA & 2200 & 803000 . & 2 & 90 & 77 & 660 & 565 & 5000 & 9 & 11. & $\mathbf{u}$ & 3 & & \\
\hline 12 & 2 & & & 10 & 94 & & & $C A$ & 1822 & 638000 & $2^{\circ}$ & 41 & 36 & 615 & 540 & 4900 & 9 & 13 & $\mathbf{u}$ & 3 & & \\
\hline 21 & 5 & $1 i$ & .81 & & & & $\because$ & $\mathrm{Cr}$ & 130 & 37000 & 3 & 2.2 & 1.9 & $150^{\circ}$ & 130 & .5000 & 1 & 7 & $\mathbf{u}$ & 3 & & \\
\hline 20 & 5 & 5 & 89 & & & . & & $C T$ & 380 & 138000 & 3 & 11 & 9.3 & 500 & 384 & 4850 & 1 & 7 & ui & 6 & & \\
\hline 60 & 5 & 8 & 88 & 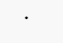 & 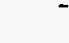 & & & MA & 324 & 112500 & $3^{\circ}$ & 8.6 & 7.1 & 472 & 390 & 4200 & 1 & 7 & $\mathbf{u}$ & 3 & & \\
\hline 63 & 5 & $\overline{3}$ & 85 & & & & & $\mathbf{M A}$ & 610 & 222000 & 3 & 21 & 17 & 505 & 409 & 6000 & 1 & 7 & ui & 6. & & \\
\hline 158 & 2. & & - & 4 & 93 & & & $\mathbf{R I}$ & .645 & 238000 & 3 & 21 & 18 & 531 & 455 & 4750 & 1 & 7 & ui & 6 & & \\
\hline 130 & 5 & 1 & 85 & : & $\cdot$ & & & $\sqrt[3]{Y}$ & 160 & 55000 & 3 & 2.2 & 1.2 & 505 & 275 & 5253 & 2 & 6 & ui & 6 & & \\
\hline 128 & 5 & 10 & 88 & & & & 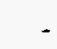 & $\sqrt{x} x$ & 400 & 120000 & 3. & 10 & 9.2 & 320 & 140 & 5253 & 2 & 6 & vi & 6. & & \\
\hline .118 & .5 & 8 . & 83 & & & & & $2 \sqrt{Y}$ & 225 & - 78750 & 3 & $2.5^{n}$ & 1 & 505 & 202 & 5000. & 2 & 6 & $u i$ & 6 & & \\
\hline 123 & 5. & 12 & 80 & $\therefore$ & . & & & WY & 1800 & 583900 & 3. & 50 & 30. & $505^{\prime}$ & 303 & $5253^{\circ}$ & 2. & 6 & $u i$ & 6 & & \\
\hline 151 & 5 & 10 & 72. & & & & - & PA & 620 & 200000 & 3 & 8.2 & 5.25 & 781 & 500 & 4500 & 2 & 1 & ui & 6 & & \\
\hline 131 & 5 & 2 & 86 & & & & & $\sqrt{Y}$ & 190 & 、70000 & 3 & 3.6 & 1 & 990 & 275 & 5000 & 2 & 6 & ui & .6 & & \\
\hline .148 & 2 & 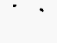 & & -1. & 92. & $\therefore$ & & $\mathrm{PA}^{\circ}$ & 63 & 21716 & 3 & 0.75 & 0.35 & 275 & 130 & 4500 & 2 & 3 & $i$ & 2 & & \\
\hline .198 & 5 & 5 & 89 & . & & & & WI & 80 & $22880^{\circ}$ & 3 & 1.4 & 0.8 & 150 & 100 & $\cdot 5450$ & $3^{i}$ & 4 & ui & .6 & & \\
\hline 195 & 5 & 10 & $\cdot 86$ & & & & & WI & 80 & 29000 & 3. & 0.27 & 0.07 & 505 & 131 & 4750 & 3 & 5 & ui & 6 & & \\
\hline
\end{tabular}

56 - Energy Information.Administration/NEMS Renewable Fuels Module Documentation Report-MSW 
Table 2E-2. Plant-Level Data with Missing Values Filled In and Sector Code Added (Continued)

\begin{tabular}{|c|c|c|c|c|c|c|c|c|c|c|c|c|c|c|c|c|c|c|c|c|c|}
\hline 1 & 2 & 3 & 4 & 5 & 6 & 7 & 8 & 9 & 10 & 11 & 12 & 13 & 14 & 15 & 16. & $\therefore \quad 17$ & .18 & 19 & 20 & 21 & \\
\hline 47 & 2 & & & 7 & 95 & & & IN & 367 & 113867 & 3 & 10.5 & 8.9 & 505 & 428 & 5253 & 3 & 1 & $\mathbf{u}$ & 3 & \\
\hline 73 & 2 & & 93 & & & & 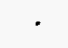 & MII & 150 & . 54750 & 3 & 2.8 & 2.3 & .454 & 373 & 5253 & 3 & $i$ & ui & 6 & \\
\hline 72 & 5 & 10 & 87. & & & . & • & MII & 200 & 70000 & 3 & 2 & 1.7 & 505 & 429 & 4900 & 3 & 1 & c & 1 & \\
\hline 71 & $5^{\circ}$ & 1 & 90 & & & & & MII & .625 & 194000 & 3 & 18.3 & 15.7 & 478 & 410 & 5350 & 3 & $\frac{1}{2}$ & ue & 5 & \\
\hline 45 & 2 & & & & · & & - & $I x$ & 380 & 144000 & 3 & 8 & 6 & 507 & 380 & 5253 & 3 & 4 & $\mathbf{u}$ & 3 & \\
\hline 202. & 2 & . & * & 1 & 94 & & & $w I$ & 510 & 219000 & 3 & $16.5^{\circ}$ & 12.19 & 505 & 374 & 5500 & 3 & 4 & $\mathbf{u}$ & 3 & \\
\hline 44 & $\ddot{5}$ & 9 & 70 & & & & & IL & 1250 & 400000 & 3 & -30.1 & 22.26 & 505 & 374 & 5253 & 3 & 4 & ui & 6 & \\
\hline .70 & 5 & & 89 & & & & & MII & 2900 & 754000 & 3 & 65 & 48.11 & 505 & 374 & 5253 & 3 & 1 & ue & 5 & \\
\hline 137 & 5 & & 79 & & & & & $\mathrm{OH}$ & 965 & 265000 & 3 & 4 & 2.961 & 505 & 0 & 4800 & 3 & 1 & c & 1 & \\
\hline 199 & 5 & & 89 & & & - & & WI & $105^{\circ}$ & 33600 & 3 . & 1.2 & 0.7 & 110 & 85 & 5000 & 3 & 5 & ue & 5 & \\
\hline 86 & 5 & & 87 & & & & & $\mathbf{M N}$ & 190 & 59000 & 3 & 3 & 2.25 & 293 & 220 & $\mathbf{5 5 0 0}$ & 4 & 5 & 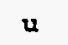 & 3 & \\
\hline 184. & 5 & & 88 & & & & & $\mathrm{VA}$ & 1400 & 364000 & 3. & 40 & 35 & 505 & 442 & 5550 & 5 & 9 & $i$ & 2 & \\
\hline 56 & 5 & 5 & 85 & & & & & $\mathbf{2 D}$ & 1947 & 710500 & 3 & 60 & 34 & 400 & 350 & 5100 & 5 & 3 & $\mathbf{u}$ & 3 & \\
\hline 134 & 2 & & & & 92 & & & NC & 300 & 106500 & 3 & 5 & 4.25 & 505 & 429 & 5253 & 5 & 9 & ui & 6 & \\
\hline 136 & 7 & 6 & 84 & & & & & $\mathrm{NC}$ & 85 & 51000 & 3 & 4 & 2 & 505 & 252 & 5253 & 5 & 9 & ui & 6 & \\
\hline 160 & 5 & 11 & 89 & & & & & sc & 600 & 210000 & 3 . & 12.8 & 10.8 & 505 & 426 & 5000 & 5 & 9 & ui & 6 & - \\
\hline 133 & 5 & 6 & 89 & & & & & NC & 195 & 71000 & 3 & 5.3 & 4 & 476 & 395 & $4500^{\circ}$ & 5 & 9 & ue & 5 & \\
\hline 23 & 5 & 11. & .87 & & & -. & - & $\mathrm{DE}$ & .546 & 200000 & 3 & 13.3 & 10.5 & 674 & 532 & 5500 & 5 & 3 & ui & 6 & \\
\hline 166 & 3 & & & 12 & 92 & & & $\mathrm{TN}$ & 205 & 75000 & 3 & $\cdot 4$ & 3.25 & 505 & $410^{\circ}$ & 6000 & 6 & 9 & $u i$ & 6 & - \\
\hline 163 & 5 & 12 & 81 & & & & & $T N$ & 180 & 60000 & .3 & 0.5 & 0.43 & 505 & 434 & 5253 & 6 & 9 & ui & 6 & \\
\hline $167 !$ & 5 & 2 & 74 & & & & & $T N$ & 950 & 346750 & 3 & 7.3 & 2.9 & 505 & 200 & 4900 & 6 & .9 & uc & 5 & \\
\hline 143 & .5 & 3 & $86^{\circ}$ & & & & & OK & 1060 & 346960 & $3:$ & 16.5 & 14.5 & 600 & 530 & 5000 & 7 & 10 & $\mathbf{u}$ & 3 & \\
\hline 93 & 2 & & & 1 & 95 & & & $\mathrm{NV}$ & 712 & 260000 & 3 & 50 & 40 & 505 & 404 & 5253 & 8 & 11 & $\mathbf{u}$ & 3 & \\
\hline 13 . & 8 & 11 & 84 & 7 & 91 & 5 & 85 & $\mathrm{CA}$. & 100 & 36500 & 3 & 1.54 & 1.2. & 505 & 393 & 6500 & 9 & $13^{\circ}$ & ue & 5 & \\
\hline 111 & 5 & 2 & 81 & & $\cdot$ & . & & $M Y$ & 720 & 148000 & 4 & 0 & 0 & 0 & 0 & 5253 & 2 & 6 & c & 1. & \\
\hline $49^{\circ}$ & 5 & 9 & $90^{\circ}$ & . & & & & $I N$ & 125 & 32500 & 4 & 0 & 0 & 0 & 0 & 5253 & 3 & 1 & $i$ & 2 & \\
\hline 161 & 5 & 12 & 89. & & & - & . & SD & 65 & 16900 & 4 & 0 & 0 & 0 & 0 & 5253 & 4 & 5 & c & .2 & \\
\hline 89. & 5 & 11 & 85 & & & & & .207 & 50 & 10000 & 4 & 0 & 0. & 0 & 0 & 5253 & 4 & 5 & c & 1 & \\
\hline 51 & 5 & 10 & 88 & • & & & & $I A$ & 100 & 25500 & $\cdot 4$ & 0 & 0 & ? & 0 & 5253 & 4 & 5 & $i$ & 2 & \\
\hline 78 & 5 & 3 & 87 & & & & & MNT & 470 & 120000 & 4 & 0 & 0 & 0 & 0 & 5253 & 4 & 5 & ui & 6. & \\
\hline 26 & 8 & 4 & 89 & & & 9 & 89 & FL & 540 & 140400 & 4 & 0 . & .0 & 0 & 0 & 5253 & 5 & 8. & $i$ & 2 & \\
\hline 35 & 3 & & & 1 & 92. & $\cdots$ & & FL & 660 & 207000 & 4 & 0 & 0 & 0 & 0 & 5253 & 5 & 8 & i & 2 & $\because$ \\
\hline 194 & 3 & 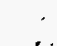 & & & 91 & & & WV & 550 & 132000 & 4 & 0 & 0 & 0 & 0 & 5253 & 5 & 1 & $i$ & 2 & \\
\hline 22 & 5 & 3 & 84 & & & & & $\mathrm{DE}$ & 600 & 230000 & 4 & 0 & 0 & 0 & 0 & 5253 & 5 & 3 & $u$ & .3 & \\
\hline 165 & 5 & 10 & $89^{\circ}$ & & . & & & $T N$ & 48 & 12480 & 4 & 0 & 0 & 0 & 0 & 8000 & 6 & 9 & $i$ & 2 & \\
\hline 168 & 4 & 10 & 90 & & & & & TN & 50 & 13000 & 4 & 0 & 0 & 0 & 0 & .5253 & 6. & 9. & $i$ & 2 & \\
\hline 5 & .4 & & - & $9^{\circ}$ & 90 & . & & $\mathrm{AK}$ & 200 & $60000^{\circ}$ & 4. & 0 & 0 & 0 & 0 & 5253 & 9 & 11 & c & 1 & \\
\hline 192 & $5^{\prime}$ & .7 & 79 & & & & & WA. & $450^{\circ}$ & 127000 & .4 & $: 0$ & 0 & 0 & 0. & 5253 & 9 & .11 & $\mathbf{u}$ & 3 & \\
\hline 4 & 5 & 6 & 81. & & & & & $\mathrm{AR}$ & 45 & 15435 & 5 & 0 & .0 & 0 & ? & 5253 & 9 & 11 & c & 1 & \\
\hline
\end{tabular}


Table 2E-3 shows the reduced the data set, containing only those numbers that are used to calculate $F, H R 1$, and $M A P C R N R$. A plant. in Puerto Rico was eliminated from the data set because Puerto Rico is not modeled in NEMS. The first two columins are the plant number and State, which are used for identification purposes only. The rest of the column headings are the same as the variable names used in the FORTRAN data procesșing program. The variable names are:

$$
\begin{aligned}
& \text { QMSW = quantity of MSW in tons/year utilized at the facility } \\
& \text { OUTTYP = type of energy output, . } \\
& 1 \text { = steam only } \\
& 2=\text { electricity only } \\
& 3=\text { steam and electricity }
\end{aligned}
$$

$$
\begin{aligned}
& 1=\text { commercial } \\
& 2=\text { industrial } \\
& 3=\text { utility }
\end{aligned}
$$


Table 2E-3. Data Set Used as. Input to FORTRAN Program that calculates $F, H R$, and MAPCRNR

QMSW OUTTYP GR KWH NT KWH BTU CR NR SNO

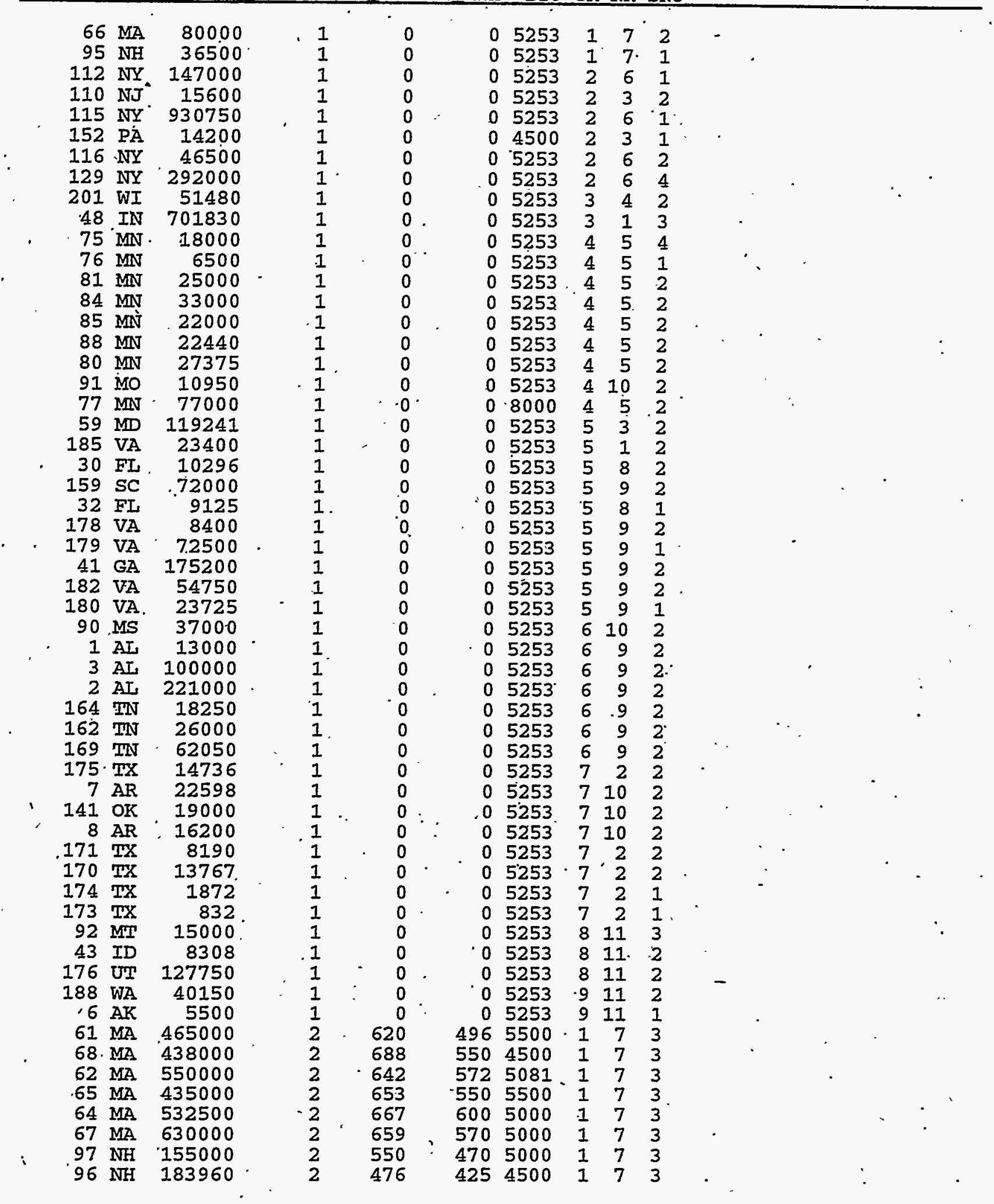


Table 2E-3. Data Set Used as Input to FORTRAN Program that calculates $F, H R$, and MAPCRNR (Continued)

QMSW OUTMYP GR KWH NT KWH BTU'CR NR SNO

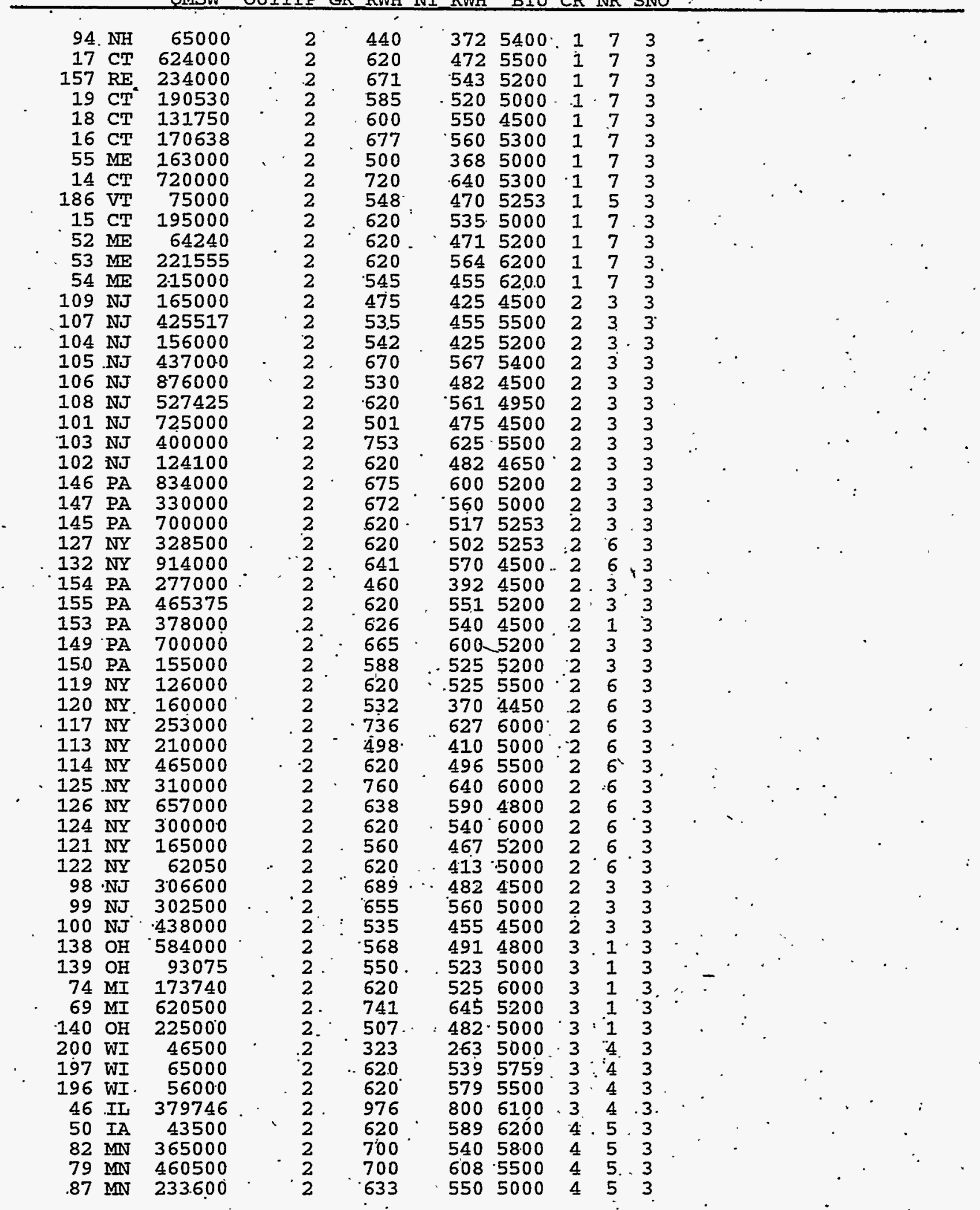


Table 2E-3. Data Set Used as Input to FORTRAN Program that calculates $F, H R$, and MAPCRNR (Continued)

QMSW OUTTYP GR KWH NT KWH BTU CR NR SNO

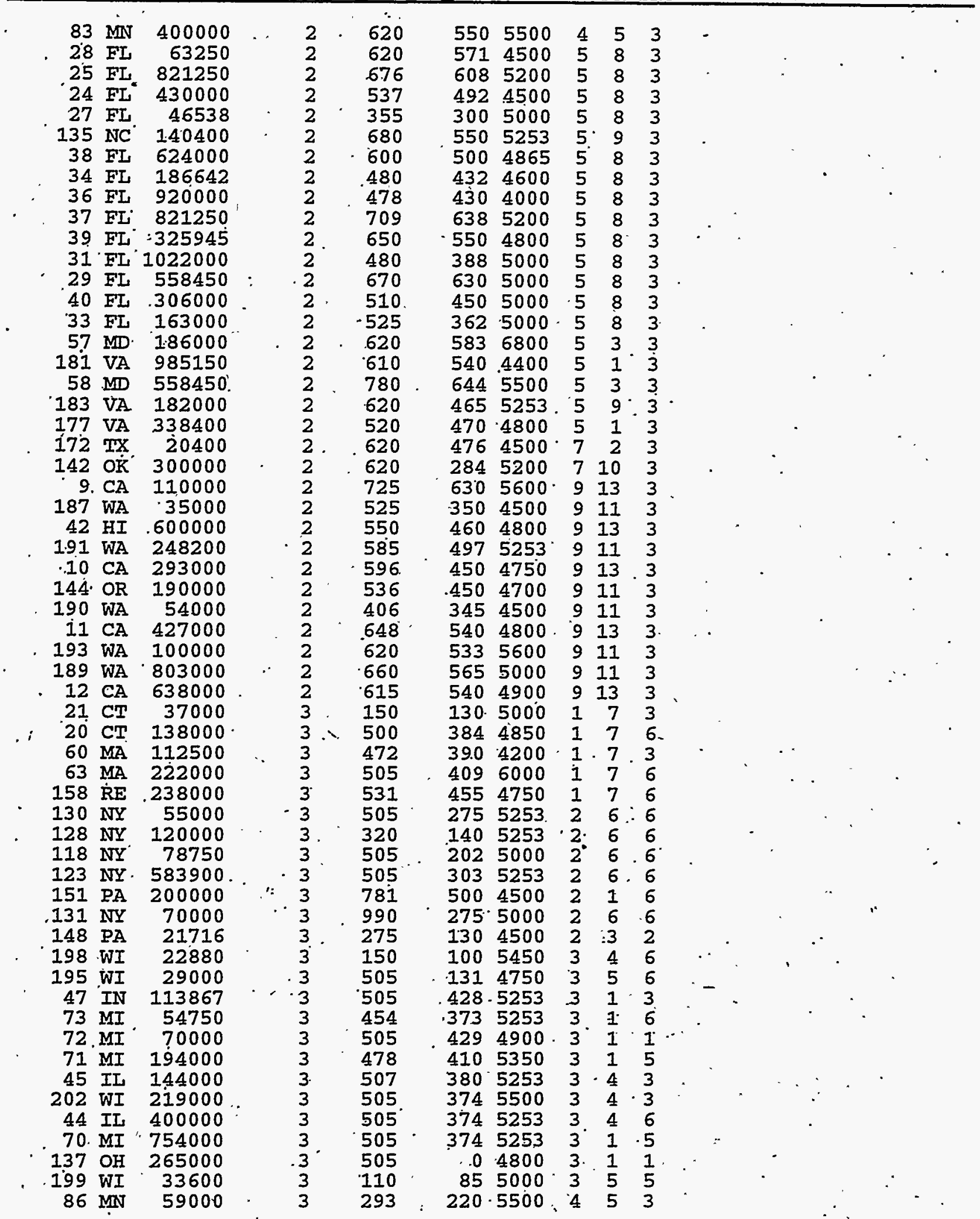


Table 2E-3. Data Set Used as Input to F.ORTRAN Program that calculates $F, H R$, and MAPCRNR (Continued)

OMSW OUTMYP GR KWH NT KWH BTU CR NR SNO $\because$

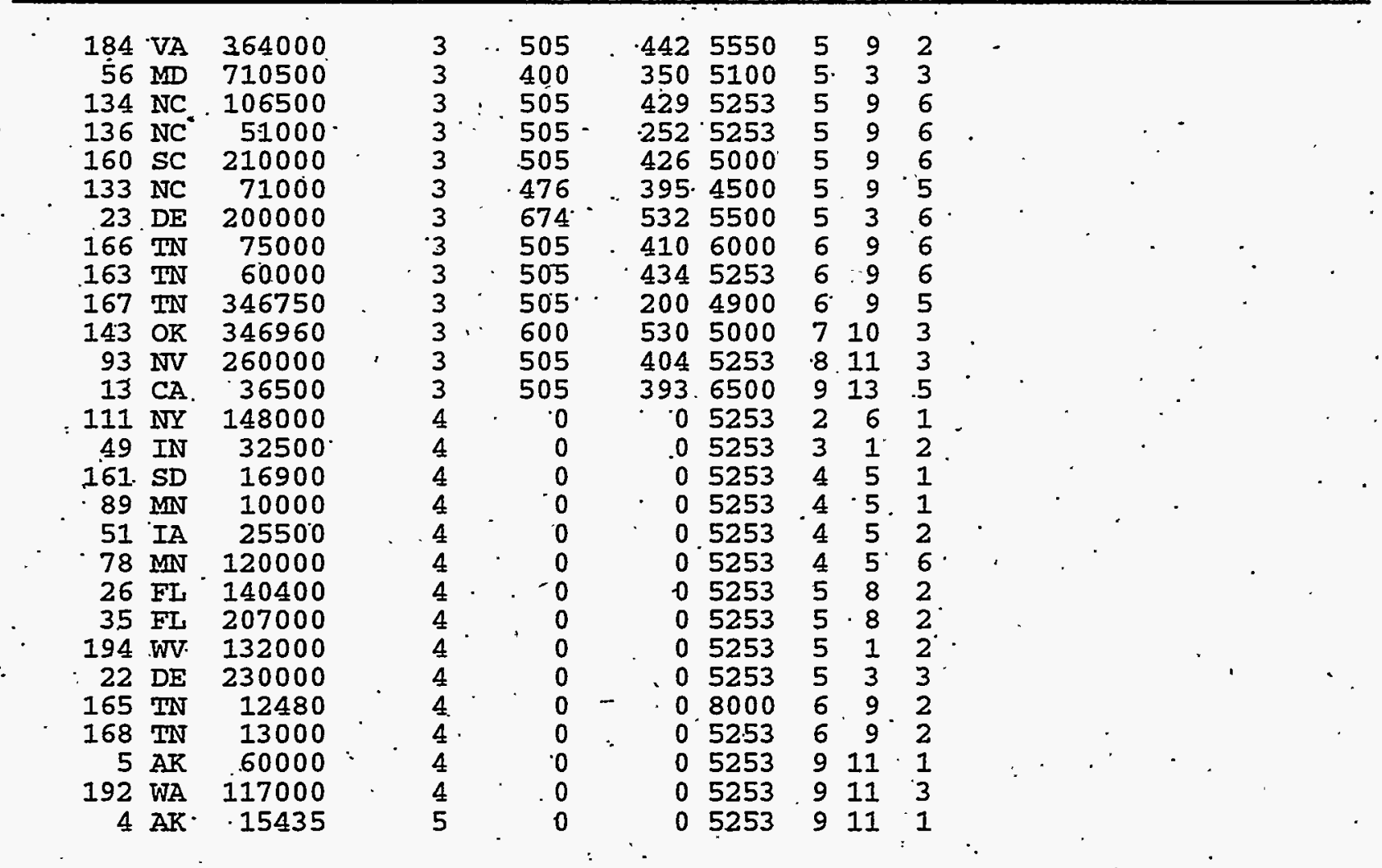

The FORTRAN program that determines $F, H R$, and MAPCRNR is listed below:

C MSWGD1.FOR * . CALCULATES F,MAPCRNR, MAPNRCR, HR, ERATIO

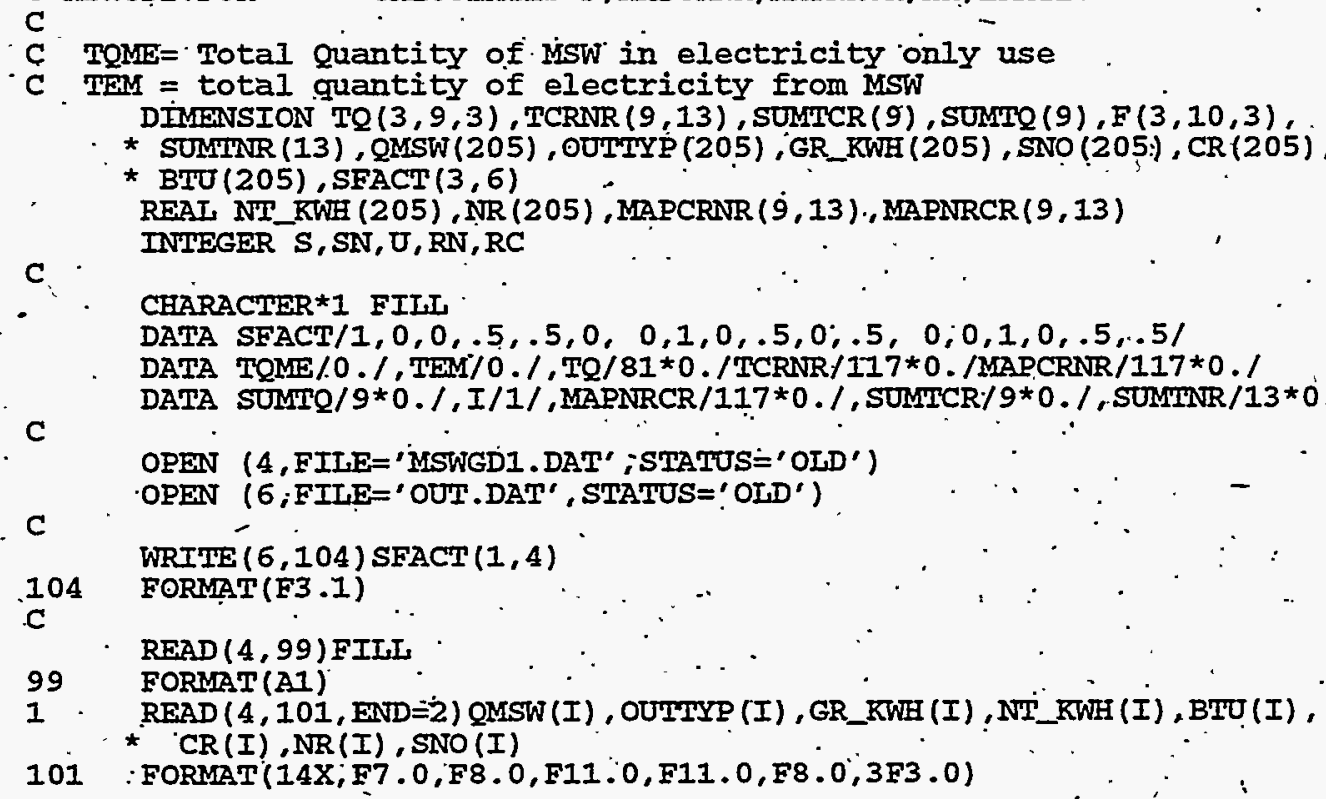




\section{The FORTRAN program that determines F, HR, and MAPCRNR (Continued)}

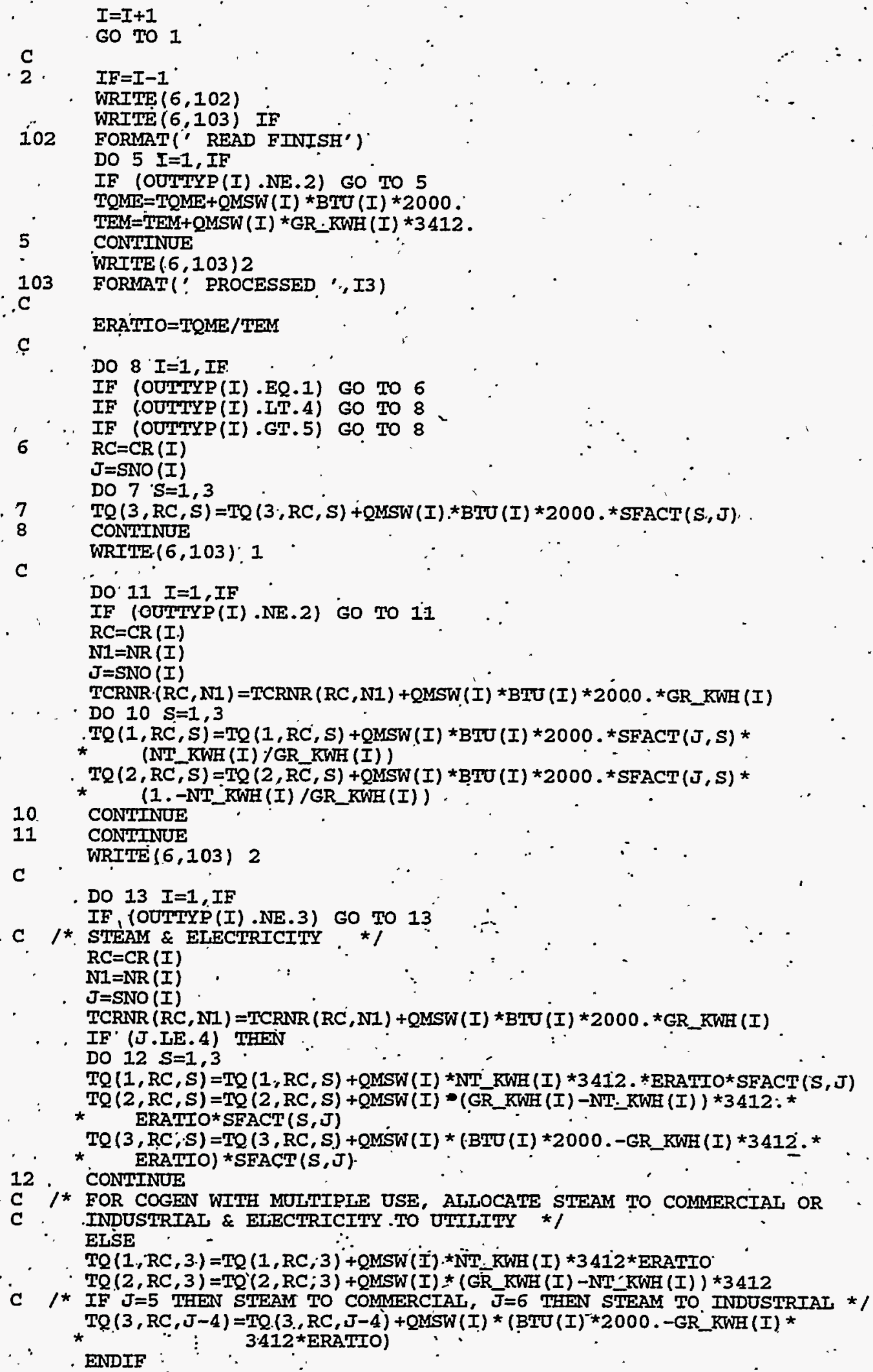




\section{The FORTRAN program that determines F, HR, and MAPCRNR (Continued)}

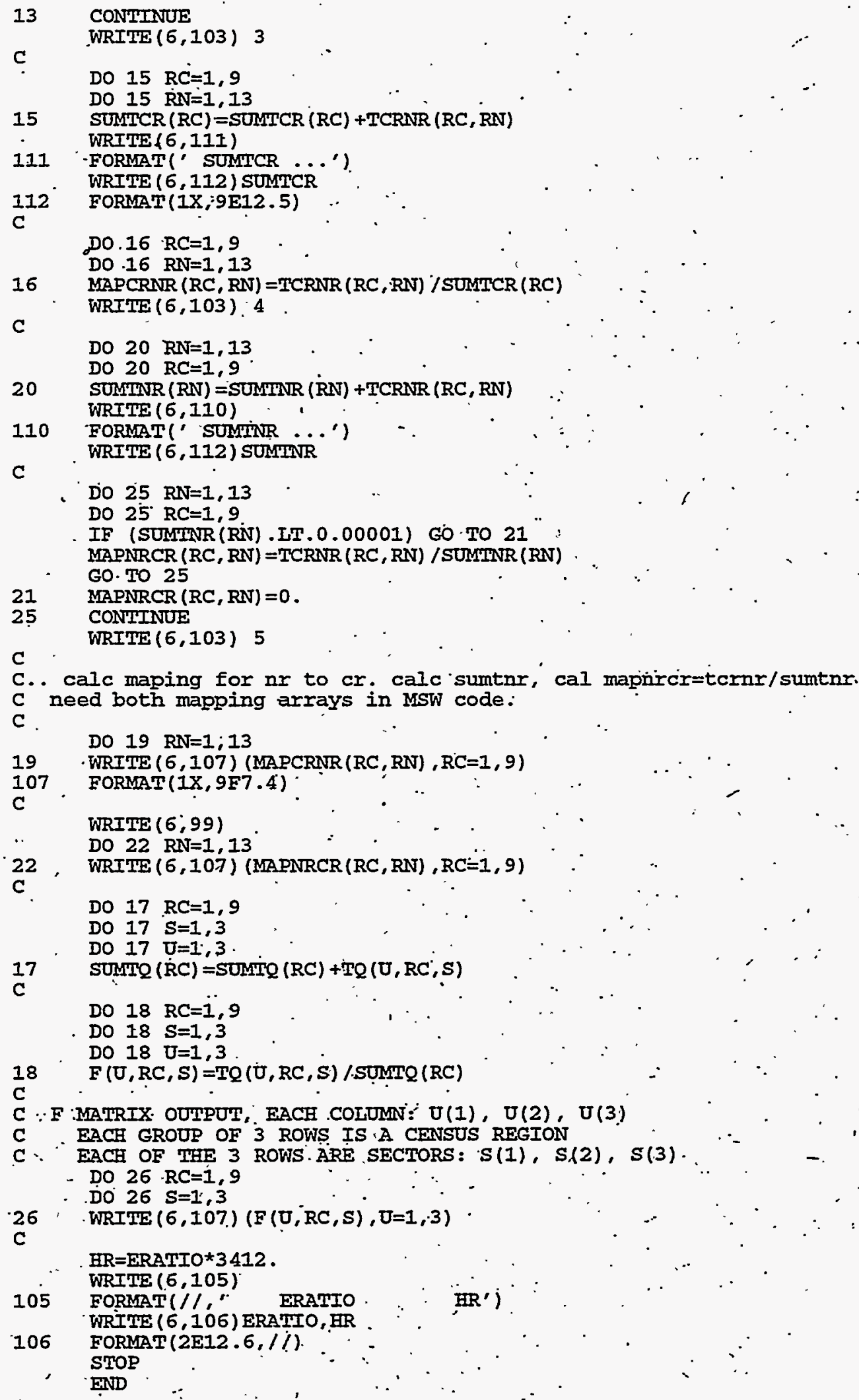




\section{Wind Energy Submodule (WES)}

\section{Model Purpose}

The objective of the Wind Energy Submodule (WES) is to project the cost, performance, and availability of wind-generated electricity, and provide this information to the Electricity Capacity Planning (ECP) component of the Electric Market Module (EMM) for the building of new capacity in competition with other sources of electricity generation.

Projections are based on the performance of a "mix" of horizontal axis wind turbines (HAWTs) that have been installed and are currently operational.

The version of NEMS used in AEO95. accounts for only grid-connected electricity generation. It does not consider dispersed, remote or any non-grid-connected applications. For wind, the grid connection or transmission costs are accounted for by the EMM, in the same manner as fossil generation technologies which may be less sensitive to siting, therefore understating these costs as pertains to wind.

The EMM provides to the WES information on installed wind capacity after convergence is reached. WES then calculates the remaining wind resources available for future installations. This accounting of remaining resources is needed since wind energy consists of limited quantities of high-quality resources that are depleted as turbines are installed on windy sites.

\section{Relationship of the Wind Submodule to Other Models}

As a submodule of the Renewable Fuels Module (RFM), WES provides its output through, and receives data through, the RFM. WES is initiated by a call from the RFM. The RFM then provides input to and receives data from the EMM.

The WES model calculates values for two variable arrays, which are then passed to the EMM for further processing. The calculated arrays are (1) yearly available capacity per wind class per region, and (2) yearly capacity factors for each wind class, region, and subperiod (i.e., "slice" of the load duration curve). The first array is calculated from the available land area versus wind class (average speed "bins"), the energy per unit swept rotor area; and the annual capacity factor. The second array is calculated from the subperiod energy percentages and subperiod definitions. - All other input data are passed directly to the EMM. The model generates a supply curve with a straightforward (deterministic) calculation from wind turbine performance projections.. The uncertainties in the results are related to the technological cost and performance projections and the assumptions about the availability of wind. 


\section{Modeling Rationale}

\section{Theoretical Approach}

The most important task of the WES is to produce energy supply curves from wind resource and wind turbine cost/performance data. This is accomplished by calculating, for three wind classes, the maximum conceivable turbine capacity that could be installed, given the available land area, wind resource, and the current year's turbine capacity factor.

Resource quality data and the yearly capacity factor are used to calculate wind farm performance data on a sub-yearly level, as required by the EMM. Calculations are made for each time slice, wind class, and region.

Substantial commercial wind installations have existed since the early 1980's. Counts of these pre-existing installations are used to adjust figures on available windy land at the beginning of. the NEMS model run. The WES tracks the quantity of windy land remaining by wind class that is available for future development after each run year by calculating the amount of resource required to provide a given amount of wind installed capacity and subtracting that amount from the total resource available. This assumes that the highest quality resource (as measured by average wind speed) is used first. These wind classes are represented by specific capacity factors for each region which correspond to time of day and season. The amount of resource, used is then subtracted from the previous year's available amount to yield the current year's available windy . land. A sample output for a given regional availability would be $50 \mathrm{MW}$ of Class 1 resource, $150 \mathrm{MW}$ of Class 2 , and $400 \mathrm{MW}$ of Class 3.

\section{Fundamental Assumptions}

\section{WES Quantity Projections}

The EMM requires capacity, performance, and cost data on the basis of NERC Regions. WES provides data by NERC Region based on 13 NERC Regions/Subregions with Alaska and Hawaii separated out; and not included in wind resources given to EMM.

Since horizontal-axis wind turbines are the predominant type in U.S. installations; accounting for over 95 percent of U.S. generating capacity, only this type is represented in the WES. No significant increase. in accuracy or detail would be achieved by including vertical-axis designs as well. The most current, comprehensive and accurate knowledge exists for the horizontal types and there are limitations on the detail with which projections of cost and performance can be made. For a regional model, the appropriate level of detail assumes a hybrid of various horizontal-axis turbines. 


\section{Land Use Estimates}

It is assumed that wind turbines are installed in a grid pattern with spacing between them equal to five times the rotor diameter in one direction and ten times the rotor diameter in the other direction.

\section{Dispersed Penetration}

It is assumed that penetration of dispersed wind energy systems will not impact the "learning curve" cost and performance changes of wind energy systems for central power generation. The two types of technologies' are different in scale and therefore the learning in one is not applicable to the other.

\section{Projected Btu Value of Wind Energy}

Energy balance computations and report writing and consumption rates within NEMS require a heat rate, i.e., an equivalent fossil-fuel displacement for wind generated electricity. This is currently set at the heat rate for fossil-fueled steam-electric plants of 10,302 .

\section{Alternative Approaches}

In most national-level energy models, wind technologies have not been considered on an equivalent basis with other sources of electricity generation. The few models that have are the Electric Power Research Institute's (EPRI) Electric Generation Expansion Analysis System (EGEAS) and the Environmental Defense Fund's (EDF) "Elfin". Also, DOE's Wind/Hydro/ Ocean Division has developed spreadsheet models that project utility market penetration of wind technologies based on comparisons of wind plant costs of energy (COE's) and marginal COE's for conventional generators.

EGEAS was developed jointly by EPRI and Stone and Webster. It consists of a set of computer programs for utility system planners which determines an optimal expansion plan or simulates a pre-specified plan. Expansion plans define the type, size, and installation date for each new generating facility. The objective is to find an expansion plan which minimizes the sum of operating expenses and capital fixed charges. ,EGEAS provides three main. optimization techniques which offer a balance between modeling flexibility and computational efficiency. EGEAS can handle a wide range of dispatchable and nondispatchable technologies, including wind.

The limitation of EGEAS with regard to renewables is that the variability or intermittency of wind resources is not explicitly incorporated into the model but rather is treated as a deterministic negative load, (e.g., as an hourly time series of power outputs over a year) and simply subtracted 
from utility demand. Therefore, wind is not explicitly competed or dispatched against other energy forms on an equal basis.

The ELFIN model from EDF, which stands for Electric Utility Financial and Production Cost Model, is a probabilistic model which simulates electric-system dispatch in order to calculate expected cost of operation. It has been used most extensively.in utility rate hearings before state energy commissions. Elfin can also be used to choose the optimal expansion plan for a utility based on annual present-value of system costs. and benefits. No attempt is made to compare lifecycle costs and benefits. Elfin's outputs include the generating level of each plant, per week, and year,fixed and variable costs, fuel usage, and emissions. Reliability is measured by loss-of-load probability (LOLP) and is displayed in days per year.

The DOE Wind/Hydro/Ocean Division's Model projects the growth of the U.S. electric utility market for wind turbines on a regional basis. Market share to the year 2030 is allocated on the basis of financial attractiveness, market acceptance of the technology, plant types and capacities, coincidence of utility load and wind power curves, wind resource limitations, and limitations on wind penetration into regional power pools.

The model is built around concepts of new product diffusion into the marketplace. It is a spreadsheet-based tool that estimates market capture in competition with conventional fossil fuelfired generating plants on a regional basis. It expands on previous techniques by incorporating a market acceptance factor based on ratios of levelized costs of energy for conventional plants and wind turbines (benefit cost ratios). Although sensitivities to fuel costs. and mixes can be evaluated with this model, nonfinancial, political factors cannot be incorporated so it is of limited usefulness for other purposes such as policy analyses.

\section{Wind Energy Submodule Structure}

\section{Submodule Flow Diagram}

A flow diagram showing the main computational steps and relationships of the Wind Energy Submodule is shown in Figure 2.

\section{Key: Computations and Equations}

Some of the input data are at 5-year intervals. The first calculation performs a linear interpolation on these data to calculate yearly values.

For the first year, subroutine CVINS is called to calculate the land area remaining for wind energy development, after deducting from the total windy land area available that needed to generate the existing installed capacity. This calculation is performed for each wind class, with capacity assigned first to wind class 1 land area, followed by wind class 2 land area when the 
Figure 2. Wind Energy Submodule Flowchart,

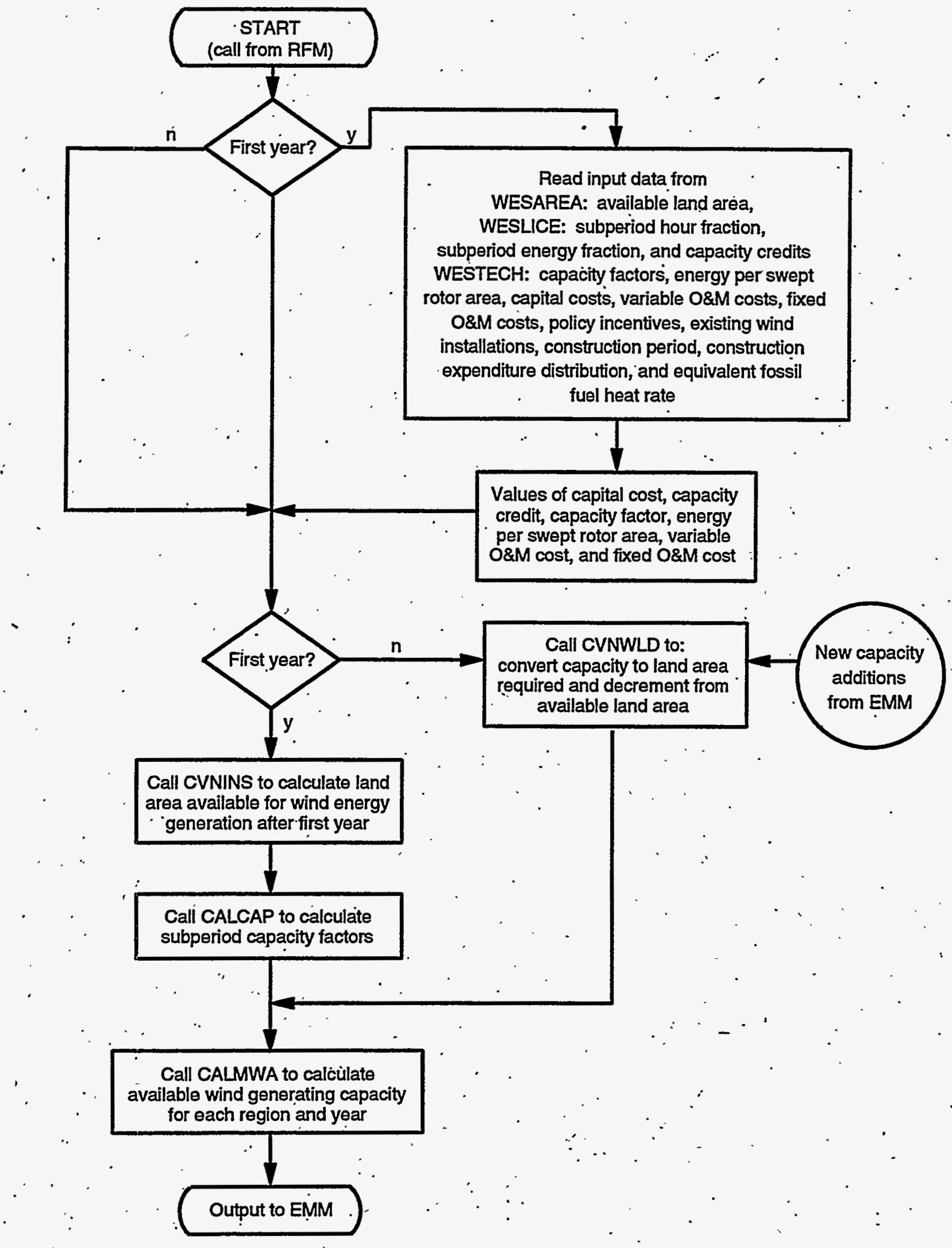


wind class 1 land area is completely used, and then similarly to wind class 3 land area. The calculation assumes a turbine spacing of $5 \mathrm{D} \times 10 \mathrm{D}$, where $\mathrm{D}$ is the diameter of the turbine rotor.

Following the calculation of land area used by pre-existing wind generating capacity, subroutine CALCAP is called to calculate the capacity factor for each region, year, and subperiod (time slice).

For all years after the first year, subroutine CVNWLD is then called to calculate the land area remaining for wind energy development; after deducting from the previously remaining land area available the land area needed for the amount of wind generating capacity installed in the previous year: This calculation is performed for each wind class, with capacity assigned first to wind class 1 land area, followed by wind class 2 land area when the wind class 1 land area is completely used, and then similarly to wind class 3 land area. The calculation assumes a turbine spacing of $5 \mathrm{D} \times 10 \mathrm{D}$, where $\mathrm{D}$ is the diameter of the turbine rotor.

Subroutine CALMWA is then called to convert the land area available for wind generation development to the swept rotor area needed to fully develop the available land area. The caiculation assumes a turbine spacing of $5 \mathrm{D} \times 10 \mathrm{D}$, where $\mathrm{D}$ is the diameter of the turbine rotor. This swept rotor area is then converted to the amount of wind energy generation capacity available in each region for each year and each.wind class. 


\section{Appendix 3-A: Inventory of Variables, Data, and Parameters}

This Appendix describes the variables, parameter estimates, and data inputs associated with the Wind Energy Submodule. Table 3A-1 provides a tabular listing of model variables and parameters.' The table contains columns with information on item definitions, modeling dimensions, data sources, measurement units, and documentation page references.

The remainder of Appendix 3-A consists of detailed descriptions of data injuts and variables, including discussions on supporting data assumptions and transformations.

Table 3A-1. NEMS Wind Energy Submodule Inputs and Outputs

\begin{tabular}{|c|c|c|c|c|}
\hline 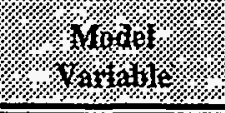 & 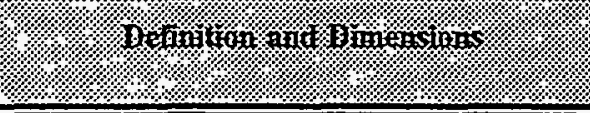 & ?. & (1) & Pepreference \\
\hline INPUT DATA & $=$ & & & \\
\hline CAPCOS & $\begin{array}{l}\text { Installed capital cost of wind generation in } \\
\text { NERC Region } n \text { in year } y \text {. }\end{array}$ & EPRI TAGMs, 1993. & $\$ \pi W$ & 74 \\
\hline CFANN & $\begin{array}{l}\text { Annual wind capacity factor for wind class } \\
w \text { in year } y \text {. }\end{array}$ & SAIC, 1990. & Unitless & 74 \\
\hline CREDIT & Wind capacity credit for NERC Region $n$ in & Determined within EMM. & Unitless & 75 \\
\hline & year $y$. & & & \\
\hline . ENAREA & $\begin{array}{l}\text { Energy per swept rotor area for wind class } \\
w \text { in year } y \text {. }\end{array}$ & SAIC, 1990. & $\mathrm{kWh} / \mathrm{m}^{2}$ & 75 \\
\hline EXWIND & Pre-existing total wind electric capacity & DOE Wind Program & MW & 76. \\
\hline HEATT & Fossil fuel equivalent heat rate for wind. & EIA, 1992. & Btu $/ \mathbf{k W h}$ & 76 \\
\hline$L E A D^{*}$ & Construction lead time. & EPRI TAGRM + 1 year. & Years & 76 \\
\hline OMFCOS & $\begin{array}{l}\text { Fixed O\&M cost for NERC Region } n \text { in } \\
\text { year } y \text {. }\end{array}$ & EPRI TAGM, 1993. & $\$ / k W$. & 77 \\
\hline OMVCOS & $\begin{array}{l}\text { Variable } O \& M \text { cost for NERC Region } n \text { in } \\
\text { year } y \text {. }\end{array}$ & EPRI TAGTM, 1993. & mills $/ \mathrm{kWh}$ & 77 \\
\hline PERCON & $\begin{array}{l}\text { Fraction of construction completed in each } \\
\text { year of construction. }\end{array}$ & EPRI TẠGM, 1993. & Unitless & 77 \\
\hline POUCY & $\begin{array}{l}\text { Policy incentives for NERC Region } n \text { in } \\
\text { year } y \text {. }\end{array}$ & Energy Policy Act of 1992. & mills $/ \mathbf{k W h}$ & 77 \\
\hline $\begin{array}{r}. \quad S L C E \\
-\end{array}$ & $\begin{array}{l}\text { Hour fraction for subperiod } l \text { in NERC } \\
\text { Region } n\end{array}$ & $\begin{array}{l}\text { WNDSLICE preprocessing } \\
\text { program (PERI). }\end{array}$ & Unitless & 78 \\
\hline
\end{tabular}


Table 3A-1. NEMS Wind Energy Submodule Inputs and Outputs (Continued)

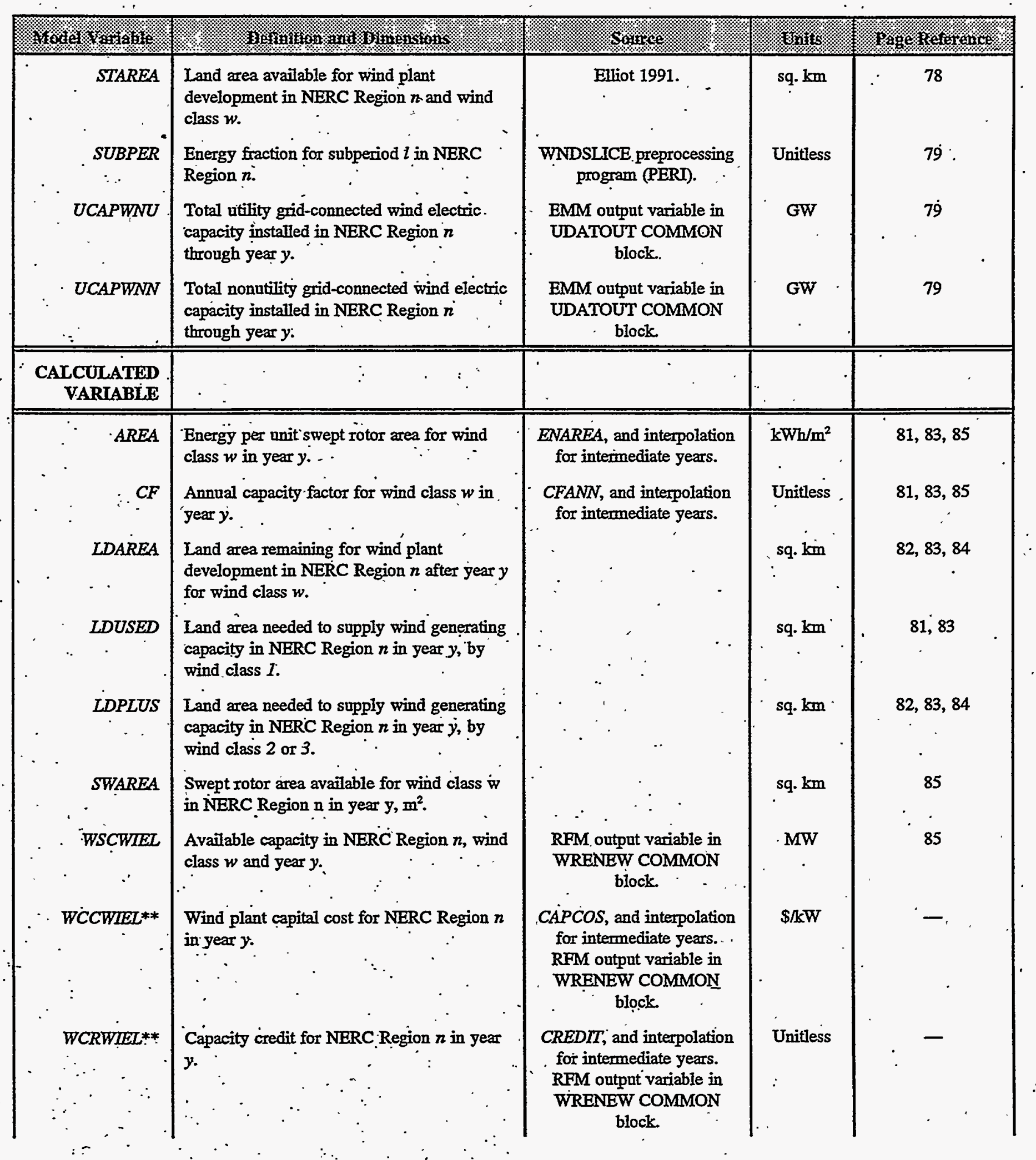


Table 3A-1. NEMS Wind Energy.Submodule Inputs and Outputs (Continued)

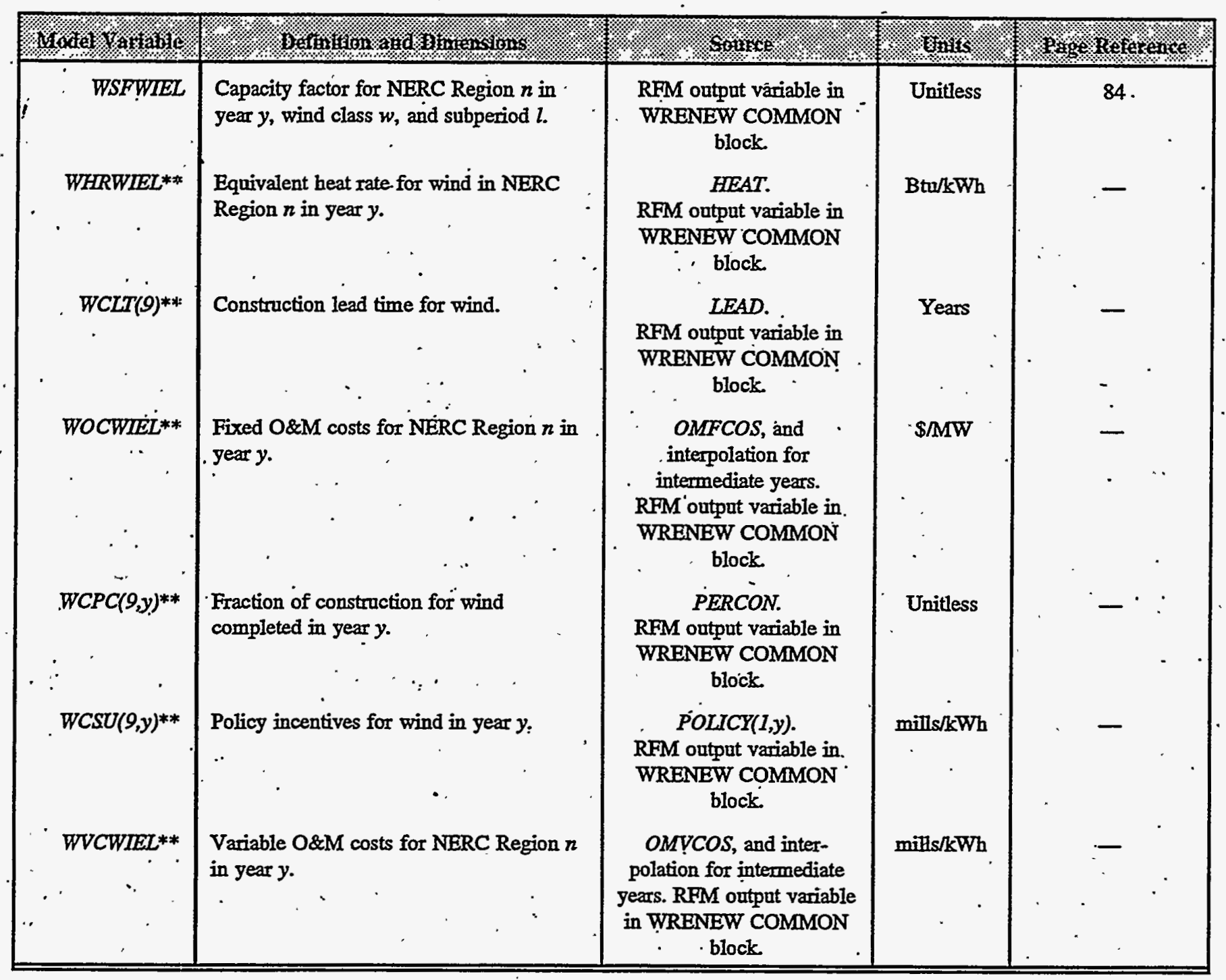

*Three years is minimum build time allowed for AEO95.

**Intermediate values, linearly interpolated from the source variable. 


\section{MODEL INPUT: · CAPCOS}

DEFINITION: : Installed capital cost of wind generation in NERC Region $n$ in year $y$ at 5year intervals; $(\$ / \mathrm{kW})$.

Values of capital cost are read into the WES from the WESTTECH data file. This value.is passed to EMIM and represents a capital cost after all learning has taken place exclusive of contingencies and is deflated to 1987 dollars. This value is $\$ 690$.

SOURCE: Electric Power Research Institute, TAGM - Technical Assessment Guide, 1993.

\section{MODEL INPUT: . CFANN}

\section{DEFINITION: $\quad$ Annual wind capacity factor for wind class $w$ in year $y$; (Unitless).}

Cuirrent performance estimates are based on a composite analysis of commercial turbines. Performance data are based on expert judgment projected for 5-year intervals. Specifically, 1995 data are based on the improvements expected from a turbine similar in technological development to the U.S. Windpower 33M-VS. For the years 2000 and 2005 , data are based on the improvements expected as a result of the DOE Wind Energy Program R\&D, with improvements. built up on a subsystem level: Beyond 2005 there is a higher degree of uncertainty regarding technology advances, so small incremental improvements are assumed.

Performance pfojections are taken from the accelerated federal wind technology R\&D funding scenario used in the 1990 National Energy Strategy technology characterizations. Because the characterizations termed "accelerated" in 1990 assumed a funding level for the Federal R\&D Program that closely duplicates the prevailing levels, and because of cooperative programs with industry and utilities, the 1990 accelerated scenario is representative of actual technology development.

SOURCES: Science Applications International Corporation, "Renewable Energy Technology Characterizations,". Report in.support of the National Energy Strategy for the U.S: Department of Energy, Office of Conservation and Renewable Energy, October 1990. :

Science Applications International Corporation, "Renewable Energy Technology Evolution Rationales," Report in support of the National Energy Strategy for the U.S. Department of Energy, Office of Conservation and Renewable Energy, October 1990. 


\section{MODEL INPUT: $\quad$ CREDIT}

DEFINITION: Wind capacity credit for NERC Region $n$ in year $y$ at 5-year intervals; (Unitless).

The Load Capacity Credit (LCC) or capacity value that can be attributed to intermittent generators is a debated issue. The percentage of rated power output for a wind generator that can be considered as firm capacity is dependent on the estimated change the generator effects in a specific utility system's loss-of-load probability (LOLP), generating mix, spinning reserve requirements, and other factors. Values of capacity credit are read into the WES from the WESTECH data file. This file currently assigns a value of zero to the capacity credit for all wind classes and all years. However, a value equal to three quarters of the capacity factor in the peak time period is assigned to the capacity factor in the Electric Capacity Planning Submodule of the EMM.

SOURCE: Value determined by EMM.

\section{MODEL INPUT: . ENAREA}

DEFINITION: $\quad$ Energy per swept rotor area for wind.class $w$ in year $y ;\left(\mathrm{kWh} / \mathrm{m}^{2}\right)$.

Current performance èstimates are based on a composite analysis of commercial turbines. Projected performance data are based on the "best knowledge" available at 5-year intervals. Specifically, 1995 data are based on the improvements expected from a turbine similar in technological development to the U.S. Windpower 33M-VS turbine. For the years 2000 and 2005, projections are based on the improvements expected as a result of the DOE Wind Energy Program R\&D, with improvements built up on a subsystem level. Beyond 2005 there is a higher degree of uncertainty regarding technology advances, and smaller incremental improvements are assumed.

Performance projections are taken from the accelerated federal wind technology R\&D funding scenario used in the 1990 National Energy Strategy technology characterizations. Because the characterizations termed "accelerated" in 1990 assumed a funding.level for the Federal R\&D Program that closely duplicates the prevailing levels, and because of cooperative programs with industry and utilities, the 1990 accelerated scenario is representative of actual technology development.

SOURCES: Science Applications International Corporation, "Renewäble Energy Technology Characterizations," Report in support of the National Energy Strategy for the U.S. Department of Energy, Office of Conservation and Renewable Energy, October 1990.

Science Applications International Corporation, "Renewable Energy Technology Evolution Rationales," Report in support of the National Energy Strategy for the 
U.S. Department of Energy, Office of Conservation and Renewable Energy, October 1990.

\section{MODEL INPUT: EXWIND.}

DEFINITION: Pre-existing total wind electric capacity installed in NERC Region $n$ through year $y ;(M W)$.

Substantial commercial wind installations have existed since the early 1980's. Counts of these pre-existing installations are stored in the input data file. These numbers are used to adjust figures on available windy land at the beginning of the NEMS model run.

SOURCE: DOE Wind Program records as maintained by Princeton Economic Research Inc. with data obtained from California Energy Commission, American Wind Energy Association, Pacific Gas and Electric, Southern California Edison, Sand Diego Gas and Electric, and other sources.

\section{MODEL INPUT: $\quad H E A T T$}

DEFINITION: - Fossil fuel equivalent heat rate for wind in NERC region $\mathrm{n}$ in year y in 5year intervals, (Btu/kWh).

This variable is not currently being used by the EMM An equivalent fossil fuel displacement value of 10,335 Btu/kWh has been assigned, based on EIA data for 1992.

SOURCE: Energy Information Administration, Annual Energy Review :1991, DOE/EIA-0384(91), June 1992.

\section{MODEL INPUT: LEAD}

DEFINITION: Construction lead time at 5 -year intervals; (Years).

The construction period for a wind generating station is currently set at 3 , which is the minimum lead time allowed in the EMM.

SOURCE: . Electric Power Research Institute, TÁGM - Technical Assessment Guide, 1993. 


\section{MODEL INPUT: OMFCOS}

DEFINITION: Fixed O\&M costs for NERC Region $n$ in year $y$ at 5-year intervals; $(\$ / \mathrm{kW})$.

Values of fixed O\&M costs are read into the WES from the WESTECH data file. Fixed O\&M costs are currently set in at $\$ 20.86 / \mathrm{kW}$ (1987 dollars) for.all years and all regions, based on the 1993 TAGRM.

SOURCE: . Electric Power Research Institute, TAGTM_Technical Assessment Guide, 1993.

\section{MODEL INPUT: OMVCOS}

DEFINITION: - Variable O\&M costs for NERC Region $n$ in year $y$ at 5-year intervals; (mills $/ \mathrm{kWh}$ ).

The variable O\&M costs are currently set at zero for all years and all regions based on the 1993 TAG TM.

SOURCE: : Electric Power Research Institute, TAGTM_Technical.Assessment Guide, 1993.

\section{MODEL INPUT: PERCON}

DEFINITION: Fraction of construction completed in each year of construction at 5-year intervals; (Unitless).

The construction period for a wind generating station is currently set at 3 years. The construction fraction is set at 0,10 percent, and 99 percent, respectively.

SOURCE: · Electric Power Research Institute, TAGTM-Technical Assessment Guide; 1993.

\section{MODEL INPUT: $\quad P O L I C Y$}

DEFINITION: - Policy incentives for NERC Region $n$ in year $y$ at 5-year intervals; (mills/kWh):

Any production incentives or other adjustments to the cost of wind energy are accounted for in the POLICY variable. Currently, a value of 15 mills.per kilowatt hour for the years 1994 through 2003 and zero for all other years is assigned for all regions. This is based on the policy incentive provision of the Energy Policy Act of 1992.

SOURCE: : Energy Policy Act of 1992 (Public Law 102-486), Section 1212. 


\section{"MODEL INPUT: SLICE}

DEFINITION: $\quad$ Hour fraction for subperiod $l$ in NERC Region $\dot{n}$; (Unitless).

Data for 20 subperiods of the year are provided. The EMM maps the data for these 20 subperiods into nine subperiods used in the EMM and other NEMS modules.

SOURCE: Princeton Economic Research Incorporated (PERI), WNDSLICE preprocessor program, Bertrand L. Johnson.

\section{MODEL INP.UT: $\quad$ STAREA}

DEFINITION: . Land area available for wind plant development in NERC Region $n$ and wind class $w$; (sq. $\mathrm{km})$.

The land area available for wind plant development has been extracted from data produced at Battelle Pacific Northwest Laboratory (PNL) in support of DOE's National Energy Strategy. In producing the Wind Energy Resource Atlas, PNL staff attempted to account for variations in such . factors as anemometer height and placement through measures such as making determinations regarding the validity of data and extrapolating the wind speeds to a standard height.

'PNL developed their areal assessments of available-resource by breaking down their wind resource maps into one-third degree longitude by one-quarter degree latitude grids. These grid cells formed the basic unit for which wind power and land availability were estimated. Because of resolution limitations, details of wind resource were lost, particularly in mountainous and coastal areas. Since wind speed estimates in mountainous regions apply only to those areas free of obstructions, only a fraction of the ares shown in the Atlas are actually available for development. These fractions were estimated by PNL when producing areal estimates.

PNL developed scenarios covering a.range of.land exclusion amounts. The WES input data are based on the "moderate" exclusion scenario, which excludes all environmentally.protected lands (such as parks and wilderness areas), all urban lands; all wetlands, 50 percent of forest lands, 30 percent of agricultural lands, and 10 percent of range and barren lands. Only land areas with áverage wind speeds above $12.5 \mathrm{mph}$ are included in the WES input data. Land areas : are separated into three classes, depending on the range of average wind speeds. WES class 1 is for average wind speeds above $14.3 \mathrm{mph}$, class 2 is for-average wind speeds from $13.4 \mathrm{mph}$ to 14.3 $\mathrm{mph}$, and class 3 is for average wind speeds from $12.5 \mathrm{mph}$ to $13.4 \mathrm{mph}$, all at a height of 10 meters. 
SOURCES: Elliott, D.L., et al, "An.Assessment of the Available Windy Land Area and Wind Energy Potential in the Contiguous United States," Pacific Northwest Laboratory; Report \#PNL-7789, August 1991.

Elliott, D.L., et al, "Wind Enérgy Resource Atlas" (12 volumes), Pacific Northwest Laboratory, Report PNL-3195; 1980.

\section{MODEL INPUT: $\quad$ SUBPER}

DEFINITION: : Energy fraction for subperiod $l$ in NERC Region $n$; (Unitless).

Values were calculated using WNDSLICE, a preprocessing program developed by Princeton Economic Research Incorporated. WNDSLICE uses established NEMS subperiod definitions; daily and seasonal wind resource data, and a synthetic wind turbine power curve to estimate the fraction of the annual wind energy production that falls within the various subperiods.

SOURCE: Princeton Economic Research Incorporated, WNDSLICE preprocessor program:

\section{MODEL INPUT: $\quad U C A P W N N$}

DEFINITION: - Total nonutility grid-connected wind electric capacity installed in NERC Region $n$ through year $y$; (GW).

SOURCE: EMM output variable in UDATOUT COMMON block.

\section{MODEL INPUT: UCAPWNU}

DEFINITION: Total utility grid-connected wind electric capacity installed in NERC Region $n$ through yéar $y_{;}$(GW).

SOURCE: EMM output variable in UDATOUT COMMON block. 



\section{Appendix 3-B: Mathematical Description}

This Appendix provides the detailed mathematical specification of the Wind Energy Submodule as presented in the RFM FORTRAN code execution sequence. Subscript definitions are also as they appear in the FORTRAN code.

Subroutine CVNINS (used only for first year)

Equation 3B-1 calculates the land area (in. $\mathrm{sq} . \mathrm{km}$ ) needed to supply a particular amount of wind generating capacity in a particular NERC Region, year and wind class:

$$
\operatorname{LDUSED}_{n, 1, w}=\frac{\operatorname{SXWIND}_{n, 1} \times C F_{1, w} \times 8760 \times \alpha^{S P}}{A R E A_{1, w} \times \frac{\pi}{4}} \times C_{\mathrm{conv}}
$$

where:

LDUSED $_{m, 1, w} \quad=\quad$ Land area needed to supply pre-existing wind generating capacity in NERC Region $n$ in year 1 , by wind class $w$, sq. $\mathrm{km}$,

EXWIND $D_{m} \quad \cdot=\quad$ Pre-existing total wind electric capacity installed in NERC Region $n$ through year $1, \mathrm{MW}$,

$C F_{1, w} \quad=\quad$ Annual capacity factor for wind class $w$ in year 1,

$A R E A_{1, w}$. = Energy per unit swept area for wind class $w$ in year $1, \mathrm{kWh} / \mathrm{m}^{2}$,

$\pi \quad=\quad$ = 3.141593 .

$\alpha^{S p}=\quad$ Scalar derived from $5 \mathrm{D} \times 10 \mathrm{D}$ grid spacing of wind generator $\left(\alpha^{S P}=50\right)$,

$\begin{gathered}=\quad \text { Conversion factor } \\ \therefore\end{gathered}\left(\frac{M W \times h}{\frac{k W h}{m^{2}}}\right)=10^{-3}\left(\mathrm{~km}^{2}\right)$ 
Equations 3B-2 and 3B-3 calculate the remaining windy land area available for development for wind classs w:

If $L D U S E D_{n, 1, \dot{w}}<S T A R E A_{n, w}$, then

$$
\begin{aligned}
\text { LDAREA }_{n, 1, w} & =\text { STAREA }_{n, w}-\text { LDUSED }_{n, 1, w} \\
\text { LDPLUS }_{n, 1, w+1} & =0.0
\end{aligned}
$$

If $L D U S E D_{n, 1, w} \geq S T A R E A_{n, w}$, then

$$
\begin{aligned}
\operatorname{LDAREA}_{n, 1, w} & =0.0 \\
\operatorname{LDPLUS}_{n, 1,2} & =\left[\frac{A R E A_{1,1}}{A R E A_{1,2}}\right] \times\left[\frac{C F_{1,2}}{C F_{1,1}}\right] \times\left(L D U S E D_{m, 1,1}-S T A R E A_{n, 1}\right)
\end{aligned}
$$

where:

$$
\begin{array}{ll}
\text { LDAREA }_{n, 1, w}= & \begin{array}{l}
\text { Wind class } w \text { land area available for development in NERC Region } \\
n \text { after year } 1, \mathrm{sq} \cdot \mathrm{km},
\end{array} \\
S T A R E A_{n, w} & =\quad \begin{array}{l}
\text { Total wind class, } w \text { land area available for development in NERC } \\
\text { Region } n, \text { sq. } \mathrm{km},
\end{array} \\
L D P L U S_{n, 1, w}= & \begin{array}{l}
\text { Land area needed to supply pre-existing wind generating capacity } \\
\text { in NERC Region } n \text { in year } 1, \text { by wind class } w, \text { sq } \mathrm{k} .
\end{array}
\end{array}
$$

Equations 3B-4 and 3B-5 compute the amount of windy land area available for development at the beginning of a particular year by NERC Region and wind class:

If $L D P L U S_{n, 1,2}<S T A R E A_{n, 2}$, then

$$
\begin{aligned}
& \text { LDAREA }_{n, 1,2}=S T A R E A_{n, 2}-\text { LDPLUS }_{n, 1,2} \\
& \text { LDAREA }_{n, 1,3}=S T A R E A_{n, 3}
\end{aligned}
$$


If $L D P L U S_{n_{1,2}} \geq S T A R E A_{n, 2}$, then

$$
\begin{aligned}
\operatorname{LDAREA}_{n, 1,2} & =0.0 \\
\operatorname{LDPLUS}_{n, 1,3} & =\left[\frac{A R E A_{1,2}}{A R E A_{1,3}}\right] x\left[\frac{C F_{1,3}}{C F_{1,2}}\right] x\left(L D P L U S_{n, 1,2}-S T A R E A_{n, 2}\right) \\
\operatorname{LDAREA}_{n, 1,3} & =S T A R E A_{n, 3}-L D P L U S_{n, 1,3}
\end{aligned}
$$

\section{Subroutine CVNWLD (not used for first year)}

Equation 3B-6 calculates the land are (in sq. $\mathrm{km}$ ) needed to supply the wind generating capacity called for by the EMM by NERC Region, year and wind class:

$$
\text { LDUSED }_{n, y, 1}=\frac{\left(U C A P W N U_{n y}+U C A P W N N_{n y}-U C A P W N U_{n y-1}-U C A P W N N_{n y-1}\right) \times C F_{y, 1} \times 8760 \times \alpha^{80}}{: A R E A}
$$

where:

$$
\begin{aligned}
& . \text { LDUSED }_{n, y, w} \quad=\quad \text { Land area used to supply EMM-called for wind generating capacity. } \\
& \text { in NERC Region } n \text { in year } y \text {, by wind class } w \text {, sq. } \mathrm{km} \text {, } \\
& U C A P W N U_{n y}=\text { Total utility grid-connected wind electric capacity installed in } \\
& \text { NERC Region } n \text { through year } y, \mathrm{GW} \text {, } \\
& U C A P W N N_{m, y}=\cdots \text {. Total nonutility grid-connected wind electric capacity installed in . } \\
& \text { NERC Region } n \text { through year } y \text {, GW, } \\
& C F_{y, w} \quad=\quad \text { Annual capacity factor for wind class } w \text { in year } y \text {, } \\
& A R E A_{y, w} \quad=\quad \begin{array}{l}
\text { Energy per unit swept rotor area for wind class } w \text { in } y e a r y, \\
\mathrm{kwh} / \mathrm{m}^{2}
\end{array} \\
& \pi \quad=3.141593 \text {, } \\
& \alpha^{5 P} \quad=\quad \text { Scalar derived from 5D } \times 10 \mathrm{D} \text { grid spacing of wind generator } \\
& \left(\alpha^{s}=50\right) \text {. }
\end{aligned}
$$

Equations 3B-7 calculates the land area needed to supply the wind generating capacity called for by the EMM when there is sufficient remaining land area to fulfill the request. 
If $L D U S E D_{m, 3, w}<L D A R E A_{m, y-1, w}$, then

$$
\begin{aligned}
\text { LDAREA }_{n y, w} & =\text { LDAREA }_{n, y-1, w}-L D U S E D_{n_{y, w}} \\
\operatorname{LDPLUS}_{n y, w+1} & =0.0
\end{aligned}
$$

Equation 3B $\leq 8$ calculates the land area needed to supply the wind generating capacity called for by the EMM when the next lower quality wind resource has to be used because of insufficient remaining land of a higher quality. Since lower quality wind classes have lower capacity factors, the capacity factors serve as multipliers to adjust the amount of wind land area needed.

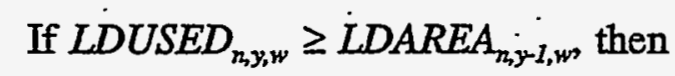

$$
\begin{aligned}
& L^{L D A R E A_{n y, w}}=0.0 \\
& \operatorname{LDPLUS}_{n, y, w+1}=\left[\frac{A R E A_{y, w}}{A R E A_{y, w+1}}\right] \times\left[\frac{C F_{y, \dot{w}+1}}{C F_{y, w}}\right] x\left(L D U S E D_{n, y, w}-L D A R E A_{n, y-1, w}\right) \\
& \operatorname{LDAREA}_{n, y, w+1}=L D A R E A_{n, y-1, w+1}-L D P L U S_{n, y, w+1} \quad \text { for } w=1,2,3
\end{aligned}
$$

where:

$$
\begin{aligned}
& L_{L D A R A}=\quad \text { Wind class } w \text { land area available for development in NERC Region } \\
& n \text { after year } y \text {, sq. } \mathrm{km} \text {, } \\
& \text { LDPLUS }_{m, w} \quad \text { = Land area needed to supply EMM-called for wind generating } \\
& \text { capacity in NERC Region } n \text { in year } y \text {, by wind class } w \text {, sq. } \mathrm{km} \text {. }
\end{aligned}
$$

\section{$\underline{\text { Subroutine CALCAP }}$}

Equation 3B-9 calculates the capacity factor for a particular wind class, NERC Region, year and subperiod:

$$
\begin{aligned}
& \text { WSFWIEL }_{n, y, w, l}=\left[\frac{\text { SUBPER }_{n, l}}{\operatorname{SLICE}_{n, l}}\right] \times \dot{C F}_{y, w} \\
& \text { WSFWIEL } L_{i n, y, 1, l}=W S F W E L_{n, y, w_{n} l}
\end{aligned}
$$


"where:

WSFWIEL $_{n, y, l}=\quad \begin{aligned} & \text { Capacity factor for wind class } w \text { in NERC Region } n \text { in year } y \text { in } \\ & \text { subperiod } l\end{aligned}$
$S U B P E R_{n, l} \quad=\quad \begin{aligned} & \text { Energy fraction for subperiod } l \text { in NERC Region } n \\ & S L I C E_{n, l}\end{aligned} \quad=\quad$ Hour fraction for subperiod $l$ in NERC Region $n$,
$C F_{y, w} \quad=\quad$ Annual capacity factor for wind class $w$ in year $y$,

Subroutine CALMWA

Equation 3B-10 computes the total swept area by turbines for a particular wind class, NERC Region and year:

$$
S W A R E A_{n_{y, w}, w}=\frac{\frac{\pi}{4} \times L D A R E A_{n_{y, w}} \times 10^{6}}{\alpha^{S p}}
$$

where:

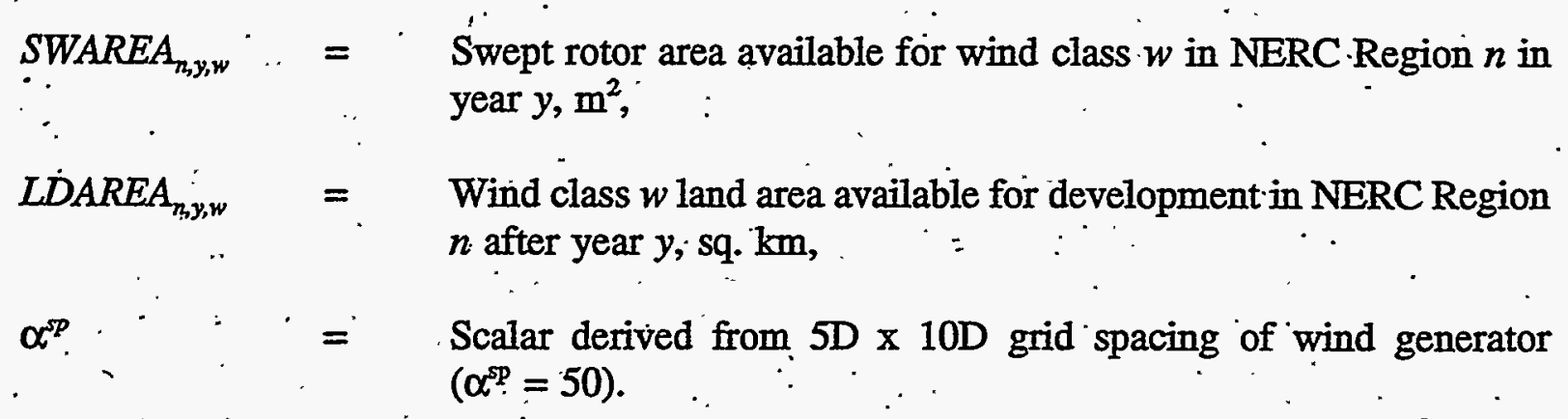
$L \dot{D} A R E A_{r, y, w} \quad=\quad$ Wind class $w$ land area available for development in NERC Region $n$ after year $y$; sq. $\mathrm{km}$,

Equation 3B-11 computes the available wind electric generation capacity in megawatts by wind class, NERC Region and year:

$$
\text { WSCWIEL }_{n, y, w}=\frac{\text { AREA }_{y, w} \times S W A R E A_{n, y, w}}{C F_{y, w} \times 10^{3} \times 8760}
$$

where:

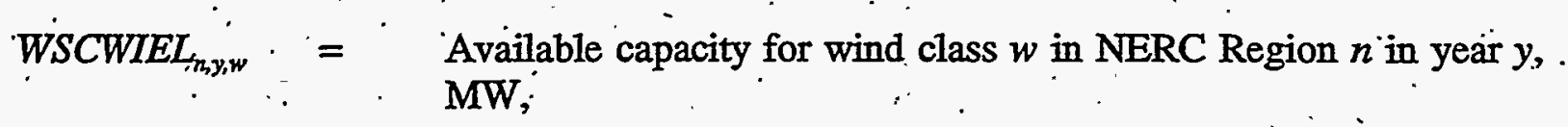


$A R E A_{y, w} \quad=\quad$ Energy. per unit swept rotor area for wind class $w$ in year $y$, $\mathrm{kWh} / \mathrm{m}^{2}$,

$C F_{y, w} . \quad=\quad$ Annual capacity factor for wind class $\ddot{w}$ in year $y$.

Equation 3B-12 computes the summation of the available wind generating capacity for all wind classes by NERC Region and year:

$$
\text { WCAWIEL } L_{n, y}=\sum_{w=1}^{3} \text { WSCWIEL } L_{n, y, w}
$$

where:

WCAẂIEL $L_{n,}=$ Available capacity for all wind classes through class 3 in NERC Region $n$ in year $y, M W$. 


\section{Appendix 3-C: Bibliography}

Cohen, J.M., Schweizer, T.C., Hock, S.M., and Cadogan, J.B., "A Methodology for Computing Wind Turbine Cost of.Electricity Using Utility Economic Assumptions;". Proceedings of Windpower '89, American Wind Energy Association, September 1989.

Elliott, D.L., et al; "An Assessment of the Available Windy Land Area and Wind Energy Potential in the Contiguous United States," Pacific Northwest Laboratory, Report PNL-7789, August 1991.

Elliott, D.L., et al., "Wind Energy Resource Átlas of the United States," (1 volume), Report DOE/CH 10093-4, October 1986.

Elliott, D.L., et al., "Wind Energy Resource Atlas," (12 volumes), Pacific Northwest Laboratory, Report PNL-3195, 1980.

Electric Power Research Institute, Technical Assessment Guide (TAG ${ }^{\mathrm{TM}}$ ), 1993.

Energy Information Administration, Annual Energy Review 1991, DOE/EIA-0384(91), 1992.

Energy Information Administration, "Component Design Report for Solar.and Wind Submodules Renewable Fuels Module National Energy Modeling System," Draft, April 23, 1993.

Garver, L.L., "Effective Load Carrying Capability of Generating Units," IEEE Transactions on Power Apparatus and Systems, August 1966.

Hock, S.M., Thresher, R.W., and Cohen, J.M.; "Performance and Cost Projections for Advanced. Wind Turbines," Proceedings of ASME Winter Annual Meeting, Dallas, TX, November 1990.

Princeton Economic Research, Incorporated, Data Development Report for Wind Electric Submodule Renewable Fuels Module National Energy Modeling System, February 24, 1993.

Princeton Economic Research, Incorporated, Software Overview Report for Wind Electric Submodule Renewable Fuels Module National Energy Modeling System, March 29, 1993.

Science Applications International . Corporation, "Renewable Energy Technology . Characterizations," Report in support of the National Energy Strategy for the U.S. Department of Energy, Office of Conservation and Renewable Energy, October 1990.

Science Applications International Corporation, "Renewable Energy Technology Evolution Rationales," Report in support of the National Energy Strategy for the U.S. Department of Energy, Office of Conservation and Renewable Energy, October 1990. 
Schweizer, T. and Cohen, J., "A Technique for Estimating Project Risk: Beyond Traditional Sensitivity Analyses," Science Applications International Corporation, Proceedings of the Windpower '88 Conference, September 18-22, 1988. 


\section{Appendix 3-D: Model Abstract}

Model Name:

Wind Energy Submodule

Model Acronym:

WES

\section{Description:}

Resource quality data and the yearly capacity factor are used to calculate wind farm performance data on a sub-yearly level, as required by the EMM. Calculations are made for each time slice, wind class, and region.

\section{Purpose of the Model:}

The purpose of the Wind Energy Submodule (WES) is to project the cost, performance, and availability of wind-generated electricity, and provide this information to the Electricity Capacity Planning (ECP) component of the Electric Market Module (EMM) for building the new capacity in competition with other sources of electricity generation.

\section{Most Recent Model Ujpdate:}

November 3, 1994

\section{Part of Another Model?:}

The Wind Energy Submodule is a component of the Renewable Fuels. Module (RFM) of the National Energy Modeling System (NEMS).

\section{Official Model Representative:}

Perry.M. Lindstrom

Coal, Uranium; and Renewable Fuels Analysis Branch

Energy Information Administration

(202) 586-0934

\section{Documentation:}

NEMS Documentation Report: Renewable Fuels Submodule, May 1995

\section{Archive Media and Installation Manual(s):}

Archived as part of the NEMS production runs.

\section{Energy System Described:}

A hybrid of various existing and proposed horizontal-axis wind turbines. Horizontal-axis wind turbines represent over 95 percent of U.S. generating capacity. 


\section{Coverage:}

- Geographic: 15 NERC Regions: East Central, Texas, Mid-Atlantic, Mid-America, MidContinent, Northeast, New England, Florida, Southeastern, Southwest, Western, Rocky Mountain, California and South Nevada, Aläska, and Hawaii -

- Time/Unit Frequency: Annual, 1990 through 2010

- Products: Electricity

- Economic Sectors: Electric utility sector, nonutility generators (NUGS)

- Model Structure: Sequential calculation of available wind capacity by NERC Region, wind class and year with a deduction of that year's installed capacity from the remaining available capacity

- Modeling Techniques: Accounting function of available windy land area and conversion of land area to swept rotor area and then to available generation capacity

- 'Special Features: Accounting for policy and/or production incentives.

\section{Modeling Features:}

\section{DOE Input Sources:}

Energy Information Administration, Annual Energy Review 1991, DOE/EIA-0384(91), June 1992.

Pacific Northwest Laboratory, Reports PNL-7789, DOE/CH 10093-4, and PNL-3195.

\section{Non-DOE Input Sources:}

Princeton Economic Research, Incorporated (PERD - WNDSLICE preprocessing program.

Science Applications International Corporation (SAIC) - Cost and peiformance data as prepared for the National. Energy Strategy project.

Electric Power Research Inștitute, Technical Assessment Guide (TAGTM), 1993.

\section{Computing Environment:}

- Hardware Used: IBM 3090

- Operating System: MVS :

- Language/Software Used: VS FORTRAN, Ver. 2.05

- Memory Requirement: $35 \mathrm{~Kb}$

- Storage Requirement: $23 \mathrm{~Kb}$ 
- Estimated Run Time: .06 seconds:

- Special Features: None.

\section{Independent Expert Reviews Conducted:}

None.

\section{Status of Evaluation Efforts by Sponsor:}

None. 



\section{Appendix 3-E: Data Quality and Estimation Processes}

This Appendix discusses (1) the quality of the principal sources of input data used in the Wind Energy Submodule, along with a discussion of user-defined parameters and guidelines used to select them, and (2) estimation.methods used to derive parameters.

Wind resources of the United States have been extensively charted and classified by the Pacific Northwest Laboratory (PNL). Three classes of wind resources, based on average annual wind speeds, are generally used. These classes correspond to PNL class 4 winds and higher, (speeds. greater than $5.6 \mathrm{~m} / \mathrm{s}(12.5 \mathrm{mph})$ ) which represent the generally-accepted, lowest economic limit of wind speeds for grid-connected systems in the United States.

Data on wind resource quantity are maintained in the Wind Resource Quantity File as derived from published assessments or compilations of U.S. wind resources. It contains regional data on the land area (in square kilometers) estimated to be available for wind plant development, accounting for the exclusion of some land as a result of environmental and land-use considerations.' WES uses the PNL "moderate" exclusion scenario. The percent of total windy land unavailable under this scenario consists of all environmentally protected lands (such as parks and wilderness areas), all urban lands, all wetlands, 50 percent of forest lands, 30 percent of agricultural lands, and 10 percent of range and barren lands. Within each region, the available land area is provided for each of the three levels of wind resource, according to the estimated average annual wind speed in that region and other factors. Lastly, since wind power increases significantly with height, a minimum height is usually specified for measurement and installation purposes, to achieve an associated wind power density.

The Wind Resource Quality File describes the variations in wind resource on a daily and seasonal basis, and estimates wind output during the different load condition subperiods to analyze the correlation with load profiles. The file is highly dependent on the raw wind speed file components chosen and incorporates data for many of the 975 stations in the Wind Energy Resource Information System (WERIS) from the National Climatic Data Center. The file also contains information on Load Duration Curve (LDC) subperiod definitions outside of the WES and the subperiod energy percentages. From this, WES estimates a capacity factor for a given subperiod. The specific subperiods correspond to season and time of day.

The Cost and Performance of Installed Wind Turbines have been monitored for almost a decade. During that period, a wind turbine database and turbine simulation program have been developed and refined. Also, analyses of manufacturer-supplied wind turbine power curves and installed costs were performed for a number of the best current, commercially available wind turbines. Wind turbine energy output estimates were made, assuming a Weibull wind speed distribution at several wind speeds, as well as corrections to wind speed for turbine hub height. Energy losses were based on field estimates from California wind plants. Average performance was estimated from the range of energy output data. Average costs were similarly calculated, and included

major repairs such as rotor replacements and O\&M costs. 
The Wind Turbine Cost and Performance Projections to be used initially for the WES data files are based on the accelerated Federal wind technology R\&D funding scenario used in the 1990 NES technology characterizations. The funding levels termed "accelerated" correspond-most closely to present levels and emphases, namely R\&D in the basic sciences and the "Advanced Wind Turbine" development program. There are also comprehensive cooperative programs with industry and utilities to assist in both near-term problem solving and long-term development.

The accelerated R\&D scenario assumes that a significant portion of the "advanced turbine" technology is available by 1995 , although scale-up to the complete $500 \mathrm{~kW}$ advanced turbine design is shown in year 2000. There is higher probability of success for the advanced turbine technology under the accelerated scenario because multiple designs. will be able to be tested, resulting in lowered technical risk and multiple learning curves to prevent the problem of technological "lock-out" as discussed in the Technology Penetration CDR. In addition, technology transfer and design assistance programs could speed adoption and improve quality of the new technology. Further incremental improvements are experienced by the year 2005, and scale up to an optimum ( $1 \mathrm{MW})$ turbine, utilizing significantly better design tools developed by the basic science element of the program, should occur in the 2010 time frame. Small, incremental improvements should follow after 2010.

Estimates for the mid-term technology characterizations were based on (1) projections for the U.S Windpower 33M-VS turbine, and (2) analysis conducted by NREL of potential advanced design improvements based on technical insights from the current $R \& D$ program. The general approach used in the-NREL analysis to determine the effects of design improvements on existing wind turbine technology can be described by three basic steps. First, a reference system was selected to represent current technology and its performance and costs were tabulated. Second, two configurations representing possible improvements to the reference design were identified, and the effect of each improvement on performance and cost was estimated. Lastly, estimated changes to wind plant cost of energy (COE) were calculated from the reference and improved design parameters. 


\section{Solar Submodule}

\section{Model Purpose}

The objective of the NEMS Solar Electric Submodule (SOLES) is to project the costs and performance characteristics of grid-connected Solar Thermal (ST) and Photovoltaic (PV) electricity generating technologies, and provide them to the Electricity Market Module (EMM) for dispatching these technologies in competition with other sources of electricity generation for the purpose of capacity expansion. Projections of costs and performance characteristics (including resource availabilities) are provided by EMM region and year. EMM Regions are based on the North American Electric Reliability Council (NERC) Regions, as modified by the Energy Information Administration (EIA) for NEMS. For PV technologies, all EMM Regions are represented in SOLES. For ST technologies, however, only six selected regions are represented, since this technology will not compete in all regions of the country. The six selected regions include essentially all land west of the Mississippi River. The available solar resource is well in excess of conceivable demand (2.orders of magnitude or more). Therefore the resource cost of an additional installation is not affected by the amount of existing capacity, and it is unnecessary for SOLES to keep track of the existing generating capacity.

SOLES is the repository of data on solar resources, costs, and technology performance characteristics. SOLES passes the fixed operating and maintenance (O\&M) costs, variable O\&M costs, and capital costs separately to both the Electricity Finance and Pricing submodule (EFP) and the Electricity Capacity Planning (ECP) submodule of the EMM. The construction leadtime in years, as well as the fraction of capital costs in the $\mathrm{j}^{\text {th }}$ year of construction, are passed to both the ECP and EFP. SOLES also reflects technological improvements in the cost and performance data. The scope of this modeling is of centralized, supply-side solar resources. Off-grid PV applications are modeled as demand-side technologies.

ST technologies concentrate direct sunlight onto a receiver where the energy absorbed by a working fluid powers a thermal conversion-generator device. PV technologies use silicon or some other semiconductor material to directly convert sunlight into electricity in proportion to the quantity of sunlight received. Unlike both ST and conventional electric generation technologies, PV has no minimum system size. Consequently, PV technology is better suited than ST technology for remote-site applications that are not served by the electricity grid.

\section{Relationship of the Solar Submodule to Other Models}

The Renewable Fuels Module (RFM), of which SOLES is a part, transmits cost and performance values for solar thermal and photovoltaic technologies to the EMM. For each forecast year, SOLES receives the cumulative solar thermal or PV capacity through the prior year, in order to produce revised estimates of capital costs. These calculations will then be provided to the RFM 
for overall renewable fuels accounting and reporting purposes. SOLES will pass capital cọsts, fixed and variable O\&M costs, construction profile, and solar availability to the EMM.

Generally, SOLES is not a part of the NEMS convergence routine because it does not provide prices or quantities to the NEMS Integrating Module (IM). Instead, it provides projections of cost and performance characteristics to the EMM, which provides the IM with electricity prices for convergence-testing.

The IM provides control information to SOLES. It supplies information on the model start and current year. It also indicates when convergence has been reached. No data are expected from the Macroeconomic Activity Module (MAM) by SOLES.

\section{Modeling Rationale}

\section{Theoretical Approach}

Solar energy supplies are fundamentally different from those for most other renewable sources. It is appropriate to model other renewables such as wind, geothermal, biomass, and hydro, which consist of limited quantities of high-quality resources, with supply functions which are upward sloping with increasing quantities demanded. In contrast, the solar resource within each EMM region for both kinds of solar technologies (ST and PV) is relatively constant for supply quantities well in excess of conceivable demand. As a result, the supply curve for solar is assumed to be flat. NEMS does not increase the cost of the resources with increasing quantities supplied, because high quality resource sites are not exhausted.

Since the two solar electric technologies generate electricity in fundamentally different ways, the nature of the solar resource for each technology is significantly different. The most important difference is the nature of the solar radiation (insolation) that each technology uses. ST technology can utilize only direct normal insolation while PV can utilize both direct and diffuse insolation. Direct normal insolation is defined as sunlight arriving at a location in a path directly from the sun onto a surface without being scattered or reflected. Diffuse insolation is sunlight that has been scattered by clouds, fog, haze, dust, or other substances in the atmosphere and arrives at a location indirectly. The sum of direct normal and diffuse insolation is also referred to as global insolation.

Initially, data have been developed for a single type of each of the ST and PV technologies to be used for all regions. Accordingly, capital and O\&M costs and the efficiency in converting sunlight into. electric energy are held constant across regions. Any differences in regional resources are captured through the variable that represents the solar energy input to the technology.

ST technologies are composed of concentrators which can only use direct normal radiation. This resource is much more sensitive to atmospheric conditions than total (direct plus diffuse) solar 
radiation. Accordingly, for solar thermal, data are provided only for 6 of the 13 EMM Regions which have sufficiently intense insolation of this kind.

The default solar thermal electric technology is a central receiver with 3-hour molten salt thermal storage. The resource data incorporate climatological data on the frequency and duration of cloud cover. The resource availability or energy output data for central receiver solar thermal consist of both daytime and evening values for the four seasons for a total of eight values. Since the number of overcast days can exceed the storage capacity of the system, a derating factor is included to reflect this intermittent availability.

The default PV technology is a flat-plate array with one tracking north-south axis tilted at an angle equal to the site's latitude. The availability or energy output is represented as four values representing the average hourly output per unit of system capacity during daytime hours for each of the four seasons defined by the load duration curve of the EMM.

\section{Fundamental Assumptions}

The regional classification plan is the same for both ST and PV.. As an input to EMM, SOLES will operate on the same 13 regions plus Alaska and Hawaii. These correspond to the nine NERC Regions with New York separated from New England; Florida separated from the rest of the Southeast; and the West separated into three regions consisting of California and Southern Nevada (CNV), the Northwest Power Pool Area (NWP), and the combination of the Rocky Mountain and Arizona-New Mexico Power Areas (RA): Each region will have its own resource data for both ST and PV, where applicable.

\section{Alternative Approaches}

Solar technologies have not often been incorporated in national-level energy models. Three exceptions are the Integrated Planning Model (IPM) from ICF Resources, FOSSIL2 from Applied Energy Services (AES) which was utilized by DOE in the 1991 National Energy Strategy (NES), and the MARKAL Model from Brookhaven National Laboratory.

The IPM is the electricity model within the Electric and Gas Utility Modeling System (EGUMS) which was developed under a joint effort of ICF Resources and RCG/Hagler; -Bailly, Inc. It can also be run in a stand-alone mode. EGUMS was also used by EPA for the analysis of greenhouse gas emission policies. Like the NEMS-EMM capacity planning submodule, IPM is a linear program that derates the capacity of a technology by multiplying its rated capacity by its availability factor. IPM uses a regionalization scheme, similar to EMM, based on 11 NERC regions and subregions. IPM credits the intermittent resources on an hourly basis before creating a loạd duration curve and solving for an inter-temporal optimum. However, IPM does not try to incorporate the effect of experience or learning on the cost of the technology: As an emerging technology, solar is expected to experience sharp reductions in cost with additional penetrations so varied representations of "learning-by-doing" are certainly important in solar modeling. 
FOSSL2's fundamentally different structure uses system dynamics with logit functions adapted from consumer choice methodologies. As a system dynamics model, FOSSL2 uses difference equations to simulate the evolution of a system by taking steps, (four per year) through time rather than an optimization approach. The model compares the marginal costs of new technologies and chooses the least cost option according to a logit function that prevents knifeedge solutions. This ådjustment is particularly important because FOSSIL2 treats the entire nation as a single region.

In modeling renewables, FOSSIL2 does distinguish between dispersed and centralized electricity generation and between utility and nonutility generators (NUGS). However, in treating intermittent resources, FOSSIL 2 has difficulty because it implicitly assumes that intermittent and dispatchable technologies are supplying identical services. Intermittents are treated the same as conventional baseload technologies which gives intermittents a capacity credit equal to their rated capacity and overstates their contribution. On the other hand, FOSSIL 2 does not capture the correlation of solar with load which increases its energy value. A later version of FOSSIL2, named IDEAS, does capture this correlation by giving it more output during peak periods. This is accomplished by forcing an appropriate percentage of the intermittent resource's output into the peak portion of the load duration curve.

As discussed earlier, an important capability when modeling renewables is how new technology penetration is treated. FOSSIL2 applies a premium to the discount rate when calculating marginal cost. While the premium diminishes with cumulative production to reflect increased penetration, FOSSIL2 does not embody the notion of "learning-by-doing" and industry acceptance of new technologies.

By. contrast, in the AEO Forecasting System, solar penetration was modeled as an off-line analysis; with projected capacity and capacity factors provided to the electricity module. Generation from solar was assumed to reduce the demand for other sources of electricity generation. The projections of capacity additions and capacity factors, also off-line; are based on planned additions as reported by electricity producers to EIA and utilization factors are based on the most recent data and trends. Unplanned capacity additions are derived from the NES and renewables "white paper".projections [SERI, 1990]. The AEEO Forecasting System provides projections for both utilities and NUGS by the ten Federal Regions through 2010.

The third modeling system for solar-based electricity generation is the MARKAL model, developed, in part, at Brookhaven National Laboratory with the support of 17 nations and two international agencies. MARKAL is a multi-period, linear-programming model that performs energy systems optimization and addresses all aspects of the energy system. Its primary objective is to assess the attractiveness of existing and new energy technologies and resources in satisfying future demand. Within the energy network, the model user has total control over the level of technology detail. However, MARKAL cannot easily or transparently incorporate non-price-based consumer choices or new technology penetration. 


\section{Solar Submodule Structure}

\section{Submodule Flow Diagram}

-A flow diagram showing the main computational steps and relationships of the Solar Submodule is shown in Figure 3.

Figure 3. Solar Energy Submodule Flowchart

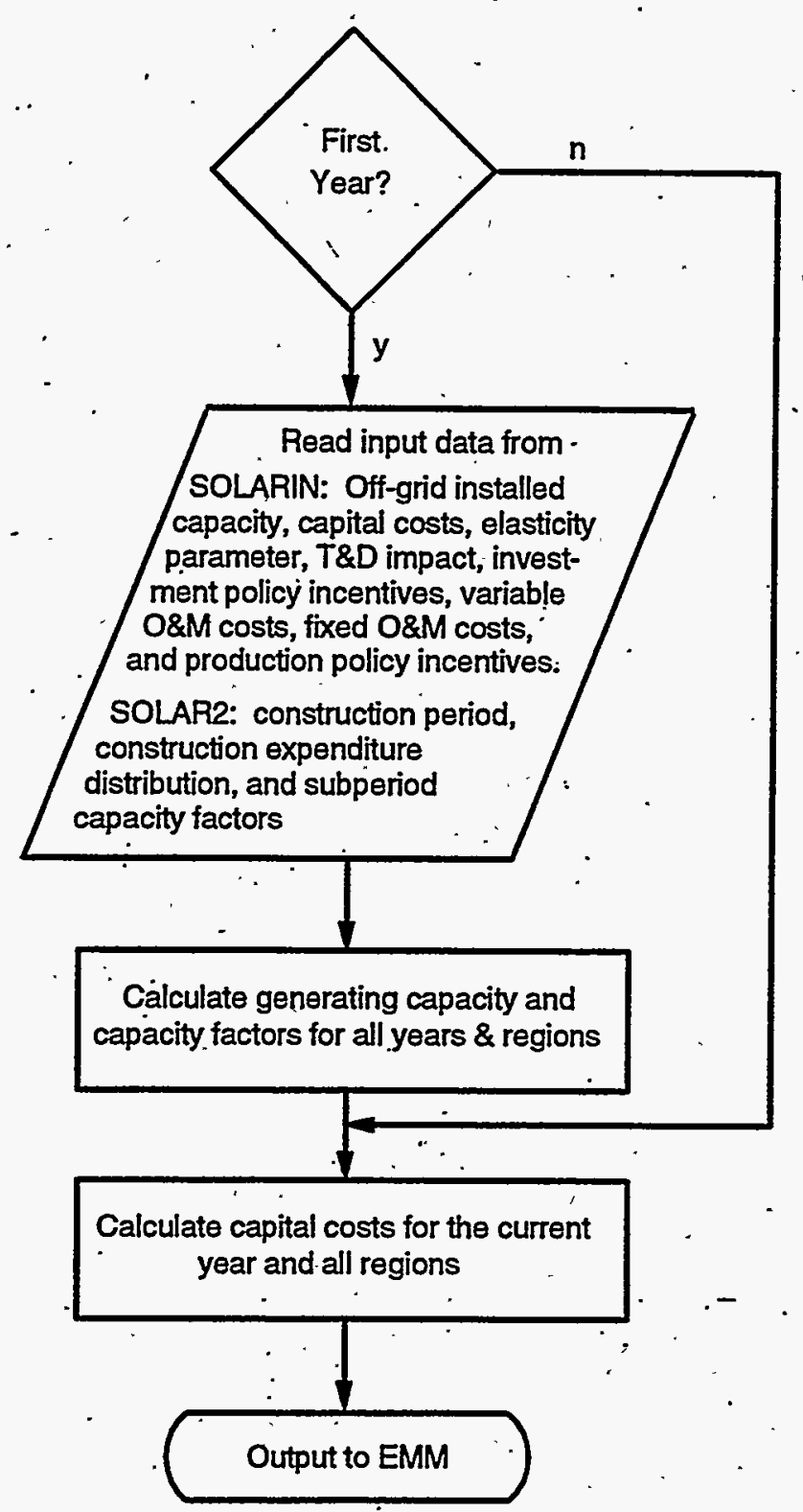




\section{Key Computations and Equations}

In its current embodiment, SOLES passes data directly, without any computations, through assignments to the appropriate COMMON variables. These are the utility generating capacities and capacity factors, capital costs; O\&M costs, and subperiod capacity factors for each technology. The variable O\&M costs for photovoltaics are converted from $\$ / \mathrm{kWh}$ to mills $/ \mathrm{kWh}$ before assigtment to the COMMON variable. 


\section{Appendix 4-A: Inventory of Variables, Data, and Parameters}

This Appendix describes the variables, data inputs, and parrameter estimates associated with the Solar Submodule. Table 4A-1 provides a tabular listing of model variables and parameters. The table contains columns with information on item definitions, modeling dimensions, data sources, measurement units, and documentation page references.

The remainder of Appendix 4-A consists of detailed descriptions of data inputs and variables, including discussions on supporting data assumptions and transformations.

Table 4A-1. NEMS Solar Model Inputs and Outputs.

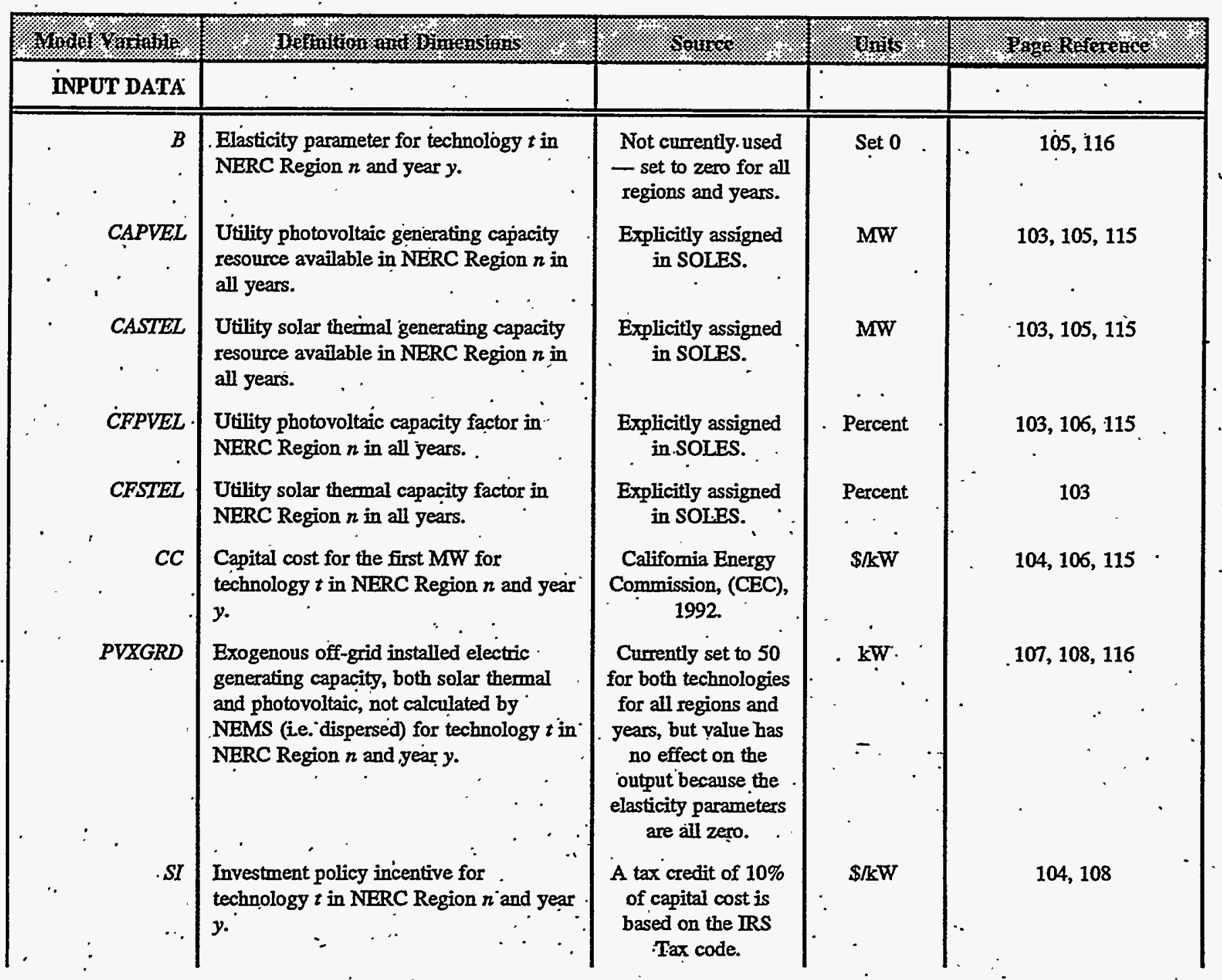


Tảble 4A-1. NEMS Solar Model Inputs and Outputs (Continued)

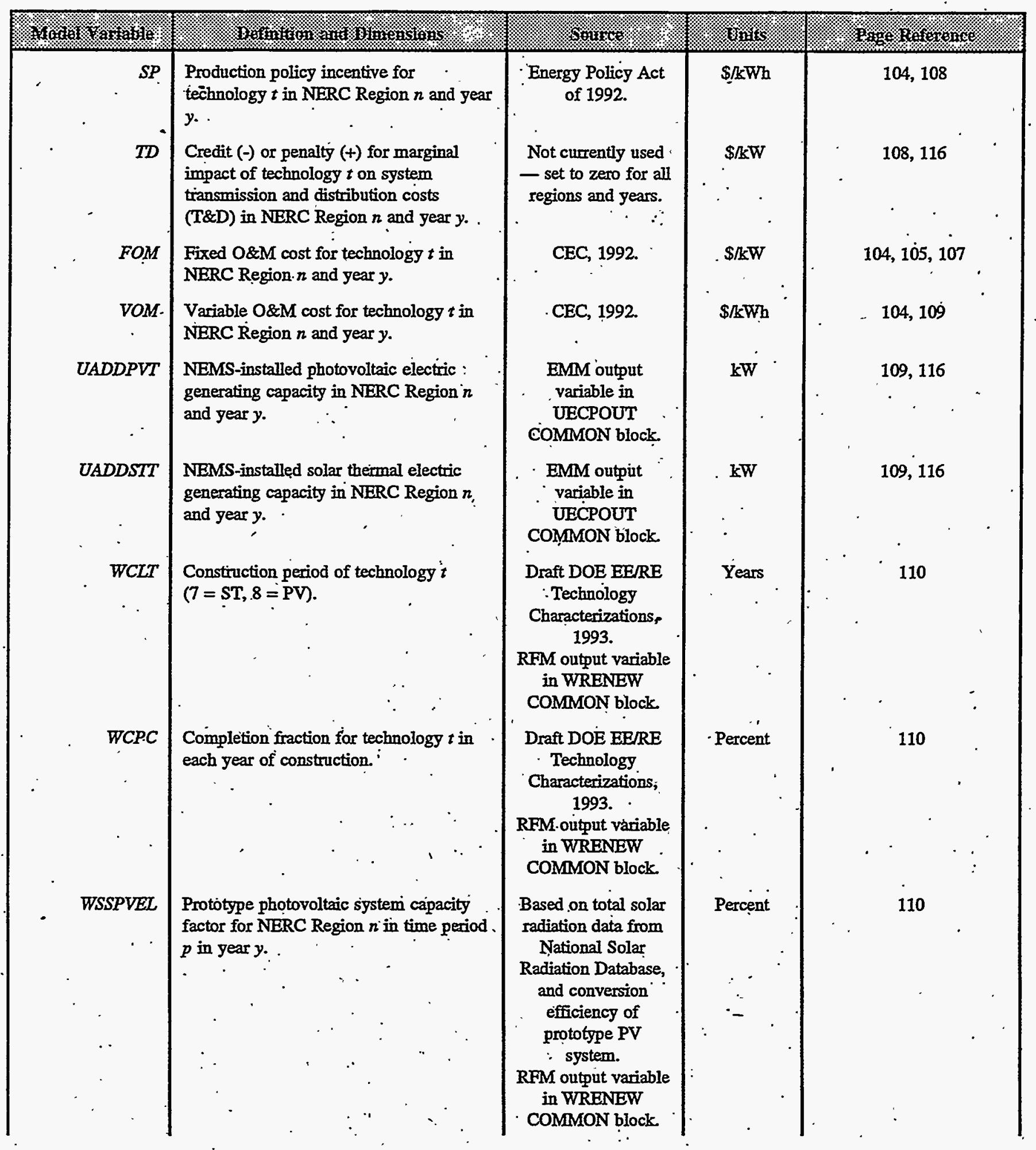


Table 4A-1. NEMS Solar Model Inputs and Outputs'(Continued)

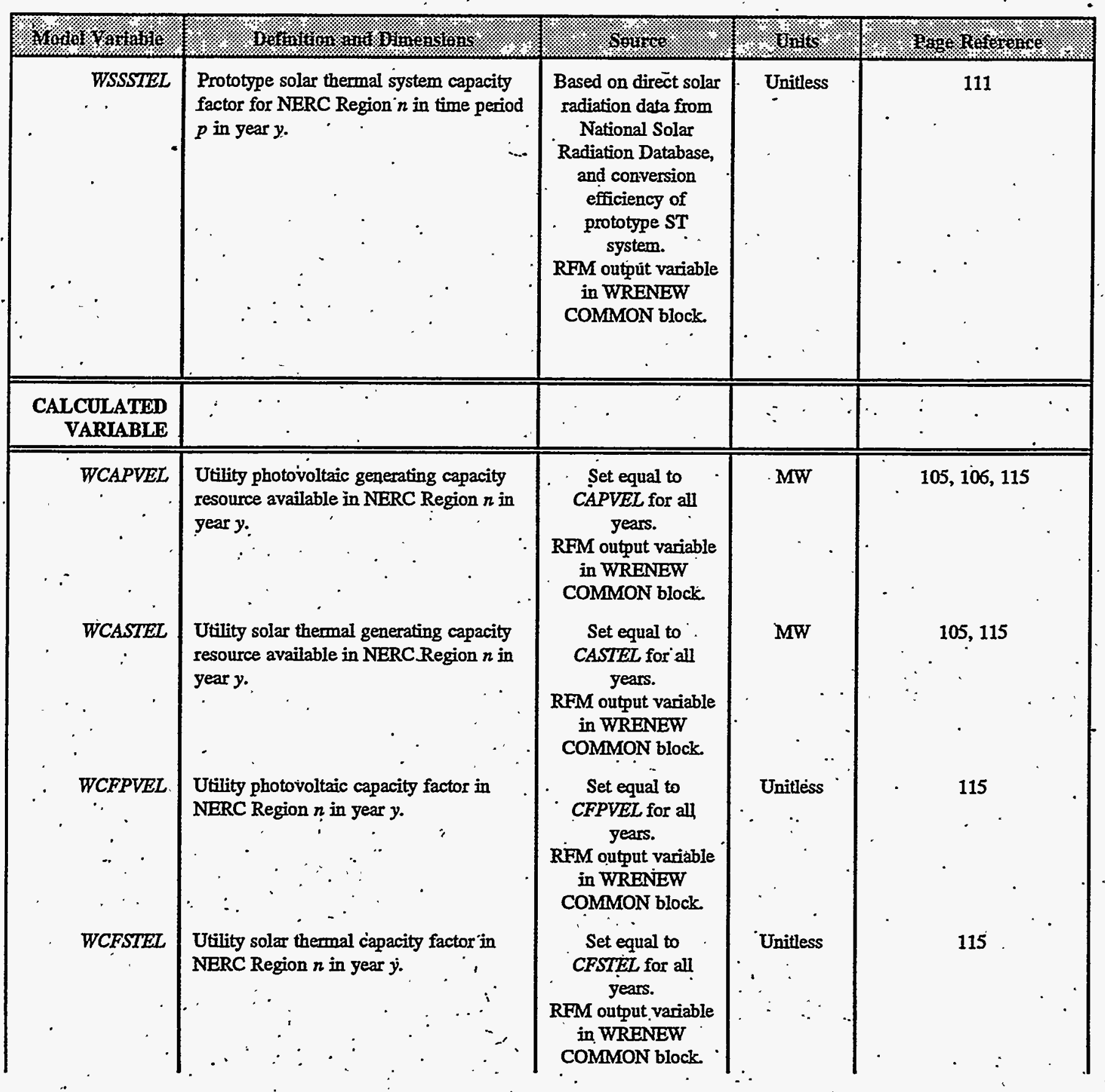


Table 4A-1. NEMS Solar Model Inputs and Outputs (Continued)

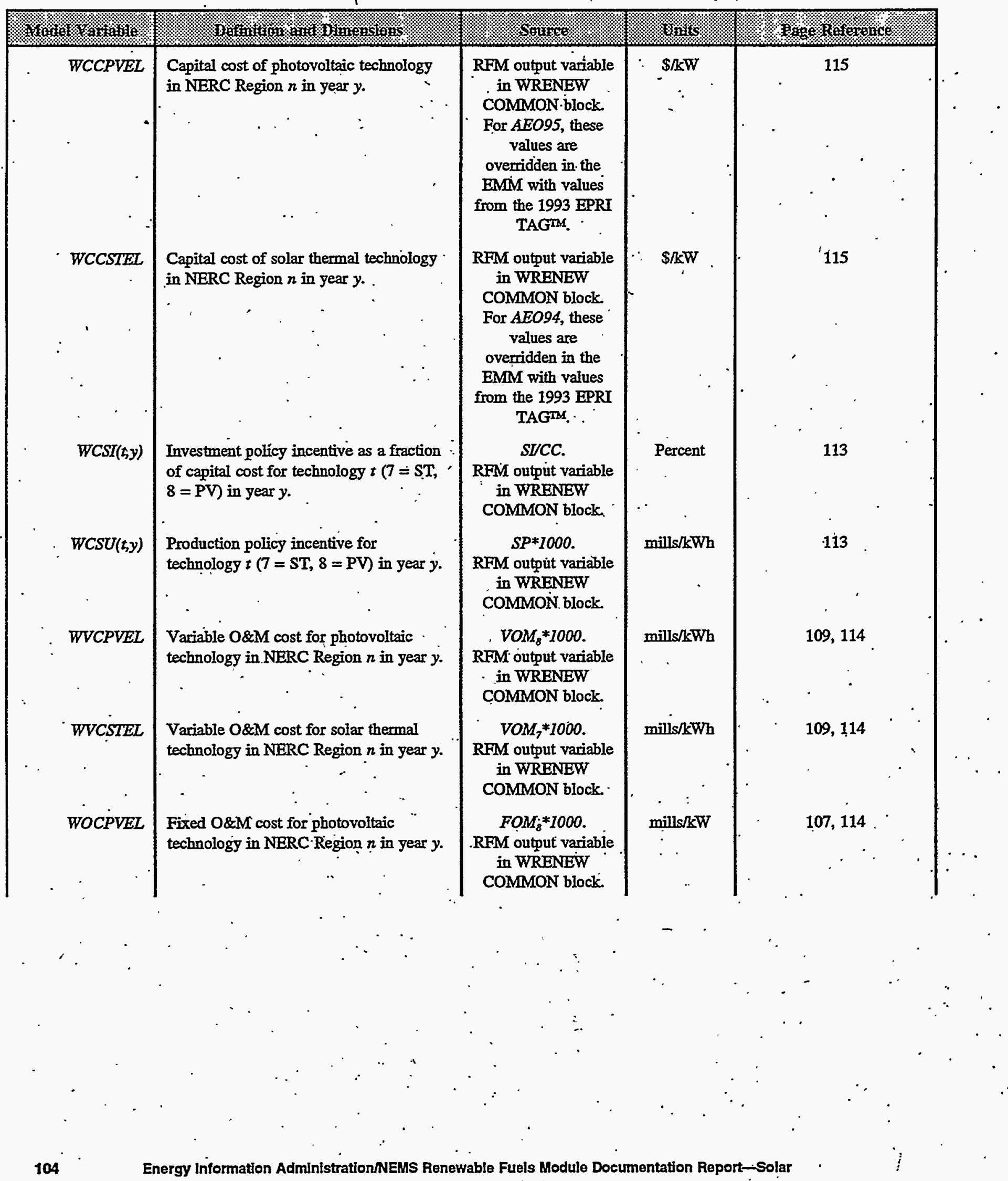


Table 4A-1. NEMS Solar Model Inputs and Outputs (Continued)

\begin{tabular}{|c|c|c|c|c|}
\hline 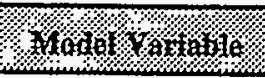 & 4. & 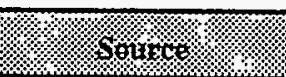 & (16isis: & (1) \\
\hline WOCSTEL & $\begin{array}{l}\text { Fixed O\&M cost for solar thermal } \\
\text { technology in NERC Region } n \text { in year } y \text {. } \\
\text {. }\end{array}$ & $\begin{array}{l}\text { FOM } \\
\text { RFM output variable } \\
\text { in WRENEW } \\
\text { COMMON block. }\end{array}$ & mills $/ \mathrm{kW}$ & 107,114 \\
\hline
\end{tabular}

\section{MODEL INPUT: $\quad B$}

DEFINITION:

Elasticity parameter for technology $t$ in NERC Region $n$ and year $y$ (Unitless).

The model is coded to use the elasticity parameter to reduce the capital costs of the technologies over time due to the "learning curve". effect. However, in the current input file, \&6005PRJ.RENEW.SOLARIN.BASE,D0914931, the value of the elasticity parameter is set to zero for both technologies for all NERC Regions and all years. As a result, the calculated capital costs are equal to the values in the input file. These values are the same for-all regions.

SOURCE: Not applicable; the currently set value (0) results in a multiplier with a value of "1."

\section{MODEL INPUT: CAPVEL}

DEFINITION: Utility photovoltaic generating capacity resource available in NERC
Region $n$; (MW).

A value of 999 for all regions is set in program code. This value is transferred to the common variable. WCAPVEL for all years. The value of 999 . corresponds to a very large value, reflecting the resource availability that is essentially limitless in comparison with conceivable demand.

SOURCE: . National Renewable Ėnergy Laboratory.

\section{MODELL INPUT: $\quad \dot{C} A S T E L$}

DEFINITION: - Utility solar thermal generating capacity resource availability in NERC Region $n$; (MW).

A value of 999 is set in the program code for six all regions of solar thermal applicability and a value of zero is set for all other regions. These values are transferred to the common variable WCASTEL for all years. The value of 999 corresponds to a very large value, reflecting the resource availability that is essentially limitless in comparison with conceivable demand. 
SOURCE: National Renewable Energy Laboratory.

\section{MODEL INPUT: CFPVEL}

DEFINITION: - Utility photovoltaic capacity factor in NERC Region $n$; (Percent).

Values are set in the program code as follows:

Table 4A-2. Selected Photovoltaic Capaciły Factor by Modified NERC Region

\begin{tabular}{|l|l|l|l|l|l|l|l|l|l|l|l|l|l|l|l|l||}
\hline Region & 1 & 2 & 3 & 4 & 5 & 6 & 7 & 8 & 9. & 10 & 11 & 12 & 13 & 14 & 15 & 16 \\
\hline CFPVEL & .15 & .15 & .15 & .15 & .20 & .15 & .15 & .15 & .15 & .20 & .20 & .20 & .20 & 0 & 0 & 0 \\
\hline
\end{tabular}

These values are transferred to the common variable WCFPVEL for all years.

SOURCE: . National Renewable Energy Laboratory.

MODEL INPUT: : CFSTEL

DEFINITION: - Utility solar thermal capacity factor in NERC Region $n$; (Percent).

Values are set in the program code as follows:

Table 4A-3. Selected Solar Thermal Capacity Factors by Modified NERC Region

\begin{tabular}{|l|l|l|l|l|l|l|l|l|l|l|l|l|l|l|l|l|}
\hline Rëgion & 1 & 2 & 3 & 4 & 5 & 6 & 7 & 8 & 9 & 10 & 11 & .12 & 13 & 14 & 15 & 16 \\
\hline CFSTEL $_{2}$ & 0 & .30 & 0 & 0 & .24 & 0 & 0 & 0 & 0 & .27 & .30 & .35 & .40 & 0 & 0 & 0 \\
\hline CFSTEL $_{2}$ & 0 & .47 & 0 & 0 & .38 & 0 & 0 & 0 & 0 & .43 & .47 & .55 & .63 & 0 & 0 & 0 \\
\hline
\end{tabular}

The values of CFSTEL $_{1}$ are transferred to the common variable WCFSTEL for years $1-18$, and the values of CFSTEL $_{2}$ are transferred to the common variable WCFSTEL for years above 18.

SOURCE: " National Renewable Energy Laboratory: 
MODEL INPUT: $\quad C C$

DEFINITION: Capital cost for the first MW for technology $t$ in NERC Region $n$ and year $y$; (\$/kW).

The capital costs for solar thermal are set at $\$ 1840 / \mathrm{kW}$ for all regions and years, based on data reported by 'the California. Energy Commission. The capital costs for photovoltaics start at $\$ 8000 / \mathrm{kW}$ in 1990 and decrease' steadily to $\$ 700 / \mathrm{kW}$ in 2030 .

SOURCES: Draft DOE EE/RE Technology Characterizations, 1993.

. The California Energy Commission, "1992 Energy Technology Status Report," December 1992.

"Data Development Report for the Solar Electric Generation Submodule (SOLES) Renewable Fuels Module National Energy Modeling System," Prepared by Peter Lilienthal; National Renewable Energy Laboratory, Draft, June 30, 1993.

Phone conservation with Peter Lilienthal, Market and Analysis Branch, NREL, December 2, 1993.

\section{MODEL INPUT: $\quad F O M$}

DEFINITION: Fixed O\&M cost for technology $t$ in NERC Region $n$ and year $y$; (\$/kW).

The fixed O\&M costs are set to zero for the SH technology for all NERC Regions and all years. The fixed O\&M costs for solar thermal are set at $\$ 27.86 / \mathrm{kW}$ for all regions and years, based on data reported by the California Energy Commission. The values are transferred to the common variables WOCPVEL and WOCSTEL for PV and ST respectively.

SOURCES: "Data Development Report for the Solar Electric Generation Submodule (SOLES) Renewable Fuels Module National Energy Modeling System," Prepared by Peter Lilienthal, National Renewable Energy Laboratory, Draft, June 30, 1993.

The California Energy Commission, "1992 Energy Technology Status Report,". December 1992.

Draft DOE EE/RE Technology Characterizations, $199 \overline{3}$.

Phone conservation with Peter Lilienthal, Market and Analysis Branch, NREL, December 2, 1993. 


\section{MODEL INPUT: . PVXGRD}

DEFINITION: - Exogenous installed electric generating capacity not calculated by NEMS (i.e., off-grid) for technology $t$ in NERC Region $n$ and year $y ;(\mathrm{kW})$.

In the current input file, \&6005PRJ.RENEW.SOLARIN.BASE.D0914931, the value is set to $50 \mathrm{~kW}$ for both technologies for all NERC Regions and all years. This variable is defined and discussed as being applicable to PV only; howiever, the model is constructed to employ the variable for both ST and PV. The negative of the elasticity parameter is an exponent on PVXGRD in the code, and the result of this exponentiation is multiplied by the capital cost. Since the elasticity parameter is set to zero in the current data file, the model output of capital cost is currently unaffected by the value of PVXGRD.

SOURCE: Irrelevant, since the currently set value of the elasticity parameter causes the value of exogenous installed electric generating capacity to have no effect on the capital costs.

\section{MODEL INPUT: ' SI}

DEFINITION: Investment policy incentive for technology $t$ in NERC Region $n$ and year $y ;(\$ / k W)$.

This is currently set at 10 percent of the capital cost, based on a 10 percent investment tax credit.

SOURCE: Energy Policy Act of 1992 (P.L. 102-486), Title 19, Section 1916.

\section{MODEL INPUT: $\quad S P$}

DEFINITION: Production policy incentive for technology $t$ in NERC Region $n$ and year $y ;(\$ / k W h)$.

A value of 15 mills per kilowatt hour for the years 1995 through 2004 and zero for all other years.is assigned for all regions, based on Section 1212 of the Energy Policy Act of 1992. The act states that the incentive is payable for ten years for a facility that first generates electricity during the ten fiscal year period occurring after enactment. Since solar thermal technology is not scheduled to occur until 2000 , it would seem appropriate to: shift this incentive for that techriology to the years 2000 through 2009.

SOURCE: Energy Policy Act of 1992, Public Law 102-486, Section 1212. 


\section{MODEL INPUT: TD}

DEFINITION: Credit or penalty for impact of technology $t$ on system T\&D in NERC Region $n$ and year $y ;(\$ / k W)$.

In the current input file, \&6005PRJ.RENEW.SOLARIN.BASE.D0914931, the value is set to zero for both technologies for all NERC Regions and years.

SOURCE: Irrelevant, since the currently set values have no effect on the capital costs.

\section{MODEL INPUT: $\quad U A D D P V T$}

DEFINITION: NEMS-installed photovoltaic electric generating capacity in NERC Region $n$ and year $y$; (kW).

SOURCE: EMM output variable in ÜECPOUTT COMMON block.

\section{MODEL INPUT: $\quad U A D D S T T$}

DEFINITION: . NEMS-installed' solar thermal electric generating capacity in NERC Region $n$ and year $y ;(\mathrm{kW})$.

SOURCE: EMM output variable in UECPOUT COMMON block.

\section{MODEL INPUT: VOM}

DEFINITION: . Variable O\&M costs for technology $t$ in NERC Region $n$ and year $y$

The variable O\&M costs for the ST technology are set to zero for all NERC Regions and all years, based on data reported by the California Energy Commission. The variable O\&M costs for the PV technology. are set at 2 mills $/ \mathrm{kWh}$ for the years 1990 through 2001 . In 2002 these jump to $5.4 \mathrm{mills} / \mathrm{kwh}$ due to a change from crystalline silicon to thin film technology, after which they decrease steadily to $1 \mathrm{mill} / \mathrm{kWh}$ in 2020 and beyond. The -values are transferred to the common variables WVCPVEL and WVCSTEL for PV and ST respectively.

SOURCES: "Data Development Report for the Solar Electric Generation Submodule (SOLES) Renewable Fuels Module National Energy Modeling System," prepared by Peter Lilienthal, National Renewable Energy Laboratory, Diaft, June 30, 1993.

The California.Energy Commission, "1992 Energy Technology Status Report," December 1992.

Draft DOE EE/RE Technology. Characterizations, 1993. 
Phone conservation with Peter Lilienthal, Market and Analysis Branch, NREL, December 2, 1993.

\section{MODEL INPUT: : WCLT}

DEFINITION: Construction period of technology $t$, where $t=7$ corresponds to ST and $t=8$ corresponds to $\mathrm{PV}$; (Years).

This variable is read from the input.file directly into the.WRENEW COMMON block.

SOURCES: Pacific Gas and Electric Co., Solar Central Receiver Technology Advancement for - Electric UUtility Applications, September 1988.

Draft DOE EE/RE Technology. Characterizations, 1993.

\section{MODEL INPUT: $\quad$ WCPC}

DEFINITION: Fraction of construction of technology $t$ completed in year $y$; (Percent).

This variable is read from the input file directly into the WRENEW COMMON block.

SOURCES: Pacific Gas and Electric Co.; Solar Central Receiver Technology Advancement for Electric Utility Applications, September 1988.

Draft DOE EE/RE Technology Characterizations, 1993.

\section{MODEL INPUT: · WSSPVEL}

DEFINITION: Prototype photovoltaic system capacity factor for NERC Region $n$ in time period $p$ in year $y$; (Percent).

This variable is read from the input file directly into the WRENEW COMMON block.

- SOURCE: Based on total solar radiation data from National Solar Radiation Database, and conversion efficiency of prototype $\mathbb{P V}$ system used in Draft DOE EE/RE Technology Characterizations, 1993. 
MODEL INPUT: WSSSTEL

DEFINITION: 'Prototype solar thermal system capacity factor for NERC Region $n$ in time period $p$ in year $y$; (Unitless).

This variable is read from the input file directly into the WRENEW COMMON block.

SOURCE: Based on total solar radiation data from National Solar Radiation Database, and conversion efficiency of prototype ST system used in. Draft DOE EE/RE Technology Characterizations, 1993. 



\section{Appendix 4-B: Mathematical Description}

This Appendix provides the detailed mathematical specification of the Solar Submodule as presented in the SOLES FORTRAN code execution sequence. Subscript definitions are also as they appear in the FORTRAN code. Within SOLES a technology subscript " $t$ " is used. For solar thermal, $t=7 ;$ for $P V, t=8$.

The investment policy incentives are converted to a fraction of the capital costs according to:

$$
W C S I_{t y}=S I_{n, y y} l_{y} C C_{n, y y}
$$

where:

$$
\begin{aligned}
\dot{W C S I}_{t, y}= & \text { Investment policy incentive as a fraction of capital cost for technology } t \\
& (7=\mathrm{ST}, 8=\mathrm{PV}) \text { in year } y, \\
\vdots & =
\end{aligned}
$$

The production policy incentives are converted to mills/kWh according to:

$$
W C S U_{t, y}=1000 \times S P_{t y}
$$

where:

$W C S U_{k y}=$ Production policy incentive as a fraction of capital cost for technology $t$ . $(7=\mathrm{ST}, 8=\mathrm{PV})$ in year $y$,

$\dot{S P}_{t, y} \quad=$ Production policy incentive for technology $t$ in year $y ;$ set $=0$

The solar thermal O\&M costs, in mills per $\mathrm{kWh}$, are assigned according to:

$$
\begin{aligned}
& \text { WVCSTEL } L_{n, y}=1000 \times V O M_{n, 7 y} \\
& \text { WOCSTEL } \\
& \text { W }_{n, y}=1000 \times F_{n, 7 y}
\end{aligned}
$$


where:

WVCSTEL $_{n, y}=$ Variable O\&M cost for solar thermal technology in NERC Region $n$ in year $y$;

WOCSTEL $L_{n y}=$ Fixed O\&M cost for solar thermal technology in NERC Region $n$ in year $y$,

$V O M_{m, y}=$ Variable O\&M cost for solar thermal technology in NERC Region $n$ in year $y$,

FOM $_{n, 7, y}=$ Fixed O\&M cost for solar thermal technology in NERC Region $n$ in year $y$.

The photovoltaic O\&M costs are assigned according to:

$$
\begin{aligned}
& \text { WVCPVEL } L_{n, y}=1000 \times V O M_{n, 8,} \\
& \text { WOCPVEL }_{n, y}=1000 \times F O M_{n, 8, y}
\end{aligned}
$$

where:

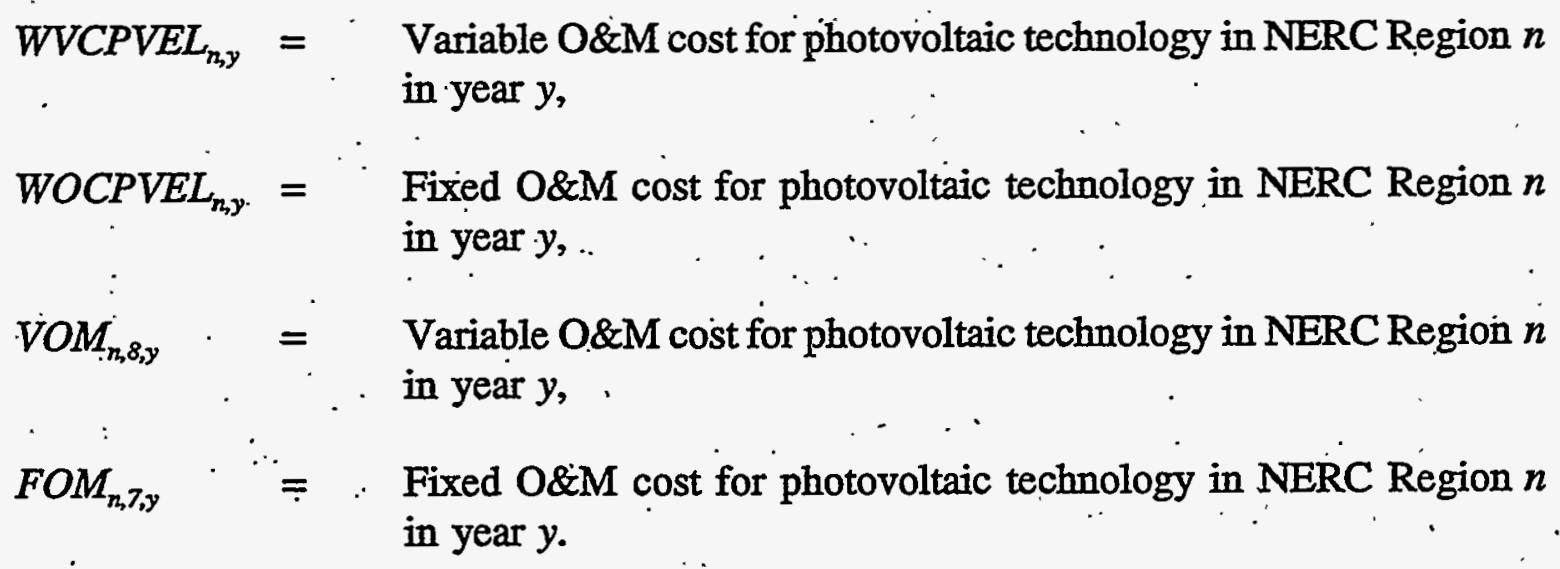


The utility generating capacities and capacity factors are calculated according to:

$$
\begin{aligned}
& \text { WCASTEL }_{n, y}=\text { CASTEL }_{n} \\
& \text { WCAPVEL }_{n y}=C A P V E L_{n} \\
& \text { WCFSTEL }_{n, y}=\text { CFSTEL }_{n, 1} \quad \text { when } y \leq 18 \\
& \text { WCFSTEL }_{n, y^{-}}=\text {CFSTEL }_{n, 2} \text {. when } y \geq 19 \\
& \text { WCFPVEL }_{n j}=\text { CFPVEL } L_{n}
\end{aligned}
$$

where:

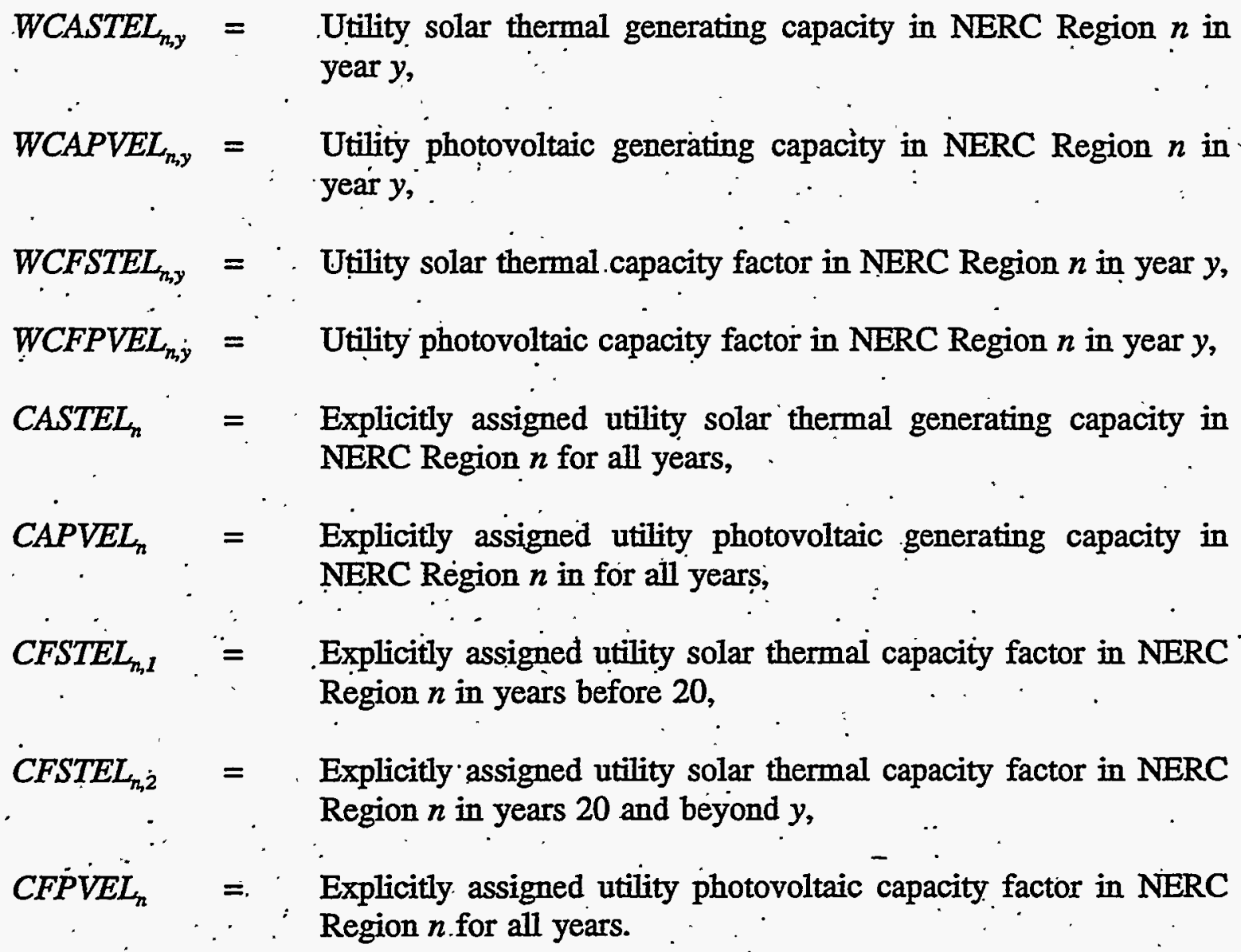


The capital costs are calculated according to:

$$
\begin{aligned}
& \text { WCCSTEL } L_{n, y}=C C_{n, 7, y} \times P^{B_{n, 7 y}}+T D_{n, 7 y} \\
& W C C P V E L_{n, y}=C C_{n, 8, y} \times P^{B_{n, 8 y}}+T D_{n, 8, y}
\end{aligned}
$$

where:

$$
\begin{aligned}
& \text { WCCSTEL } L_{n y} \doteq \text { Capital cost of solar thermal technology in NERC Region } n \text { in } \\
& \text { year } y \text {, } \\
& \text { WCCPVEL }_{m, y}=\text { Capital cost of photovoltaic technology in NERC Region } n \text { in } \\
& \text { year } y \text {, } \\
& C C_{n, t y} \quad, \quad=\quad \text { Capital cost for - the first MW of technology } t(7=\mathrm{ST}, 8=\mathrm{PV}) \text { in } \\
& \text { NERC Region } n \text { in year } y \text {, } \\
& \begin{array}{l}
P_{n, 7, y}=\sum_{j=1}^{y}\left(P V X G R D_{n, 7, j}+\sum_{i=1}^{n} U_{A D D S T T_{i, j}}\right) \\
P_{n, 8, y}=\sum_{j=1}^{y}\left(P V X G R D_{n, 8, j}+\sum_{i=1}^{n} U A D D P V T_{i, j}\right)
\end{array}
\end{aligned}
$$

where:

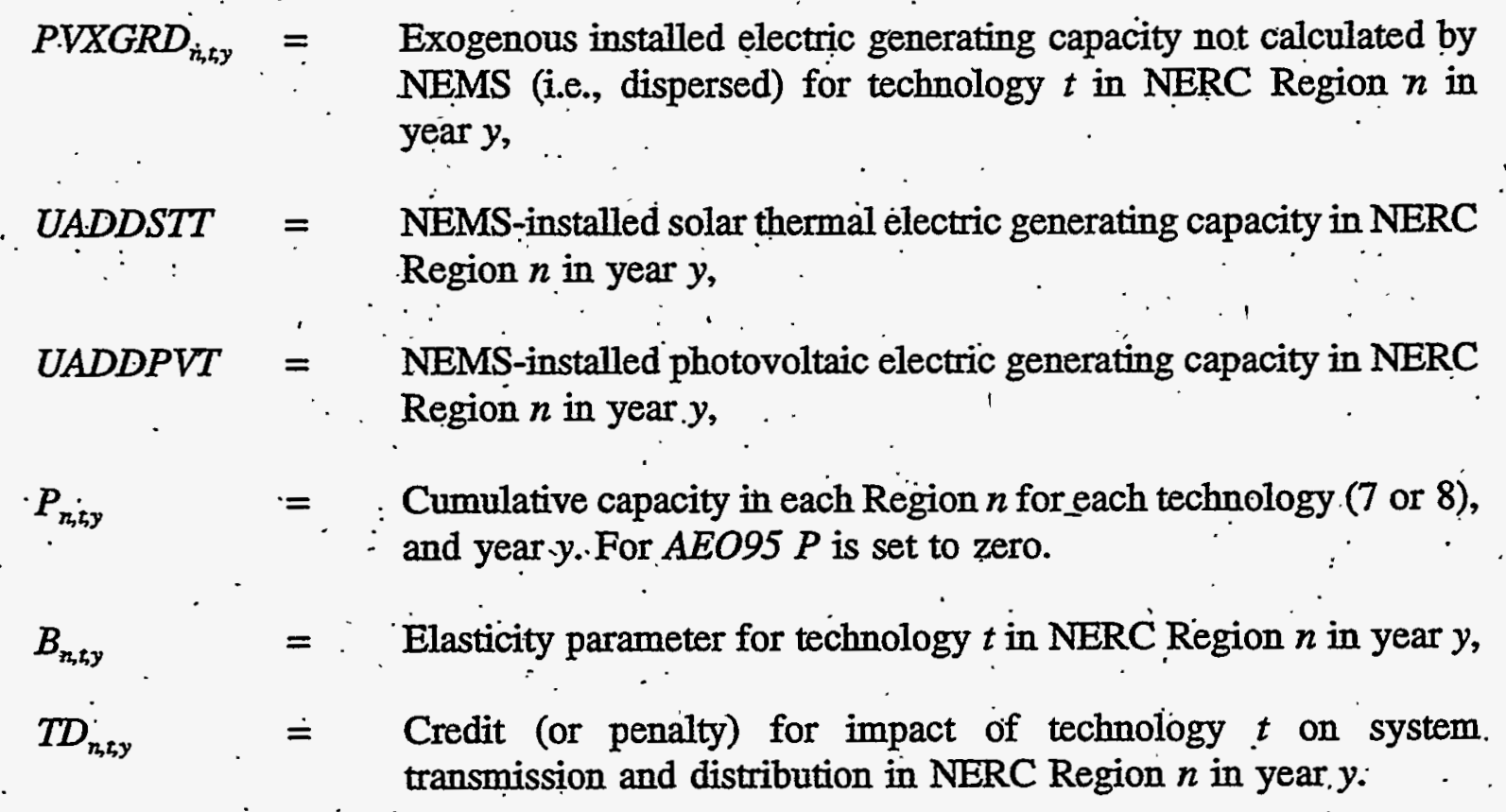


Note that, because the values of B and TD in the current input file are zero for all regions and years, the capital costs are transferred without adjustment from the input file to the respective COMMON variables, i.e.:

$$
\begin{aligned}
& \text { WCCSTEL }_{n, y}=C C_{n, 7, y} \\
& \text { WCCPVEL } \\
& \text { nCy }_{n, y}=C C_{n, y}
\end{aligned}
$$





\section{Appendix 4-C: Bibliography}

California Energy Commission, "1992 Energy Technology Status Report," December 1992.

Energy Information Administration, "Component Design Report for Solar and Wind Submodules Renewable Fuels Module National Energy Modeling System," Draft, April 23, 1993.

Krawiec, Frank and Flaim, Theresa, "Solar Cost Reduction Through Technical Improvements, . The Concept of Learning and Experience," SERI/RR-52-173, October 1979.

Lilienthal, Peter,. "Data Development Report for the' Solar Electric Generation Submodule (SOLES) Renewable Fuels Module National Energy Modeling System," NREL, Draft, June 30; 1993.

Menicucci, D.F., and Fernandez, J.P. "Estimates of Available Solar Radiation and Photovoltaic Energy Production for Various Tilted and Tracked Surfaces Throughout the US Based on -PVFORM, a Computerized Performance Model," Sand 85-2775, Sandia National Laboratories, Albuquerque, NM 87185.

National Renewable Energy Laboratory, "National Solar Radiation' Data Base (1961 - 1990) Version 1.0 NSRDB-Volume 1, September, 1992," Prepared by NREL, distributed by National Climatic Data Center, Asheville, NC 28801.

National Renewable Energy Laboratory, "Photovoltaics Program Plan FY 1991 - FY 1995," NREL, Golden, CO, DOE/CH10093-92 DE91002139.

Pacific Gas and Electric Co., "Solar Central Receiver Technology Advancement for Electric Utility Applications," September 1988.

Solar Energy Research Institute. "The Potential of Renewable Energy: An Interlaboratory White Paper," SERI/TP-260-3674, Golden, CO, prepared for the Office of Policy, Planning, and Analysis, U.S. Department of Energy, in support of the National Energy Strategy, March 1990. 



\section{Appendix 4-D: Model Abstract}

Model Name:

Solar Submodule .

Model Acronym:

SOLES

\section{Description:}

SOLES provides projections of costs and performance characteristics (including resource availabilities) for photovoltaic and solar thermal electricity generating systems by EMM region and year. EMM Regions are based on the North American Electric Reliability Council (NERC) 'Regions as modified by the Energy Information Administration (EIA) for NEMS. For PV technologies, all EMM Regions are represented in SOLES. For ST technologies, however, only six selected regions are represented, since this technology will not compete in all regions of the country.

\section{Purpose of the Model:}

The purpose of the NEMS Solar-Electric Submodule (SOLES) is to project the costs and performance characteristics of Solar Thermal (ST) and Photovoltaic (PV) electricity generating technologies, and provide them to the Electric Market Module (EMM) for dispatching these technologies in competition with other sources of electricity generation. Projections of costs and performance characteristics (including resource availabilities) are provided by EMM Region and year.

\section{Most Recent Model Update:}

November 3, 1994

\section{Part of Another Model?:}

The Solar Submodule is a component of the Renewable Fuels Module (RFM) of the National Energy Modeling System (NEMS).

\section{Official Model Representative:}

Thomas Petersik

Coal, Uranium, and Renewable Fuels Analysis Branch

Energy Information Administration

(202) 586-6582

\section{Documentation:}

NEMS Documentation Report: Renewable Fuels Submodule, May 1995.

\section{Archive Media and Installation Manual(s):}

Archived as.part of the NEMS production runs. 


\section{Energy System Described:}

Solar thermal performance is based on a central receiver system with molten salt storage. The storage allows the electricity output to be dispatched over a somewhat longer period than hours of highest.... it is "decoupled." At low levels of insolation the output of the central receiver system is zero. Once the insolation exceeds a threshold level sufficient to overcome thermal losses, the daily total output is assumed to be linear with total daily insolation. The output is allocated first to day periods, then to evening periods, and then to nighttime periods. Photovoltaic performance is based on a north-south axis tracking PV system. The technology characterization assumes that rated output is reached at an insolation level of 1000 Watts per square meter.

\section{Coverage:}

- Geographic: 15 NERC Regions: East Central, Texas, Mid-Atlantic, Mid-America, MidContinent, Northeast, New England, Florida, Southeastern, Southwest, Western, Rocky mountain \& Arizona, California \& So. Nevada, Alaska, and Hawaii

- . Time Unit/Frequency: Annual, 1990 through 2015

$\therefore \quad$ Products: Electricity.

\section{Modeling Features:}

\section{Non-DOE Input Sources:}

Pacific Gas and Ėlectric Co.

- Capital costs for ST technology

- Fixed O\&M costs for ST technology

-. Variable O\&M costs for ST technology

- Construction period for ST technology

- Construction fraction distribution for.ST technology.

Energy Policy Act of 1992

- Production policy incentive.

IRS Tax Code

- .10 percent investment tax credit.

National Solar Radiation Database .

- Regional Insolation 


\section{DOE Input Sources:}

EE/RE Technology Characterizations

- Capital costs for PV technology

- Fixed O\&M costs for PV technology.

- Variable O\&M costs for PV technology

- Prototype system efficiencies

- Construction period for:PV technology

- : Construction fraction distribution for PV technology.

\section{Computing Environment:}

- Hardware Used: IBM 3090

- Operating System: MVS

- Language/Software Used: VS FỌRTRAN, Ver. 2.05

- Memory Requirement: $19 \mathrm{~Kb}$

- Storage Requirement: $410 \mathrm{~Kb}$

- Estimated Run Time: .03 seconds

- Special Features: None.

\section{Independent Expert Reviews Conducted:}

None.

\section{Status of Evaluation Efforts by Sponsor:}

None. 


\section{Appendix 4-E: Data Quality and Estimation Processes.}

This Appendix discusses (1) the quality of the principal sources of input data used in the Solar Submodule, along with a discussion of user-defined parameters and guidelines used to select them, and (2) estimation methods used to derive parameters.

\section{Solar Thermal Performance}

Solar thermal performance (the derating factors) is based on a central receiver system with molten salt storage. The storage allows the electricity output to.be dispatched at any time of day, i.e., it is "decoupled" from the periods of high insolation. Because it uses concentrators, the central receiver system can utilize only direct insolation. At low levels of insolation the output of the central receiver system is zero. Once the insolation exceeds a threshold level sufficient to overcome thermal losses, the daily total output is assumed to be linear with total daily insolation. The output is allocated first to the day period. On days when there is more insolation than can be converted in the day period, output is delivered in the evening period. Similarly, when the daily insolation cannot all be converted during the day and evening periods, output is delivered in the night-time period. Two different output functions are used because it is expected that after experience is gained with early installations, additional collectors and storage will be used to increase the central receiver system output at a given insolation level. This change is assumed to occur in 2008. This procedure is followed for one representative site in each region.

\section{Photovoltaic Performance}

Photovoltaic performance is based on a north-south axis tracking PV system. The technology characterization assumes that rated output is reached at an insolation level of 1000 Watts per square meter. The fraction of rated capacity of the PV system is assumed to vary linearly with (direct plus diffuse) insolation, so that at any instant the capacity is equal to the insolation in $\mathrm{W} / \mathrm{m}^{2}$ divided by 1000 . PV O\&M costs increase in the year 2002 because of an assumed switch from crystalline silicone to thin film technology. 



\section{Biofuels Supply Submodule}

\section{Model Purpose}

The objective of the Biofuels Supply Submodule (BSS) is to provide the NEMS Petroleum Market Module (PMM) with supply curves for corn-derived ethanol, thus allowing the PMM to forecast transportation ethanol demand through the year 2010. A secondary objective is to report the energy content of ethanol produced for transportation fuel.

To be consistent with the market clearing mechanism adopted for NEMS, the BSS provides ethanol prices in the form of annual price-quantity curves. The curves, derived from an ethanol production cost function, represent the prices of ethanol at which associated quantities of. transportation ethanol are expected to be available to refineries for blending with gasoline.

\section{Relationship of the Biofuels Submodule to Other Models}

The BSS's major NEMS linkages are with the Petroleum Market Module and the Coal Market Module (CMM). There is a two-way exchange of information between the BSS and PMM: the PMM provides the BSS with regional diesel fuel prices, while the BSS provides the PMM with delivered ethanol prices. The CMM serves as a source of energy price information for determining the total cost of converting corn into ethanol.

The delivered ethanol prices are provided to the PMM in the form of two supply curves, one for the East North Central Census Division (NEMS region 3), and one for the West North Central Census Division (NEMS region 4). ${ }^{8}$ These two Census divisions constitute the major ethanol producing regions in the United States, and are the only two Census divisions considered for the

$\therefore$ AEO94 ethanol production forecasts.

To determine the delivered ethanol price, the contribution of the net cost of corn feedstock production must be factored in to the total unit price of ethanol. Diesel fuel prices, in dollars per gallon, are also considered as one of two energy cost variable inputs to the ethanol cost projected by the BSS. The other energy price input to the BSS's ethanol production cost function is the price of energy for corn feedstock processing and ethanol conversion. Coal prices are used as a proxy for industrial energy costs. Regional forecasts of energy prices (dollars per million Btu) to industrial consumers are supplied by the CMM.

\footnotetext{
${ }^{8}$ All regional data inputs to the BSS ethanol prodnction cost function are by Petroleum Administration for Defense Districts (PADDs). The calculated ethanol prices and quantities are mapped to the two Census divisions prior to being written to the NEMS price/quantity COMMON blocks.
} 
Inputs from other NEMS modules are summarized as follows:

- Regional delivered price of diesel fuel to the agricultural/transportation sector. This is obtained from the Petroleum Market Module, and is used for computation of com feedstock prices.

- Regional delivered price of process energy to industrial consumers, obtained from the Coal Market Module, are used to compute the conversion costs in the regional ethanol supply curves.

- Yield on AA utility bonds. This is obtained from the Macroeconomic Activity Module, and is used for calculating the capital cost factor. (See Appendix 5-B, "Mathematical Description,". for the derivation of the capital cost factor.)

A major source of data supplied to the BSS comes from runs of a model external to the NEMS environment. This model, the Agricultural Resources Interregional Modeling System (ARIMS), was the source of the corn feedstock cost-supply relationships used in the BSS's ethanol cost function. ARIMS was developed at the U.S. Department of Agriculture in the 1980's. The ARIMS is a linear programming. resource allocation model that was restructured to account for the value of the by-products produced in the corn-to-ethanol conversion process and to project the net cost of corn feedstocks. ${ }^{9}$ In other words, the projected by-product values were credited against the price" of corn. The variability of the-market price for the feedstock corn and the conversion by-products and the variable influences of competitive uses for corn (e.g., for producing corn syrup) gives rise to broad fluctuations in net corn feedstock prices. All of these factors are considered in the ARIMS model.

ARIMS was run for 1995,2005 , and 2015 to provide price-quantity data for ethanol feedstocks. The changes in the competitive agricultural infrastructure modeled by ARIMS typically occur so slowly that the three years of model projections were deemed sufficient to bracket the behavior within the forecast horizon. ${ }^{10}$ Interpolation was used to derive data points for the remaining AEO94 forecast years. ARIMS is not integrated with NEMS, so that sensitivity analysis between NEMS and ARIMS is not currently feasible.

\footnotetext{
'The net contribution of the cost of corn feedstocks to the price of ethanol is reduced over time by gradually improving conversion process yields. it is also affected by variations in the energy costs for prodincing com. PDIESEL, the price of diesel fuel, was the proxy variable used to model the sensitivity of corn production costs to variable energy costs. Analyses were performed off-site and summary statistics are not currently available.

${ }^{10}$ Energy Information Administration, "Component Design Report for Biofuels (Ethanol) Supply Submodnle Renewable Fuels Model - National Energy .Modeling System, Draft 7/2/92.
} 


\section{Modeling Rationale}

\section{Theoretical Approach}

The BSS uses a process costing approach to model the impacts of net feedstock production costs plus the capital, operating, and process energy costs associated with converting the corn feedstocks to ethanol. In other words, each of the above factors contributes a part of the total price of ethanol projected by the BSS.

As mentioned above, the ARIMS supplied the data for the feedstock cost function variables. Since ethanol feedstock supply curves are a function of many factors (i.e., time, geographic location, demands for traditional agricultural commodities (domestic and foreign, crop and livestock), agricultural production technology, and land availability), the BSS needed the capability to relate such factors in a summary fashion to feedstock resource requirements under competitive agricultural market conditions. The ARIMS provides that capability with the use of a general equilibrium modeling framework.

The ARIMS was used to project corn crop demand and production resources and technology. Subject to constraints that were intended to capture the most.important attributes of the agricultural market, the ARIMS model minimized the net cost of producing the specified quantities of corn produced as feedstock for ethanol, and the use of the feed by-products. The crop feedstock demand for ethanol production was set at various levels; with all other aspects of the model held constant. This allowed the linear program to develop sets of points that were used to estimate the step function feedstock supply curve.

Note that with this theoretical approach, only the agricultural, or feedstock production costs are modeled as a function of the total quantity of ethanol produced. The conversion plant process costs, (capital, operating, and process energy) are modeled as process cost which are independent of production quantities. The feedstock production cost components are estimated statistically, whereas the conversion process costs are determined from engineering concepts and data. Actual ethanol conversion process data are, for the most part, proprietary.

\section{Fundamental Assumptions .}

\section{Ethanol Production Capacity}

An important modeling consideration is the imposition of a constraint on the amount of ethanol production capacity that can be added in any one year. Such a constraint would theoretically prevent unrealistically large increases in production capacity from occurring suddenly in response to potential structural market changes. On the other hand, our research determined that such capital expansion considerations are unnecessary for this modeling application because the lead time for capital expansion is very -short and because the feedstock availability represents the major constraint to the expansion of ethanol production facilities. 
For the AEO94, no structural changes to feedstock markets are assumed to occur during the forecast horizon. It is assumed that production capacity is utilized fully to meet refinery ethanol demand, and that there is sufficient ethanol production to meet refinery ethanol demand requirements.

\section{Ethanol Production Costs}

The ethanol supply-price curve reflects offsetting influences stemming from the effects of increased corn production and improvements in corn-to-ethanol conversion technologies. Net feedstock prices are projected to increase as production. increases due to two primary reasons. First; land becomes scarcer, causing both land and feedstock costs to increase, and second, feed by-products become less valuable as larger feedstock quantities are produced. Over time, however, the technologies for growing corn and converting it to ethanol are projected to improve, resulting in downward pressure on ethanol production prices. The BSS models the net effect of all of these factors.

In addition to feedstock prices and quantities derived from ARIMS, the BSS requires feedstock conversion and energy cost data. The conversion cost data were derived from the U.S. Department of Agriculture Report 585 Ethanol: Economic and Policy.Tradeoffs, and the analytical judgment of Dr. Anthony Turhollow. These costs were developed for the two Census Divisions (3 and 4) that comprise PADD region 2. Although the BSS has the ability to include ethanol production subsidies, they were set at zero for AEO94. The ethanol blender's excise tax credit, which is currently $\$ 0.054$ per gallon of gasohol (10 percent ethanol, 90 percent gasoline), is modeled in the PMM.

Quantities of energy needed to convert corn to ethanol are assumed to be a positive linear function of input values for years 1,16 , and 26 , and to remain constant, at the year 26 value, for years 27,28 , and 29. (The AEO94 runis utilized cost data only up to year 20). Current facilities use 50,000 Btu per gallon of ethanol produced; while state-of-the-art plants run as low as 40,000 Btu per gallon. These two values are used as input values for years 1 and 16, respectively, with later years based on a linear trend of the first two values. This linear interpolation procedure was based on the assumption that, over time, ethanol facilities have become more energy efficient, and will continue to do so as they convert corn to ethanol at higher conversion rates and adopt technology improvements such as organisms with higher tolerances for sugars and ethanol, and molecular sieves to separate water from products. The feedstock conversion energy prices used to develop the feedstock cost function are national prices. Regional prices were not necessary since the relationship between feedstock production costs and energy prices is thought to be relatively constant across regions.

Operating costs for feedstock conversion are also assumed to be a positive linear function of input values for years 1 and 16, but remain constant at the year 16 value for the remaining forecast years. The first-year 1990 value of $\$ 0.30$ per gallon is an average plant cost for 1987 , .while the year 16 value of $\$ 0.27$ per gallon is a projected state-of-the-art plant cost. 


\section{Treatment of Energy Crop. Ethanol Feedstocks}

Significant production of energy crops (e.g., grasses and short rotation trees) for ethanol production is not expected until about 2005. The conversion technology is at a stage wherein demonstration facilities for this technology are not expected to be operational until 1999, at the earliest. A few years of operating experience with the demonstration facilities will be required, and constructing the conversion facilities will also require several years time. Therefore, developing their supply functions for inclusion in the BSS will be deferred until a later AEO.

\section{Alternative Approaches}

Prior to the BSS, the EIA had no in-house modeling system for forecasting alcohol fuel production and demand. The ethanol forecasts for previous $A E O$ reports were consensus forecasts prepared by Oak Ridge National Laboratory (ORNL); based on the inter-laboratory renewable fuels energy white paper prepared in 1990. Subsequent to the AEO92, a prototype modeling system, utilizing other existing models and a simple supply representation for the production of ethanol, was developed by ORNL. The prototype model consisted of a supply component, a demand component, and a market-ciearing process."

For the demand component, an existing model, the Alternative Motor Fuel Use Model (AMFU). was adopted. AMFU is a model used to forecast fuel usage, vehicle usage, and vehicle stock for up to a 40-year horizon. It has the characteristics of both an accounting model for vehicle stocks, and, an econometric model with economic activity and prices of fuels for forecasting total fuel demand. The fuel use portion of AMFU assumes that vehicle usage is a function of fuel cost and economic growth, as estimated by statistical models. The proportion of vehicles using any particular fuel (i.e., gasoline, diesel fuel, ethanol) is represented by an algebraic system that includes the relative prices of alternative fuels.

The supply component of the prototype model was represented by a step-function supply curve. The energy crops alternative was represented as a flat supply curve. The sources of these supply functions were Abt Associates (1991) and Tyson (1990), respectively.'

Finally, a market-clearing process was used to find an equilibrium solution. The demand model was run for the lowest available price (as determined by the supply curve) of ethanol. If the demand for ethanol exceeded the available supply at that price, the next step of the supply curve was tried. When demand met the available supply, the solution was complete.

Unlike the prototype, the BSS analyzes supply factors only. Market penetration of alternativefueled vehicles will be determined in the Transportation Demand Module, and the quantities of ethanol blended with gasoline will be determined by the Petroleum Market Module. No

\footnotetext{
'11Lee, R., S.M. Cohn, and R.D. Perlack. 1991. Prototype of an Integrated Model for Projecting Biofuels Consumption. Draft report prepared for Energy Information Administration, U.S. Departinent of Energy. Oak Ridge National Laboratory, Oak Ridge, TN.
} 
quantitative models for forecasting the production or consumption of ethanol have been identified for application in the BSS.

\section{Biofuels Submodule Structure}

\section{Submodùle Flow Diagram}

A flow diagram showing the main computational steps and relationships of the Biofuels Supply Submodule is shown in Figure 4.

Figure 4. .Biofuels Supply Submodule Flowchart

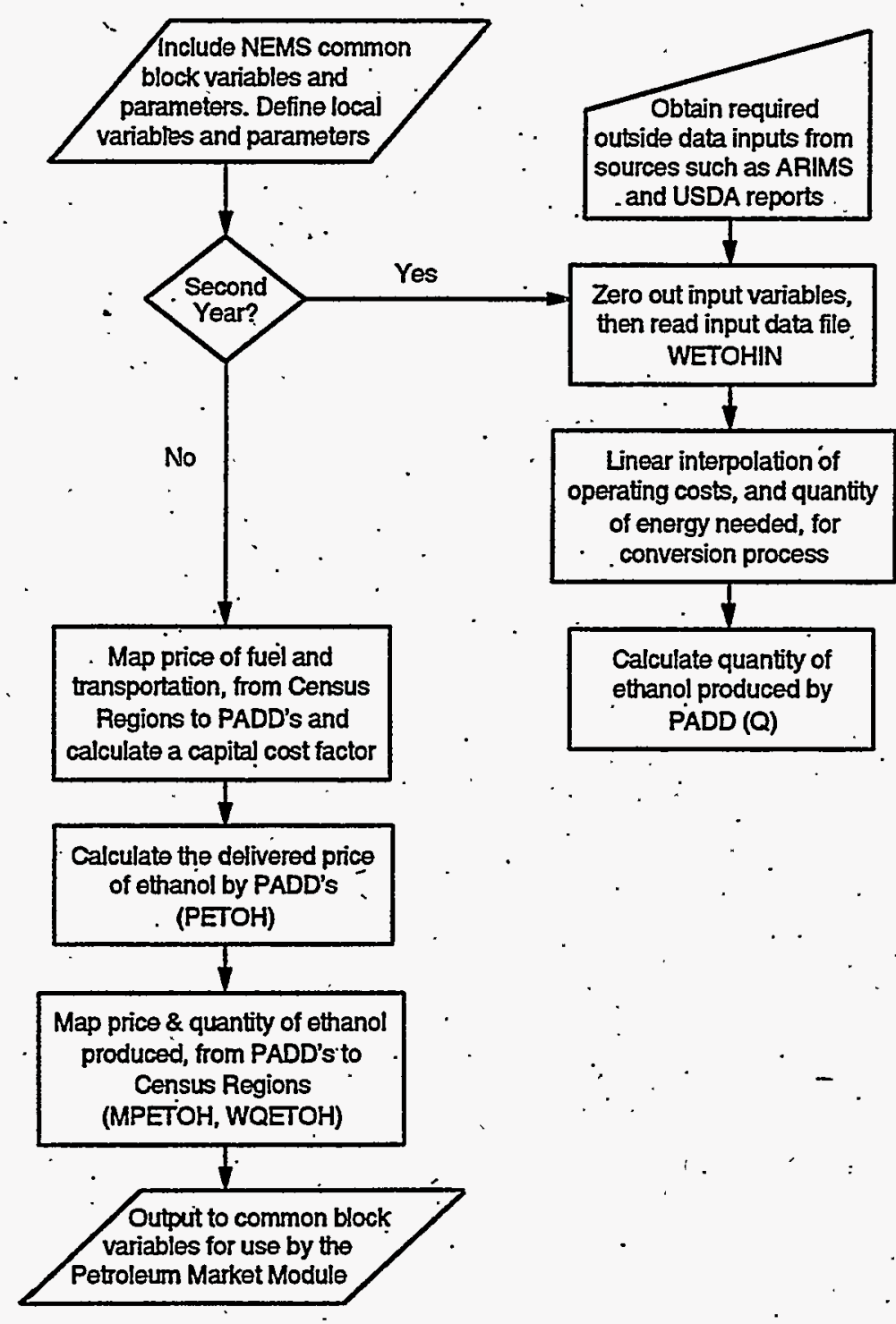




\section{Key Computations and Equations}

The main computations performed by the BSS involve the derivation of a single ethanol supplyprice curve. The computations consist of three major steps:

1. Reading in ethanol supply and component cost data, and performing annual interpolations of data values provided on a multi-year basis,

2. Computation of ethanol supply curve (price/quantity) coordinates.

3. Derivation of delivered ethanol prices, calculated as a function of the supply curve coordinates from step 2 . .

Each of these steps is described below.

After reading in the single input data file, (WETOHIN), the BSS performs a simple linear interpolation on two of the input data variables. These two variables, indexed in Table 5A-1, are OPCST (operating cost for feedstock conversion technologies, exclusive of energy) and $Q E N$ (quantity of energy needed for feedstock conversion). The BSS gets data values for these variables for 3 years, corresponding to years 1,16 , and 26. Linear interpolations are performed to calculate intermediate yearly values.

The next step involves the calculation of feedstock costs as a function of quantity and year. Readers should recognize this as a standard interpolation routine (Equation 5-1) supplied to the data in Table 3. The input data file supplies historical data on costs, as well as ARIMS forecasts, at selected quantities of ethanol production. ${ }^{12}$ Because significant ethanol production is currently limited to PADD 2 (Census divisions 3 and 4), the BSS calculates ethanol supply quantities and prices only for Petroleum Administration.for Defense District (PADD) 2; supply quantities and prices for the other four PADDs are fixed at zero. The input file therefore supplies the skeleton, for selected years, of the corn production costs $\operatorname{COST}_{p, t e}$ at diesel price $p$, year $t$ and production volume $e$. The input file also supplies the diesel price vector $D_{p}$ and the vector $Q_{e}$ that contains the quantities for each of the volume steps. Table 3 shows a the skeleton matrix used for AEO94. The BSS interpolates values for the $\operatorname{COST}_{p, t_{e}}$ matrix for years $t$ not given in the input file, and fills in the same cost at all diesel price points in the historical years.

After the skeleton matrix $\operatorname{COST} T_{p, \dot{c} \boldsymbol{e}}$ has been filled in for all years, a supply curve for a given diesel price $P D I E S E L_{p, r}^{-}$is interpolated from the matrix using the formula:

${ }^{12}$ All ethanol produced is assumed to be delivered to refineries. . 
Table 3. Corn Production Cost Skeleton Matrix (\$/gal)

\begin{tabular}{|c|c|c|c|c|c|c|c|}
\hline \multicolumn{2}{|c|}{18} & \multicolumn{5}{|c|}{ 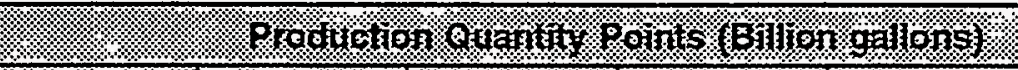 } & \multirow{2}{*}{ 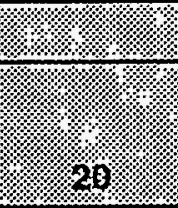 } \\
\hline (: & PIoser & $\sqrt{7}=$ & (4: & 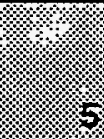 & U: & (i) & \\
\hline 1995 & 6 & 0.214 & 0.315 & 0.346 & & & \\
\hline 1995 & 8 & 0.233 & 0.336 & 0.372 & & & \\
\hline 1995 & 10 & 0.250 & 0.359 & 0.402 & & & \\
\hline 1995 & 12 & 0.272 & 0.378 & 0.425 & & & \\
\hline 1995 & 14 & 0.279 & 0.408 & 0.454 & & & \\
\hline 2005 & 6 & 0.266 & 0.301 & 0.331 & 0.371 & 0.420 & \\
\hline 2005 & 8 & 0.279 & 0.327 & 0.347 & 0.383 & 0.435 & \\
\hline 2005 & 10 & 0.306 & 0.379 & 0.379 & 0.407 & 0.466 & \\
\hline 2005 & 12 & $0.333^{\circ}$ & 0.394 & 0.412 & 0.431 & 0.488 & \\
\hline 2005 & 14 & 0.351 & 0.415 & 0.436 & 0.470 & 0.514 & \\
\hline 2015 & 6 & 0.255 & 0.281 & 0.300 & 0.326 & 0.351 & 0.442 \\
\hline 2015 & 8 & 0.281 & 0.296 & 0.316 & 0.342 & 0.367 & 0.465 \\
\hline 2015 & 10 & 0.307 & 0.327 & 0.349 & 0.374 & 0.400 & 0.511 \\
\hline 2015 & 12 & 0.333 & 0.360 & 0.382 & $0.400^{\circ}$ & 0.443 & 0.551 \\
\hline 2015 & 14 & 0.350 & 0.383 & 0.403 & 0.423 & 0.461 & 0.598 \\
\hline
\end{tabular}

where:
$F C_{p r, i e} \cdot=$. Cost of producing corn in PADD pr=2 in year $t$ for volume step $e$ (\$/gal),
$\operatorname{COST}_{p, 5 e}=$ Production cost matrix by diesel price step $p$ in year $t$ for volume step $e$ (\$/gal),
PDIESEL $_{p r, t}=$ Price of diesel oil in PADD $p r=2$ in year $t$ (\$/MMBtu), and
$D_{p} \quad . \quad=\quad$ Diesel oil price step quantity for each step $p$ (\$/MMBtu),

with

$$
D_{p-T}<P D I E S E L_{p r, t}<D_{p}
$$


Indices

$$
\begin{aligned}
& \boldsymbol{e} . \quad=\quad \text { point on the supply curve, volume step } 1 \text { to } 5 \\
& f \quad=\quad \text { fuel(units in parentheses); 1=gasoline(gallons), } 2=\text { diesel(gallons), } \\
& \text { 3=LPG(gallons), 4=natural gas(MMBtu), 5=electricity(Kwh), } \\
& \text { 6=coal(MMBtu), } 7=\text { fuel for energy crop conversion (MMBtu). } \\
& i \quad=\quad \text { crop; 1=corn, 2=energy crops } \\
& s r \quad=\quad \text { Census Region, } s r=1 \text { to } 9 \\
& \text { pr } \quad=\text { PADD, } p r=1 \text { to } 5 \\
& t \quad=\quad \text { year, } 1990 \leq t \leq 2015
\end{aligned}
$$

The third major computational step involves the derivation of delivered ethanol prices for each PADD. The ethanol prices, PETOH, are calculated as a linear function of (1) the corn feedstock cost $F C_{p r, t, e}$ shown above, (2) the price of diesel fuel, which serves as a proxy for all of the energy costs of producing the feedstock and transporting it to the conversion facility, and (3) corn-to-ethanol conversion facility process cost contributions, namely, capital, non-energy-related operating costs, and process energy costs.

The delivered ethanol price equation is as follows:

$$
\begin{aligned}
& P E T O H_{i, p r, t, e}=\dot{F} C_{p r, j e}+C A P C S T_{i, t} * C C F+O P C S T_{i, t} \\
& +Q E N_{i, t} * P E N_{p r, t}-S U B
\end{aligned}
$$

where:

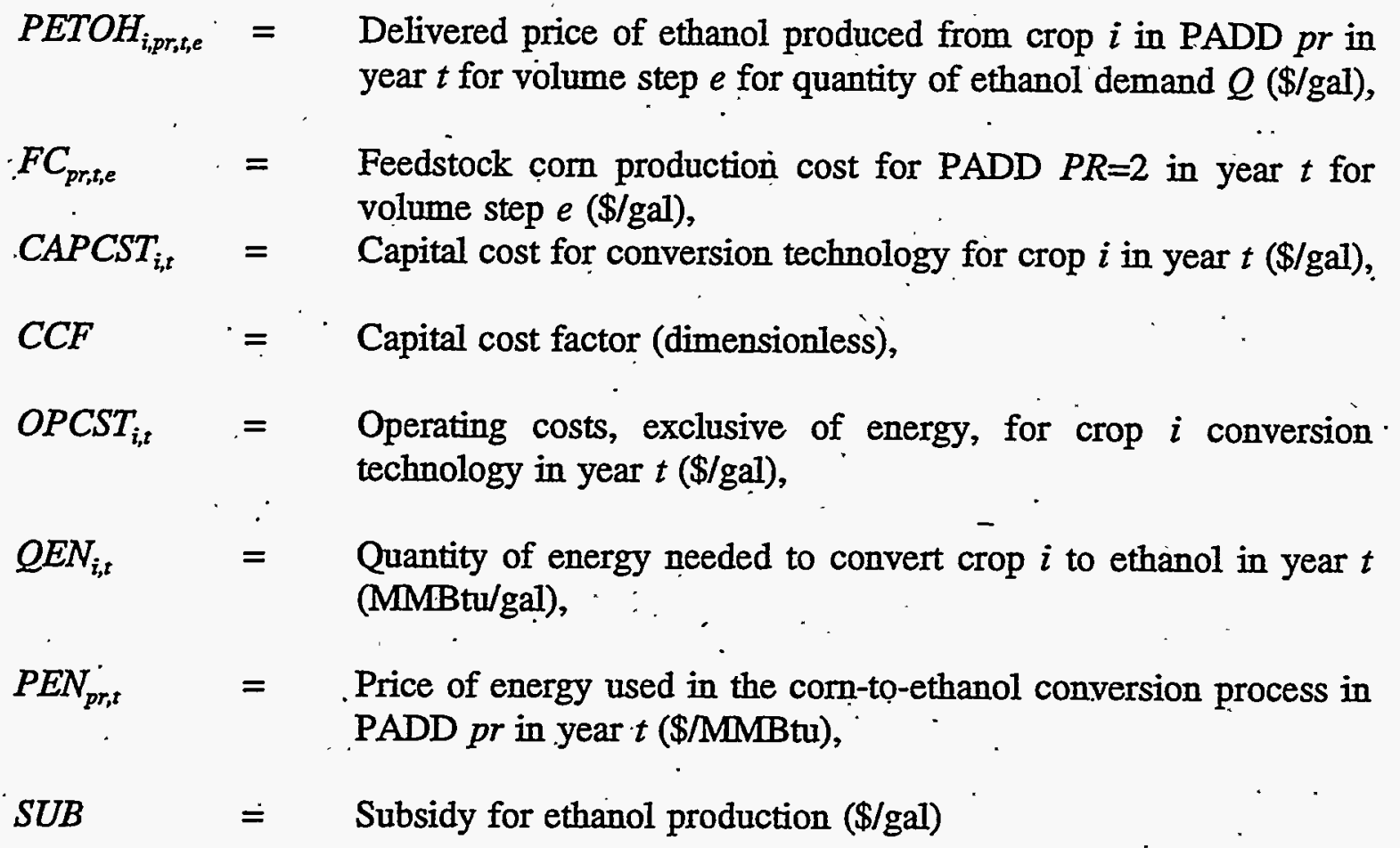





\section{Appendix 5-A: Inventory of Variables, Data, and Parameters}

This Appendix describes the variables, data inputs, and parameter estimates associated with the Biofuels Submodule. Table 5A-1 provides a tabular listing of model input data and input variable parameters. The table contains columns with information on item definitions, modeling dimensions, data sources, measurement units, and documentation page references. Similarly, Table 5A-2 provides an indexed listing of model output data and parameters.

The remainder of Appendix 5-A consists of detailed descriptions of data inputs and variables, including discussions on supporting data assumptions and transformations.

Table 5A-1. NEMS Biofuels (Ethanol). Supply Submodule Inputs

\begin{tabular}{|c|c|c|c|c|}
\hline Yrooffor: & (1.1. \% & 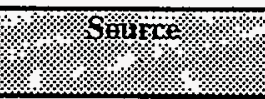 & (1) & Gage Referenes. \\
\hline CAPCST & $\begin{array}{l}\text { Capital cost for conversion technology } \\
\text { for crop } i \text { in year } t\end{array}$ & $\begin{array}{l}\text { USDA/ERS. } 1988 . \\
\text { Report \#585 }\end{array}$ & \$/gallon & $136,142,145,146$ \\
\hline $\cos T$ & $\begin{array}{l}\text { Cost of producing corn in PADD } 2 \text { at } \\
\text { diesel price step } P \text { in year } t \text { at volume } \\
\text { step } e\end{array}$ & $\begin{array}{l}\text { ARIMS Output } \\
\text { Trumble. } 1994\end{array}$ & \$/gallon & $135,136,142,145$ \\
\hline$D$ & Values of diesel price steps $p$ & Trumble. 1994 & S/MMBtu & 137 \\
\hline$Q$ & Quantity at each volume step $e$ & Trumble. 1994 & billion gallons & 137 \\
\hline$M C \_R M P U A A N S$ & Yield on AA utility bonds for year $t$ & $\begin{array}{l}\text { Macroeconomic } \\
\text { Market Module }\end{array}$ & Dimensionless & 137 \\
\hline OPCST & $\begin{array}{l}\text { Operating costs, exclusive of energy, } \\
\text { for.conversion technology of crop } i \text { in } \\
\text { year } t\end{array}$ & $\begin{array}{l}\text { USDA/ERS. } 1988 . \\
\text { Report } 585\end{array}$ & \$/gallon & $133,138,141$ \\
\hline PADD2CR & $\begin{array}{l}\text { Conversion rates to convert from PADD } \\
p r \text { to Census Region } s r\end{array}$ & A. Turhollow & Dimensionless & 138 \\
\hline PDSTR & $\begin{array}{l}\text { Price of diesel for transportation in } \\
\text { Census Region } s \text { in year } t\end{array}$ & $\begin{array}{l}\text { Petroleum Market } \\
\text { Module }\end{array}$ & \$/gallon & 136,138 \\
\hline PCLIN & $\begin{array}{l}\text { Price of coal for industrial use in } \\
\text { Census Region } s r \text { in year.t }\end{array}$ & $\begin{array}{l}\text { Coal Market } \\
\text { Module }\end{array}$ & \$/MMBtu & 136,138 \\
\hline QEN & $\begin{array}{l}\text { Quantity of energy needed to convert } \\
\text { crop } i \text { to ethanol in year } t\end{array}$ & $\begin{array}{c}\text { Marland \& } \\
\text { Turhollow. } 1991\end{array}$ & MMBtu/galion & $137,144,146$ \\
\hline QFUEL & $\begin{array}{l}\text { Quantity of fuel type } f \text { used in the } \\
\text { production of crop } i \text { in year } t\end{array}$ & $\begin{array}{l}\text { Marland \& } \\
\text { Turhollow. } 1991\end{array}$ & $\begin{array}{l}\text { Gallons for } f=1,2,3 \\
\text { MMBtu for } f=4 \\
k W h \text { for } f=5\end{array}$ & 139 \\
\hline
\end{tabular}


Table 5A-2. NEMS Biofuels (Ethanol) Supply Submodule Outputs

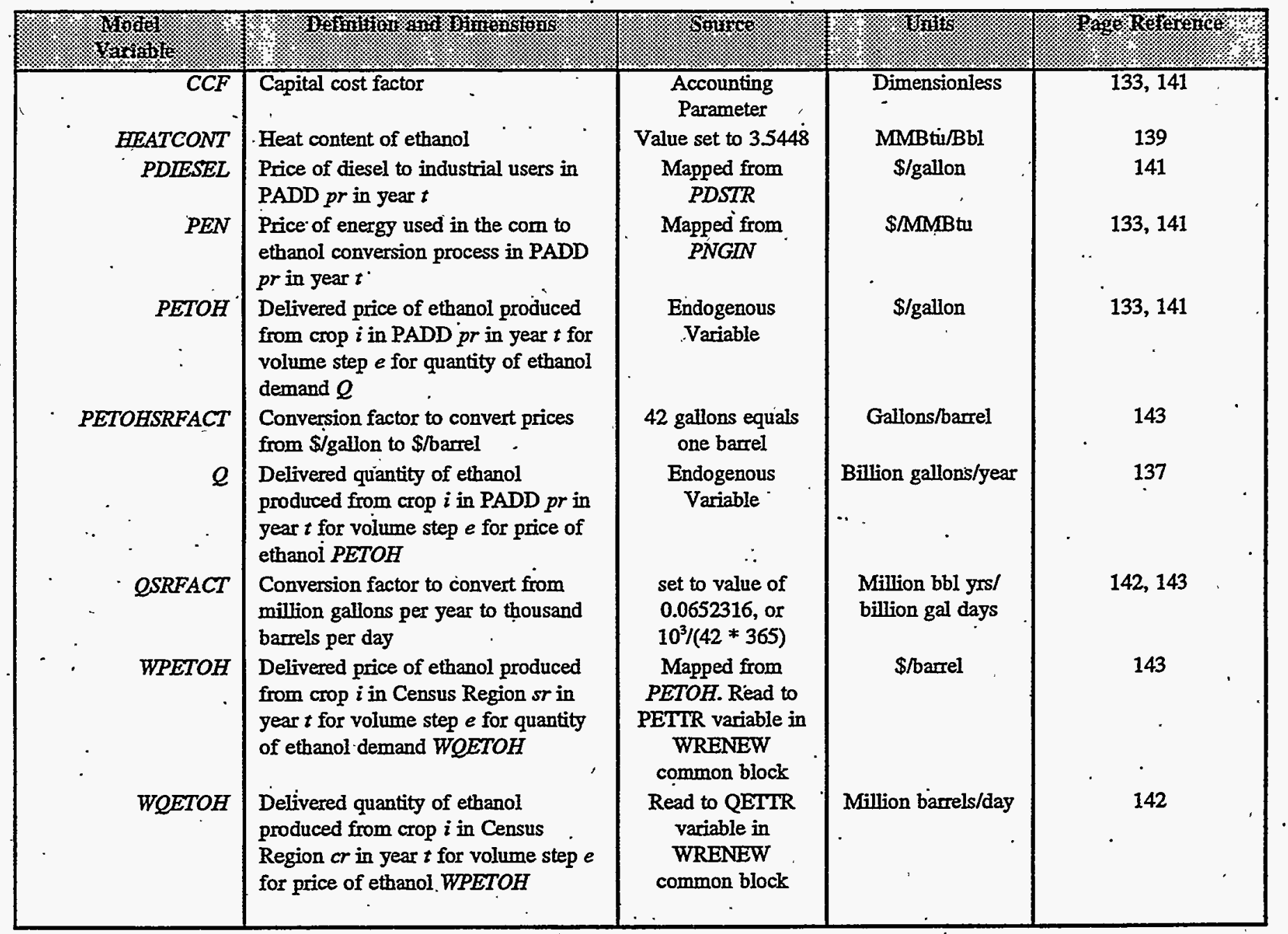

\section{MODEL INPUT: $\quad$ CAPCST}

DEFINITION: - Capital cost for conversion technology for crop $i$ in year $t$.

Given only for corn since the BSS is currently concerned only with corn as a feedstock. The current value is $\$ 2.00$ per gallon, and is the same for all years. Located in the WETOHIN input data file.

SOURCE:- USDA/ERS. 1988: Ethanol: Economic and Policy Tradeoffs. Agricultural Economic Report No. 585. Resources and Technology Division, Economic Research Service, U.S. Department of Agriculture, Washington, D.C. 


\section{MODEL INPUT: $\quad$ COST}

DEFINITION: - Outputs from the ARIMS model from cases executed at each of the price steps $p$ and each of the quantity steps $e$.

Values represent the cost of producing the com necessary to produce $Q_{e}$ billion gallons of ethanol if the price were $D_{p}$ in year $t$.

Values are given only for PADD 2. Quantities of ethanol produced outside of PADD 2 are currently insignificant, so all production from ethanol is shown in PADD 2. Located in the WETOHIN input data file.

SOURCE: ARIMS model outputs. David A. Trumble. 1994. Estimation of supply Curve for Ethanol with Corn as the Feedstock. Oak Ridge National Laboratory.

\section{MODEL INPUT: $\quad D$}

DEFINITION: Diesel oil price steps $p$.

The diesel oil prices for which the ARMMS model was executed in each year. The BSS assumes. that $\operatorname{COST} T_{p, t e}$ was generated from a matrix of ARIMS cases for each $D_{p}$ and $Q_{e}$.

SOURCE: ARIMS model inputs. David A. Trumble. 1994. Estimation of supply Curve for Ethanol with Corn as the Feedstock. Oak Ridge National Laboratory.

MODEL INPUT: $Q$

DEFINITION: Volume price steps $e$.

The ethanol volume steps for which the ARIMS model was executed in each year. The BSS assumes that $\operatorname{COS} T_{p, z e}$ was generated from a matrix of ARIMS cases for each $D_{p}$ and $Q_{e}$.

SOURCE: ARIMS model inputs. David A. Trumble. 1994. Estimation of supply Curve for Ethanol with Corn as the Feedstock. Oak Ridge National Laboratory.

\section{MODEL INPUT: $\quad$ MC_RMPUAANS}

DEFINITION: Yield on AA utility bonds for year $t$.

Located in the Macroeconomic common block, MACOUT.

SOURCE: Generated by the Macroeconomic Activity Module. 
MODEL INPUT: $\quad$ OPCST.

DEFINITION: Operating costs, exclusive of 'energy, for conversion technology of crop $i$ in year $t$.

Given only for corn since the BSS is currently modeling only corn-derived ethanol. Values are: $\$ 0.30 /$ gal. for $1990, \$ 0.27 / \mathrm{gal}$. for 2005 . $\$ 0.27 / \mathrm{gal}$. for 2015 . Located in the WETOHIN input data file.

SOURCE: USDA/ERS. 1988. Ethanol: Economic and Policy Tradeoffs. Agricultural Economic Report No. 585. Resources and Technology Division, Economic Research Service, U.S. Department of Agriculture, Washington, D.C.

\section{MODEL INPUT: $\quad P A D D 2 C R$}

DEFINITION: $\quad$ Conversion rates to convert from PADD $p r$ to Census Region $s r$.

Values are given for each PADD and Census Region. Most PADD's map one-to-one to a Census Region. Only PADD 2 maps into two different Census Regions. Located in the WETOHIN input data file.

SOURCE: Generated by Dr. Anthony Turhollow, Oak Ridge National Laboratory, based on historical ethanol production from corn feedstocks.

\section{MODEL INPUT: $\quad$ PDSTR}

DEFINITION: $\quad$ Price of diesel for transportation in Census Region $s r$ in year $t$.

Located in the NEMS Price common block (MPBLK).

SOURCE: Generated by the Petroleum Market Module.

- MODEL INPUT: $\quad$ PCLIN

DEFINITION: $\quad$ Price of coal for.industrial use in Census Region $s r$ in year $t$.

Located in the Price common block, (MPBLK).

SOURCE: Generated by the Coal Market Module. 
MODEL INPUT: $\quad$ QEN

DEFINITION: : Quantity of energy needed to convert crop $i$ to ethanol in year $t$.

Given only for corn since the BSS is currently concerned only with corn as a feedstock. Values, in million Btu per gallon, are as follows: 0.050 in 1990, 0.040 in 2005, 0.035 in 2015. This decreasing trend is based on the assumption that energy required decreases linearly over time. Located in the WETOHIN input data file.

SOURCE: Marland, G. and A.F. Turhollow. 1991. " $\mathrm{CO}_{2}$ Emissions From the Production and Combustion of Fuel Ethanol from Corn." Energy, 16(11/12):1307-1316.

\section{MODEL INPUT: $\quad$ QFUEL}

DEFINITION: Quantity of fuel type $f$ used in the production of crop $i$ in year $t$.

Given only for corn since the BSS is currently concerned only with corn as a feedstock. Values cover seven different fuel types and 20 forecasts, and remain constant for the duration of the forecast horizon. The values for fiel type six, coal, are all zero. Fuel type seven is reserved for fuel provided for the feedstock conversion process. Located in the WETOHIN input data file.

SOURCE: Marland, G. and A.F. Turhollow. 1991. " $\mathrm{CO}_{2}$ Emissions From the Production and Combustion of Fuel Ethanol from Corn." Energy, 16(11/12):1307-1316.

\section{MODEL INPUT: HEATCONT}

DEFINITION: Heat content of ethanol in transportation fuel, high-heating value:

SOURCE: Marland, G. and A.F. Turhollow. 1991. "CO ${ }^{\natural}$ Emissions From the Production and Combustion of Fuel Ethanol from Corn." Energy, 16(11/12):1307-1316. 


\section{Appendix 5-B: Mathematical Description}

This Appendix provides the detailed mathematical specification of the Biofuels (Ethanol) Supply Submodule as presented in the RFM FORTRAN code execution sequence. Subscript definitions are also as they appear in the FORTRAN code.

The ARIMS model is executed for a series of cases defined at a series of ethanol production quantities $Q_{e}$ and diesel fuel prices $D_{p}$ and years $t$, to create a matrix of corn production cost solutions $\operatorname{COST}_{p, t e}$ in $\$$ /gal. These are input to the BSS, and interpolated over years.

For a given NEMS diesel price PDIESEL $L_{p r, p}$, where the PADD $p r=2$, the BSS interpolates a corn cost curve $F C_{p r, t, e}$ for PADD 2 that gives the cost in that year to produce each of the ethanol quantities $Q_{e}$ at that diesel price. Here, PDIESEL $L_{p, r}$ represents the NEMS price of diesel fuel PDSTR, in \$/MMBtu.

The delivered price of ethanol is calculated with the following equation:

$$
\begin{aligned}
& \text { PETOH }_{1, p r, t, e}=F_{\text {pr, }, e}+C A P C S T_{1, t} * C C F \\
& +O P C S T_{1, t}+Q E N_{1, t} * P E N_{p, t}
\end{aligned}
$$

where:

$$
\begin{aligned}
& \text { PETOH }_{i, p r, i, e}=\text { Delivered price of ethanol produced from crop } i \text { in PADD } p r \text { in } \\
& { }^{9} \$ \text { year/gal }{ }^{2} \text { ) to } \mathrm{B} 1 \text {. } \\
& P D I E S E L_{p s, t}=\text { price of diesel for PADD pr.in year } t(\$ / M M B t u) \\
& \text { CAPCST }_{i, \varepsilon}=\quad \text { capital cost for conversion technology for crop } i \text { in year } t \text { (\$/gal) } \\
& \text { CCF }=\text { Capital cost factor (dimensionless) } \\
& \operatorname{OPCST}_{i, r}=\text { operating costs, exclusive of energy, for crop } i \text { conversion } \\
& \text { technology in year } t \text { (\$/gal) } \\
& Q E N_{i, t} \quad=\quad \text { quantity of energy needed to convert crop } i \text { to ethanol in year } t \\
& \text { (MMBtu/gal) } \\
& P E N_{p r, t} \cdot=\text { price of energy used in the corn-to-ethanol conversion process in } \\
& \text { PADD } p r \text { in year } t(\$ / M M B t u)
\end{aligned}
$$


Indices

$$
\begin{aligned}
& e \quad=\quad \text { point on the supply curve, volume step } 1 \text { to } 5 \\
& f \quad=\quad \text { fuel(units in parentheses); } 1 \text { =gasoline(gallons); } 2=\text { diesel(gallons), } \\
& 3=\mathrm{LPG} \text { (gallons), 4=natural gas(MMBtu), 5=electricity(Kwh), } \\
& 6=\text { coal(MMBtu), } 7=\text { fuel for energy crop conversion (MMBtu). } \\
& i \quad=\quad \text { crop; } 1=\text { corn, } 2=\text { energy crops } \\
& s r \quad=\quad \text { Census Region, } s r=1 \text { to } 9 \\
& \text {.pr } \quad=\quad \text { PADD, } p r=1 \text { to } 5 \\
& t=\text { year, } .1990 \leq t \leq 2015
\end{aligned}
$$

The capital cost factor (CCF) used in equation 5B-2, which is based on a 30-year amortization. period, is calculated as follows:

$$
C F F=M C \_R M P U A A N S_{t} *\left(1+M C_{-} R M P U A A N S_{\ell}\right)^{30} /\left(\left(1+M C \_R M P U A A N S_{t}\right)^{30}-1\right)
$$

where:

$$
\begin{aligned}
& \text { MC_RMPUAANS }=\quad \begin{array}{l}
\text { yield on AA-grade utility bonds (a Macroeconomic Activity } \\
\text { Module output variable) }
\end{array}
\end{aligned}
$$

The quantity of ethanol used as a transportation fuel, $\mathrm{WQETOH}_{\mathrm{cr}, \mathrm{t},}$, is derived from the following equation:

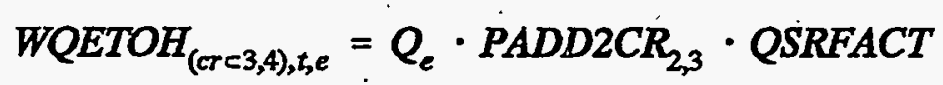

where:

$$
\begin{aligned}
& \text { WQETOH }=\quad \begin{array}{l}
\text { Quantity of ethanal used as a transportation fuel, in barrels per day, } \\
\text { for Census division } 3 \text { and } 4, \text { year } t \text {, and supply step } e,
\end{array} \\
& \begin{array}{l}
\text { quantity of ethanol produced from crop } i \text { in PADD } 2 \text { in year } t \text { for } \\
\text { volume step } e \text { (billion gallons/year), }
\end{array} \\
& P A D D 2 C R_{2,3}=\quad \begin{array}{l}
\text { Conversion factors to convert from PADD } 2 \text { to Census Regions } 3 \\
\text { and } 4,
\end{array} \\
& Q S R F A C T=\begin{array}{l}
\text { Conversion factor to convert from million gallons per year to } \\
\text { thousand barrels per day: }
\end{array}
\end{aligned}
$$

The price of ethanol used as a transportation fuel, $\mathrm{WPETOH}_{c \mathrm{r}, \mathrm{e}}$, is derived from the following equation: 


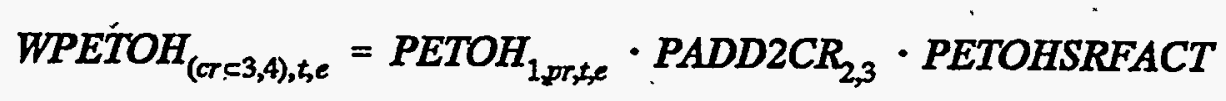

where:

WPETOH

$\mathrm{PETOH}_{e}$

$P A D D 2 C R_{2,3}$

PETOHSRFACT . : $=\quad$ Price of ethanol used as a transportation fuel, in $\$$ per - barrel, for Census division 3 and 4 ; year $t$, and supply step $e$,

$=\quad$ Price of ethanol produced from corn in PADD 2 in year $t$ for volume step $e$ (\$/gallon),

$=$ Conversion factors to convert from PADD 2 to Census Regions 3 and 4,

Conversion factor to convert from gallons to barrels. 


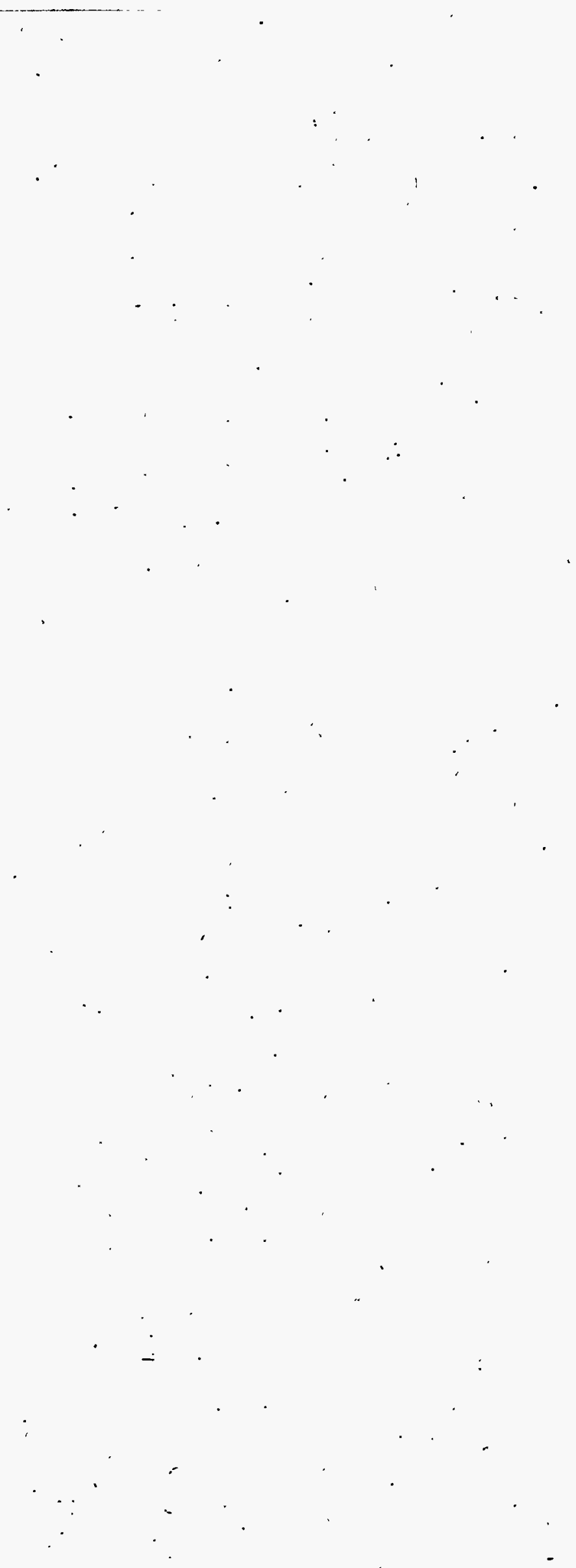




\section{Appendix 5-C: Bibliography}

Abt Associates'Inc. 1991. A Methodology for Evaluating the Costs and Global Warming Implications of Ethanol. Report prepared for Office of Policy Analysis; U.S. Environmental Protection Agency, April 2, 1991.

Chowdhury, A., E.O. Heady, and S. Bhide. 1981. Optimum Crop Production and Resource Use Under Alternative Energy Prices and Agricultural Exports: A Separable and ChangeConstrained Programming Analysis. Report 103. The Center for Agricultural and Rural Development, Ames, IA.

Christensen, D.A., R.J. Schatzer, E.O. Heady, and B.C. English. 1981. The Effects of Increased Energy Prices on U.S. Agriculture: An Econometric Approach. Report 104. The Center for Agricultural and Rural Development, Ames, IA.

Dvoskin, D., E.O. Heady, and B.C. English. 1978. Energy Use in U.S. Agriculture: An Evaluation of National and Regional Impacts from Alternative Energy Policies. The Center for Agricultural and Rural Development. Report 78. Ames, IA.

Energy Information Administration, NEMS Component Design Report for Biofuels (Ethanol) Supply Submodule - Renewable Fuels Model - National Energy Modeling System. Draft $3 / 12 / 93$

English, Smith, Atwood, Johnson, and Oamek. 1989. Resource Conservation Act Analysis; An Overview of the CARD Agricultural Resource Interregional Modeling System. The Center . for Agricultural and Rural Development, Iowa State University, Ames, IA, Technical Report 89-TR11.

Kane, S.M., and J.M. Reilly. 1989. Economics of Ethanol Production in the United States. Agricultural Economic Report 607. USDA/ERS, Washington, 'DC. .

LeBlanc, M. and J. Reilly. 1988. Ethanol: Economic and Policy Tradeoffs. Agricultural Economic Report No. 585. Resources and Technology Division, Economic Research Service, U.S. Department of Agriculture, Washington, DC.

Lee, Hyunok. June 1993. "Ethanol's Evolving Role in the U.S. Automobile Fuel Market." -Industrial Uses of Agricultural Materials, Situation and Outlook Report. United States Department of Agriculture, Washington, DC.

Lee, R., S.M. Cohn, and R.D. Perlack. 1991. Prototype of an Integrated Model for Projecting Biofuels Consumption. Draft report prepared for Energy Information Administration, U.S. Department of Energy. Oak Ridge National Laboratory, Oak Ridge, TN. 
Marland, G. and A.F. Turhollow. 199.1. " $\mathrm{CO}_{2}$ Emissions From the Production and Combustion of Fuel Ethanol from Corn." Energy, 16(11/12):1307-1316.

Solar Energy Research Institute. March 1990. "The Potential of Renewable Energy: An Interlaboratory White Paper," -SERI/TP-260-3674, Golden,.CO, prepared for the Office of Policy, Planning, and Analysis, U.S. Department of Energy, in support of the National Energy Strategy.

Trumble, David A. 1994. Estimation of Supply Curve for Ethanol with Corn as the Feedstock. Draft report prepared for Energy Information Administration, U.S. Department of Energy. Oak Ridge National Laboratory, Oak Ridge, TN.

Turhollow, A.F. 1991. "Economic Consideration for the Production of Wood for Energy." Presentation at Trees for Energy, First National Fuelwood Conference, November 11-13, 1991, Lincoln, NE.

Turhollow, A.F., D.A. Christensen, and E.O. Heady. 1984. The Potential Impacts of Large-Scale Fuel Alcohol Production from Corn, Grain Sorghum, and Crop Residues Under Varying Technologies and Crop Expert Levels. Report 126. The Center for Agricultural and Rural Development, Ames, IA.

Turhollow, A.F., C. Short, and E.O. Heady. 1983. Potential Impacts of Future Energy Price Increases on U.S. Agricultural Production. Report 116. The Center for Agricultural and Rural Development, Ames, IA:

Tyson, K.S. 1990. Biomass Resource Potential of the United States. Report prepared for Office of Transportation Technologies, U.S. Department of Energy, October 1990. Solar Energy Research Institute, Golden, $\mathrm{CO}$. 


\section{Appendix 5-D: Model Abstract.}

\section{Model Name:}

Biomass (Ethanol) Supply Submodule

\section{Model Acronym:}

BSS

\section{Description:}

The BSS is a supply curve model for ethanol used for transportation fuel. It utilizes an ethanol cost function, NEMS energy price data, and outputs from an exogenous agricultural resource allocation linear programming model, to produce ethanol supply curves. The ethanol cost function models the impact of corn feedstock prices and supplies, energy prices, and feedstock conversion . costs on delivered ethanol prices. The BSS's primary interaction is with the NEMS Petroleum Market Module (PMM).

\section{Purpose of the Model:}

The purpose of the Biofuels (Ethanol) Supply Submodule (BSS) is to provide annual corn-derived ethanol supply-cost curves for use by the Petroleum Market Module (PMM) in projecting ethanol requirements. For each year, the BSS calculates delivered ethanol prices for different ethanol demand.levels. The ethanol supply/cost projection information by Petroleum Administration for Defense District (PADD) and by Census Region. These projections are made Through the year 2015. The BSS, as a part of NEMS, help the Energy Information Administration develop forecasts published in its Annual Energy Outlook (AEO).

\section{Most Recent Model Update:}

October 29,1993

\section{Part of Another Model?:}

The Biofuels submodule is a component of the Renewable Fuels Module (RFM) of the National Energy Modeling System (NEMS).

\section{Official Model Representative:}

\section{Laurence Sanders}

Coal, Uranium, and Renewable Fuels Analysis Branch

Energy Information Administration (202) 586-2049

\section{Documentation:}

NEMS Documentation Report: Renewable. Fuels Submodule, March 1994

\section{Archive Media and Installation Manual(s):}

Archived as part of the NEMS production runs. 


\section{Energy System Described:}

Agricultural sector-corn feedstock production net of byproducts; corn feedstock requirements for ethanol production; ethanol as a refinery input for gasoline blending.

\section{Coverage:}

- Geographic: Nine Census Regions:- New England, Mid Atlantic, South Atlantic, East North Central, West North Central, East South Central, West South, Central, Mountain, and Pacific. Five PADD's: Atlantic Coast, North Central, South Central, Mountain, and Pacific

- Time Unit/Frequency: Annual, 1990 through 2015

- Products: Motor Fuel/Additives

\section{Modeling Features:}

NA

\section{Non-DOE Input Sources:}

Omnibus Reconciliation Act of 1990

- $\$ 0.54$ per gallon subsidy for ethanol blenders

Marland \& Turhollow, 1991

- Quantity of energy needed for process conversion

- Quantity of fuel used in the production of feedstocks

United States Department of Agriculture (USDA) - Report \#585

- Capitol \& operating costs for conversion technologies

United States Department of Agriculture (USDA) - (ARIMS)

- Percentage of ethanol produced by PADD's

- Feedstock cost data

Anthony Turhollow, Oak Ridge National Laboratory, Oak Ridge, Tennessee

- Beta coefficients derived from an analysis of ARIMS outputs 


\section{DOE Input Sources:}

- ELA coal prices

- EIA diesel prices

\section{Computing Environment:}

- Hardware Used: IBM 3090

- Operating System: MVS

- Language/Software Used: VS FORTRAN,.Ver. 2.05

- Memory Requirement: $26 \mathrm{~Kb}$

- Storage Requirement: $14 \mathrm{~Kb}$

- Estimated Run Time: 0.02 seconds

- Special Features: None.

Independent Expert Reviews Conducted: None.

\section{Status of Evaluation Efforts by Sponsor:}

None. 



\section{Appendix 5-E: Data Quality and Estimation Processes}

This Appendix provides an overview of the Agricultural Resources Interregional Modeling System (ARIMS), a main source of input data used in the BSS.

\section{. Agricultural Resources Interregional Modeling System (ARIMS)}

The primary purpose of the national Agricultural Resource Interregional Modeling System , (ARMMS) is as a system to analyze agricultural policies. Because resources and agricultural production practices differ by region, numerous regional attributes and responses to agricultural and resource policies can be evaluated in ARTMS. For examplè; policies can be evaluated that impact regional resource availability, farming techniques, resource prices, input availability, alternative levels of demand, and environmental allowances. The foremost use of the ARIMS, however, is to appraise future agricultural resource requirements.

ARIMS is a large linear programming model that includes numerous input coefficients and constraints. The inputs include projections of future resource availability, future demand levels and regional distribution of those demands, future commodity yields, and future changes in the ability of farmers to produce agricultural commodities. These projections are then used in a programming model and the composite effect of these individual impacts and the policy provisions introduced is analyzed.

The linear programming model of the agricultural sector is a set of mathematical relationships incorporating "characteristics most relevant to agricultural production. The model minimizes the cost of producing and transporting agricultural commodities as required to meet pre-specified demands.

ARIMS divides the Nation into eight economic sectors. These eight sectors, integrated by a linear programming framework, are designed to represent the production processes and driving forces of U.S. agriculture.

- The modeling system incorporates three different regional definitions. Production of agricultural commodities includes 105 Crop Producing Areas and 31 Livestock Producing Areas. The grazing production sector is specified for a third set of regions-34 ecosystems. The 31 Livestock Producing Areas also serve as the regional structure for non-water input, purchases. In addition to the regions explicitly contained in the model structure, coefficients are developed by county, State, USDA farm production region and Major Land Resource Area.

Livestock markets (cattle, hogs, poultry) have an important role in determining the supply price of ethanol. The vast bulk of grain produced in the United States is fed to livestock, and the by- 
products of producing ethanol from corn (gluten meal, gluten feed, and distillers dried grains) are either fed to domestic livestock or exported. The by-products; including corn oil, from converting corn into ethanol are typically valued at about 50 percent of the raw corn cost.

The value of the by-products is directly proportional to the caloric and-protein values of the feed by-products. Gluten meal ( 60 percent protein) and gluten feed ( 20 percent or more protein) are high in protein relative to corn ( 9 percent protein) and thus have a strong. impact on soybean meal prices and vice versa. Soybean meal is 44 percent or more protein and is the main protein supplement for livestock. This competitive interaction is captured in the ARIMS model. As ethanol production from corn increases, the unit value of the feed by-products tends to decrease.

As an example, with corn at $\$ 2.50$ per bushel the by-products are worth $\$ 1.25$ per bushel, so the net feedstock cost is only $\$ 1.25$ per bushel. At a conversion rate of 2.5 gallons of ethanol per bushel of corn, the net feedstock cost is only $\$ 0.50$ per gallon instead of $\$ 1.00$ per gallon.

The only use of ARIMS in this submodule is as a source of feedstock cost data. Regression equations relating the cost of corn to energy input prices were estimated from successive runs of the model. However, the summary statistics of those regressions are not currently available as they were conducted off-site and were not included in subsequent reports. For the next AEO, data will be obtained and analysis performed in order to estimate error terms and other relevant statistical information. ARIMS is run exogenously to NEMS, and is therefore not an integrated : component of the BSS. 


\title{
6. Wood Submodule
}

\author{
Model Purpose
}

\section{Model Objectives}

The purpose of the Wood Submodule is to furnish cost and performance characteristics of a wood burning electricity generating technology to the Electric Market Module (EMM) of the National Energy Modeling System (NEMS). The submodule utilizes a regional wood supply schedule from which the wood price is determined. The wood supply schedule is based on the accessibility of wood resources by the consuming sectors from existing timber resources excluding future wood energy crops.

The basic data inputs to the submodule include:

- wood resource inventory and cost data from the U.S. Forest Service,

- wood distribution and preparation cost data,

- annual wood energy consumption by sector,

- conversion factors for transforming (1) wood tonnage information into Btu energy . equivalents, (2) U.S. Forest Service wood inventory region data into Census Division data, and (3) Census Division wood prices into NEMS Electricity Region prices,

The Submodule's outputs consist of (1) wood energy conversion performance values, (2) operating and maintenance costs (fixed and variable) by Census Division, NERC Region and year, and (3) capital costs for a wood-fired power plant. Prices of short rotation biomass (woody and herbaceous) energy crops are not currently reflected in the wood supply schedule.

\section{Relationship of the Wood Submodule to Other Models}

The Wood Submodule interacts with other NEMS modules by both accepting inputs and passing outpuits via the Renewable Fuels Model (RFM) shell.It does not interact with other submodules in the RFM. Regional wood consumption data from the commercial, industrial, and electricity modules are used in the wood module to determine the regional wood supply price. A total capacity potential is calculated from regional supply curve data and each year, the accumulated capacity from the EMM is measured against this limit and constraint if it exceeds the limit.

The Wood Submodule outputs include wood prices consistent with the quantities demanded. These are supplied to the EMM to determine capacity, dispatched power, and electricity prices. 


\section{Modeling Rationale}

\section{Theoretical Approach}

The wood submodule provides regional wood supply curves for noncaptive markets. The supply curves are based on a detailed analysis of historical wood consumption for a set of wood sources in the industrial, electric generating, commercial, and residential sectors:

Prior to the development of NEMS, electricity from wood was estimated by EIA as a series of econometric equations based on historical wood use from various sources and the relationship between wood consumption and key variables. For utilities, projections of wood facility capacity and capacity utilization factors were made off-line and included in the EMM. No competition between wood and other sources of electricity generation was modeled; rather, the use of wood decreased the requirements from other sources of generation.

The wood use in NEMS is decomposed and modeled as two distinct markets, the captive and noncaptive wood markets. The captive market pertains to users with dedicated wood supplies that combust wood byproducts resulting from the manufacturing process (i.e., the pulp and paper and forest products industries): The wood waste combustion in captive markets serves the dual role of energy supplier and waste disposal method. The captive wood market is modeled by the industrial module of NEMS:

The noncaptive wood market is represented in the Wood Submodule of the RFM. The noncaptive market is defined to include the commercial and electric utility sectors, as well as the noncaptive portion of the industrial sector. It is necessary to include commercial and industrial consumption in order to properly estimate supply and demand conditions, as these represent alternative economic uses of the wood supply. There is an additional noncaptive market serving residential uses of wood. This market is modeled in the residential demand module.

Because of the scarcity of reliable data and the relatively small size of the noncaptive market, EIA decided to develop a fairly simple model structure consisting of one supply schedule per region. This schedule defines the quantity and cost relationships of wood resources accessible by all nonciative, non-residential consumers; it is based on an off-line data accounting procedure that aggregates supply/price information from several U.S. .Forest Service (U.S.F.S.) wood rèsource inventory and price surveys.

\section{Wood Classification}

The total wood supply consists of distinct resources which are represented in aggregated regional supply schedules. The U.S.F.S. has divided wood resources into 15 categories (Table 4). Ten of the categories supply the commercial, industrial and utility sectors. Black liquor is used within the pulp and paper industry, a "captive" industry. 
Table 4. U.S. Forest Service Wood Resource Categories by Type and Demanding Sectors

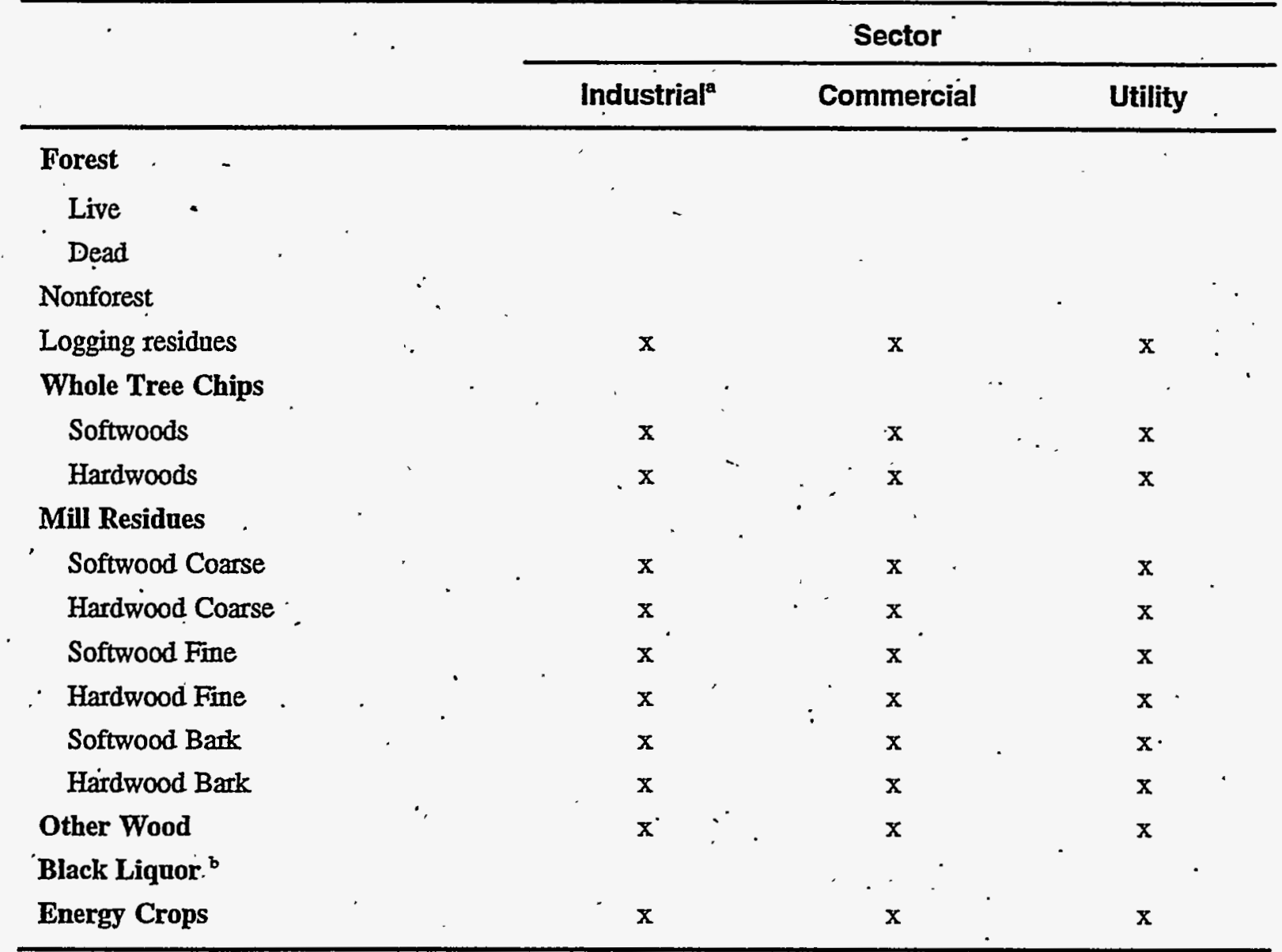

${ }^{2}$ Noncaptive industrial.

Black liquor is only used by the pulp and paper industry.

\section{Fundamental Assumptions}

A basic assumption of the Wood Submodule is that the supply price for noncaptive wood energy is the same across all sectors. This assumption allows the construction of a single supply schedule for all sectors to yield a supply price for the electric utility sector.

Another important fundamental assumption relates to the treatment of wood transportation costs. The difficult aspect of building supply curves for wood is modeling the economic accessibility to the resource, rather than estimating the physical amount of wood that can be used. This submodule assumes a fixed "typical" transportation distance in calculating costs. Because no interregional wood trade exists, it is assumed that no wood is transported among NEMS regions. 
The wood supply analysis was conducted in 1984 and is based on 1976 forest inventory data. Logging residue and whole tree data are obtained from an unpublished manuscript by McQuillan, et al., in which the authors projected waste wood inventory and retrieval costs from 1990 through 2030. Forest management has changed dramatically since the study was undertaken in 1984. Recently, the practice of clear-cutting in old-growth forests has come under scrutiny for its impacts on endangered species such as the Northern spotted owl. It appears increasingly likely that large portions of the remaining old-growth forest will be set aside as wildlife preserves, and governiments will probably place new restrictions on activities in second-growth federal and state forests. These new environmental restrictions will reduce the softwood supply and lead to more intensive use of existing waste wood inventories for furniture making, construction and other uses, resulting.in higher prices and smaller inventories than projected by the authors. Working to mitigate this trend are restrictions on raw log exports from federal and state lands, increasing the proportion of logs from federal and state lands that are milled domestically.

\section{Alternațive Approaches}

As mentioned above, the Wood Submodule is based on the simplifying assumption that a single regional supply schedule for all wood resources is appropriate for the electric generating sector. However, this simplification may not be able to capture all of the important dynamics in wood markets. Wood costs increase rapidly as the distance transported increases. Wood is used relatively near its source, unlike coal, gas, or oil. A concentrated use in a small.area is difficult to represent in the large regions of NEMS, where the average wood share of energy used could be quite small. Large-scale facilities could lead to transportation problems (e.g., too many trucks required for delivering wood). While this submodule estimates supply curves for the production of wood, the transportation distance of a facility from the wood supply can make up a significant share of the delivered cost. In this module, a typical transportation distance for each Census Division is assumed. A more complete representation would include transportation supply curves.

\section{Wood Submodule Structure}

\section{Data Analysis}

The Wood Submodule's compitational procedures consist of two basic routines: derivation of the all-sector supply schedule and aggregation of all quantities and prices into single sectoral values. This section outlines the procedures and equations associated with these two routines. 


\section{All-Sector Supply Schedule}

The regional wood energy quantities for the all-sector supply schedule, $Q_{\text {total, },}$, are calculated as the sum of wood energy quantities for each wood resource available in each Census division $\mathrm{R}$ :

$$
\begin{aligned}
& Q_{\text {total } R}=\cdot\left(Q_{\text {hardwood chips }, R}+Q_{\text {softwood chips }, R}\right) \\
& +\left(Q_{\text {hardwood logging residue, } R}+Q_{\text {soffwood logging residue, } R}\right) \\
& +\left(Q_{\text {course mill residue,R }}+Q_{\text {fine mill resizue,R }}+Q_{\text {bark mill residue, } R}\right) \\
& +Q_{\text {other wood, } R}
\end{aligned}
$$

The wood quantities and associated prices are aggregated using a Lotus 1-2-3 spreadsheet. The following sections outline the processing steps associated with the aggregation of quantity and price data for each regional supply schedule wood resource. These processing steps are performed manually off-line.

\section{Supply Schedule Processing Steps: Whole Tree Chips and Logging Residues}

1. Data for whole tree chips and logging residues are obtained from U.S. Forest Service projections. There are separate inventories for hardwoods and softwoods.

2. Price data are converted from 1980 dollars per thousand cubic feet to 1987 dollars per million Btu based on the implicit GNP price deflator of 85.7 in 1980 and $117: 4$ in 1987. The density of softwood is assumed to be 35 pounds per cubic feet. For hardwood a density of 40 pounds per cubic feet is assumed. Quantity data are converted from million cubic feet to trillion Btu using the density for hardwood and softwood, and assuming 15-percent moisture and a heat content of 17 million Btu per dry ton of wood. ${ }^{13}$ -

3. Whole tree chip supply data are represented as an inventory. Annual supplies must be obtained by allocating the inventory according to the rate of annual sustainable harvesting of the resource. A constant annual rate of 5 percent of the total inventory supply is assumed. Logging residue data is already presented as annual supply quantities, so no adjustments are necessary. ${ }^{14}$.

4. The supply schedules for hardwood and softwood are combined with the use of a price lookup algorithm. A price (and associated quantity) from either the hardwood or softwood schedule is first selected. The price from the other wood schedule that is -closest to but.not greater than the selected price is used as the other lookup price. The quantities associated with the two lookup prices are then added together. This lookup

${ }^{13}$ Tumollow, et al., Oak Ridge National Laboratory, "Data and Sources, Biomass Supply, Draft, 1993.

${ }^{14}$ Ibid. 
algorithm results in wood energy supply quantities for all price points represented by both hardwood and softwood supply schedules.

5. Data is then mapped to Census divisions using factors based on the 1987 wood inventory data obtained from the U.S. Forest Service.

\section{Supply Schedule Processing Steps: Mill Residues}

1. Mill residue data for 1987 is obtained from U.S. Forest Service for three types: coarse .wood, fine wood, and bark. Each type of mill residue is divided into four uses: fiber, fuel, other, and unused. The fiber and "other". categories are not used for energy production.

2. The amount of fine wood and bark residues that are used for fuel in captive markets are excluded. Fine wood and bark residues available to the nonresidential, noncaptive market are estimated to be 10 percent of the total fine wood/bark residue supply. This percentage was obtained by assuming that the nonresidential, noncaptive share of the total wood supply ( 8.7 percent) is a good proxy for the nonresidential, noncaptive share of the fine wood/bark residue supply. ${ }^{15}$

3. The Forest Service estimates that 42.9 trillion Btu of mill residues are not used at all. It is arbitrarily assumed that 50 percent of this quantity is available for fuel. ${ }^{16}$

4. The total quantity of each type of residue is determined from the percentages of wood available and the quantity. data provided by U.S.F.S..

5. Quantities are converted from thousand tons at 12 percent moisture to trillion Btu based on the conversion of 17 million Btu per dry ton. ${ }^{17}$

6. U.S. Forest Service mill residue data are disaggregated into three supply regions: North, South, and West. Wood inventory data for 1987 is used to map the three supply regions into Census Divisions. It is conceptually better to use mill residue consumption by state for Census Division mapping purposes, but such data are unavailable.

7. The quantities from each type of residue are added together to provide an overall quantity for each Census division. .

8. Price data for mill residues were obtained from the U.S. Forest Service. Prices for each type of residue are obtained for both hardwood and softwood.

${ }^{15}$ Ibid.

${ }^{16}$ Tbid.

${ }^{17}$ Ibid. 
9. The prices of hardwood and softwood are combined to form a weighted average price for each type of residue.

10. Prices are converted form 1986 dollars per cubic meter to million Btu based on the following assumptions: the GNP price deflator for 1986 is -113.8 and 117.4 for 1987; there are 35.29 cubic feet in a cubic meter; and one cubic foot is 37.5 pounds at 12 pércent moisture.

11. A transportation cost was added to get a delivered price based on an assumed trip of 20 miles, a cost of $\$ 0.14$ per dry ton mile. The prices are then mapped to Census divisions.

\section{Supply Schedule Processing Steps: Other Wood}

1. Data on other wood use (including furniture, construction debris, waste pallets, and demolition wastes) are obtained from the U.S. Forest Service.

2. Price data are converted from 1986 dollars per cubic feet to 1987 per million Btu based on the following assumptions: the GNP price deflator is 113.8 in 1986 and 117.4 in 1987; there are 35.29 cubic feet in a cubic meter; there are 37.5 pounds per cubic foot. at 12 percent moisture; and there are 17 million Btu per dry ton.

3. The quantity of other wood is converted from million cubic feet to trillion Btu. The price data and quantity are then mapped to Census divisions using 1990 population estimates.

\section{Key Computations and Equations}

The wood submodule consists of one FORTRAN subroutine. It computes the regional wood supply price given the current regional wood consumption passed from the industrial, commercial, and electric generating modules. The wood price is added to the variable operating cost and passed to the Electricity Planning Submodule (ECP) along with other cost-performance figures (i.e. capital cost, fixed operation and maintenance cost, capacity factors, and heat rates).

The wood quantity-price relations are implemented in a matrix representing the supply curve as step functions. A linear interpolation scheme is used to determine the wood price given a wood quantity.

Since the quantity-price relations are established for Census regions, and the cost and performance characteristics of the biomass technology are defined for NERC regions, a geographic mapping was necessary to generate wood prices by NERC regions (Table 6A-2).

In addition to the assignment of cost performance characteristics, the wood submodule passes the maximum available electricity generating capacity using wood to the ECP. This capacity limit 
is computed by decremanting the initial total potential by already installed capacity and for each . subsequent year, decrementing the last year's unplanned new capacity form the previous limit. The initial total generating capacity for each region is determined by dividing the maximal quantity of wood reserves in the supply curve by the heat rate, the capacity factor, and 8760 as the number of hours per year.

The technology represented by the cost and performance values for new capacity is the Integrated Gasification Combined Cycle (IGCC) system for wood. The unit cost is modular and capable of being shop fabricated. The cost values include storage and wood handling, magnetic separators, and ash handling equipment. The gasifier is equipped with solid and gas recycling systems. A modular hot gas filtration unit is included in the cost assumptions.

The procedural execution of the wood subroutine is illustrated in Figure 5. 
Figure 5. Wood Submodule Flowchart

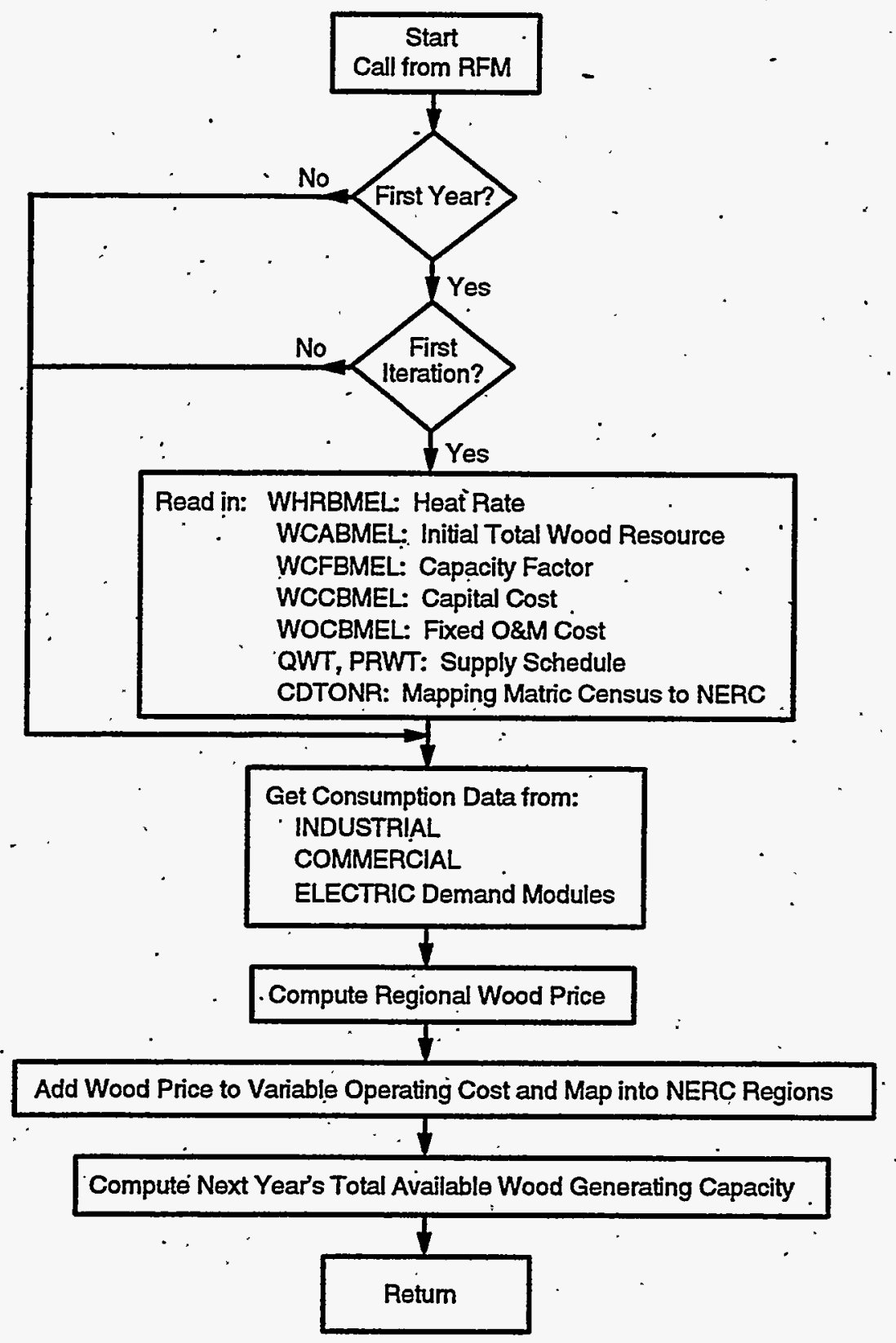





\section{Appendix 6-A: Inventory of Variables, Data; and Parameters}

Appendix 6-A provides information on variables used in the Wood Submodule. Table 6A-1 gives a complete listing of all variables including definitions and dimensions, sources, measurement units, and page references. Variables are classified as Submodule data inputs, calculated variables, and Submodule outputs. Following Table 6A-1 are detailed descriptions of each input datà item.

Table 6A-1: NEMS Wood Submodule Inputs and Variables

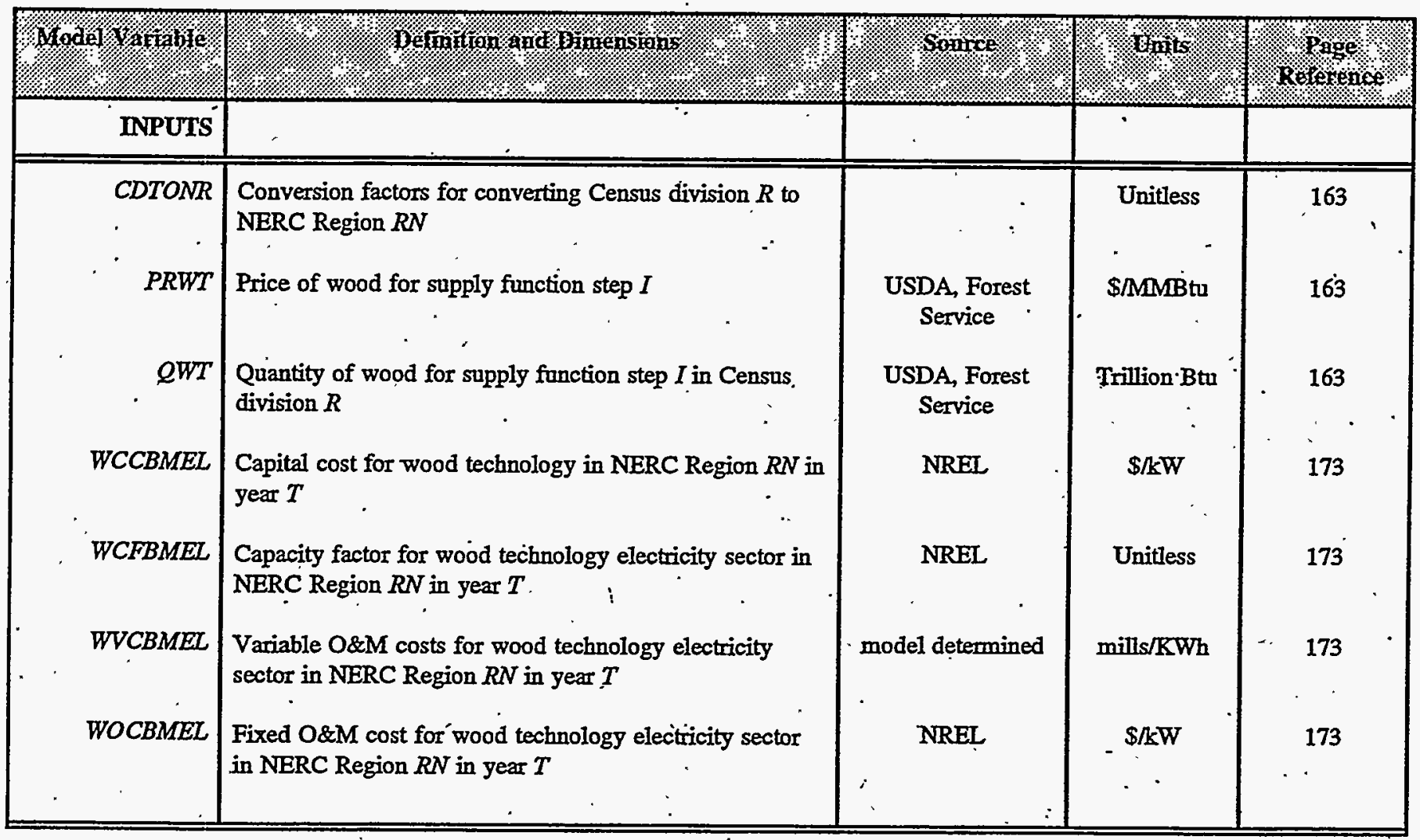


Table 6A-1. NEMS Wood Submodule Inputs and Variables (Continued)

\begin{tabular}{|c|c|c|c|c|}
\hline 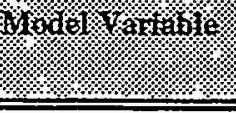 & Whan & 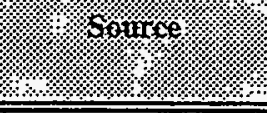 & Wy & oxtratenco \\
\hline VARIABLES & & & & \\
\hline $\begin{array}{l}\text { PELCM } \\
\text { PELIN } \\
\text { OBMCM } \\
\text { OBMEL } \\
\text { OBMWN } \\
\text { QUICI }\end{array}$ & $\begin{array}{l}\text { Price of electricity in the commercial sector in Census } \\
\text { division } R \text { and year } T-1 \\
\text { Price of electricity in the industrial sector in Census } \\
\text { division } R \text { and year } T-1 \\
\text { Quantity of wood consumed in the commercial sector in } \\
\text { Census division } R \text { in year } T \\
\text { Quantity of wood consumed by utilities in Census } \\
\text { division } R \text { in year } T \\
\text { Quantity of wood consumed in the industrial.sector in } \\
\text { Census division } R \text { in year } T \\
\text { Quantity of wood consumed by all sectors }\end{array}$ & $\begin{array}{l}\text { NEMS Commercial } \\
\text { Module : } \\
\text { NEMS Industrial } \\
\text { Module : } \\
\text {. } \\
\text {. }\end{array}$ & $\begin{array}{l}\$ / k W h \\
\$ / k W h \\
\text { MMBtu } \\
\text { MaMBtu } \\
\text { MMBtu } \\
\text {. } \\
\text { MMBtu }\end{array}$ & $\begin{array}{c}177 \\
\cdot \\
\cdot 177 \\
\cdot \\
177\end{array}$ \\
\hline OUTPUTS & & & & . \\
\hline $\begin{array}{c}\text { WOCBMEL } \\
P B M C M \\
\text { PBMEL } \\
\text { PBMIN } \\
\text { PUCI }\end{array}$ & $\begin{array}{l}\text { Capacity for utilities in NERC Region } R N \text { in year T1 } \\
\text { Capital cost for wood technology in NERC Region } R N \text { in } \\
\text { year } T \\
\text { Capacity factor for wood technology electricity sector in } \\
\text { NERC Region } R N \text { in year } T \text {. } \\
\text { Variable O\&M costs for.wood technology electricity } \\
\text { sector in NERC Region } R N \text { in year } T \\
\text { Fixed O\&M cost for wood technology electricity sector } \\
\text { in NERC Region } R N \text { in year } T \\
\text { Price of wood for the commercial sector in Census } \\
\text { division } R \text { in year } 1 \\
\text { Price of wood for utilities in Census division } T \text { in year. } \\
T 1 \\
\text { Price of wood for the industrial sector in Census division } \\
R \text { in year. } T 1 \\
\text { Price of wood for all sectors }\end{array}$ & $\begin{array}{r}\text { NREL } \\
\text { NREL } \\
\text { NREL } \\
\text { NREL }\end{array}$ & $\begin{array}{l}\text { Unitless } \\
\text { mills/KWh } \\
\$ / \mathrm{kW} \\
\$ / M M B \operatorname{m} \\
\text { \$/MMBtu } \\
\text { \$/MMBtu } \\
\text { \$/MMBtu }\end{array}$ & $\begin{array}{r}177 \\
177 \\
177 \\
177\end{array}$ \\
\hline
\end{tabular}


Because the available wood resource data are structured by cencus regions and NEMS is structured by a variation of NERC regions, it is necessary to provide for that transition. This is done in a matrix in which estimates of wood quantities for each census region are allocated to the NERC regions

The resulting conversion factors are listed in the following table:

Table 6A-2. Map of Census Divisions to NERC Regions

\begin{tabular}{|c|c|c|c|c|c|c|c|c|c|c||}
\hline & \multicolumn{10}{|c|}{ Census Division } \\
\hline NERC Region & 1 & 2 & 3 & 4 & 5 & 6 & 7 & 8 & 9 & 10 \\
\hline 1 & 0 & 0.06 & 0.90 & 0 & 0.12 & 0.23 & 0 & 0 & 0 & 0 \\
\hline 2 & 0 & 0 & 0 & 0 & 0 & 0 & 0.03 & 0 & 0 & 0 \\
\hline 3 & 0 & 0.36 & 0 & 0 & 0 & 0 & 0 & 0 & 0 & 0 \\
\hline 4 & 0 & 0 & 0 & 0.05 & 0 & 0 & 0 & 0 & 0 & 0 \\
\hline 5 & 0 & 0 & 0.10 & 0.05 & 0 & 0 & 0 & 0 & 0 & 0 \\
\hline 6 & 0 & 0.58 & 0 & 0 & 0 & 0 & 0 & 0 & 0 & 0 \\
\hline 7 & 1.00 & 0 & 0 & 0 & 0 & 0 & 0 & 0 & 0 & 0 \\
\hline 8 & 0 & 0 & 0 & 0 & 0.08 & 0 & 0 & 0 & 0 & 0 \\
\hline 9 & 0 & 0 & 0 & 0 & 0.80 & 0.65 & 0 & 0 & 0 & 0 \\
\hline 10 & 0 & 0 & 0 & 0.90 & 0 & 0.12 & 0.97 & 0 & 0 & 0 \\
\hline 11 & 0 & 0 & 0 & 0 & 0 & 0 & 0 & 0.70 & 0.90 & 0 \\
\hline 12 & 0 & 0 & 0 & 0 & 0 & 0 & 0 & 0.30 & 0 & 0 \\
\hline 13 & 0 & 0 & 0 & 0 & 0 & 0 & 0 & 0 & 0.10 & 0 \\
\hline 14 & 0 & 0 & 0 & 0 & 0 & 0 & 0 & 0 & 0 & 0 \\
\hline 15 & 0 & 0 & 0 & 0 & 0 & 0 & 0 & 0 & 0 & 0 \\
\hline 16 & 0 & 0 & 0 & 0 & 0 & 0 & 0 & 0 & 0 & 0 \\
\hline & & & & & & & & 0 & 0 \\
\hline
\end{tabular}

MODEL INPUT: CDTONR

DEFINITION: - Conversion factors for converting Census division $R$ to NERC Region $R N$

SOURCE: Oak Ridge National Laboratory, "Data and Sources Biomass Supply", Draft prepared for EIA under Contract No. DE-AC05-84OR21400, Oak Ridge, TN, June 27, 1993. 


\section{MODEL INPUT: $\quad P R W T$ and $Q W T$.}

DEFINITION: Price and quantity from the all-sector supply schedule for supply function step $I$

$P R W T$ and $Q W T$ represent the price quantity relation for a wood composite consisting of the following wood types: 1) .whole tree chips, 2) logging residues, 3) mill residues, and 4) other wood. Data on each wood type are collected and compiled individually and then combined to one all-sector wood supply schedule. The supply schedule is shown in Table 6E-1.

\section{Whole Tree Chips}

The wood resource designated for whole tree chips are estimated from timber inventory data collected and compiled by McQuillan, et al. (1984). The availablity of wood for whole tree chipping is based on a sustainable timber cutting cycle of 20 years. This means that on a sustainable basis, $1 / 20$ of the inventory is available in any one year.

The inventory distinguishes between softwoods and hardwoods in nine U.S.F.S. regions. McQuillan et al. reported the prices-as a delivered supply price to large users which does not include the additional cost for wood drying.

The quantities are mapped from the U.S.F.S. regions to census divisions using a mapping matrix for whole tree chips.

Tables 6A-3a, 6A-3b; and 6A-3c show the wood supply schedule for whole tree chips. Under each price.level, the quantity of material in each region at that price is indicated. 
Table 6A-3a. Supply Schedule for Whole Tree Chips by Census Region (in 1987 Dollars/Million Btu and Trillion Btu)

\begin{tabular}{|c|c|c|c|c|c|c|c|c|c|c|c|}
\hline \multirow[t]{2}{*}{ • } & \multicolumn{11}{|c|}{ - $1987 \$$ Million Btu } \\
\hline & 0.542 & 0.711 & 0.813 & 0.948 & 1.083 & 1.185 & 1.354 & 1.422 & 1.625 & 1.659 & 1.896 \\
\hline Region & 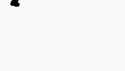 & & & & & Ilion B & & & - & & \\
\hline New England & 0.0 & 0.0 & 0.0 & 0.0 & 0.0 & 0.0 & 0.0 & 3.0 & 5.9 & $15.9^{\circ}$ & 58.1 \\
\hline Middle Atlantic & 0.0 & 0.0 & 0.0 & 0.0 & 0.0 & 0.0 & 0.0 & $\quad 3.1$ & 6.1 & 16.4 & 60.0 \\
\hline $\begin{array}{l}\text { East North } \\
\text { Central }\end{array}$ & 0.0 & 0.0 & 0.0 & 0.2 & 0.2 & 0.2 & 0.2 & 7.2 & 8.5 & 30.6 & 86.1 \\
\hline $\begin{array}{l}\text { West North } \\
\text { Central }\end{array}$ & 0.0 & 0.0 & 0.0 & 0.1 & 0.1 & 0.1 & 0.1 & 4.0 & 4.8 & 16.8 & 47.4 \\
\hline South Atlantic. & 0.0 & 0.0 & 0.0 & 0.0 & 0.0 & 67.4 & 75.4 & 219.7 & 233.1 & 300.7 & 334.9 \\
\hline $\begin{array}{l}\text { East South } \\
\text { Central }\end{array}$ & 0.0 & 0.0 & 0.0 & 0.0 & 0.0 & 9.1 & 9.7 & 45.4 & 48.6 & 118.4 & 186.3 \\
\hline $\begin{array}{l}\text { West South } \\
\text { Central }\end{array}$ & 0.0 & 0.0 & .0 .0 & 0.0 & 0.0 & 8.1 & 8.6 & 39.2 & 41.7 & 99.7 & 149.1 \\
\hline Mountain & 0.0 & 0.0 & 0.0 & 0.0 & 0.0 & 0.2 & 1.6 & 5.0 & 13.9 & 21.0 & 58.2 \\
\hline Pacific & 0.0 & 0.0 & 0.0 & 0.0 & 0.0 & 0.0 & 6.9 & 8.6 & 38.0 & 45.6 & 90.7 \\
\hline Total & 0.0 & 0.1 & 0.1 & 0.3 & 0.4 & 85.1 & 102.5 & 335.1 & 400.7 & 665.0 & 1071.1 \\
\hline
\end{tabular}


Table 6A-3b. Supply Schedule for Whole Tree Chips by Census Region (in 1987 Dollars/Million Btu and Trillion Btu)

\begin{tabular}{|c|c|c|c|c|c|c|c|c|c|c|c|}
\hline & \multicolumn{11}{|c|}{ 1987\$MVillion Btu } \\
\hline & .2 .133 & 2.167 & 2.370 & 2.438 & 2.709 & $2.84 \overline{4}$ & 3.250 & 3.318 & 3.792 & $\begin{array}{c}1 \\
4.266\end{array}$ & 4.334 \\
\hline Region & \multicolumn{11}{|c|}{ Trillion Btu } \\
\hline New England & 84.6 & 87.9 & 93.7 & 99.8 & 101.8 . & 109.4 & 109.9 & 115.4 & 140.6 & 217.6 & 252.0 \\
\hline Middle Atlantic & 87.3 & $90.7^{\circ}$ & 96.7 & 103.1 & 105.1 & 113.0 & 113.5 & $119.1^{\circ}$ & 145.1 & 224.7 & 260.2 \\
\hline $\begin{array}{l}\text { East North } \\
\text { Central }\end{array}$ & 123.5 & 125.4 & 126.5 & 127.4 & 127.8 & 133.4 & 133.5 & $136.8^{\circ}$ & $\begin{array}{l}\cdot \\
161.6\end{array}$ & 338.6 & 378.6 \\
\hline $\begin{array}{l}\text { West North } \\
\text { Central }\end{array}$ & 68.2 & $69.5^{\circ}$ & .70 .2 & 71.1 & $71.4^{\circ}$ & 74.5 & 74.6 & 76.4 & 90.3 & 187.3 & 209.7 \\
\hline South Atlantic & 348.3 & 350.3 & 356.9 & 360.0 & 361.4 & 561.8 & 684.3 & 1169.7 & 1284.4 & 1323.2 & 1339.8 \\
\hline $\begin{array}{l}\text { East South } \\
\text { Central }\end{array}$ & 206.4 & 208.0 & 213.1. & 214.0 & 215.2 & 267.9 & 294.6 & 5427 & 698.5 & 739.0 & 753.1 \\
\hline $\begin{array}{l}\text { West South } \\
\text { Central }\end{array}$ & 159.5 & 160.5 & 164.9 & 165.5. & 166.5 & 212.5 & 236.4 & 457.6 & 5920 & 592.2 & 596.7 \\
\hline Mountain & 61.4 & 79.9 & 81.1 & 101.6 & 106.8 & 111.8 & .118 .6 & 122.5 & 183.3 & 213.2 & 259.8 \\
\hline Pacific & 100.0 & 118.6 & 125.8 & $\cdot 134.8$ & $\therefore 146.0$ & 153.7 & 218.6 & 234.4 & 379.3 & 388.8 & 414.5 \\
\hline Total & 1239.2 & 1290.8 & 1328.9 & 1377.3 & 1402.0 & 1738.0 & 1984.0 & $2974.6^{\circ}$ & 3675.2 & 4224.5 & 4464.6 \\
\hline
\end{tabular}


Table 6A-3c. Supply Schedule for Whole Tree Chips by Census Region (in 1987 Dollars/Million Btu and Trillion Btu)

\begin{tabular}{||l|c|c|c|c|c||}
\hline & $\cdot 5.5$ & \multicolumn{5}{|c||}{ 1987\$/Million Btu } \\
\hline.- & 4.740 & 4.876 & 5.214 & 5.417 & 5.688 \\
\hline \multicolumn{1}{|c|}{ Region } & \multicolumn{5}{|c||}{ Trillion Btu } \\
\hline New England & 278.4. & 303.0 & 305.2 & 305.8 & 305.9 \\
\hline Middle Atlantic & 287.4 & 312.8 & 315.1 & 315.7 & 315.8 \\
\hline $\begin{array}{l}\text { East North } \\
\text { Central }\end{array}$ & 443.2 & 465.7 & 465.8 & 465.8 & 465.8 \\
\hline $\begin{array}{l}\text { West North } \\
\text { Central }\end{array}$ & 245.1 & 258.8 & 258.9 & 259.1 & 259.1 \\
\hline South Atlantic & 1352.6 & 1364.4 & 1365.5 & 1365.8 & 1365.8 \\
\hline $\begin{array}{l}\text { East South } \\
\text { Central }\end{array}$ & 767.8 & 773.0 & 773.0 & 773.0 & 773.0 \\
\hline $\begin{array}{l}\text { West South } \\
\text { Central }\end{array}$ & 596.7 & 596.8 & 596.8 & 596.8 & 596.8 \\
\hline Mountain & 261.4 & 321.4 & 322.6 & 333.6 & 333.7 \\
\hline Pacific & 423.3 & 456.8 & 460.1 & 462.1 & 462.1 \\
\hline Total & 4655.9 & 4852.8 & 4862.9 & 4877.6 & 4878.0 \\
\hline
\end{tabular}

\section{Logging Residues}

The data on available logging residues are also obtained from the report by McQuillan et al. (1984)..Logging residues in this report are listed for softwoods and hardwoods separately in each of the nine U.S.F.S. regions. The price data represent the price for the wood including delivery to large users. They do not include the additional cost for drying.

The supply schedule for softwoods and hardwoods are combined for each U.S.F.S. region and shown in Tables $6 \mathrm{~A}-4 \mathrm{a}$ and $6 \mathrm{~A}-4 \mathrm{~b}$. The structure of this table is similar to that for whole tree chips, however, the prices start at a slightly higher level. 
Table 6A-4a. Total Logging Residue Supply Schedule by Census Regions (in 1987 Dollars/Million Btu and Trillion Btu)

\begin{tabular}{|c|c|c|c|c|c|c|c|c|c|c|c|c|}
\hline & \multicolumn{12}{|c|}{ 1987\$Million Btu } \\
\hline & 1.185 & 1.354 & 1.422 & 1.625 & 1.659 & 1.896 & 2.133 & 2.167 & 2.370 & 2.438 & 2.709 & 2.844 \\
\hline Region & 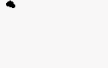 & $\cdot$ & & & Trilli & A Btul & & & & & & \\
\hline New England & 0.0 & 0.0 & 0.0 & 0.0 & 0.0 & 4.8 & 12.8 & 15.4 & 15.4 & 15.4 & 16.2 & 19.0 \\
\hline $\begin{array}{l}\text { East North } \\
\text { Central }\end{array}$ & 0.0 & 0.0 & 0.0 & 0.0 & 0.7 & 2.9 & 11.4 & 11.7 & 11.7 & 11.7 & 11.8 & 14.6 \\
\hline $\begin{array}{l}\text { West North } \\
\text { Central }\end{array}$ & 0.0 & 0.0 & 0.0 & 0.0 & 0.4 & 1.6 & 6.2 & 6.4 & 6.4 & 6.6 & 6.7 & 8.2 . \\
\hline $\begin{array}{l}\text { West South } \\
\text { Central }\end{array}$ & 0.0 & 0.0 & 1.8 & 4.6 & 10.0 & 22.2 & 25.0 & 26.2 & 26.2 & 26.2 & 26.2 & 26.2 \\
\hline Mountain & 0.0 & 0.0 & 0.0 & 0.3 & 0.3 & $2.0^{\circ}$ & 2.3 & 5.0 & $5: 0$ & 10.7 & 12.5 & 12.5 \\
\hline Pacific & 0.0 & 0.0 & 0.0 & 4.3 & 4.6 & 19.4 & 20.8 & 36.8 & 37.6 & .41 .9 & 41.9 & 42.2 \\
\hline Total & 0.0 & 2.3 & $24.5^{\circ}$ & 41.5 & 79.0 & 161.0 & $204 \dot{0}$ & 232.4 & 233.2 & 243.3 & 247.4 & 259.5 \\
\hline
\end{tabular}


Table 6A-4b. Total Logging Residue Supply Schedule by Census Regions (in 1987 Dollars/Million. Btu and Trillion Btu)

\begin{tabular}{|c|c|c|c|c|c|c|c|c|c|c|}
\hline . & , & & & & $987 \$ \mathrm{M}$ & ion Btt & & 1 & & \\
\hline . & 3.250 & 3.318 & 3.792 & 4.266 & 4.334 & 4.740 & 4.876 & 5.214 & 5.417 & 5.688 \\
\hline Region & \multicolumn{8}{|c|}{ Trillion Btu } & - & \\
\hline New England & 19.0 & 19.0 & $19.0^{\circ}$ & $19.0^{\circ}$ & 19.0 & 19.0 & 19.0 & 19.6 . & 19.8 & $21.1^{\circ}$ \\
\hline Middle Atlantic & 19.6 & 19.6 & -19.6 & 19.6 & 19.6 & 19.6 & 19.6 & 20.2 & 20.4 & $21: 8$ \\
\hline $\begin{array}{l}\text { East North } \\
\text { Central }\end{array}$ & $14: 6$ & 14.6 & 14.6 & $14.6^{\circ}$ & 14.6 & 14.6 & 14.6 & 14.7 & 14.7 & 15.2 \\
\hline $\begin{array}{l}\text { West North } \\
\text { Central }\end{array}$ & - 8.2 & 8.2 & 8.2 & 8.2 & 8.2 & $\cdot 8.2$ & 8.2 & 8.3 & 8.3 & 8.6 \\
\hline South Atlantic & 84.6 & 84.6 & 84.9 & 86.9 & 87.9. & 88.2 & 88.5 & 88.8 & $\begin{array}{l}-\quad 88.9 \\
\end{array}$ & 89.5 \\
\hline $\begin{array}{l}\text { East South } \\
\text { Central }\end{array}$ & - $\quad 32.6$ & 32.6 & 32.6 & 37.0 & 37.8 & 38.6 & 39.9 & 40.0 & 40.0 & 40.1 \\
\hline $\begin{array}{l}\text { West South } \\
\text { Central }\end{array}$ & 26.2 & 26.2 & 26.2 & 30.2 & .30 .9 & 31.6 & 32.8 & 32.8 & 32.8 & 32.8 \\
\hline Mountain & 12.5 & 12.5 & 12.5 & 12.5 & 12.5 & 12.5 & 12.7 & 12.7 & 13.5 & 13.5 \\
\hline Pacific & 42.2 & 42.2 & 42.2 & 42.2 & 42.5 & 43.1 & 64.6 & 66.3 & 68.6 & 68.9 \\
\hline Total & 259.5 & 259.5 & .259 .8 & 270.2 & 273.0 & 275.3 & 299.8 & 303.3 & 306.8 & 311.5 \\
\hline
\end{tabular}


The quantities are mapped from the U.S.F.S. regions to census divisions using the same matrix as for whole tree chips.

\section{Mill Residues}

Data on mill residue quantities are available from Forest Statistics of the. United States, 1987 (Waddell, et al., 1989) and the USDA/FS (1990) publication. Mill residues are reported by the following wood categories: coarse wood, fine wood, and bark residues for 8 regions and 4 uses. The 4 mill residue uses are: fiber, fuel, other, and not used. The mill residue use categories fuel and not used are considered. Fiber and other are used in designated paper and pulp industries and therefore not considered as available wood resourse. It is arbitrarily assumed that 50 percent of the category not used is an available, wood resource for all sectors.

For the use category fuel, 36 percent of coarse wood and 10 percent of fine and bark residues are available. The 10 percent availability of fine and bark residues represents the market share to noncaptive markets while the 36 percent availability of coarse wood is based on estimates-by Skog (personal.communication to Anthony Turhollow, November 24, 1994). Coarse wood is mainly used in the residential sector while fine and bark residues are consumed in the industrial, commercial, and electricity generating sector.

Prices for residues at the mill were obtained from Skog (personal communication to Anthony Turhollow, November 24, 1992). A cost component for the transportation of wood of $\$ 0.187$ per million Btu is used, which is based on an assumed delivery distance of 20 miles using a cost of. \$0.14 per dry-ton (12 percent moisture) mile and 17 million Btu per dry ton.

Table 6A-5 shows the supply schedule for mill residues. 
Table 6A-5. Mill Residues Available for Energy Use in Noncaptive Markets

\begin{tabular}{|c|c|c|c|c|c|}
\hline \multirow[b]{2}{*}{ Census Region } & \multirow[b]{2}{*}{ Skog Region } & \multicolumn{2}{|c|}{ Quantity of Residues } & \multicolumn{2}{|c|}{ Prices of Residues } \\
\hline & & Coarse & Fine and Bark & Coarse & - Fine and Bark \\
\hline - & & \multicolumn{2}{|c|}{ Trillion Btu } & \multicolumn{2}{|c|}{ 1987\$Million Btu } \\
\hline New England & North & 1.47 & 0.79 & 3.263 & 0.930 \\
\hline Middle Atlantic & North & 1.52 & 0,82 & 3.263 & 0.930 \\
\hline East North Central & North & 4.09 & 4.40 & 3.263 & 0.930 \\
\hline West North Central & North & 2.51 & 2.82 & 3.263 & 0.930 \\
\hline Sonth Atlantic & South & 9.48 & 15.84 & 3.060 & 0.888 \\
\hline East South Central & South & 1.70 & 9.89 & 3.060 & 0.888 \\
\hline West South Central & Sonth & 1.32 & 7.68 & 3.060 & 0.888 \\
\hline Mountain. & West & 1.63 & 6.40 & 2.793 & 0.863 \\
\hline Pacific & West & 17.75 & 13.41 & 2.793 & 0.863 \\
\hline Total & & 41.48 & 62.05 & & \\
\hline
\end{tabular}

\section{Other Wood}

Other wood includes wood waste generated by the secondary forest products industry (e.g., furniture), construction debris, waste pallets, and demolition wastes and is a relatively small quantity in comparison to other sources of wood.

The quantities and prices for other wood are obtained from Skog (personal communication to Anthony Turhollow, December 1, 1992). Skog provides data for three divisions of the United States. The data were mapped from the three regions to census regions using 1990 population data. The total amount of other wood, 5.285 trillion Btu, is quite sinall in comparison to whole tree chipping or logging residues. The quantities of other wood available by census deivision are presented in Table 6A-6. The price in all census divisions is $\$ 20.71$ per million Btu. 
Table 6A-6. "Other Wood" Supplies by.Census Division

\begin{tabular}{||l|c||l|c|}
\hline Census Division & Trillion Btu & Census Division $: \because$ & Trillion Btu \\
\hline New England & 0.274 & East South Central & 0.211 \\
\hline Middle Atlantic & 0.779 & West South Central & 0.371 \\
\hline East North Central & 0.870 & Mountain & 0.469 \\
\hline West North Central & 0.366 & Pacific & 1.287 \\
\hline South Atlantic & 0.658 & Total & 5.285 \\
\hline
\end{tabular}

SOURCES: Oak Ridge National Laboratory, "Data and Sources Biomass Supply", Draft " prepared for EIA under Contract No. DE-AC05-84OR21400; Oak Ridge, TN, June 27, 1993.

McQuillan, A.; Skog,K.; Nagle, T.; Loveless, R.. "Marginal cost supply curves for utilizing forest waste wood in the United States", unpublished manuscript, 1984.

U.S. Department of Agriculture, Forest Service, "An analysis of the timber situation in the United States: 1989-2040", General Technical Report RM-199, Rocky Mountain Forest and Range Experiment Station, Fort Collins, CO, 1990.

U.S. Department of Agriculture, Forest Service, "The Forest Biomass Resource in the United States", General Technical Report WO-57, Washington, D.C., 1990.

Waddell, K. L., D.D. Oswald, and D.S. Powell, "Forest Statistics of the. United States, 1987", Resource Bulletin PNW-RB-168; U.S. Department of Agriculture, Forest Service, Pacific Northwest Research Station, Portland,. OR, 1989.

\section{MODEL INPUT: WCABMEL ,}

DEFINITION: Available generating capacity [MW] in NERC region $R N$ and year $T$.

The maximal generating capcity is determined by the maximal value in each regional supply curve and converted into $M W$ using the performance characteristics of the wood technology, represented in the RFM.

SOURCES: Craig, K.R.; Mann, M.K.. 1993. Cost and Performance Analysis of Integrated Gasification Combined Cycle (IGCC) Power Systems Incorporating a Directly Heated Biomass Gasifier. Milestone Completion Report. NREL. December 1993. 


\section{MODEL INPUT: - WCCBMEL}

DEFINITION: $\quad$ Capital costs for electricity sector in Census division $R$ in year $T$

WCCBMEL represents the capital cost for an advanced Wood Integrated Gasification Combined Cycle (IGCC) technology which is estimated to be commercially available in the year 2000 . The cost estimates are based on a detailed analysis and performed by NREL.

SOURCES: Craig, K.R.; Mann, M.K.. 1993. Cost and Performance Analysis of Integrated Gasification Combined Cycle (IGCC) Power Systems Incorporating a Directly Heated Biomass Gasifier. Milestone Completion Report. NREL. December 1993.

\section{MODEL INPUT: WVCBMEL}

DEFINITION: Variable costs for wood electricity generation for the utility sector in NERC region $R N$ in year $T$

Variable cost is model determined: It is a composite of two factors: 1) a constant factor accounting for operational maintenance expenses and 2) fuel cost. Since there is no vehicle to pass fuel cost to the ECP, the cost for wood is converted into mills per $\mathrm{kWh}$ and added as an. additional variable O\&M cost component. The constant factor is $8.94 \mathrm{mills} / \mathrm{kWh}$.

SOURCE: Electric Power Research Institute, "Technical Assessment Guide", Vol. 1, Revision 7, EPRI TR-102276S, Palo Alto, CA, June-1993.

\section{MODEL INPUT: WOCBMEL}

DEFINITION: Fixed O\&M costs for wood technology in NERC region RN and year T.

The fixed O\&M cost are $84.69[1987 \$ / \mathrm{kW}]$ according to the NREL source. It is assumed to be constant across all regions and for all years.

SOURCE: Craig, K.R.; Mann, M.K.. 1993. Cost and Performance Analysis of 'Integrated Gasification Combined Cycle (IGCC) Power Systems Incorporating a Directly Heated Biomass Gasifier. Milestone Completion. Report. NREL. December 1993.

\section{MODEL INPUT: WCFBMEL}

DEFINITION: Capacity factor for the utility sector in NERC region RN in year $T$

Capacity factor is assumed to be constant for all years and all regions at a value of 0.8 . 
SOURCE: Craig, K.R.; Mann, M.K.. 1993. Cost and Performance Analysis of Integrated Gasification Combined Cycle (IGCC) Power Systems Incorporating a Directly Heated Biomass Gasifier. Milestone Completion Report. NREL. December 1993.

\section{MODEL INPUT: WHRBMEL}

\section{DEFINITION: $\quad$ Heat rate for wood technology in NERC region RN in year $T$}

The heat rate in the wood submodule represents a composite of two technolgies: 1) the existing direct fired or co-fired wood boilers and 2) the future advanced wood integrated gasification combined cycle (IGCC) technology estimated to be commercially available by the year 2000 . Therefore the heat rate must reflect the transition from the existing and current wood technology to the advanced IGCC technology beginning with the year 2000. An early NEMS projection was used to calcualte capacity-weighted average heat rates. By the year 2010 a technology mix of 52 percent current and 48 percent of future technology is attained.

The heat rates for the individual wood technologies and those representing the technology mix are listed in Table 6. -7.

Table 6A-7. Heat Rate for Represented Wood Technologies

\begin{tabular}{||c|c|c|c||}
\hline Year & Direct Fired Boiler & IGCC & Weighted Mix \\
\hline & [BTU/kWh] & [BTU/kWh] & [BTU/kWh] \\
\hline 1990 & 13762 & 11045 & 13762 \\
\hline 1991 & 13363 & 11023 & 13363 \\
\hline 1992 & 12964 & 11002 & 12964 \\
\hline 1993 & 12566 & 10981 & 12566 \\
\hline 1994 & 12167 & 10960 & 12167 \\
\hline 1995 & 11768 & 10939 & 11768 \\
\hline 1996 & 11768 & 10753 & 11768 \\
\hline 1997 & 11768 & 10567 & 11768 \\
\hline 1998 & 11768 & 10380 & 11768 \\
\hline 1999 & 11768 & 10194 & 11768 \\
\hline 2000 & 11768 & 10008 & 11745 \\
\hline 2001 & 11768 & 10008 & 11684 \\
\hline 2002 & 11768 & 10008 & 11623 \\
\hline 2003 & 11768 & 10008 & 11623 \\
\hline 2004 & 11768 & 10008 & 11623 \\
\hline 2005 & 11768 & 10008 & 11623 \\
\hline 2006 & 11768 & 9803 & 11606. \\
\hline 2007 & 11768 & 9597 & 11037 \\
\hline 2008 & 11768 & 9392 & 10935 \\
\hline 2009 & 111768 & 9186 & 10853 \\
\hline 2010 & 11768 & 8981 & 10426 \\
\hline & & & \\
\hline
\end{tabular}


SOURCE: Biomass Power Gasification System, Technology Characterization, DOE, Office of Energy Efficiency and Renewable Energy, 3/17/1994. 
$\therefore$ 


\section{Appendix 6-B: Mathematical Description}

The following equation calculates the total energy consumption from all sectors and all regions in trillion Btu. It is assumed that 3 percent of the industrial consumption is in the noncaptive market.

$$
Q U C I=Q B M E L_{R, T I}+Q B M C M_{R, T I}+0.03 Q B M I N_{R, T I}
$$

where:

$$
\begin{aligned}
& \text { QUCI = Quantity of wood energy consumed in all sectors (trillion Btu); } \\
& \dot{Q} B M E L_{R, T l}=\text { Quantity of wood consumed by utilities in Census division } R \text { in } \\
& \text { year Tl; } \\
& Q B M C M_{R, T I}=\cdot \quad \text { Quantity of wood consumed in the commercial sector in Census } \\
& \text { division } R \text { in year } T 1 \text {; and } \\
& Q B \dot{M I N} N_{R, T I}=\text { Quantity of wood consumed in the industrial sector in Census } \\
& \text { division } R \text { in year } T \text {. }
\end{aligned}
$$

- The Wood Submodule uses a scheme to do a linear interpolation between two steps on the supply curve to determine the price of wood PUCI given a quantity of $Q U C I$. The interpolation is expressed as:

$$
P U C I=P R W T_{I}+\left[\frac{Q U C I-Q W T_{R, I}}{Q W T_{R, I+1}-Q W T_{R, I}}\right] x\left(P R W T_{I+1}-P R W T_{I}\right)
$$

where:

$$
\begin{aligned}
& P U C I=\text { Price of wood for all sectors, in dollars per million Btu; } \\
& P R W T_{I}=\text { Price from the all-sector supply schedule for supply function step } \\
& Q U C I \\
& Q W T_{R, I}=\quad \begin{array}{l}
\text { Quantity of wood consumed in all sectors in trillion Btu; and } \\
\text { Qupply function step } I \text { in Census division } R .
\end{array}
\end{aligned}
$$


For the commercial, industrial, and utility sectors, the sector supply prices $P B M C M_{R, T I}$, $P B M T N_{R, T l}$, and $P B M E L_{\mathrm{R}, T 1}$, are assigned the value of $P U C I$ in Equation (6B-2).

The price PUCI of wood is computed for each Census region. To apply $P U C I$ to NERC regions a simple mapping approach is used. The lowest price of a Census region that overlaps with a given NERC region establishes the price in this NERC region. This approach is based on the assumption that the lowest price quantities of wood are consumed exclusively rather than the higher priced commodity. Mathematically, it is expressed as:

$$
P W N R_{R N}=\min \left[P U C I_{R}\right] \cdot \text { for all } \operatorname{CDTONR}_{R N_{,},} \neq 0
$$

where:

$$
\begin{array}{ll}
P U C I= & \begin{array}{l}
\text { Price of wood for all sectors, in dollars per million Btu evaluated } \\
\text { for all Census regions } R .
\end{array} \\
P W N R_{R N}= & \begin{array}{l}
\text { Price of wood for all sectors, in dollars per million Btu in NERC } \\
\text { region } R N .
\end{array} \\
C D T O N R_{R N, R}= & \text { mapping matrix to map Census regions into NERC regions. }
\end{array}
$$

Since the wood submodule does not have a vehicle to pass fuel cost to the ECP module; the price $P W N R$ of wood is converted into a variable O\&M cost component and added to the constant variable cost factor. The conversion is expressed as:

$$
W V C B M E L_{R N, T}=W V C_{R N, T}+P \mathscr{P W R} R_{R N, T} \cdot W H R B M E L_{R N, T} \cdot C_{1}
$$

where:

$$
\begin{aligned}
& \mathrm{WVC}_{R N, T} . \quad=\text { Constant variable O\&M cost component }(8.94[\mathrm{mills} / \mathrm{kWh}]) \\
& \text { in NERC region } R N \text { and year } T \text {. - } \\
& \text { WHRBMEL }_{R N, \dot{T}} \quad=\quad \begin{array}{l}
\text { Heat rate for wood technology in NERC region } R N \text { and } \\
\text { year } T .
\end{array} \\
& C_{1}=\text { conversion factor to transform from } \$ / M M B T U * \\
& \mathrm{BTU} / \mathrm{hWh} \text { to mills } / \mathrm{kWh}
\end{aligned}
$$




\section{Appendix 6-C: Bibliography}

Associates in Rural Development. Impact of Large Biomass Demand Centers on the Forest Resource Base. Report prepared for the Northeast Regional Biomass Program by Associates in Rural Development, Burlington, VT. National Technical Information Service, Springfield, VA. 1986.

Draper, H.M., R.T. Brooks, Jr., J.D. Thomas, and L.A. Weaver. The Biomass Energy Potential of Mississippi. Tennessee Valley Authority, Norris, TN and Mississippi Forestry Association, Jackson, MS. 1989.

E.C. Jordan Co. and Maine Audubon Society. Wood Chips: An Exploration of Problems and Opportunities. Report prepared for the Northeast Regional Biomass Program by E. C. Jordan Co., Portland, ME and Maine Audubon Society, Falmouth, ME. National Technical Information Service, Springfield, VA. 1985.

Ellis, B.K. and J.A. Brown. Production and Use of Industrial Wood and Bark Residues in the Tennessee Valley. TVA/ONRED/LER--86/1. Tennessee Valley Authority, Norris, TN. 1986.

English, Burton C. and Sean Coady. The costs of producing biomass in the United States for selected land resource regions. Unpublished document, 1990.

Klass, D.L. The U.S. Biofuels Industry. Paper presented at the International Renewable Energy Conference, Honolulu, Hawaii, September 18-24, 1988.

Larsen, E.D. and R.H. Williams. Biomass-Fired Steam-Injected Gas Turbine Cogeneration. Biologue 6(5):12-19, 1989/1990.

McQuillan, A., K. Skog, T. Nagle, and R. Loveless. Marginal Cost Supply Curves for Utilizing Forest Waste Wood in the United States. Unpublished manuscript, 1984. .

Ostlie, L.D. The Whole Tree Burnèr: A New Technology in Power Generation. Energy Performance Systems, Inc., Minneapolis, MN, 1989.

Rice, D. Presentation at EPRI-Sponsored Biomass Production Economics Workshop. Nashville, TN, October 22-23, 1992.

Skog, K. Presentation at EPRI-Sponsored Biomass Production Economics Workshop. Nashville, TN, October 22-23, 1992.

Turnbull, J. Presentation at EPRI-Sponsored Biomass Production Economics Workshop. Nashville, TN, October 22-23, 1992. 
Trumble, D.A. Wood Energy II: Forecasts of Regional Sectoral Wood Energy Consumption (1990-2010). Draft report submitted to Energy Information -Administration, U.S. Department of Energy, October. Prepared by Oak Ridge National Laboratory, Oak Ridge, TN. 1991 .

Turhollow, A.F. et al. "Data and Sources, Biomass Supply." Draft report prepared for the Energy Inforamtion Administration, U.S. Department of Energy, by Oak Ridge National Laboratory. Oak Ridge, TN. July 1993.

U.S. Environmental Protection Agency. AIRS Facility'Subsystem: Source Classification Codés and Emission Factor Listing for Criteria Air Pollutants, Technical Support Division, Office of Air Quality Planning \& Standards, Research Triangle Park, North Carolina. March 1990.

USDA/ERS. Economic indicators of the farm sector: Costs of production--Major field crops, 1989. ECIFS 9-5. Agriculture and Rural Economy Division, Economic Research Service, U.S. Department of Agriculture, 1991.

USDA/FS. An Analysis of the Timber Situation in the United States: 1989-2040. A Technical Document Supporting the 1989 USDA Forest Service RPA Assessment. General Technical Report RM-199. Rocky Mountain Forest and Range Experiment Station, Forest Service, U.S. Department of Agriculture, Fort Collins, CO. 1990.

Waddell, K.L., D.D. Oswald, and D.S. Powell, Forest Statistics of the United States, 1987; Resource Bulletin PNW-RB-168, United States Department of Agriculture Forest Service, Pacific Northwest Research Station, Portland, OR. 1989. 


\section{Appendix 6-D: Model Abstract}

Model Name:

Wood Submodule.

Model Acronym:

None.

\section{Description:}

Most Recent Model Update:

November 1994.

Part of Another Model?:

The Wood Submodule is a component of the Renewable Fuels Module (RFM) of the National Energy Modeling System (NEMS).

Official Model Representative:

Roger Diedrich.

Documentation:

Archive Media and Installation Manual(s):

Energy System Described:

Non-captive wood supply and associated price.

Coverage:

USA.

Modeling Features:

Nine seperate regions.

Non-DOE Input Sources:

Computing Environment:

Indépendent Expert Reviews Conducted:

None.

Status of Evaluation Efforts by Sponsor: 


\section{1}




\section{Appendix 6-E: Data Quality and Estimation Processes}

\section{Derivation of the All-Sector Wood Supply Curve Logging Residue and Whole Tree Data}

\section{Data Sources and Methodology}

Data for logging residue and whole tree supplies and prices were obtained from an unpublished manuscript entitled "Marginal Cost Supply Curves for Utilizing Forest Waste Wood in the United States", written for the United States Forest Service. The document was authored by McQuillan, Skog, Nagle, and Loveless, and was completed in 1984. The purpose of the study was to determine supply conditions for making use of "waste" wood, or that wood which is currently "unmerchantable", in each of the nine Forest Service Regions: This was done separately for hardwoods and softwoods.

Using 1976 inventory data and U.S.F.S. projections for 1990, 2010 and 2030, McQuillan, et al. estimated the available inventories of four different categories of waste wood: logging residues; rough, rotten, and salvable dead trees; excess sapling trees; and excess small pole.trees. The major source of this information was the USDA Forest Service Resource Report No. 23, "An Analysis of the Timber Situation in the United States, 1952-2030" (1982). In order to estimate a single supply curve for each of the regions, the authors assumed that each of the four different. waste wood types would be tranisformed into a single, homogeneous product: inbarked wood chips produced at the mill site.

For energy production purposes, wood chips can easily be treated as a homogeneous product. However, in the attempt to portray supply conditions as accurately as possible, it was decided to treat logging residues and whole tree supplies separately in the Wood Submodule. Supply conditions differ for these two waste wood types, as the supply of logging residues is mostly a function of logging operations, while the supply of whole trees depends largely on growing conditions. For the latter category, it was necessary to divide the McQuillan inventory estimates by twenty in order to approximate a sustainable harvest of whole chipping trees.

For each of the 18 "situations" (region and wood type, hard or soft), the McQuillan team divided - the waste wood categories into four haul distance classes and three "slope/operability" classes, producing 156 cost strata. Costs for removal, transport and chipping of waste wood were based on data for U.S.F.S. Region 1, which were obtained from Richard R. Withycombe (1982), "Estimating Costs of Collecting and Transporting Forest Residues in the Northern Rocky Mountain Region." These extraction and haul costs were mapped to Regions 2 through 7 using data from "Report 5 of the Summary of Reports of Timber Sale Type, Stand Size, Site and Ownership". (1977, Fort Collins, Colorado); to Region 8 using data from Adams and Haynes (1980), "The 1980 Softwood Timber Assessment Market Model: Structure, Projections and Policy. Simulations" and Plummer (1977), "Harvesting Cost Analysis, In: Logging Cost and 
'Production Analysis"; and to Region 9 using data from the Forest Service Handbook \#2409-22, . "Timber Appraisal Handbook".

Slope/operability classes include "feller/buncher" (representing 0-20 percent on-site slope), "crawler tractor" (20-40 percent slope); and "cable yard" (40+ percent slope). As haul distance (to the nearest mill) and slope increase, the cost of salvaging the wood increases rapidly. Accessibility by road is another major cost consideration; however, the authors assumed that a certain percentage of the resource was accessible on existing roads, and that new roads would not be built solely to access a source of waste wood. Ultimately, the wood classes described above were aggregated into discrete regional supply schedules and then combined to form a national total for both hardwoods and softwoods:

\section{Data and Model Limitations}

The authors noted that aggregating cost and availability conditions which can vary greatly among. the thousands of potential sites to the national and even regional level is fraught with difficulties. These are compounded -when projections of supply conditions are made over a number of years. Waste wood is defined as wood which is not currently "merchantable", that is, wood which is currently unutilized. The quantity of standing trees and logging residues that fall under this category in the future will depend on market conditions. As log prices increase and the availability of high-quality, old-growth timber decreases, minimum tree. size and top size parameters will' shrink, and second- and third-growth stands will be managed more intensively to maximize yields and make up for the loss of old-growth timber. These factors will serve to reduce the number of saplings, pole trees, and rough, rotten, and salvable dead trees available for chipping.

The most sensitive assumptions are those associated with removal and hauling costs. Some sensitivity analysis was performed for the "North Rocky Mountain" region, and this indicated that costs were very. sensitive to changes in the haul distance and slope/operability parameters. Since the elasticities are very high, inaccuracies in the original data on which location and operability classifications were based could have a major effect on potential supply prices.

In addition to location and availability, the supply prices also depend heavily on technology assumptions. Improvements in on-site operating techniques could greatly increase the quantity of waste wood available for economic use, and improvements in chip-hauling technology could obviate the need for removal of waste wood to mills for chipping, further reducing production. costs. Other equipment innovations are expected to occur within the next few decades, reducing costs and increasing the availability of salvageable waste wood. .

Finally, the authors mentioned demand factors that may lead to a change in market conditions. For example, if pulp and paper production increases in importance relative to lumber and plywood, the supply of waste wood available for other uses may be reduced. Debarking, which is necessary for the manufacture of some products, is quite expensive and changes in the demand for those products could also have an effect on the waste wood supply conditions. 
Table 6E-1. Aggregated Supply Schedule for Whole Tree Chips, Logging Residues, Mill Residues, and "Other Wood"

\begin{tabular}{|c|c|c|c|c|c|c|c|c|c|}
\hline & \multicolumn{9}{|c|}{ Quantity Supplied (Trillion Btu) } \\
\hline $\begin{array}{l}\text { Price } \\
\text { (\$1987/ } \\
\text { MMBtu) }\end{array}$ & Northeast & Southeast & $\begin{array}{l}\text { North } \\
\text { Central }\end{array}$ & $\begin{array}{l}\text { South } \\
\text { Central }\end{array}$ & $\begin{array}{c}\text { North } \\
\text { Rocky } \\
\text { Mountain }\end{array}$ & $\begin{array}{c}\text { South } \\
\text { Rocky } \\
\text { Mountain }\end{array}$ & $\begin{array}{c}\text { Pacific } \\
\text { Northwest } \\
\text { East }\end{array}$ & $\begin{array}{l}\text { Pacific } \\
\text { Northwest } \\
\text { West }\end{array}$ & $\begin{array}{l}\text { Pacific } \\
\text { Sonthwest }\end{array}$ \\
\hline $0.474^{\prime}$ & $0.0^{\circ}$ & 0.0 & 0.0 & 0.0 & 0.0 & 0.0 & 0.0 & 0.0 & 0.0 \\
\hline 0.542 & 0.0 . & 0.0 & 0.0 & 0.0 & 0.0 & 0.0 & 0.0 & 0.0 & 0.0 \\
\hline 0.711 & 0.0 & 0.0 & 0.0 & 0.0 & 0.0 & 0.0 & 0.0 & 0.0 & 0.0 \\
\hline 0.813 & 0.0 & 0.0 & 0.0 & 0.0 & 0.0 & 0.0 & 0.0 & 0.0 & 0.0 \\
\hline 0.948 & 0.0 & 0.0 & 0.0 & 0.0 & 0.0 & 0.0 & 0.0 & 0.0 & 0.0 . \\
\hline 1.083 & 0.0 & 0.0 & 0.2 & 0.1 . & 0.0 & 0.0 & 0.0 & 0.0 & 0.0 \\
\hline 1.185 & 0.0 & 0.0 & 0.2 & 0.1 & $67 . \dot{4}$ & 9.1 & 8.1 & 0.2 & 0.0 \\
\hline 1.354 & 0.0 & 0.0 & 0.2 & 0.1 & 77.6 & 9.7 & 8.6 & 1.6 & 6.9 \\
\hline 1.422 & 3.0 & .3 .1 & 7.2 & 4.0 & 240.4 & 47.4 & 40.9 & 5.0 & 8.6 \\
\hline 1.625 & 5.9 & 6.1 & 8.5 & 4.8 & 260.2 & $53.8^{\circ}$ & 46.4 & 14.2 & 42.3 \\
\hline 1.659 & 15.9 & 16.4 & 31.2 & 17.2 & 352.7 & .129 .7 & 109.7 & 21.2 & 50.1 \\
\hline 1.896 & 62.9 & 64.9 & 89.1 & 49.3 & 412.6 & 211.7 & 171.3. & 60.2 & 110.1 \\
\hline 2.133 & $99.9^{\circ}$ & 103.7 & 144.2 & 80.1 & 455.9 & 248.7 & 193.9 & 72.2 & 153.3 \\
\hline 2.167 & 105.8 & 109.8 & 146.4 & 81.7 & 459.2 & 251.7 & 196.1 & 93.4 & 187.8 \\
\hline 2.370 & 111.7 & 115.8 & 147.6 & 82.3 & 465.8 & 256.9 & 200.5 & 94.6 & 195.9 \\
\hline 2.438 & 117.8 & 122.1 & 148.4 & 83.3 & 468.9 & 257.8 & 201.1 & 120.8 & 209.2 \\
\hline 2.709 & 120.6 & 125.0 & 149.0. & 83.8 & 470.7 & 259.0 & 202.1 & 127.7 & 220.4 \\
\hline $2.844^{\prime} \quad \cdot$ & 130.9 & 135.7 & 157.3 & 88.4 & 672.4 & 312.3 & 248.1 & 132.8 & 228.3 \\
\hline 3.250 & 131.4 & 136.2 & $157.5^{\circ}$ & . $\quad 88.5$ & 794.9 & 339.0 & 2720 & 139.6 & 293.3 \\
\hline 3.318 & 136.9 & 141.9 & 160.7 & 90.3 & 1280.3 & 587.1 & 493.2 & 143.5 & 309.1 \\
\hline 3.792 & 162.1 & 167.9 & 185.5 & 104.2 & 1395.3 & $\begin{array}{r}\cdot \quad 743.0 \\
\end{array}$ & 627.6 & 204.3 & 453.9 \\
\hline 4.266 & 239.1 & 247.4 & 362.5 & 201.2 & .1436 .1 & 787.8 & 631.7 & 234.2 & 463.4 \\
\hline 4.334 & 273.6 & 282.9 & 402.6 & 223.7 & 1453.8 & 802.7 & 636.9 & 280.8 & 489.5 \\
\hline 4.740 & 299.9 & 310.1 & 467.1 & 259.0 & 1466.8 & 818.2 & 637.7 & 282.3 & 498.8 \\
\hline 4.876 & 324.5 & .335 .5 & 489.7 & 2727 & 1478.9 & 824.7 & 638.9 & 342.6 & 553.8 \\
\hline 5.214 & 327.3 & 338.4 & 489.7 & 2729 & 1480.3 & 824.8 & 638.9 & 343.9 & 558.8 \\
\hline 5.417 & 328.1 & 339.2 & 489.8 & 273.1 & 1480.6 & 824.8 & $638.9^{\circ}$ & 355.5 & 563.1 \\
\hline 5.688 & 329.6 & 340.7 & 490.4 & 273.4 & .1481 .3 & 824.9 & 638.9 & 355.7 & 563.4 \\
\hline
\end{tabular}




\section{Geothermal Electric Submodule}

\section{Model Purpose}

The purpose of the Geothermal Electricity Submodule is to model the current and future regional supply, capital cost, and operation and maintenance costs of electric generating facilities exploiting U.S. hydrothermal resources, based on available resource data and on current technology with reasonable assumptions as to leaming-curve and technological improvements.

More specifically, in the context of NEMS, the purpose of GES is:

- to provide the Electricity Market Module's (EMM's) capacity planning submodule with the amount of new capacity that can be built, with related cost and performance data;

- to provide EMM's electricity dispatch submodule with cost and performance data for all installed capacity, including capacity reported on EIA surveys as existing or planned and capacity added as a result of a model calculation - known as "unplanned" capacity, or more accurately "modeled" capacity.

\section{Relationship of the Geothermal Electricity Submodule to Other Models}

The major relationships between GES and other NEMS components are:

- GES provides new capacity availability, performance and cost information for the Electricity Capacity Planning (ECP) submodule's use in making its planning decisions;

- GES provides cost and performance data on installed capacity for use by the Electric Fuel Dispatch (EFD) submodule; .

- GES uses ECP new capacity build decisions obtained from the EMM output common block, EMMOUT;

- As an option, GES uses drilling and other field cost improvement parameters used in the Oil and Gas Supply module (OGSM);

- GES uses the following EMM data:

- financial parameters and tax data for calculations related to the competing 'geothermal resource sites

- ECP's "cut-off" or "shadow" price to truncate the geothermal supply curves. 


\section{Model Rationale}

The GES projects regional supply curves relating new geothermal electric capacity and the capital cost to provide the new capacity. Annual operations and maintenance (O\&M) costs are also projected for the new capacity. These are used to determine the costs for the amount of new geothermal capacity requested by the market, repreșented in NEMS by the Electricity Market Module (EMM). Costs for previously installed capacity and those for new capacity are consolidated to project the costs for all geothermal electricity dispatched by the market.

The supply curves are aggregated based on regions defined by the North American .Electric Reliability Council (NERC). Supply curves are modeled for NERC regions 11, 12, 13, and 15, the only regions of the U.S. with economic geothermal resources.

The cost of generating electricity from geothermal resources is.largely a function of the resource temperature, depth, and chemistry. Because these parameters, and consequently the cost of electricity, vary considerably at different resource sites, the methodology employs pre-processing site-specific geothermal resource data to model site-specific costs and operational characteristics. The data pre-processing is performed by the PC-based GES Data Preprocessor (GESDPP), which was adapted from an existing PC-based model, IM-GEO. ${ }^{18}$ Developed by Sandia National Laboratory in the mid 1980s, "IM-GEO" stands for Impacts of Research and Development on the Cost and Performance of Geothermal Electric Systems.

The resource data set includes data on 51 known geothermal resource sites in the U.S. The site data include measured parameters as well as parameters which were estimated based on other measured data for the site in question or on more complete knowledge of a geologically and hydrologically similar site. These data were compiled during a study completed in 1991 for EIA and the Geothermal Division of DOE ${ }^{19}$, using U.S. Geological Survey data and other sources.' The data include potential site capacity; reservoir parameters such as temperature, depth, salinity, noncondensible gas content; well costs; well mechanical life; time between well wộkovers; flow rate per well; etc.

The GESDPP, for each site, calculates the costs and performance of a single 50.MW (net) plant based on the site data and writes the output to a file which serves as input for GES. The costs are modeled on historical cost data for independent geothermal power projects developed during the mid to late 1980 s.

Using the output from the GESDPP, the GES models the incremental development of each resource site within the framework of a behavioral model based upon observed past experience with geothermal development. The key decision variable is the percentage of total site capacity previously installed. .

\footnotetext{
${ }^{18}$ Entigh, D., Livesay, B. and Petty, S., Geothermal Cost of Power Model IM-GEO Version 3.05: User's Manual, February 1989.

${ }^{19}$ Petty, Susan, et al, Supply of Geothermal Power from Hydrothermal Sources: A Study of the Cost of Power in 20 and 40 Years, Susan Petty. Consulting, Solana Beach, CA, June 1991.
} 
At previously undeveloped sites extreme conservatism dictates limiting initial development to a single plant of no more than $20 \mathrm{MW}$. After the initial plant has been installed, a larger plant may - be installed, but still no more than one $50 \mathrm{MW}$ plant at a time, until at least 15 percent of the estimated potential capacity has been successfully installed. Additional new capacity will not be considered during the lead time. Reflecting the greater confidence in the viability for further development after the first 15 percent of potential capacity has been installed, up to four $50 \mathrm{MW}$ plants may be built at the same time. Also, further capacity additions will be considered after the lead time of three years. As successive installations account for more and more of the estimated total potential capacity, conservatism returns in order to avoid overdevelopment of the site. The developer will not risk building more than $\mathbf{7 5}$ percent of the remaining undeveloped estimated site capacity.

\section{Fundamental Assumptions}

\section{Type of Resource}

Development of hydrothermal resources is the only commercially viable geothermal electric generation option with current technology, and therefore hydrothermal resources are the only geothermal resource considered in the GES. For the purpose of this study, a hydrothermal resource is defined as a large volume of hot water trapped in hot, permeable rock at depths up to 11,000 feet and with temperatures ranging from $220^{\circ} \mathrm{F}$ to $700^{\circ} \mathrm{F}$. The model is based upon the historical costs of exploring, confirming and developing hydrothermal resources and installing power plants to bring. incremental capacities on-line at known geothermal sites.

\section{Conversion Technologies}

Two types of geothermal technologies are modeled in the GES, dual-flash and binary cycle. These systems represent the complete cycle, from extraction of the hydrothermal fluids (equivalent to the fuel in convientional fossil-fueled systems) to electricity gerieration to reinjection of the spent geothermal fluid (often referred to as brine). Both technology types are used to convert liquid-dominated hydrothermal fluids into electricity. Technologies for dry steam reservoirs, such as the Geysers geothermal field in northern California, are not modeled since this type of resource is extremely rare and unrepresentative of the bulk of hydrothermal resources in the U.S. However, the existing capacity at the Geysers is included in total installed hydrothermal capacity in the GES for capacity dispatch.

Dual-flash plant technology is employed to convert the heat from high temperature (greater than $200^{\circ} \mathrm{C}$ ) liquid-dominated resources to electricity. With this technology, a portion of the geothermal liquid extracted from the reservoir is "flashed" to steam at two different pressures. The steam is separated and used to drive a conventional turbine-generator. The remaining liquid portion of the geothermal fluid is injected back into the ground. 
Electricity is generated from lower temperature (less than $200^{\circ} \mathrm{C}$ ) ${ }^{20}$ liquid-dominated resources using binary cycle technologies. The geothermal liquid is circulated through a closed-loop system where its heat is used to vaporize a secondary working fluid (hence the name binary) with a low boiling point, such as isopentane. The vapor of the secondary fluid is used to drive a turbinegenerator, and the cooled geothermal liquid is injected back into the-ground.

Based on the performance of existing geothermal power plants, a plant capacity factor of 80 percent is used. ${ }^{21}$

\section{Drilling and Extraction Technologies}

All geothermal electricity systems require drilling and extraction technologies derived from the petroleum industry. These "borrowed" technologies are modified to accommodate the high temperature and sometimes harsh chemical environments related to working with geothermal fluids. The GES models geothermal field development costs based on current geothermal drilling technology.

\section{Alternative Approaches}

U.S. geothermal resources were evaluated in the mid 1970 s by the U.S. Geological Survey ${ }^{22}$ (USGS) and in 1991 by Petty.et. al. (see footnote 1). These assessments included estimates of potential electric generation capacities in terms of number of megawatts for 30 years at known geothermal hydrothermal resource sites in the U.S. At the time of the USGS study, geothermal development in the U.S. was limited to The Geysers steam field in northern California, and exploration and delineation of the nation's liquid-dominated hydrothermal resources was only beginning. The 1991 study used the USGS study as a basis, but took advantage of considerable hydrothermal resource data and developmental experience gained in the interim.

The IM-GEO model developed by Sandia originally included only four generic geologic sites; typical of those found in the U.S. In 1992, it was modified to process data for all the sites identified in the 1991 Petty et. al. resource study. Thus, it made sense to incorporate it into the GES. However, since it was.coded in Quick Basic, it was decided to incorporate it as a PC-based data pre-processor, rather than rewrite it in Fortran as an endogenous component of GES. The data pre-processor is referred to as the GES Data Pre-Processor or GESDPP, for short. See Appendix 7-E for further details on the GESDPP.

\footnotetext{
${ }^{20} \mathrm{Petty}$; $\mathrm{Ct}$ al, (Ibid.) established $110^{\circ} \mathrm{C}$ as the minimum temperature for resource consideration (P. $\mathrm{G}$ ) and $200^{\circ} \mathrm{C}$ as the maximum temperature for the binary cycle technology (P. 11).

${ }_{12}^{21}$ EIA, Annual Energy Review, DOE/EIA 0384(93), Washington, DC, July 1994.

${ }^{22}$ Muffler, LP.J., editor, Assessment of Geothermal Resources of the United States - 1978: United States Geological Survey Circular 790, 1978.
} 
"Although various projections of future geothermal electric capacity have been made by numerous individuals and organizations over the last 30 years, these have been, for the most part, educated guesses. NEMS is the first national-level, energy supply and demand model incorporating a geothermal supply model which integrates geothermal resource assessments with a cost and performance model of geothermal electric generation systems.

\section{Geothermal Electricity Submodule Structure}

\section{Submodule Flow Diagrams}

This section 'contains two flow diagrams showing the logical structure of the Geothermal Electricity Submodule, its principal procedures, and the relationships among them.

Figure 6 provides a high level overview of the controlling procedure, Subroutine RFMGES. There are four sets of procedures at this level:

(1) Initialization Procedures - When RFMGES is first called (first iteration for the first simulation year), GEINIT reads data from files and calculates and sets other initial values. A flow diagram for GEINIT procedures is shown in Figure 7. Currently, a separate procedure GERDTMP reads from a file "shadow" prices used to truncate new capacity supply curves. It is separate because an interface is: to be established in the future whereby EMM will provide the values. Site costs and unplanned new capacity factors that are set initially through GEINIT are changed as the submodule is run for successive years. When "all year" looping is being used for the NEMS run, their initial values must be restored on subsequent calls to RFMGES for the first simulation year.

(2) Standard "Once-A-Year" Procedures - These procedures are characterized as "standard", because they comprise the principal algorithms of the model. Reflecting the dynamics of the interfaces with EMM, they generally are performed only once each year, on calls for the first iteration. However, they are performed on all calls for any NEMS runs with "all years" looping. As a second reflection of EMM interface dynamics, the procedures prodice output not for the current simulation year, but for the year following the current simulation year. As a special case, output is first produced for the current simulation year when it is the first simulation year, but later than 1990. Two procedures produce internal data used by the primary model procedures. GEGETFP obtains EMM tax rates and cost of capital parameters and calculates.nominal discount rates. GEUPSCO updates site costs, moving costs for the current simulation year from the "next year" to the "this year" position in the cost arrays and calculating the costs for the following year for the "next year" position. There are three principal model procedures. GECPDPR processes the new capacity build decisions made by the ECP, allocating the regional build amounts to individual sites in the region through the rew capacity supply curve data for the region. GEEDOPR updates installed capacity and related data for both sites and regional aggregations, and produces output for use by the EFD submodule of the EMM. GECPOPR uses a set of procedures to build new capacity supply curves and produce 
Figure 6. Geothermal Electricity Submodule Overview Flowchart

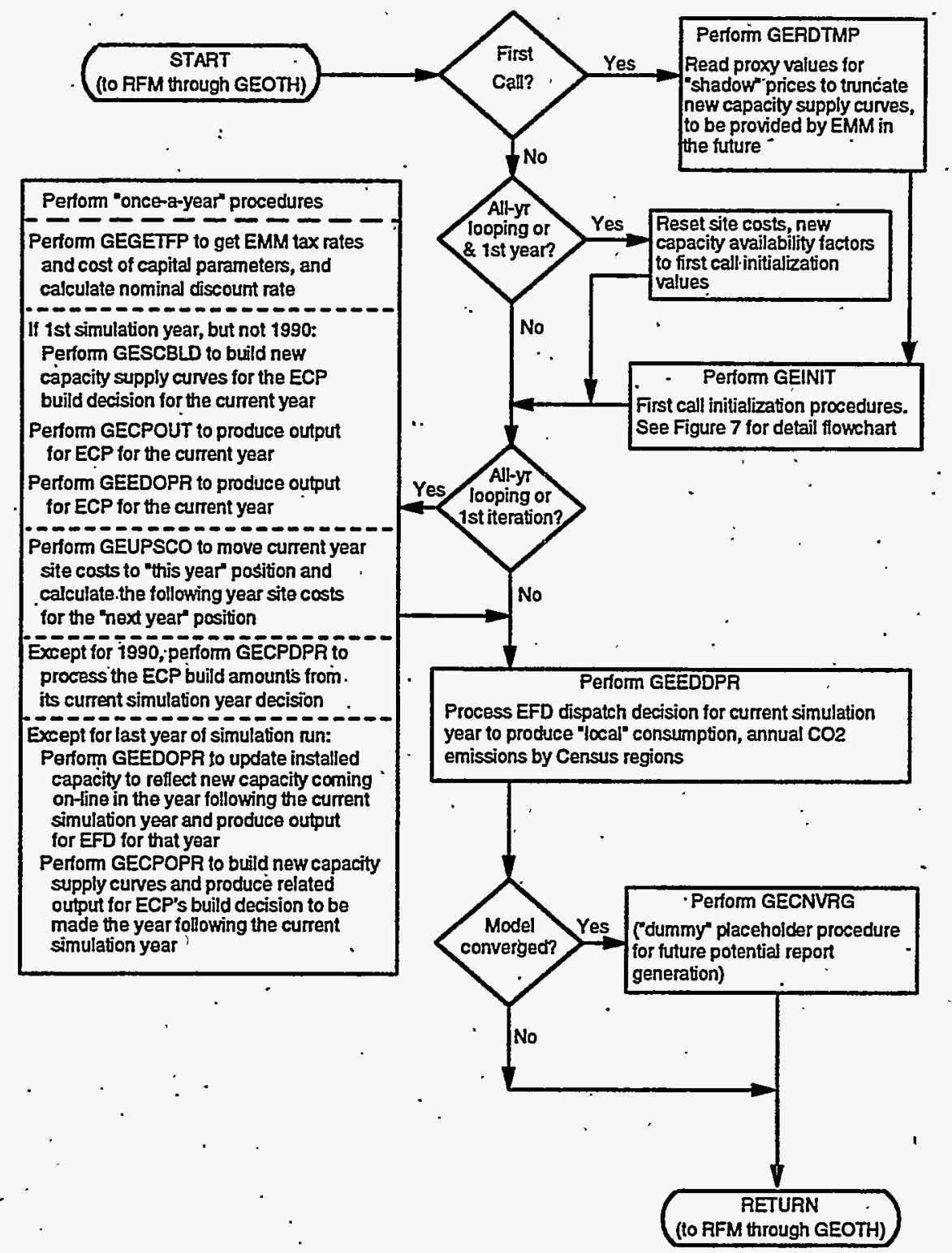

related output for use by the ECP submodule of the EMM. These procedures; GESCBLD and GECPOUT, respectively, are performed separately for the "first simulation year, but after 1990" special case.

(3) Dispatch Processing.Procedure - GEEDDPR is the only model procedure which is performed on all calls to RFMGES: Originally designed to produce for NEMS annual energy "consumption" and $\mathrm{CO}_{2}$ emissions by Census region from the dispatch amount by NERC region, the "output" is now local to RFMGES. It is available for reports for comparison with the NEMS output now being produced by the EMM. 
(4) "Converged" Procedure - GECNVRG is run only if a "post-convergence" reporting. run is signaled by the NEMS integrating module. Currently, this is a placeholder procedure with no functionality.

Figure 7 provides an overview of Subroutine GEINIT, the controlling procedure for first call initialization. GENNT, uses many' of the same lower level procedures used for "standard" processing. The procedures that are used to reset site costs and new capacity availability factors to their initial values on subsequent first simulation calls for "all year" looping runs are marked with an asterisk in Figure 7.

\section{Figure 7. GES First Call Initialization Overview Flowchart}

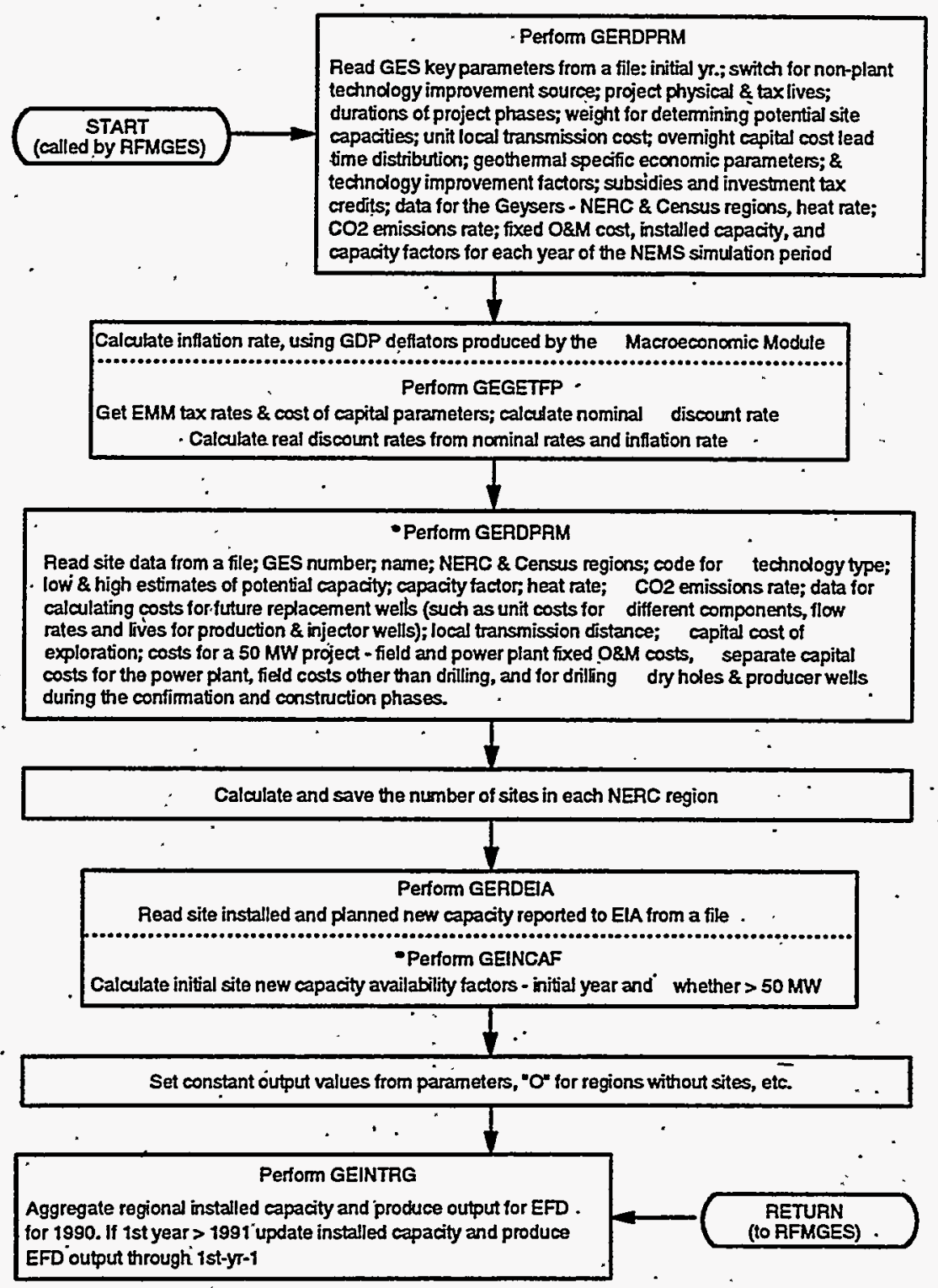




\section{Key Computations and Equations}

This section describes the most important equations of the GES model. All algorithms for each GES subroutine are mathematically described in full in Appendix 7-B. The section is divided into computations for individual sites and those for geographic regions. GES operations will generally fall into one of these levels or the other. However, the most important GES function of building new capacity supply curves entails first site computations, then regional computations.

\section{Computations for Geothermal sites}

Data for geothermal sites are read from a file by Subroutine GERDSITE into local variables with no site dimension. These variables have names beginning .with "GSF." Variables with computed site data or site data retained for use in computations have a site dimension and are included within the WGENUM Common block. Their names all begin with "WGES." In all site data computations outside of GERDSITE, "PS"' is the index or subscript used for the site dimension. Unless specified otherwise, all site computations are performed for each site, and this should be understood in the descriptions which follow.

New Capacity Availability Factors. WGESNCA $v_{P S, P Y}$ is the new capacity availability factor for site "PS" for year of availability "PY." Because the year definition relates to year of availability, the year dimension size is (MNUMYR+10), or 39. However, because only the first 26 positions of MNUMYR are.in use and an ECP planning horizon of 6 years, rather than 10, is in use, only the first $31(26+6-1)$ positions of the year dimension are used for WGESNCAv and all other GES variables with a (MNUMYR+10) year of availability dimension.

Values may be 0 (no new capacity available for the year), 1 (new capacity is available for the year, but can only range between $5 \mathrm{MW}$ and $50 \mathrm{MW}$ ), or 2 (new capacity in excess of a $50 \mathrm{MW}$ project may be available). For each site; the values at any time during the NEMS run will be consecutive "0's" from subscript 1 (1990) through the index for the year prior to initial new capacity availability, followed by either consecutive " 1 's" or " 2 's" from the initial year of new capacity availability through subscript 31 .

Values are first set during "first call" initialization in Subroutine GEINCAF, using the site's installed capacity (WGESICap ${ }_{P S, P Y}$ ) and planned capacity additions (WGESPCap ${ }_{P S, P Y}$ ) data reported to EIA and the site's potential capacity, WGESPt $t_{P S}$. Local, undimensioned variables are used for intermediate computations.

$$
\begin{array}{ll}
\text { WGESNCAv }_{P S, P Y}=0 & \text { for } P Y<I A Y \\
W G E S N C A v_{P S, P Y}=A V V A L & \text { for } \mathrm{PY}=I A Y \text { and }>I A Y
\end{array}
$$


where:

IAY = the initial year of new capacity availability at the site

$A v V A L=1$ if no more than $50 \mathrm{MW}$ can be built; -

2 if more than $50 \mathrm{MW}$ can be built

The computations for $A v V A L$ and $I A Y$ utilize the following "decision variable":

$$
D V=(T Y C+T P) / W G E S P t i P S
$$

where:

$T I C$ $=$, reported installed capacity

$T P$ $=\quad$ sum of all reported planned capacity additions

$D V$ represents total installed and planned capacity as a fraction of total potential capacity. $A V V A L$ is computed directly from $D V$. The other factor computed from $D V$-is $B D C o n$, a development constraint factor representing the minimum number of years between successive projects at a site.

CASE $1-D V$ between 0.15 and 0.85

$\begin{array}{llrl}\text { AvVal } & = & -1 & \text { More than a } 50 \mathrm{MW} \text { project may be available } \\ \text { BDCon } & = & 3\end{array}$. Minimum of 3 years between projects

CASE $2-D V \cdot$ either $<0.15$ or $>0.85$

$\begin{array}{llll}\text { AvVal } & = & 2 & \text { Project limited to no more than } 50 \mathrm{MW} \\ \text { BDCon } & = & 5\end{array}$

IAY is computed as follows:

CASE $1-D V=0(T I C=0$ and $T P=0)$

$$
I A Y=\max (9, Y+W G E P L E A D) .
$$

where:
$Y$
$=$. the index for the first year of the run
WGEPLEAD . = the lead time for new capacity 
CASE $2-D V>0$

$$
I A Y=\max (L P Y+B D C o n, Y+W G E P L E A D)
$$

where $L P Y$ is a year index computed as follows:

CASE $1-T P>0$

$L P Y=P 2$, the year of the last planned capacity addition

CASE $2-T P=0$, WGESICap ${ }_{P S, 1}>0$, all other non-zero WGESICap ${ }_{P S, P X}=$. WGESICAP $P S, 1$

$$
L P Y=1
$$

CASE $3-T P=0$ and not CASE 2

$$
L P Y=I L
$$

where:

$$
I L \quad=\quad \text { the last year of a change in reported installed capacity }
$$

Site Cost Computations. Site.cost computations use the base year (1990) costs, read from the site data file, that are produced off-line by a PC-based program, PC-GES. These, and updated site costs that are computed, are placed in variables with a dimension corresponding to "this year". (index =1) and "next year" (index =2). When read by Subroutine GERDSITE, the base year costs are placed into the "next year" position for initial updating. The site cost arrays include:

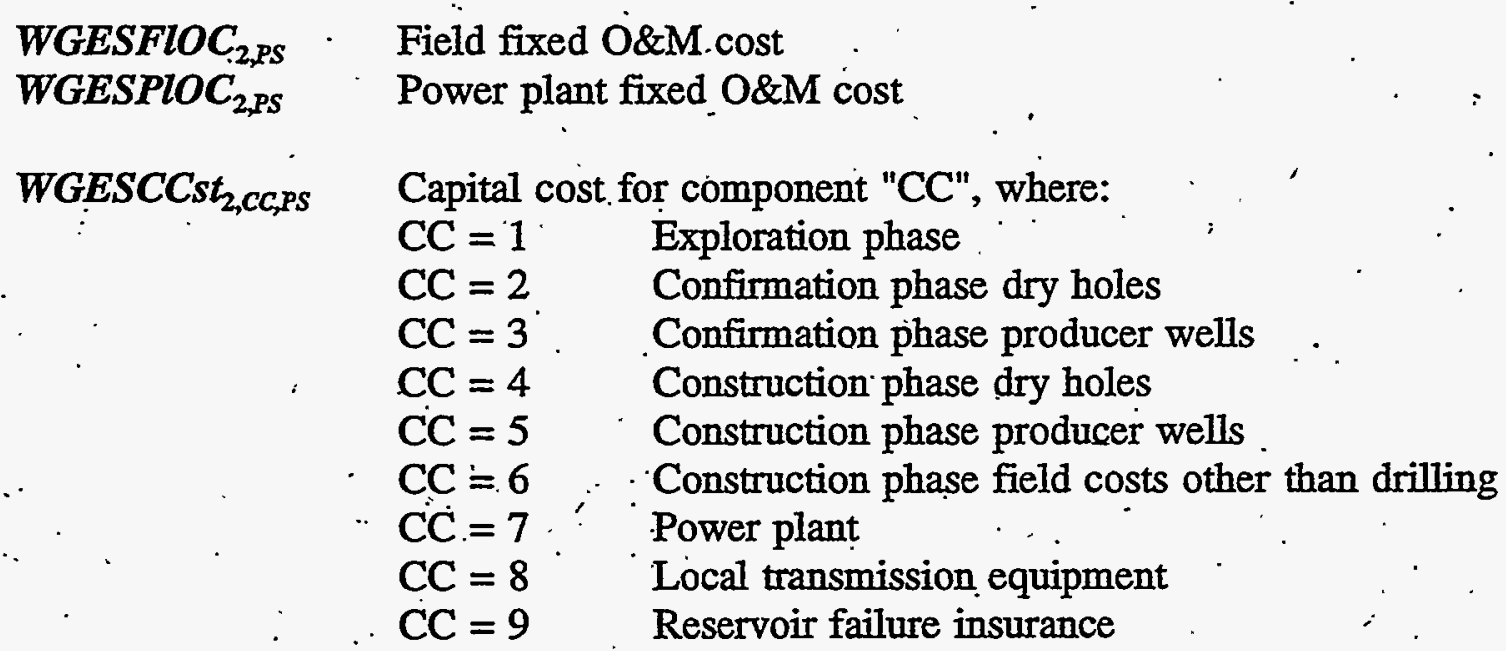

Values are read from the file for all but the capital costs for local transmission equipment and reservoir failure insurance, which are computed. The values are in units of millions of 1990 
dollars. Exploration phase capital costs represent a. one-time cost at the site preceding any development. All other costs are for a nominal $50 \mathrm{MW}$ plant.

Initial computations are made in Subroutine GERDSITE after the input data for a site is read. The principal computation is for costs related to supplemental wells that will be needed as enthalpy declines and replacements for failed wells. Site data such as unit well costs, lifetimes for producer and injector wells, beginning year and extent of enthalpy decline, and minimum flow requirements are used in this computation, which is detailed in the Appendix .7-B explanation of the GERDSITE algorithm. Using EMM economic parameter values, all capital costs and additional O\&M costs associated with supplemental and replacement wells are computed as an equivalent annual O\&M cost over the life of the project, TSPV, which is added as an increment to the value read for WGESFIOC 2,ps. $_{\text {. }}$.

The capital cost for local transmission equipment is computed as the product of a local transmission distance read from the site data file and a cost per mile key parameter. So that all transmission costs are accounted for in EMM, the site costs are set to 0 by setting the cost per mile parameter to zero.

All site costs are adjusted in Subroutine GERDSITE to convert their units from millions of 1990 dollars to thousands of 1987 dollars (multiplication by $1000 / M C \_P G D P_{1}$, the GDP deflator for 1990). All site costs are also adjusted to reflect derating due to enthalpy decline. The factor for this adjustment is the ratio of the net present "value" (but with an annual value of " 1 " per unit of output) of a constant annual output from a $50 \mathrm{MWe}$ plant over an assumed 30-year life to the same net present value computation for an output stream that declines over the last years of the plant, according to the specific initial year of decline and rate of decline values read for the site.

The site costs are updated to reflect technological/learning curve improvements over time in Subroutine GEUPSCO. For each of the site cost variables, the "next year" values with index 2 are first moved to the "this year" position with index 1 , and the new "next year" values computed as follows (where $N Y$ is the index for the "next year" and $\dot{P} T$ is the site's index value for type of plant -- binary or flash).

$$
\begin{aligned}
& \text { WGESFlÓC }_{2, P S}=\text { WGESFIOC }_{1, P S} * \text { WGEPGFOT }_{N Y} \\
& \text { WGESPLOC }_{2, P S}=\text { WGESPIOC }_{1, P S} * \text { WGEPGPOT }_{P T, N Y} \\
& \text { WGESCCst }_{2, c c, p s}=W G E S C C s t_{1, c c, p S} * W_{\text {GEPGDCT }} \quad \text { for CC }<6
\end{aligned}
$$

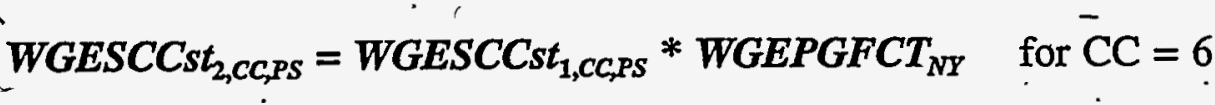

$$
\begin{aligned}
& \text { WGESCCst } t_{2, c C, P S}=\text { WGESCCst }_{1, C c P S} * \text { WGEPGPCT} T_{P T, N Y} \text { for } C \mathrm{C}=7 \\
& \text { WGESCCst }_{2, c C_{p} P S}=\text { WGESCCst }_{1, c c, p S} \quad . \quad \text { for CC }>7
\end{aligned}
$$

The factors used for updating power plant capital and O\&M costs are always those input as GES key parameters. However, when the key parameter $W G E T S W=1$, the key parameters input for 
'drilling and field cost update factors, are replaced by Oil \& Gas Supply Submodule factors. Using an OGSM algorithm, these are computed as a dimensioned $O G F$ variable, corresponding to an improvement index. Since this is $1 .+$ a percentage improvement, and the GES parameters represent the ratio of the new cost to the previous cost, the GES parameters are replaced as follows:

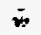

$$
\begin{array}{ll}
W G E P G D C T_{N Y}=2 .-O G F_{1} & \text { for updating drilling capital cost } \\
W G E P G F C T_{N Y}=2 \cdot-O G F_{2} & \text { for updating other field capital cost } \\
W G E P G F O T_{N Y}=2 .-O G F_{3} & \text { for updating field O\&M cost }
\end{array}
$$

The site costs in these variables are not used directly when costs for a site are needed for a computation. When site costs are needed for an algorithm, Subroutine GEADCST adjusts the drilling and other field capital costs for economic factors specific to geothermal projects, scales power plant costs, if necessary, for projects other than the nominal $50 \mathrm{MW}$, calculates reservoir failure insurance costs, converts the costs other than exploration capital costs to a $\$ / \mathrm{kW}$ unit cost for a particular capacity, and consolidates computed costs into the following variables with no dimensions:

$$
\begin{array}{ll}
\text { WGEAdXCC } & \text { Adjusted exploration capital costs' (units retained as thousand \$) } \\
\text { WGEAdFCC } & \text { Adjusted confirmation capital costs }(\$ / \mathrm{kW}) \\
\text { WGEEAdSCC } & \text { Adjusted construction phase capital costs }(\$ / \mathrm{kW}) \\
& \\
\text { WGEAdICC } & \text { Adjusted reservoir insurance capital costs } \\
\text { WGEAdOC } & \text { Adjusted total fixed O\&M costs }
\end{array}
$$

GEADCST has a single argument (CI in the subroutine), with a value of 1 if "this year" costs are to be used for the computations, or 2 if "next year" costs are to be used. PS, a variable in a Common block, provides the site index to be used. PQCAP, also a Common block variable, provides the amount of capacity for the project.

The tax rate used in making the economic factors adjustments is that obtained from EMM for the site's region: . .

$$
C T X R=T A X R T_{P N} \quad \text { where } P N=W G E S N R_{P S}
$$

The year index to be used for parameters dimensioned by year is set from the current NEMS . simulation year $\boldsymbol{Y}$ and the "processing year" $\boldsymbol{P Y}$ as follows:

$$
\begin{array}{ll}
D Y=Y+1 & \text { if } P Y>Y+W G E P L E A D \text { and } Y<26 \\
D Y=Y & \text { if } P Y \text { not }>Y+W G E P L E A D \text { or } \boldsymbol{Y}=26
\end{array}
$$


A combined factor is used to adjust for severance taxes, royalty payments, and depletion allowances:

$$
S R D F C t=\frac{1 .}{\left(1 .-W^{\prime}\right.}
$$

Computation results for initial adjustments are placed in local variables. For capital costs:

$$
\begin{aligned}
& \text { LACC }_{1}=\text { WGESXSS }_{P S} * \text { IntFct }_{1} * \text { SRDFct }
\end{aligned}
$$

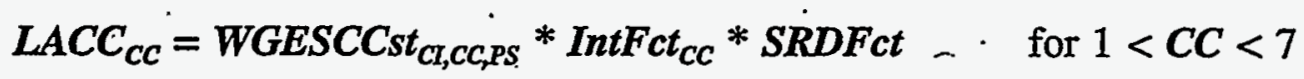

$$
\begin{aligned}
& L A C C_{\dot{c} C}=W_{G E S C C s t_{c,, c c, P S}} \text { IntFct }_{c c} \quad \text { for } C C \text { not }<7
\end{aligned}
$$

The IntF ct factor for all capital costs adjusts for the expensing of intangible capital expenditures, and is calculated from the tax rate, the percentage that is intangible for the particular capital.cost category, and the percentage of intangible cost that is expensed (currently 100 percent for all years), as follows:

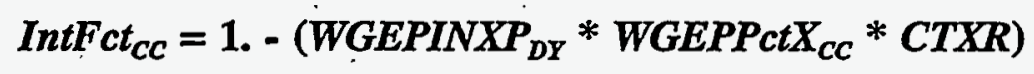

For O\&M costs,

$$
\begin{aligned}
& \text { LAFOC }=\text { WGESFIOC } C_{C I, P S} * \text { SRDFCt } \\
& \text { LAPOC }=\text { WGESPIOC } C_{C I, P S}
\end{aligned}
$$

Reservoir failure insurance cost is 5 percent of construction phase capital cost, which encompasses the components with index values 4 through 8 . Two scaling factors are needed for its computation:

$$
\begin{aligned}
& P S F=P Q C A P / 50 \quad \text {. if } P Q C A P<50 \\
& P S F=1 \text { if } P Q C A P \text { not }<50 \\
& P C S F=P S F^{07} \quad \quad \quad \text { if } P Q C A P<50 \text { and WGESPTy } p_{P S}=2 \\
& \text { (flash technology site) } \\
& P C S F=1 \quad \text { if } P Q C A P \text { not }<50 \text { or WGESPTyp } P S=1
\end{aligned}
$$

$P C S F$ is a scaling factor for both plant capital cost (index value 7) and plant O\&M cost and is needed because flash plant unit costs are dependent on plant size.

The cost of reservoir failure insurance, TRIC, is computed as 5 percent of the sum of the following terms: 


$$
\begin{array}{lc}
L A C C_{C C} * P S F & \text { for } C C=4,5,6,8 \\
L A C C_{7} * P S F & \text { if } P C S F=1 \\
L A C C_{7} * P C S F & \text { if } P C S F<1
\end{array}
$$

Exploration phase costs (retained in units of thousand \$) and confirmation phase costs ("unitized" to $\$ / \mathrm{kW}$ ) are independent of the scaling factors and may be computed directly:

$$
\begin{aligned}
& \text { WGEAdXCC }=\dot{L A C C} C_{1} \\
& \text { WGEAdFCC }=\left(L A C C_{2}+L A C C_{3}\right) / 50
\end{aligned}
$$

Cost components that are also independent of the scaling factors are initially consolidated into the construction phase capital costs and total O\&M costs:

$$
\begin{aligned}
& \text { WGEAdSCC }=L A C C_{4}+L A C C_{5}+L A C C_{6}+L A C C_{8} \\
& \text { WGEAdOC }=L A F O C
\end{aligned}
$$

Because any impact of the flash plant scaling factor is reflected in the calculation of TRIC, computation of unit reservoir insurance cost depends only on the PSF scaling factor:

$$
\begin{array}{ll}
\text { WGEAdICC }=\text { TRIC / } 50: & \text { if PSF }=1 \text { (QCAP is } 50 \text { or more) } \\
W G E A d I C C=T R I C / P Q C A P & \text { if } P S F<1
\end{array}
$$

Computations of unit capital costs for the construction phase and plant O\&M costs depend on both scaling factors:

$$
\begin{aligned}
& \text { WGEAdSCC }=\left(\text { WGEAdSCC }+L A C C_{7}\right) / 50 \quad \text { if } P S F=1 \\
& \text { WGEAdOC }=(\text { WGEAdOC + LAPOC }) / 50 \\
& W G E A d S C C=\left(W G E A d S C C+L A C C_{7} * P S F\right) / P Q C A P \\
& \text { if } P S F<1 \text { and } P C S F=1 \\
& W G E A d O C=(W G E A d O C+L A P O C * P S F) / P Q C A P \\
& \text { WGEAdSCC }=\left(\text { WGEAdSCC }+\mathrm{LACC}_{7} * \mathrm{PCSF}\right) / \mathrm{PQCAP} \\
& \text { if } P S F<1 \text { and } P C S F<1 \\
& \text { WGEAdOC }=(\text { WGEAdOC }+\angle A P O C * P C S F) / P Q C A P
\end{aligned}
$$


Two site cost computations relate only to new capacity planning. Exploration phase capital costs, incurred once at each site prior to initiation of capacity installation, must be allocated to individual projects at the site. This computation is primarily made in Subroutine GESCBLD to build new capacity supply curves. However, it is also used in Subroutine GECPDPR, which processes ECP build decisions, to "re-compute" costs for any sites for which a smaller project that is offered in the supply curve is to be built.

For sites with potential capacity greater than $300 \mathrm{MW}$, the costs are allocated uniformly over the first $250 \mathrm{MW}$ of installed capacity. All of the costs are allocated to the first installed capacity at sites with less potential capacity. Unless this is the case, exploration phase capital costs allocated to a project are computed as follows:

$$
\begin{aligned}
& W G E A d X C C=0 \text { if WGESPt } l_{P S} \text { not }>300 \text { and } T I C>0 \\
& \text { WGEAdXCC }=(\text { PQCAP } 1 \text { 1.250 }) * \text { WGEAdXCC } \\
& \text { if WGESPt| } \text { PS }_{\mathrm{PS}}>300 \text { and TIC not }>250 \text { and TIC+PQCAP not }>250 \text {. } \\
& \text { WGEAdXCC }=((250-T I C) / 250) * \text { WGEAdXCC } \\
& \text { if WGESPtl } l_{P S}>300 \text { and } T I C \text { not }>250 \text { and } T I C+P Q C A P>250
\end{aligned}
$$

where:

$$
\begin{aligned}
& T I C=\begin{array}{l}
\text { total previously installed capacity plus any planned capacity } \\
\text { additions }
\end{array} \\
& P Q C A P=\text { the amount of capacity being costed }
\end{aligned}
$$

After allocated exploration cost is computed for a project, the unit cost (units of $\$ / k \dot{W}$ ) is computed as:

\section{WGEAdXCC / PQCAP}

Generation of new capacity supply curves also entails computation of a levelized cost, in units. of cents per $\mathrm{kWh}$ for offered new capacity of $P Q C A P \mathrm{MW}$ at site $P S$. This computation is made in Subroutine GELEVEL when called by GESCBLD. Details. of the computation are in Appendix 7-B.

A levelized capital charge rate, $L \dot{A} F C R$, is computed from sinking fund depreciation, a retirement dispersion allowance, straight line depreciation income taxes, a double declining balance tax preference allowance, a flow through accounting tax preference allowance, and property tax and insurance. The capital charge rate is adjusted with an inflation factor, $G L V L$, in the computations.

Using financial functions, the future value of all capital costs as of plant start-up is aggregated as TFVCC. The future value computations use a 3-year confirmation phase and 2-year 
construction phase, rather the shortened total lead time of four years used to facilitate ECP's planning horizon.

Using the results of these preliminary calculations, levelized cost is calculated as:

$$
L C O S T=A D J *(T F V C C *(L A F C R / G L V L)+W G E A ̈ d O C) .
$$

where:

$$
A D J=\text { factor for conversion from annual } \$ / \mathrm{kW} \text { to cents per } \mathrm{k}: \mathrm{Wh} .
$$

Computations of New Capacity Amounts Offered at Sites. In generating new capacity supply curves, costs are computed for specific project sizes at specific sites. This section describes the computation of the project sizes, PQCAP. The computation uses the site's new capacity availability factor and uses the same decision variable, $D V$, used to determine the factor's value - total previously installed capacity and known capacity additions (planned or unplanned), TIC, as a fraction of the site's total potential capacity -- to determine PQCAP. This computation is performed in Subroutine GESCBLD.

For a given new capacity availability year; $\boldsymbol{P Y}$, the computation applies only to sites with a nonzero new capacity availability factor $\left(W G E S N C A v_{P S, P Y}\right)$. For these sites, a preliminary project size is computed as a percentage of total potential capacity as follows:

$$
\begin{aligned}
& N C Q=W G E S P t l_{P S} * 01 \quad \text { if } D V=0 \\
& N C Q=W G E S P t l_{P S} *(.01+(.14 / .15) * D V) \quad \text { if } D V>0 \text { and } D V \text { not }>.15 \\
& N C Q=W G E S P t l_{P S} * .15 \quad \text { if } D V>.15 \text { and } D V \text { not }>.8 \\
& \text { NCQ }=0.75 *\left(\text { WGESPtl } l_{P S}-\mathrm{TIC}\right) . \quad \text { if } D V>.8
\end{aligned}
$$

After rounding upward to the nearest $5 \mathrm{MW}$, the preliminary value is modified as indicated if one of the following constraints is violated (NCQ*.in the conditions refers to the preliminary value):

$$
\begin{array}{ll}
N C Q=20 & \text { if } W G E S N C A v_{P S, P Y}=1 \text { and } D V=0 \text { and } N C Q^{*}>20 \\
N C Q=50 & \text { if } W G E S N C A v_{P S, P Y}=1 \text { and } D V>0 \text { and } N C Q^{*}>50 \\
N C Q=10 & \text { if } W G E S N C A v_{P S, P Y}=1 \text { and } D V>0 \text { and } N C Q^{*}<10 \\
N C Q=200 & \begin{array}{ll}
\text { if } W G E S N C A v_{P S, P Y}=2 \text { and } N C Q^{*}>200 \\
N C Q=10 . & \text { if } W G E S N C A v_{P S, P Y}=2 \text { and } N C Q^{*}<10 \\
N C Q=50 & \text { if } W G E S N C A v_{P S, P Y}=2 \text { and } N C Q^{*}>50 \text { and } N C Q^{*}<70 \\
N C Q=100 & \text { if } W G E S N C A v_{P S, P Y}=2 \text { and } N C Q^{*}>100 \text { and } N C Q^{*}<120
\end{array}
\end{array}
$$




$$
N C Q=150 \text { if } \dot{W} G E S N C A v_{P S, P Y}=2 \text { and } N C Q^{*}>150 \text { and } \cdot N C Q^{*}<170
$$

If $N C Q$ is less than $50 \mathrm{MW}$ or is a multiple of $50 \mathrm{MW}(50,100,150$, or 200$)$, there is a single new capacity project offered at the site, with $P Q C A P=N C Q$. Otherwise, there will be two projects, each with its own calculated costs, which may differ due to the flash plant scaling factor or completion of the allocation of exploration phase capital cost. For these, the project sizes will be:

$$
P Q C A P=N C Q-M O D(N C Q, 50) \text { for the multiple of } 50 \mathrm{MW} \text { project }
$$

$$
P Q C A P=\operatorname{MOD}(N C Q, 50) \quad \cdot \quad \text { for the "residual" project }
$$

\section{Computations for NEMS Regions}

Most GES regional computations are for NERC regions. These typically map data for sites in a region into analogous data for the region as a whole. Such computations are made, successively for each NERC region in which any sites are located, with the initial computations made successively for each site in the region. The standard method for these computations uses the number of sites in a region to determine the site index for the last site in the region. The index for the first site in all regions after the first is simply the next index. value after that for the last site in the previous region. The standard method, then, may be summarized as follows:

Set last site index $(\boldsymbol{L S})$ to 0 .

Repeat for each NERC region with geothermal sites:

Set NERC region (PN) for computations

Set any initial values for region (generally accumulators set to 0 )

Set initial site index $F S$ to $L S+1$.

Increment last site index $(L S)$ by WGEINum $S_{P N}$

Repeat initial computations for each site from index $F S$ to $L S$

Perform final computations for region $P N$

There are also GES computations for Census regions, which are performed within the repetitive NERC region computational structure. Specifically,:

Prior to start of NERC region repetitions:- Set . initial values for all . Census regions

During initial site computation repetitions: Set Census region index $(C I)$ to $\boldsymbol{W} G E S C R_{P S}$. Update Census region $C I$ data with site $P S$ data

After end of NERC region repetitions:

Perform final computations for all Census regions

New Capacity Supply Curve Computations. For given year of availability, PY, new capacity supply curves are generated for each NERC region with geothermal sites. A supply curve's index, 
$S C$, is related to the corresponding NERC region's index, $P N$, by the value of WGEUNRgn SC. $_{\text {. }}$ A new capacity supply curve for each qualifying region is generated by four sets of computations in Subroutine GESCBLD.

In the initial computations, repeated over each site in the region, the computations for new capacity offered at the site, described above, identify either one or two potential projects for each site, PSS, with a non-zero new capacity availability factor for the year. Each project has a corresponding size, PQCAP, for which a levelized cost, $L C O S T$, is computed. If $L C O S T$ does not exceed a supply curve "truncation" value for the NERC region and availability year, WELSCST $_{P N, P Y}$, data characterizing the project are saved with the next "step" index, NS.

$$
\begin{aligned}
& N D \boldsymbol{X}_{N S}=\boldsymbol{N S} \\
& W \boldsymbol{D}_{N S}=\boldsymbol{P S} \\
& W L C_{N S}=\mathbf{L C O S T} \\
& W S Q_{N S}=\boldsymbol{P Q C A P}
\end{aligned}
$$

After the computations have been completed for each site in the region, the value of $N S$ is retained in WGEUNoSt $t_{S c}$. If 0 , no site had a non-zero new capacity availability factor, so no new capacity is available in the region. Values for all supply curve variables for the region also remain 0 and no further computations are performed for the region. Otherwise, Subroutine GESORT is performed. Its computations sort the levelized costs in WLC into ascending order, also adjusting the "tag" array, $N D X$, so that its step index values are placed in the same position as the project's levelized cost in the WLC array. The WID and WSQ arrays are not changed.

A third set of computations adjusts the sequencing in $W L C$ and $N D X$, if necessary, to ensure that a smaller project $(<50 \mathrm{MW}$ ) does not precede a larger project (multiple of $50 \mathrm{MW}$ ) at the same site, even if its levelized cost is less.

The final computations generate the supply curve data for the region: Variables used in the

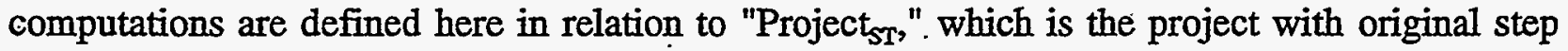
index $N X$, where:

$$
N X=N D X_{S T}
$$

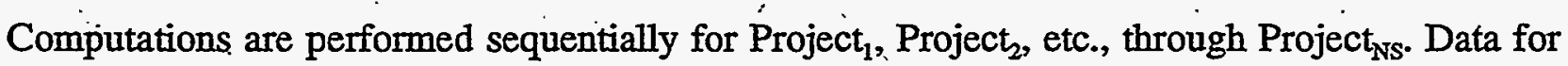
step 1 of the supply curve is for Project $t_{1}$ only; step 2.data is for Project ${ }_{1}$ and Project combined; $_{2}$ and so on, through the last step'with data for all NS projects combined. The site index and size for Project sT $_{\text {are: }}$

$$
\begin{array}{ll}
P S & =W D_{N X} \\
P Q C A P & =W S Q_{N X}
\end{array}
$$

These are used to compute costs for the project, using the previously described site data computations of Subroutine GEADCST. This, is also done in the first set of computations, to calculate the levelized cost of the project, but it is simpler to repeat the computations than retain 
the values of all cost components for all projects. Although values for undimensioned variables are produced by the subroutine and used in the computations, a subscript will be used for their mathematical description.

$O C_{S T}$ (for WGEAdOC) is the fixed O\&M cost for Project ST $_{\text {. }}$

$C C_{S T^{\circ}}$ (for WGEAäXCC + WGEAdFCC + WGEAdSCC + WGEAdICC) is the total overnight capital cost for Project ST $_{\text {. }}$

Other variables for the project are similarly subscripted here.

$P Q C A P_{S T}$ is the size of Project

$C F_{S T}=W G E S C F c t_{P S}$ is the capacity factor for Project $_{S T}$

$H R_{S T}=W G E S H t R t_{P S}$ is the heat rate for Project

$\operatorname{CO}_{2 T^{-}}=W G E S C O 2 R_{P S}$ is the $\mathrm{CO}_{2}$ emissions rate for Project

The site index for step $S T^{*}$ of supply curve $S C$ (for Nerc region WGEUNRgn $\dot{S C}$ ) is set as:

WGEVSI ${ }_{S C, S T^{*}}=P S$ (where $P \dot{S}=W D_{N X^{\prime}}$, where $\left.N X=N D X_{S T^{*}}\right)$

In all of the following computations, summations are over all projects from Project ${ }_{1}$ through Project $_{S^{*}}$, and the computations are made for all values of $S T^{*}$ from 1 through $N S$.

$$
\begin{aligned}
& W_{G E V N C C Q_{S C, S T^{*}}}=\sum P Q C A P_{S T} \\
& \text { WGEVCFCit } \text { SC;ST }^{*}=\sum\left(P Q C A P_{S T} * C F_{S T}\right) / \sum P Q C A P_{S T} \text { : } \\
& \text { WGEVOVCC }_{S C, S T^{*}}=\sum\left(P_{Q C A P} * O C_{S T}\right) / \sum P Q C A P_{S T} \\
& \text { WGEVFOC }_{S C S T^{*}}=\sum\left(P Q C A P_{S T} * C F_{S T}\right) / \sum P Q C A P_{S T} \\
& \text { WGEVHtRt } t_{S C, S T^{*}}=\sum\left(P Q C A P_{S T} * C F_{S T} * H R_{S T}\right) \cdot / \sum\left(P Q C A P_{S T} * C F_{S T}\right) \\
& \text { WGEVCO2R } R_{S C, S T^{*}}=\sum\left(P Q C A P_{S T} * C F_{S T} * C O 2 R_{S T}\right) / \sum\left(P Q C A P_{S T} * C F_{S T}\right)
\end{aligned}
$$

New. Capacity Planning Output Computations. New capacity planning output for ECP, produced with Subroutine GECPOUT, is taken directly from the supply curve data, but is limited to data for a single point on the "curve." Accordingly, the output variables are dimensioned only" by NERC region and year. A set of "local" variables for the output values are defined so that the year index corresponds to the initial year of availability if the new capacity is built. These variables are used in all GES computations and are consistent with the year index interpretation for the corresponding ECP new capacity build decision. However, a different year index interpretation is used for the global variables from which EMM obtains the values. For the 
interface variables that have been established for all renewable technologies, the year index corresponds to the year prior to that for which ECP. uses the data for its new capacity planning. From the 4-year lead time for new geothermal capacity, the GES availability year index, $P Y$, translates into a year index of $\boldsymbol{P Y}-5$ for the global interface variables.

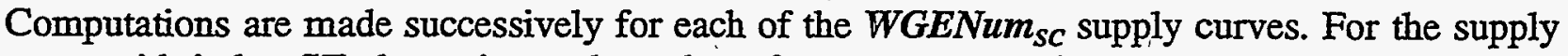
curve with index $S T$, the region and number of steps are set as follows:

$$
\begin{aligned}
& P N=W G E U N R g n_{s c} \\
& R S=W G E U N R g n_{S C}
\end{aligned}
$$

The upper bound on the amount of new capacity that can be built is the total capacity of all projects, computed for the last step.

$$
W C A G P E L_{P N, P Y .5}=W G E P M A X C_{P N, P Y}=W G E V N C C Q_{S C, R S}
$$

Other output values may also correspond to those for the last step, but there are circumstances under which a different step is used for these values. The reason is that the ECP linear programming model treats the unit costs provided by the GES as constant for all build amounts. Unit costs in GES are not fixed and, by construction, increase from one step to the next. Costs for the last step are the maximum unit costs. If only these maximum costs are provided to the ECP; build decisions will never reflect the lower costs if less than the maximum available new capacity is built. Therefore, after the first ECP build decision, when there are more that four steps in a supply curve and the ECP build decision for the previous year was less than the maximum amount available, the value of $R S$, the step that is used for the output, is changed to:

$$
\boldsymbol{R S}=W G E U N R g n_{S C} / 2 \text { (rounded down if there is an odd number of steps) }
$$

The additional new capacity planning output is set as follows:

$$
\begin{aligned}
& W C F G P E L_{P N, P Y-5}=W G E P C F_{P N, P Y}=W G E V C F C t_{S C, R S} \\
& W C C G P E L_{P N, P Y-5}=W G E P C C_{P N, P Y}=W G E V O V C C_{S C, P S} \\
& W O C G P E L_{P N, P Y-5}=W G E P F X O C_{P N, P Y}=W G E V F O C_{S C, R S} \\
& W H R G P E L_{P N, P Y-5}=W G E P H t R t_{P N, P Y}=W G E V H t R t_{S C, R S} \\
& W E M G P E L_{3, P N, P Y-5}=W G E P C O 2 R_{P N, P Y}=W G E V C O 2 R_{S C, R S}
\end{aligned}
$$

Other New Capacity Output Related Computations. New capacity supply curves are built and output for ECP produced by setting the year of availability, $P N$, then running Subroutines GESCBLD and GECPOUT. As a special case, this is done in Subroutine RFMGES for $P Y=$. FIRSYR + 4 (unless FIRSYR is 1. - for 1990 - which would result in a 0 year index 
" $(1+4-5)$ for the global output variables). This special case produces the data needed to process any ECP build decision made that year, before RFM is first run.

Apart from this special case, Subroutine GECPOPR controls the capacity planning output processing. Specifically, for each year, supply curves are built and output for ECP produced for each year of the ECP planning horizon for the decisions to be made the following year. When GES runs for simulation year $Y$, the planning horizon for the following year covers years $Y+1$ through $Y+6$. The initial year of availability for geothermal new capacity is $(Y+1+4)$ or $Y+5$. Supply curves are generated and ECP output produced, first for $\boldsymbol{P Y}=\boldsymbol{Y}+5$, and then for $\boldsymbol{P Y}=$ $Y+6$.

For the $P Y=Y+6$ computations, it is assumed that all new capacity offered for $Y+5$ in all NERC regions is built. Before this is done, it is first necessary to save all of the supply curve data for the initial year, as well as all site new capacity availability factors and unplanned capacity amounts. Then, new site availability factors are computed for use in the $Y+6$ computations, using the maximum available being built assumption. Lastly, after ECP output is produced for the last year of the planning horizon, the saved site new capacity availability factors and unplanned capacity values, and $\boldsymbol{P Y}=\boldsymbol{Y}+5$ supply curve data are restored.

Computations for Processing ECP New Capacity Build Decisions. The computations for processing ECP new capacity build decisions are in Subroutine GECPDPR. No computations are made for regions where no new capacity is to be built. Where any new capacity is to be built, $\boldsymbol{C P}$ and $\boldsymbol{C P Q}$ are computed initially, where:

$C P$ is the maximum step index $S T$ such that $W G E V N C C Q_{S C, S T}$ does not exceed the amount to be built.

$$
C P Q=W G E V N C C Q_{S C, C P}
$$

If $C P Q$ is less than the amount to be built, $\boldsymbol{C P}$ is incremented by 1. Only part of the additional capacity in this step will be built.

The principal computations are done sequentially by step, from 1 through $C P$. For step $S T$, the site index and amount of new capacity offered from the site are:

$$
\begin{aligned}
& \text { PS }=W G E V S I_{S C, S T} \\
& \begin{array}{lll}
P Q C A P & =W G E V N C C Q_{S C, S T} &
\end{array} \\
& P Q C A P=W G E V N C C Q_{S C, S T}-W G E V N C C Q_{S C, S T-1} \quad \text { for } S T>1
\end{aligned}
$$

With the possible exception of the last step, PQCAP is also the amount to be built at the site. If less than the capacity offered in step $\boldsymbol{C P}$ is to be built, the amount to be built at the site is the difference between the amount to be built in the region and $C P Q$, so $P Q C A P$ is recomputed as this value. 
Site unplanned capacity additions are computed with an incremental formula, since two different - projects may be built at a site (one a multiple of $50 \mathrm{MW}$ and the other < $50 \mathrm{MW}$ ):

$$
\text { WGESUCAP }_{P S, P Y}=\text { WGESUCap } P S, P Y=P Q C A P .
$$

Site new capacity availability factors are updated to reflect the information for the next available new capacity at the site. The algorithm is the same as is used to set the initial values during initialization.

The output used by ECP in making the decision, other than the build limit, is modified if necessary so that it corresponds to the amount being built. If less then the maximum available new capacity is being built, the site costs for the additional capacity to be built from step $\boldsymbol{C P}$ may be different from the costs for the additional amount offered, requiring adjustment before the revised ECP output can be computed.

To make the adjustment, it is necessary to break the capital cost down into its different components. Initially, the original added total capital cost is saved and costs are computed for the offered quantity. Where $P S=W G E V S I_{S c, C P}$ :

$$
\begin{array}{ll}
P Q C A P & =W G E V N C C Q_{S C, C P} \\
U C C A D J & =W G E V O V C C_{S C, S P}
\end{array}
$$

UCCADJ =

$\left(W G E V N C C Q_{s c, C P} * W G E V O V C C_{S C, C P}\right)-\left(W G E V N C C Q_{s c, C P-1} * W G E V O V C C_{S C, C P-1}\right)$

$$
P Q C A P
$$

The original construction phase capital costs (WGEAdSCC) and reservoir failure.insurance capital cost (WGEAdICC) are subtracted from UCCADJ, leaving the sum of confirmation phase and any exploration phase capital costs. These unit costs $(\$ / \mathrm{kW})$ do not vary with size. PQCAP is changed to the amount to be built and the unit costs for this quantity are computed with Subroutine GEADCST. Adding the new values of WGEAdSCC and WGEAdIOC to UCCADJ gives total unit capital costs for the new quantity

If the computations are for the first step $(\boldsymbol{C P}=1)$, there are no other projects to be built. Heat and emissions rates are fixed for the project site, so only the two cost values need to be computed.

$$
\begin{aligned}
& \text { WGEVOVCC } C_{S C, C P}=\text { UCCADJ + WGEAdSCC + WGEAdIOC } \\
& \text { WGEVFOC } C_{S C, C P}=\text { WGEAdOC }
\end{aligned}
$$


When $C P>1$, other projects are to be built. All output data must be adjusted to reflect the "last step's" smaller percentage of the total amount to be built in the region than in the original supply curve computations. Letting WELRQCA represent the ECP build decision for the region:

$$
\begin{aligned}
& \text { (WGEVNCCQ } \left.Q_{S G, C P-1} * W G E V O V C C_{S C, C P-1}\right) \\
& \text { WGEVOVCC }_{S C, C P}=\frac{+P Q C A P *(U C C A D J+W G E A d S C C+W G E A d I O C)}{\text { WELRQCA }}
\end{aligned}
$$

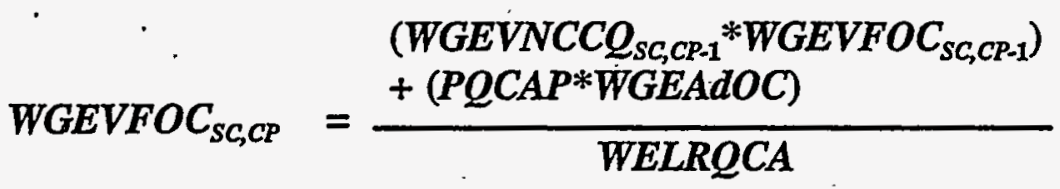

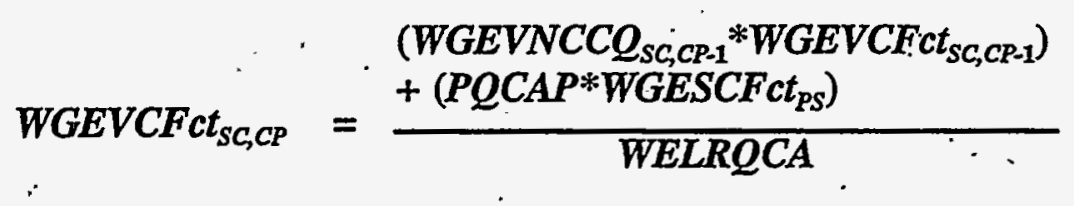

$$
\begin{aligned}
& \text { (WGEVNCCQ } \left.Q_{S C, C P-1} * W G E V C F C t_{S C, C P-1} * W G E V H t R t_{S C, C P-1}\right)
\end{aligned}
$$

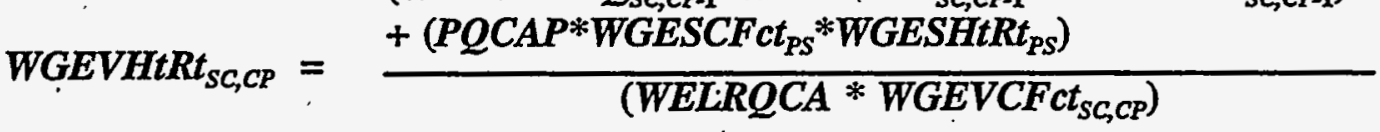

$$
W G E V C \dot{2} 2 R_{S c, C P}=\frac{\begin{array}{l}
\left(W G E V N C C Q_{S C, C-1} * W G E V C F C t_{s C, C P-1} * W G E V C O 2 R_{S C, C P-1}\right) \\
+\left(P Q C A P * W G E S C F C t_{P S} * W G E S C O 2 R_{P S}\right)
\end{array}}{\left(W E L R Q C A * W G E V C F t_{S C, C P}\right)}
$$

All local and global new capacity output variables except those for the build constraint are set to the corresponding value computed for step $C P$. The fixed O\&M cost for the new capacity to be built in NERC region $P N$ with initial availability in year $P Y$ is saved for later use in computing output for all installed capacity for year $\boldsymbol{P Y}$.

$$
W G E N U O C_{P N, P Y}=W G E P F X O C_{P N, P Y}
$$

Dispatch Related Output Computations for All Installed Capacity. Regional (NERC and Census) output for all installed capacity. in 1990 is computed as part of the initialization process with Subroutine GEINTRG. Output for later years may also be computed during initialization, or may be computed as part of the standard processing for every year with. subroutine GEEDOPR. The determining factor is the first simulation year index, FIRSYR. If FIRSYR is 1 or 2, output for all years after 1990 is computed with GEEDOPR. Otherwise, output for all years after 1990, but prior to FIRSYR, is computed with GEINTRG during initialization; output: 
for all years starting with $F I R S Y R$. is computed with GEEDOPR. Output for all installed capacity for FIRSYR (if $>1$ ) is computed as part of the special processing for that year in Subroutine RFMGES. The standard procedure for all years (with index $<26$ ) produces output for the following year (FIRSYR+1 output when run for FIRSYR, etc.)

There are only minor differences between the computations performed in GEINTRG and in GEEDOPR. In both cases, one of the computations for years after 1990 updates the installed capacity at each site. The primary difference is that the GEEDOPR computations update for both planned and unplanned capacity additions, while the GEINTRG update is limited to planned capacity additions.

$$
\begin{aligned}
& \text { WGESICap }_{P S, P Y}=\text { WGESICap }_{P S, P Y-1}+\text { WGESPCap }_{P S, P Y} \\
& \text { (GEINTRG) } \\
& \text { WGESICap }_{P S, P Y}=\text { WGESICap }_{P S, P Y-1}+\text { WGESPCap }_{P S, P Y}+\text { WGESUCap }_{P S, P Y} \cdot(G E E D O P R)
\end{aligned}
$$

As usual, the computations are made sequentially for each NERC region, with the initial computations performed sequentially for the sites in the region. Totals are aggregated for both NERC regions and Census regions.

Except for fixed O\&M cost, by NERC region, the output computations aggregate fixed site parameters that do not change over time. These computations are generally the same for all years and in both subroutines. The only variability is due to the requirement to reflect data for The Geysers in the output for its NERC region and Census region. Initial computations when processing each site in successive NERC regions are identical.

For the one NERC region computation, summation is over all sites in the region being processed.

$$
\text { NRCRFCT }=\Sigma\left(\text { WGESICap } \text { PSSPY }^{*} \text { WGESCFCt }_{P S}\right) \text {. }
$$

For the Census region computations, summation for region $C I$ is over all sites in all NERC regions such that WGESCR$R_{P S}=C I$.

$$
\begin{aligned}
& \operatorname{CRCRFCT}_{C I}=\Sigma\left(\text { WGESICap }_{P S, P Y} * \text { WGESCFCt }_{P S}\right)
\end{aligned}
$$

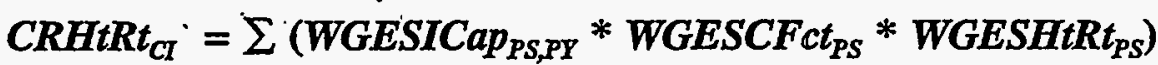

$$
\begin{aligned}
& \operatorname{CRECO2}_{C I}=\sum\left(\text { WGESICap }_{P S, P T} * \text { WGESCFCt }_{P S} * \text { WGESCO2R }_{P S}\right)
\end{aligned}
$$

NERC region output is produced after all sites in the region have been processed: Total installed capacity in the region is:

NRICAP $=\Sigma$ WGESICap $P S, P Y$

CASE $1-N R I C A P=0$ and $\dot{P} N$ not $=W G E G N R$

No computations, output value remains 0 . 
CASE $2-N R I C A P=0$ and $P N=$ WGEGNR

$W C F G I E \dot{L}_{P N, P Y}=W G E G C F c t_{P Y} \cdot$ (capacity factor for $\cdot$ The Geysers fór year $P Y$ )

CASE $3-N R I C A P>0$ and $P N$ not $=$ WGEGNR

WCFGIEL $L_{P N, P Y}=$ NRCFCT ! NRICAP

CASE $4-N R I C A P '>0$ and $P N=W G E G N R$

$$
W_{C F G I E L_{P N, P Y}}=\frac{\left(N R C F C T+W G E G I C A P_{P Y} * W G E G C F c t_{P Y}\right)}{\left(N R I C A P+W G E G I C A P_{P Y}\right)}
$$

Census region output is produced after all sites in all regions have been processed. Total installed capacity in Census region $\boldsymbol{C I}$ is:

CRICAP $_{C I}=\sum$ WGESICAP $_{P S, P Y}$

CASE $1-C R I C A P_{C I}=0$ and $C I$ not $=$ WGEGCR

No computations, output value remains 0 .

CASE $2-C R I C A P_{C I}=0$ and $C I=W G E G C R$.

WHRGIEL $L_{C, P Y}=$ WGEGHtRt

WEMGIEL $L_{3, C I, P Y}=W G E G C O 2 R$

CASE $3-C R I C A P_{C I}>0$ and $C I$ not $=W G E G C R$

$$
\begin{aligned}
& \text { WHRGIEL }_{C, P Y}=\text { CRHTRT }_{C I} / \text { CRCFCT }_{C I} \\
& \text {. WEMGIEL } L_{3, C I, P Y}=\text { CRECO2 }_{C I} / \text { CRCFCT }_{C I}
\end{aligned}
$$

CASE $4-C R I C A P_{C I}>0$ and $C I=W G E G C R$

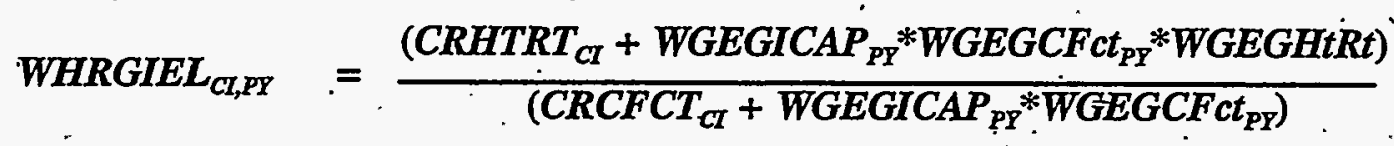

$$
\begin{aligned}
& W E M G I E L_{3, \mathrm{CL}, \mathrm{PY}}=\frac{\left(C R E C O 2_{C Y}+W G E G I C A P_{P Y} * W G E G C F C t_{P Y} * W G E G C O 2 R\right)}{\left(C R C F C T_{C Y}+W G E G I C A P_{P Y} * W G E G C F C t_{P Y}\right)}
\end{aligned}
$$

Fixed O\&M cost computations are not the same under all circumstances. For 1990, the computations are analogous to that for output related to fixed site data. The computations aggregate the costs for installed capacities. For the initial computations, PQCAP is set to 
WGESICAP $\dot{p}_{P S, 1}$ and the computations of Subroutine GEADCST produce the costs for the capacity. NRFXOC is computed as the sum of the product of PQCAP and WGEAdOC. After all sites in the region have been processed:

CASE $1-N R I C A P=0$ and $P N$ not $=W G E G N R$

No computations, output value.remains 0 .

CASE $2-N R I C A P=0$ and $P N=W G E G N R$

WOCGIEL $L_{P N, P Y}=W G E G O C$

CASE $3-$ NRICAP $>0$ and PN not = WGEGNR

WOCGIEL $L_{P N, P Y}=$ NRFXOC / NRICAP.

CASE $4-N R I C A P>0$ and $P N=W G E G N R$

$$
W C F G I E L_{P N, P Y}=\frac{\left(N R F X O C+W G E G I C A P_{P Y} * W G E G O C\right)}{\left(N R I C A P+\cdot W G E G I C A P_{P Y}\right)}
$$

The general approach for years after 1990 is to compute fixed O\&M cost as a weighted average of the cost of previously installed capacity and any new capacity additions. In Subroutine GEINTRG, only planned capacity additions need be considered. The initial computations are the same as for 1990 , except that PQCAP is set to WGEGPCap WS $_{\text {,PY }}$, so that the computation for NRFXOC produces total OQM costs of all planned capacity additions for the region.

It there is no capacity installed in the region (either previously installed or as new capacity additions), the cost, as for 1990, is 0 (no change from initialization value). except for The Geyser's region; or, for that region, WGEGOC. For "CASE 3" and "CASE.4":

CASE $3-\dot{W} G E N I C A P^{\prime}>0$ and $P N$ not $=$ WGEGNR

$$
W O C G I E L_{P N, P Y}=\frac{\left(N R F X O C+W O C G I E L_{P N, P Y-1} * W G E N I C a P_{P N, P Y-1}\right)}{W^{\prime} W E N I C A P_{P N, P Y}}
$$

CASE $4-W G E N I C A P>0$ and $P N=W G E G N R$

$$
\text { WOCGIEL } L_{P N, P Y}=
$$$$
\left(N R F X O C+W O C G I E L_{P N, P Y-1} *\left(W G E N I C a \dot{p}_{P N, P Y-1}+W G E G I C A P_{P Y=1}\right)+A\right.
$$

$$
\text { (WGENICAP } \left.P_{P N, P Y}+W G E G I C A P_{P Y}\right)
$$


where:

$$
A=W G E G O C *\left(W G E G I C A P_{P Y}-W G E G I C A P_{P Y-1}\right)
$$

This term is always zero in the current version of GES, but it is included so the computation willbe made correctly if installed capacity at The Geysers changes over time in a future version.

The same initial computations for planned capacity additions are made in Subroutine GEEDOPR, but there additional computations are required for unplanned new capacity additions. Only the total unplanned capacity addition, NRUCAP, need be computed. The fixed O\&M cost for these capacity additions are saved when the ECP capacity planning decision was made. For total fixed O\&M costs for unplanned capacity additions:

$$
N R U F O C=N R U C A P * W G E N U O C_{P N, P Y}
$$

The computations are so similar there is no need to state the final computations of fixed O\&M cost output. It is only necessary to replace total costs for planned additions (NRFXOC) with the total cost of planned and unplanned additions, which is (NRPFOC + NRUFOC). 



\section{Appendix 7-A: Inventory of Variables, Data and Parameters}

Due to its size, Appendix 7-A has not been included here. The reader is referred to the report,

"Model Documentation: Geothermal Electric Submodule of the Renewable Fuels Module of the National Energy Modeling System," December 1994, prepared by DynCorp-Meridian Inc. for the Energy Information. Administration. The Inventory of Variables, Data, and Parameters is in Appendix A, pp. 27-176. A copy of the report is.maintained in the files of:

\section{Roger Diedrich}

U.S. Department of Energy/EIA

1000 Independence Avenue, S.W.

Room 1E-256 (EI-822)

Washington, DC 20585

(202) 586-0829 


\section{Appendix 7-B: Mathematical Description}

Due to its size, Appendix 7-B has not been included here. For Appendix 7-B, the reader is referred to the report, "Model Documentation: Geothermal-Electric Submodule of the Renewable Fuels Module of the National Energy Modeling System," December 1994, prepared by DynCorpMeridian Inc. for the Energy Information Administration. The Mathematical Description is given in Appendix B; pp. 177-225. A copy of the report is maintained in the files of:

Roger Diedrich

U.S. Department of Energy/EIA

1000 Independence Avenue, S.W.

Room 1E-256 (EI-822)

Washington, DC 20585

(202) $586-0829$ 



\section{Appendix 7-C: Bibliography}

\section{A. NEMS Development Documents}

Energy Information Administration, "System Design for the National Energy Modeling System" (January 16,1991 Draft).

Energy Information ,Administration, "Integrating Module Component Design Report" (September 4, 1992).

Energy Information Administration, "Component Design Report: Macroeconomic Activity Module, National Submodule" (May, 1992).

Energy Information Administration, "Component Design Report: Electricity Capacity Planning" (August 13, 1992 Draft).

Energy .Information Administration, "Component Design Report: Electricity Fuel Dispatch" (June, 1992 Draft).

Energy Information Administration, "Component Design Report: Electricity Financing and Pricing Submodule" (June 22, 1992 Draft).

Energy Information Administration, "Component Design Report: Nonutility Generation Supply" (May 11, 1992 Draft).

Energy Information Administration, "Component Design Report: Modeling Technology Penetration" (November 6, 1992 Draft).

Energy Information Administration, "Component Design Report: Basic Framework \& Onshore Lower 48.Conventional Oil and Gas Supply" (April 23, 1992 Draft).

Energy Information Administration, "Component Design Report: Geothermal Electricity Submodule" (December 1992, Draft)

\section{B. Other References}

Bloomquist, R., Black, G., Parker, D., Sifford, A.; Simpson, S., and Street, L., "Evaluation and Ranking of Geothermal Resources for Electrical Generation or Electrical Offset in Idaho, Montana, Oregon and Washington,". Bonneville Power Administration, Vol. I and II . (1985).

Energy. Information Administration, "Geothermal Energy in the Western United States and Hawaii: Resources and Projected Electricity Generation Supplies" (August, 19.91) 
Entingh, D. and McLarty, L., "Geothermal Cost of Powwer Model IM-GEO Version 3.05: User's Manual" (February 22, 1989).

Environmental Protection Agency, ICF Incorporated Contractor Report, "Renewable Electricity Generation: An Assessment of Air Pollution Prevention Potential" (March, 1992).

Jelen, F. and Black, J., "Cost and Optimization Engineering," 2nd Edition (McGraw-Hill, Tokyo, 1983).

Muffler, L., Ed., "Assessment of Geothermal Resources of the United States - 1978," United : $\quad$ States Geological Survey Circular 790 (1979):

Petty, S., Entigh, D., and Livesay, B., "Impact of R\&D on Cost of Geothermal Power," prepared for Sandia National Laboratory (1988).

Petty, S., Livesay, B., Long, W. and Geyer, J., Contractor Report, "Supply of Geothermal Power from Hydrothermal Sources: A Study of the Cost of Power in 20 to 40 Years" (June 21, 1991).

White, D. and Williams, D., Eds., "Assessment of Geothermal Resources of the United States 1975," United States Geological Survey Circular 726 (1975).

McLarty, L., "Geothermal Electric Supply Data Pre-Processor: Model Documentation," (November 1994 Draft). 


\section{Appendix 7-D: Model Abstract}

\section{Model Name:}

Geothermal Electric Submodule

\section{Model Acronym:}

GES

\section{Description:}

The GES models economic supply curves, aggregated by NERC region, for electric generation capacity utilizing known U.S. geothermal hydrothermal resources. The costing algorithms model the impacts of specific resource site parameters at known geothermal sites on the capital cost, operation and maintenance cost, and energy prices at those sites. For each year, GES processes new capacity build decisions made by the Electric Capacity Dispatch (ECP) submodule, allocating the regional build amounts to individual sites in the region through the new capacity supply curve data for the region; updates installed capacity and related data for both sites and regional aggregations, and produces output for use by the Electric Fuel Dispatch submodule; and produces new capacity supply curves and related output for use by the ECP submodule.

\section{Purpose of the Model:}

The purpose of GES is to supply the Electric Market Model (EMM) of NEMS with geothermal supply curves and annual capacity information for dispatch.

\section{Most Recent Model Update:}

July 1994

\section{Part of Another Model?:}

The GES submodule is a component of the Renewable Fuels Module (RFM) of the National Energy Modeling System (NEMS).

\section{Official Model Representative:}

Roger Diedrich

Coal, Uranium, and Renewable Fuels Analysis Branch

Energy Information Administration

Phone: (202) 586-0829

\section{Documentation:}

Model Documentation: Renewable Fuels Module of the National Energy Modeling System, 1995.

\section{Archive Media and Installation Manual(s):}

Archived as part of the NEMS production runs. 


\section{Energy System Described:}

Complete systems for extracting geothermal heat from the earth and converting it to electricity, including wells, fluid gathering systems, both binary and flash rankine cycle energy conversion systems, and fluid reinjection systems.

\section{Coverage:}

- Geographic: NERC regions 11, 12, 13, and 15 .

- Time Unit/Frequency: Annual, 1990 through 2015

- Products: Electricity

- Economic Sectors: Electric utility

\section{Modeling Features:}

- Model Structure: The model operates at the level of individual geothermal sites. Regional inputs from the Electricity. Market Module are disaggregated to the individual sites and are operated upon at that level. Results are then rolled up to the regional level at which NEMS is structured for output.

- Model Technique: Risk avoidance model of developer behavior is used to determine timing and extent of further development at each site. New capacity supply curves are generated in terms of minimizing levelized cost for any new capacity amounts. Regional aggregate data is computed as weighted average of analogous individual site data, using either capacity or generation as weights, as appropriate.

- Special Feature:

- Accommodates runs for either type of looping. Incorporates dynamic interface in which the output needed by the EMM for processing the next year is produced, rather than the output for the current year.

- Employs a PC-based data pre-processor to calculate site-specific costs and performance of geothermal systems based on resource data for 51 known U.S. geothermal resource sites.

\section{Non-DOEE Input Sources:}

None. 


\section{DOE Input Sources:}

Petty, Susan, et al, Supply of Geothermal Power from Hydrothermal Sources: A Study of the Cost of Power in 20 and 40 Years, Susan Petty Consulting, Solana Beach, CA, June 1991 for the Energy Information Administration and Geothermal Division of DOE.

- Geothermal Resource Site Data

Entingh, D., McLarty L., Nichols, K. Livesay, W., and Petty. S., "Geothermal Cost of Power Model IM-GEO Version 3.05" developed for Sandia National Laboratory and the Geothermal Division of the DOE.

- Geothermal systems operational and cost parameters and algorithms.

\section{Computing Environment:}

$\therefore$ Hardware Used: IBM 3090

- Operating System: MVS

- Language/Software used: VS Fortran, Version 2.05

- Memory Requirement: $35 \mathrm{~Kb}$

: Storage Requirement: $23 \mathrm{~Kb}$

- Estimated Run Time: :08 seconds

- Special Features: None.

\section{Independent Expert Reviews Conducted:}

None.

\section{Status of Evaluation Efforts by Sponsor:}

None. 


\section{Appendix 7-E: Data Quality and Estimation Processes}

This appendix provides an overview of the Geothermal Electric Supply Data Pre-Processor model, or GESDPP. A PC-based model, GESDPP serves as the main source of input data used in the GES. It is adapted from an earlier PC-based model, IM-GEO ${ }^{23}$, developed by a team of geothermal industry experts for Sandia National Laboratory in the mid 1980s. "IM-GEO".stands for Impacts of Research and Development on the Cost and Performance of Geothermal Electric Systems:

The GESDPP, calculates typical capital and operation and maintenance costs (as well as heat rates, flow rates, and other technical parameters) for a single $50 \mathrm{MW}$ (net) plant at each of 51 known geothermal resource sites in the western U.S., including Hawaii. Based on historical cost data for independent geothermal power projects developed during the 1980s, GESDPP models existing, commercially available technologies associated with power generation from hydrothermal resources. To date, hydrothermal resources are the only geothermal resources for which.commercial development has been feasible.

The main input to GESDPP ì à geothermal resource data set which includes data on 51 known geothermal resource sites in the U.S. The site data include measured parameters as well as parameters which were estimated based on other measured data for the site in question or on more complete knowledge of a geologically and hydrologically similar site. These data were compiled during a study completed in 1991 for EIA and the Geothermal Division of DOE ${ }^{24}$, using U.S. Geological Survey data and other sources.

The cost and performance data and algorithms within GESDPP are considered accurate since costs as modeled by IM-GEO have been found to be reasonably similar to costs for actual. geothermal projects. The resource site data are considered less reliable. For the 1.7 resource sites with existing.geothermal capacity, and especially for those that have been operating for several years, the data are probably quite reliable. However, for most of the other sites, geological, geophysical and hydrological investigations have been limited, and much of the data have been estimated based on knowledge of geothermal resource sites with similar general geological conditions.

Immediately following are general flow diagrams for GESDPP, presented in Figures 7E-1, $7 \mathrm{E}-2$, and 7E-3, and a listing of mathematical descriptions of the key costing and performance algorithms. A list of the input resource data and output data from GESDPP are presented in Part 3 of Appendix 7-A. A description of the output file, WGESTTE; which serves as the main input file for GES can also be found in Appendix 7-A.

\footnotetext{
${ }^{23}$ Entingh, D.J. and Mclarty, L. Geothermal Cost of Power Model-IM-GEO Version 3.05: User's Manual, for Sandia National Laboratory, November 1991.

${ }^{24}$ Petty, Susan, et al, Supply of Geothermal Power from Hydrothermal Sources: A Study of the Cost of Power in 20 and 40 Years, Susan Petty Consulting, Solana Beach, CA, June 1991.
} 
Figure 7E-1. Main Flow Chart of GESDPP

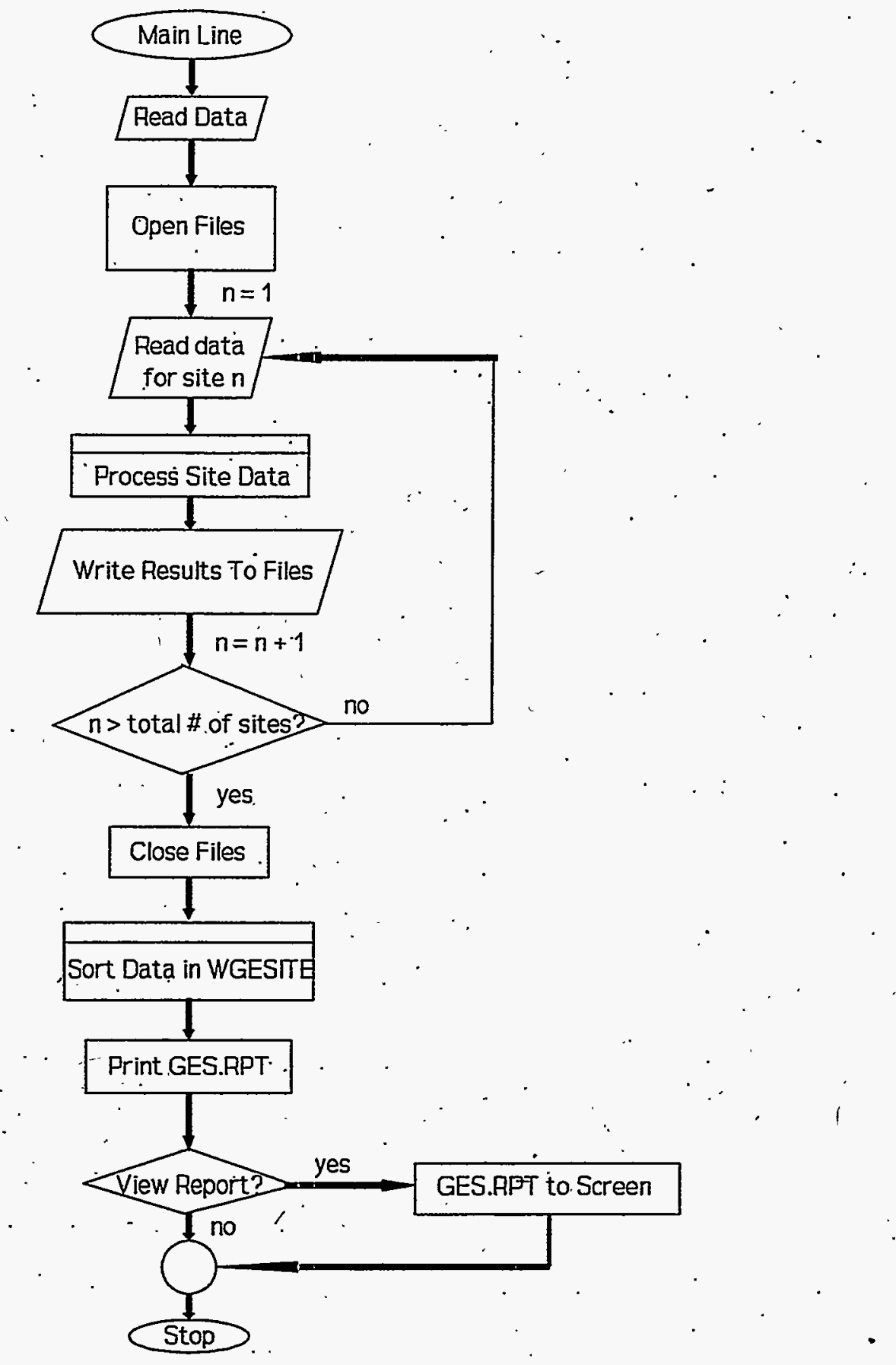


Figure 7E-2. Flow Diagram for Process Site Data Routine

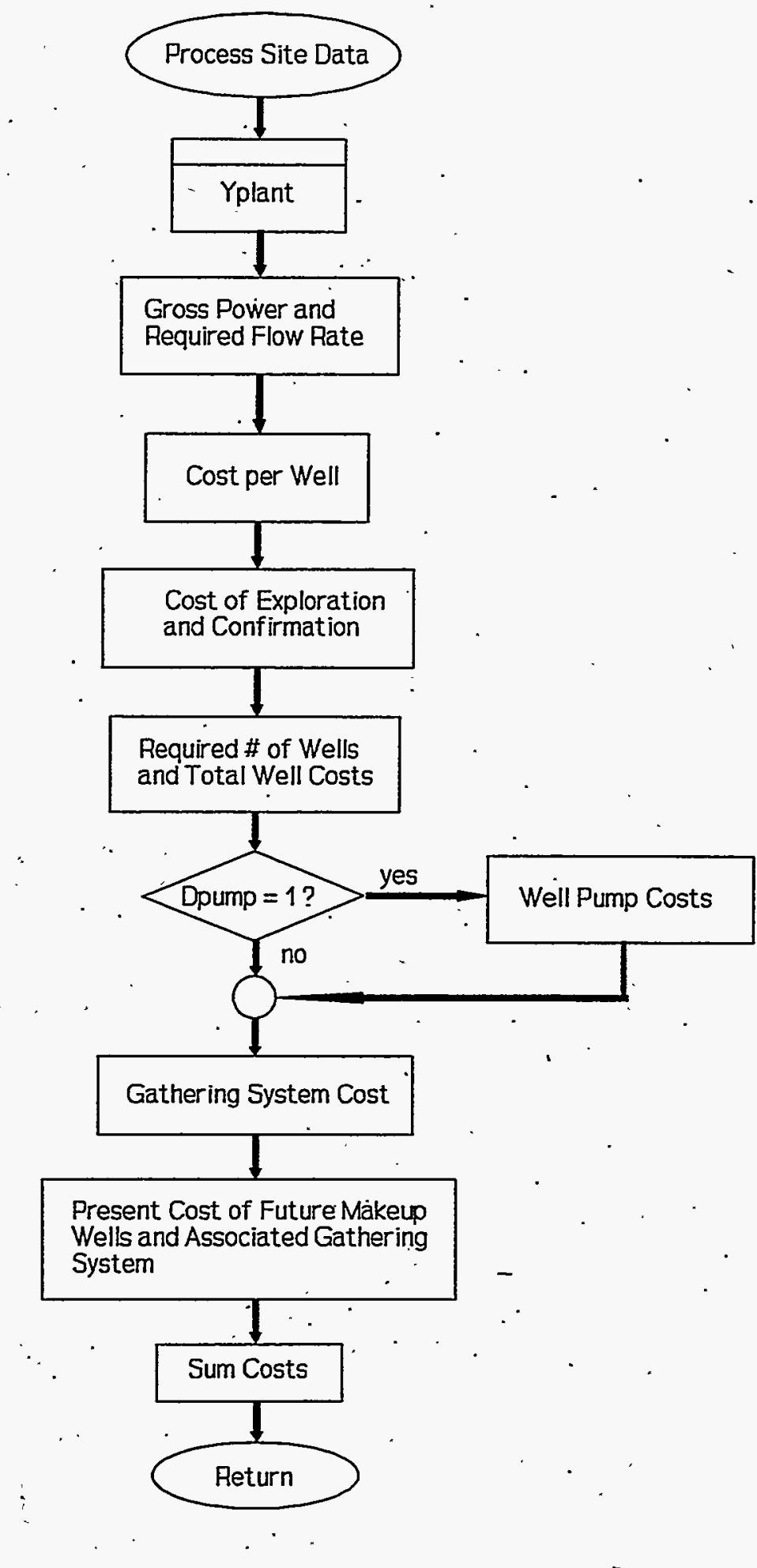


Figure 7E-3. Flow Chart for Yplant Routine

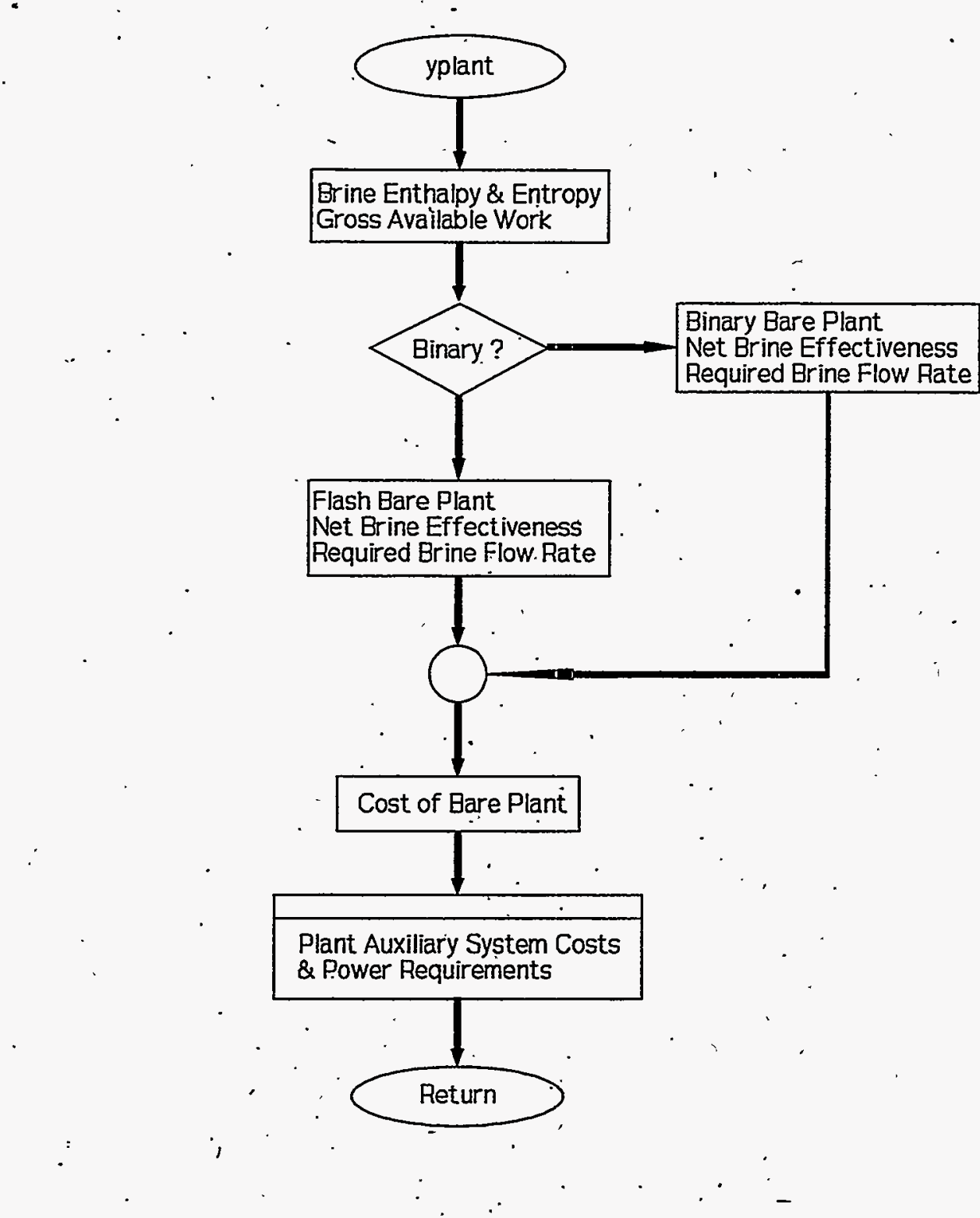




\section{Mathematical Descriptions of Key Algorithms}

\section{Heat Rate (GESDPP)}

$$
\text { HEAT.RATE }(\mathrm{BTU} / \mathrm{kwh})=1000 *(\text { H.IN }- \text { H.COND) } / \text { NETBE }
$$

where:

$$
\begin{aligned}
& \text { H.IN, = enthalpy of fluid entering plant (BTU/lb) } \\
& \text { H.COND = enthalpy of fluid in the condenser (BTU/lb) } \\
& \text { NETBE = net brine effectiveness (watt-hr/lb) }
\end{aligned}
$$

\section{Plant Costs (GESDPP)}

Flash and binary plants do not require all the same auxiliary systems, and differing site characteristics sometimes dictate different auxiliary systems (such as dry cooling systems in an arid region). For this reason, GESDPP calculates the cost of a bare plant (excluding auxiliary systems) and then calculates the additional costs for the necessary auxiliary systems. Unless otherwise noted, capital costs are expressed in 1990 dollars per kilowatt capacity and O\&M costs are in either millions of 1990 dollars per year or millions of 1990 dollars per $\mathrm{kw}$ capacity per year.

\section{Cost of Bare Plant (GESDPP) -}

Based on data fits during development of IM-GEO in 1987 and modifications in 1989 to reflect. more recent cost data, the capital cost $(\$ / \mathrm{kw})$ of a bare (excluding auxiliary systems) flash plant was

$$
\begin{aligned}
\text { COST.FLASH }= & 0.75 *\left(1.3336-5.4308 * T X+0.013179 * T X^{2}+1.0752 *\right. \\
& (E F F I C-30))
\end{aligned}
$$

where:

$$
\begin{aligned}
& T X=\text { RESERVOIR.TEMPERATURE (degrees F) }-300 \\
& \text { EFFIC }=\text { efficiency according to the 2nd law of thermodynamics as } \\
& \text { calculated based on physical properties of the geothermal brine. }
\end{aligned}
$$

The capital cost ( $\$ / \mathrm{kw})$ equation for a bare (excluding auxiliary systems) binary plant was developed in 1987 based on the cost of the $50 \mathrm{MW}$ Heber binary plant in California and modified in 1989 to reflect more recent cost data: 


$$
\begin{aligned}
\text { COST.BINARY }= & 1.06 *\left(1595-4.9836 * \text { TX }+0.0087648 * T^{2}+\right. \\
& 3.4082 *(E F F I C-40))
\end{aligned}
$$

where:

$$
\begin{array}{lll}
\text { TX } & = & \text { RESERVOIRTEMPERATURE }-260 \\
\text { EFFIC } & = & \text { same as above }
\end{array}
$$

\section{Cost of Auxiliary Systems (GESDPP)}

Binary plants require a heat exchanger (HXC) which is included in the bare plant cost. However, the capital cost of the HXC is isolated to determine the operation and maintenance costs associated with the HXC. The capital cost of the HXC is:

$$
\text { COST.HXC }=\dot{H X F} * \text { COST.BINARY }
$$

where:

$$
\begin{aligned}
& \begin{array}{l}
\text { HXF } \\
=\quad \text { the fraction of the bare plant cost attributable to the HXC } \\
0.2-0.1 *(R E S E R V O I R . T E M P E R A T U R E-250) / 150
\end{array} \\
& \text { or, if RESERVOIR.TEMPERATURE }>=400 \text { then } H X F=0.1 \\
& \text { or, if RESERVOIR.TEMPERATURE }<=250 \text { then } H X F=0.2
\end{aligned}
$$

The annual cost ( $\$$ million/ $/ \mathrm{kw} / \mathrm{yr})$ of the operation and maintenance of the HXC is:

$$
\text { HXC.O\&M }=0.030 * \text { COST.HXC }
$$

The capital costs of the brine injection pumps are a function of the power required to run them:

$$
\begin{aligned}
\text { FLASH.INJ.POWER }= & 100.3774-0.7504002 * \text { T.IN }+2.116543^{-3} * T^{-1} N^{2} \\
& -2.638822^{-6} * T^{2} . N^{3}+1.220977^{-9} * T^{\prime} N^{4}
\end{aligned}
$$

FLASH.INJ.PUMP.COST $(\$ / \mathrm{kw})=.230 *$ FLASH.INJ.POWER

$$
\begin{aligned}
\text { BINARY.INJ.POWER }= & 16.89875-5.613919^{-2} * \text { T.IN }-1.016828^{-4} * T^{-I N}{ }^{2} \\
& +5.666449^{-7} * T_{. I N^{3}-5.496635^{-10} * T_{. I N}^{4}}
\end{aligned}
$$

$$
\text { BINARY.INJ.PUMP.COST }(\$ / \mathrm{kW})=230 * \text { BINARY.INJ.POWER }
$$

where:

$$
\text { T.IN . = plant inlet temperature (degrees F) }
$$


The annual operating cost for the bare plant and injection pumps is 2 percent of the capital cost of the bare plant and injection pumps.

The cost of the bare plant includes the cooling system. However, where dry cooling is necessary as indicated by the site data, an extra $\$ 111 / \mathrm{kw}$ is added to the cost of the bare plant. Often brine condensate is used for cooling tower water. Water losses in the cooling system are calculated by:

WATER.LOSS (acre-ft/kw/yr) $=2300 /($ RESERVOIR.TEMPERATURE * 1234) where:

$$
\text { RESERVOIR.TEMPERATURE }=\text { reservoir temperature (degrees } \mathrm{F})
$$

The annual cost of the water is calculated by

$$
\text { TOTAL.WATER.COST }(\$ / \mathrm{kw})=\text { WATER.LOSS } * \text { WATER.COST }
$$

where:

$$
\text { WATER.COST = cost of water }(\$ / \text { acre-ft })(\text { from site data) }
$$

For sites where the brine chemistry includes high concentrations of total dissolved solids, precipitation (scaling) of the solids can be problematic. As a result of being designed to operate at temperatures and pressures to avoid precipitation of dissolve solids, binary plants incur additional capital and O\&M costs. Flash plants incur additional capital and O\&M costs by including a clarifier, an auxiliary system to precipitate and remove the dissolved solids upstream from the turbine. The costs for the flash system are:

$$
\begin{aligned}
& \text { CLARIFIER.COST }(\$ / \mathrm{kw})=140 * B \\
& \text { CLARIFIER.O\&M }(\$ / \mathrm{kw} / \mathrm{yr})=3 * \boldsymbol{B}
\end{aligned}
$$

where:

$$
\begin{array}{rlll}
B & 0 & & \text { if total dissolved solids }<0.5 \text { percent } \\
& = & & \text { if total dissolved solids }>=0.5 \text { percent } \\
& =2 & \text { if total dissolved solids }>=10 \text { percent }
\end{array}
$$

$$
\text { CLARIFIER.WASTE.DISPOSAL (\$/kw/yr) = }
$$

where:

$$
S L U D G E(\text { tons/yr })=57.2769 * \text { TFLIN * TDS * (CAP.FAC + 1) }
$$


where:

$$
\begin{array}{lll}
\text { TFLIN } & = & \text { brine flow into plant (million } \mathrm{lbs} / \mathrm{hr} \text { ) } \\
\text { TDS } & = & \text { percentage total dissolved solids } \\
\text { CAP.FAC } & = & \text { capacity factor (fraction) }
\end{array}
$$

General flesh plant $O \& M$ costs (apart from clarifier O\&M costs) due to scale precipitation vary with the amount of total dissolved solids (TDS) in the brine. At TDS $<=2$ percent, the O\&M cost is zero. At TDS $=15$ percent, the O\&M costs are 0.2 percent of the bare plant cost:

$$
\begin{aligned}
& \text { FLASH.SCALE.O\&M }=0.002 *(\text { TDS/15) } *(\text { COST.FLASH + } \\
& \text { INJ.PUMP.COST) }
\end{aligned}
$$

The additional scale-related costs for a binary plant are 50 percent of the cost. of the heat exchanger at a TDS of 15 percent:

$$
\begin{aligned}
& \text { BINARY.SCALE.COST }=0.50 *(\text { TDS/15) * COST.HXC } \\
& \text { BINARY.SCALE. O\&M }=0.015 *(\text { TDS } / 15) * \text { COST.HXC }
\end{aligned}
$$

Total dissolved solids also effect capital costs by increasing corrosion. These effects are also scaled based on TDS $=15$ percent:

$$
\begin{aligned}
& \text { FLASH.CORROSION.COST }= \begin{array}{l}
0.03 *(\text { TDS/15) } \\
\text { INJ.PUMP.COST })
\end{array} \\
& \text { BINARY.COST.FLASH }+ \\
& .
\end{aligned}
$$

The existence of noncondensible gases (NCG) in the brine have no effect on binary systems, but at concentrations greater than 0.5 percent, NCG content effects the cost of flash systems:

$$
\begin{gathered}
\text { FLASH.NCG.COST }(\$ / \mathrm{kw})=13.5 * N C G \\
\text { FLASH.NCG.O\&M }(\$ / \mathrm{kw} / \mathrm{yr})=0.02 * 13.5 * \mathbf{N C G}
\end{gathered}
$$

where:

$$
\text { NCG . . = per cent noncondensible gas content of brine }
$$

The existence of hydrogen sulphide $\left(\mathrm{H}_{2} \mathrm{~S}\right)$ in the brine has no effects on the cost of a binary system since the brine is contained in a closed system. However, with flash systems, to avoid emitting $\mathrm{H}_{2} \mathrm{~S}$ to the atmosphere, an auxiliary abatement system is necessary at sites where such emissions would otherwise exceed regulatory limits. The costs for the abatement system are:

$$
\begin{aligned}
H_{2} S . C O S T= & \left(3,010,000 *(S A / 2,000,000)^{0.60}+230,000\right) * \\
& \cdot 1.643 /(P L A N T . S I Z E / .1000)
\end{aligned}
$$




$$
H_{i} S . O \& M=1.58 *[67,200+R W F(211.18 B T C+173.44 S R E)] . /
$$

where:

$S A$

$$
=\quad R W F * B T C=\text { steam flow from condenser }(\mathrm{lb} / \mathrm{hr})
$$

where:

$$
\begin{aligned}
& R W F \quad . \quad=\quad \text { brïne flow rate into plant }(\mathrm{lb} / \mathrm{hr}) \\
& \text { BTC = fraction of brine flowing through condenser } \\
& \text { PLANT.SIZE } \quad=\text { net power output (MW) }
\end{aligned}
$$

The plant capital and O\&M costs are then summed:

$$
\text { SCCPOWPL = capital costs of plant }+ \text { heat exchanger }+ \text { cooling tower }+
$$
auxiliaries(scaling, corrosion, clarifier) $+\mathrm{H}_{2} \mathrm{~S}$ abatement system

$$
\begin{aligned}
& \text { SCCPOWPLOM }= \text { O\&M costs of plant }+ \text { heat exchanger }+ \text { cooling tower }+ \\
&\text { auxiliaries (scaling; corrosion, clarifier })+\mathrm{H}_{2} \mathrm{~S} \text { abatement system }
\end{aligned}
$$

\section{Emissions Rates}

The $\mathrm{CO}_{2}$ and $\mathrm{H}_{2} \mathrm{~S}$ emission rates for binary plants are zero.

The $\mathrm{CO}_{2}$ emission rate for a flash plant is:

$$
S C O 2 R A T E=1,000,000 / \text { NETBE } * X 12 * N C G / 100 * 0.855
$$

where:

$$
\begin{array}{lll}
\text { NETBE } & = & \text { net brine effectiveness, watt-hour/lb. brine } \\
X 12 & = & \text { steam total mass fraction to turbine } \\
N C G & \cdots & =
\end{array}
$$

The $\mathrm{H}_{2} \mathrm{~S}$ emission rate for a flash plant is:

If the $\mathrm{H}_{2} \mathrm{~S}$ load is greater than the permissible rate then:

$$
\text { SH2SRATE }=\text { H2S.LIMIT } / 453.6 * \text { V.KWH.RATE / NETBE }
$$


where:

$$
\begin{array}{lll}
\text { H2S.LIMIT. } & = & \text { regulatory limit for } \mathrm{H}_{2} \mathrm{~S} \text { emission, gram/hr/MW (site data) } \\
\text { V.KWH.RATE } & = & \text { work rate across turbine, watt-hour/lb. brine } \\
\text { NETBE } & = & \text { net brine effectiveness, watt-hour/lb. brine }
\end{array}
$$

If the $\mathrm{H}_{2} \mathrm{~S}$ load is less than or equal to the permissible rate then:

$$
\text { SH2SRATE }=\text { H2S.LOAD / } 453.6 * \text { V.KWH.RATE / NETBE }
$$

where:

$\begin{array}{lll}\text { H2S.LOAD } & = & \mathrm{H}_{2} \mathrm{~S} \text { emission load, gram } / \mathrm{hr} / \mathrm{MW} \\ \text { V.KWH.RATE } & = & \text { work rate across turbine, watt-hour/lb. brine } \\ \text { NETBE } & = & \text { net brine effectiveness, watt-hour/lb. brine }\end{array}$

\section{Field Development Costs (GESDPP)}

Geothermal field development consists of three phases - exploration, confirmation, and construction. Various well testing costs are added to the cost per well depending on which phasethe well is drilled in. First, a base well cost is determined for both nominal diameter and slim diameter (used only in exploration phase) wells. Then, the cost of each of the three phases is determined.

\section{Well Costs·(GESDPP)}

The cost per well (sans well testing costs) for nominal diameter wells.is calculated by adding the 3 problem costs (loss circulation, cementing, miscellaneous) to the drilling cost (all of which are site data) and then adding additional costs to reflect that a percentage of wells will need to be either redrilled or extended:

$$
W C P W=\underset{\text { well cost per well }}{\text { BASE. WELL }} \text { COST }+ \text { COST } . E X T N D+C O S T \cdot R E D R I L L=
$$

where:

$$
\begin{array}{llc}
\text { BASE.WELLCOST } & = & \text { drilling cost }+ \text { problems costs (all site data) } \\
\text { COST.EXTND } & = & 0.75 *(0.8-0.25 * \text { WDRY }) * \text { WCEXTEND }
\end{array}
$$


where:

$$
\begin{aligned}
& \text { WDRY. = fraction of wells that are dry (from site data) } \\
& \text { WCEXTEND = cost to extend one well (site data) } \\
& \text { COSF.REDRILL = } 0.165 * \text { WRED * WCBASE }
\end{aligned}
$$

where:

$$
\begin{array}{lll}
W R E D & = & \text { redrilling } \text { incidence fraction (site data) } \\
W C B A S E & = & \text { drilling cost (site data) }
\end{array}
$$

The cost for slim diameter-wells is:

SLIM.W.CPW $=0.5 *$ (drilling cost $+0.6 *$ lost circulation problem costs + miscellaneous problem costs (all from site data))

\begin{tabular}{|c|c|c|}
\hline UCOST1 & $=$ & cost of geological and geophysical investigations \\
\hline N.IDENT. & $=$ & number of wild cat wells required to get one good one \\
\hline$U \operatorname{COST2}$ & $=$ & cost of drilling shallow thermal gradient wells \\
\hline WC.WILD & $=$ & $\begin{array}{l}\text { cost of drilling a slim.diameter well } \\
\text { SLIM.W.CPW + TEST.SL.EXPL }\end{array}$ \\
\hline
\end{tabular}

Exploration Phase Cost (GESDPP)

$$
\begin{aligned}
& \text { C.EXPL.TOTAL }=(\text { UCOST1 }+ \text { N.IDENT * } \\
&\text { WC.WLLD })) / \text { UPROB.CONF }
\end{aligned}
$$

where:

where:

$$
\begin{aligned}
& \text { SLIM.W.CPW = }(\text { see Well Costs above }) \\
& \text { TEST.SL.EXPL }=\text { cost to test a slim well } \\
& \text { UPROB.CONF } \quad=\text { probability of successful confirmation (site data) }
\end{aligned}
$$




\section{'Confirmation Phase Cost (GESDPP)}

The cost of unsuccessful wells during the confirmation period is:

$$
\text { SCCDHCONF }=((G O O D . I N J S+D R Y . C O U N T) * \text { WC.GENL })+\text { C.PERMITS }
$$
where:

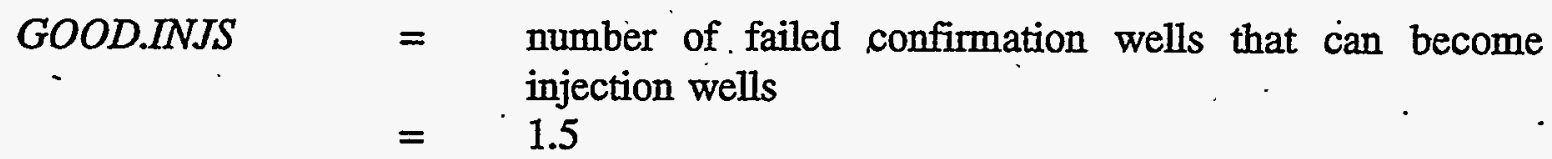

$D R Y . C O U N T=$ number of failed confirmation wells unsuitable for injection wells WC.GENL $=W C P W+$ TEST.U.IJ

where:

$$
\begin{array}{ll}
\text { WCPW } & =\quad \text { (see Drilling Cost above) } \\
\text { TEST.U.IJ } & =\text { cost of logging and 3. day flow test } \\
\text { C.PERMITS } & =
\end{array}
$$

The cost of successful production wells during the confirmation period is:

$$
\text { CONF.PROD }=\text { GOOD.PRODS } * \text { WC.CONF }
$$

where:

$$
\begin{aligned}
& \text { GOOD.PRODS } \quad=\quad \text { number of successful confirmation production wells }=4 \\
& \text { WC.CONF }=\text { cost per successful confirmation well } \\
& =\quad W C P W+\text { TEST.U.CONF }
\end{aligned}
$$

The cost of injection wells, and successful and unsuccessful production wells during the construction phase is: 


$$
\text { CÓST.INJ.WELLS }=\text { WC.GENL } *(\text { WIJN - GOOD.INJS })
$$

COST.PRO.WELLS $=($ WNUM + WPSPR - GOOD.PRODS $) *$

WC.PROD * $(1+W R E D)$

$$
\text { COST.DRY.WELLS }=\text { WC.GENL * WPDRY }
$$

\begin{tabular}{|c|c|c|}
\hline WC.GENL & $=$ & (see Confirmation Cost above) \\
\hline WIJN & $=$ & total number of injection wells needed \\
\hline GOOD.INJS & $=$ & number of injection wells from confirmation phase $=1.5$ \\
\hline WNUM & $=$ & number of production wells \\
\hline WPSPR & $=$ & number of spare production wells \\
\hline GOOD.PRODS & $=$ & number of good producers from confirmation phase $=4$ \\
\hline WC.PROD & $=$ & $W C P W$ (see above) + cost of logging and 10-day flow tests \\
\hline WRED & $=$ & redrilling incidence fraction (from site data) \\
\hline WPDRY & $=$ & number of construction phase dry holes \\
\hline
\end{tabular}

where:

\section{Well Pump Cost (GESDPP)}

The cost of downhole weil pumps is:

$$
D P T L=D P C * P N U M
$$

where:
$D P \dot{C}$
$=\quad$ deep pump cost, $\$$ million
PNUM
$=$ number of pumps (including spares)

\section{Gathering System Cost (GESDPP)}

The cost of the surface gathering system for the production wells assumes a rectangular field, pipe cost of $\$ 50$ per foot, and a $\$ 20,000$ control valve for each well. The cost is: 
CPRODGATH $(\$$ million $)=1.120 *(50 *$ NFEET $+20,000 *$

WPRODSUM) / 1,000,000

where:

NFEET $\quad=$ length of pipe per production well, $\mathrm{ft}$.

WPRODSUM $\quad=\quad$ total number of production wells (including spares)

The cost of the surface gathering system for the injection wells assumes location of the injection wells in groups of 4, with total length of piping for each group equal to 6 times the distance between wells (site data), and a $\$ 20,000$ control valve for each well. The cost is:

CINJGATH (\$ million) $=1.120 *$ COSTPER4 $*$ WIJN $/ 4+0.020 *$ WIJN where:

COSTPER $4: \quad=\quad$ surface pipe cost per group of 4 injection wells, $\$$ million

WIJN $\quad=$ total number of injection wells needed

Field O\&M Cost (GESDPP)

Operation and Maintenance costs (\$ million/yr) associated with production wells and injection wells are:

$$
\begin{aligned}
& \text { WELL.O\&M }= 0.506+\text { WNUM } \\
& \text { V.INJ.REWORK V.PROD.REWORK + WIJN * }
\end{aligned}
$$

\begin{tabular}{|c|c|c|}
\hline WNUM & $=$ & total number of production wells \\
\hline V.PROD.REWORK & $=$ & unit annual cost for production well rework \\
\hline WIJN & $=$ & total number of injection wells \\
\hline V.INJ.REWORK & & unit annual cost of injection wells \\
\hline
\end{tabular}

where:

Operation and maintenance costs ( $\$$ million/yr) for production well pumps are: 


$$
\text { PUMP.O\&M }=0.0224 * \text { WNUM }
$$

where:

WNUM = total number of production wells

Operation and maintenance costs ( $\$$ million/yr) for the surface gathering system are:

$$
\text { GATHER.O\&M }=0.01 * B B B * S P T L
$$

where:

$$
\dot{B B B}=Z Z B^{2}-Z Z B+2
$$

where:

$$
\begin{array}{lll}
Z Z B & \fallingdotseq & 0 \text { if } T D S<10 \\
& = & 1 \text { if } T D S>10 \\
& = & 2 \text { if } T D S>100
\end{array}
$$

where:

TDS . = total dissolve solids (parts per thousand) (site data)

\section{Injection Well Replacement Cost (GESDPP)}

:The proper location of injection wells is difficult. The objective is to locate them so that the injected cooled brine replenishes reservoir volume and pressure without cooling the brine coming up the production wells. It is not uncommon for initial injection wells to have to be relocated due to this "thermal breakthrough" of the cooled injectate into the production stream. The cost of relocating poorly located injection wells is:

$$
\begin{aligned}
\text { COOLED.CAP.COST }= & (\text { WIJN } * \text { WC.GENL }+ \\
& \text { CINJGATH })(P E R C E N T . I N J . F A I L E D / 100)
\end{aligned}
$$

where:

PERCENTIINJ.FAILED = percent of injectors to fail_(site dàtá) 